\title{
Relative set theory: Internal view
}

\author{
KAREL HRBACEK
}

\begin{abstract}
A nonstandard set theory with many levels of standardness was first proposed by Yves Péraire [28]. The theory GRIST formulated here is an extension of Péraire's RIST. We prove that it is conservative and categorical over ZFC, and universal among theories of its kind. Technically, the paper carries out detailed analysis of the construction of internally iterated ultrapowers introduced in the author's [13]. The objectives of an eventual extension of GRIST to a full relative set theory are briefly discussed in the last section.
\end{abstract}

2000 Mathematics Subject Classification 03C20 (primary); 03C62, 03E70, $03 \mathrm{H} 99$ (secondary)

Keywords: ultrafilter, ultraproduct, iterated ultrapower, factoring lemma, RudinFrolík ordering, nonstandard analysis, nonstandard set theory, IST, RIST

\section{Introduction.}

Nonstandard analysis, a modern rigorous theory of infinitesimals, was erected on model-theoretic foundations by Abraham Robinson. Axiomatic treatments of nonstandard analysis in the framework of set theory originated in the author's [11] and Nelson's [27]; Kanovei and Reeken's monograph [22] provides an exhaustive survey and comparison of the currently established nonstandard set theories. A common feature of the model-theoretic approach and (most of) the axiomatic approaches is a fixed classification of the objects of discourse into two or three kinds: standard, internal, and usually also external. In an alternative approach, first proposed by Wallet, Péraire and Gordon, infinitesimals and other "nonstandard" concepts are relative to a given, but arbitrary, level of standardness, metaphorically interpretable as a "level of knowledge." Péraire [28] developed an axiomatic theory RIST (Relative Internal Set Theory) that formalizes this outlook, and proved that it is a conservative extension of ZFC. (See [10] for Gordon's different approach.) The formulations of the axioms of RIST are somewhat cumbersome, due to the fact that RIST does not admit quantification over levels of standardness. The author's theory FRIST [13] removes this limitation; as a result, it is both more powerful and formally simpler than RIST. 
Theories with many levels provide the users of nonstandard methods with new tools (see for example Péraire [30]) whose power needs to be further explored, but—somewhat paradoxically - their main advantage may be in exposition. Nonstandard analysis raised the hope that the familiar $\epsilon-\delta$ definitions of the fundamental calculus conceptsderivative, limit, integral — could be replaced by more intuitive ones using infinitesimals in the style of Leibniz. In traditional nonstandard analysis one can do that only for standard functions at standard points. For internal points and/or functions, definitions of these concepts have to fall back on the $\epsilon-\delta$ method, as there are no infinitesimals relative to the internal level. For elementary expositions it is advantageous to adopt the "internal picture" of Nelson, in which internal sets are identified with the "usual" ones; this picture avoids the need to discuss nonarchimedean "hyperreals", but the problem of avoiding $\epsilon-\delta$ for all internal functions and points then becomes particularly acute. Theories with many levels solve this problem in a simple, natural way. Some of these issues are discussed in detail in [16].

In a joint paper with O'Donovan and Lessmann [17] and a book manuscript in preparation [18], we attempt to demonstrate that elementary analysis at a beginner's level can be developed from a few very simple axioms that form a small fragment of FRIST (but transcend RIST in some important aspects). Several high school level calculus courses in Geneva have been successfully taught with this approach in Spring 2009.

This paper is concerned with metamathematics of internal relative set theories. The experience with writing [18] showed that principles beyond those of FRIST are useful for some more advanced arguments in analysis. Here we present an extension of FRIST denoted GRIST, and prove that GRIST is a conservative extension of ZFC. Moreover, GRIST is complete over ZFC in a technical sense (Corollary 12.7) and universal among all theories of relative standardness satisfying some minimal axioms (Corollary 12.6). The interpretation of GRIST in ZFC that we use is the same as the one given in Section 6 of [13] for a version of FRIST. It is quite complicated, and the proof that GRIST is valid in it even more so. In contrast, the axioms of GRIST are fairly simple (see below). For these reasons, and unlike the usual practice in traditional nonstandard analysis, use of GRIST as a foundation for development of mathematics has to be based on its axioms rather than its models.

The language of GRIST has two primitive binary predicate symbols, $\epsilon$ and $\sqsubseteq$. We read $x \sqsubseteq y$ as " $x$ is $y$-standard" or " $x$ appears at the level of $y$ ".

We define $x \sqsubseteq \alpha y$ by $x \sqsubseteq \alpha \vee x \sqsubseteq y$. If $\mathcal{P}\left(x_{1}, \ldots, x_{k}\right)$ is a formula in the $\in$ - $\sqsubseteq$ language and $\alpha$ a variable, $\mathcal{P}^{\alpha}\left(x_{1}, \ldots, x_{k}\right)$ is obtained from $\mathcal{P}\left(x_{1}, \ldots, x_{k}\right)$ by replacing every occurrence of $\sqsubseteq$ with $\sqsubseteq \alpha$. 


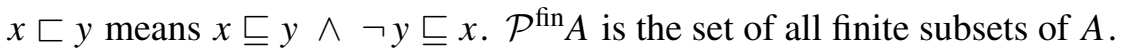

We postulate all axioms of ZFC (with Separation and Replacement for $\in$-formulas). The remaining axioms are:

\section{Relativization}

$\sqsubseteq$ is a dense total pre-ordering with a least element 0 and no greatest element.

In detail, the conjunction of:

$$
\begin{array}{ll}
(\forall x)(0 \sqsubseteq x \wedge x \sqsubseteq x) ; \quad(\forall x, y, z)((y \sqsubseteq x \wedge z \sqsubseteq y) \Rightarrow z \sqsubseteq x) ; \\
(\forall x, y)(x \sqsubseteq y \vee y \sqsubseteq x) ; \quad(\forall x)(\exists y)(x \sqsubset y) ; \quad(\forall x, y)(x \sqsubset y \Rightarrow(\exists z)(x \sqsubset z \sqsubset y)) .
\end{array}
$$

\section{Transfer}

If $\alpha \sqsubseteq \beta$, then, for all $x_{1}, \ldots, x_{k} \sqsubseteq \alpha$,

$$
\mathcal{P}^{\alpha}\left(x_{1}, \ldots, x_{k}\right) \Leftrightarrow \mathcal{P}^{\beta}\left(x_{1}, \ldots, x_{k}\right) .
$$

\section{Standardization}

For any $\alpha \sqsupset 0$ and any $A, x_{1}, \ldots, x_{k}$, there exists $\beta \sqsubset \alpha$ and $B \sqsubseteq \beta$ such that, for every $\gamma$ with $\beta \sqsubseteq \gamma \sqsubset \alpha$,

$$
(\forall y \sqsubseteq \gamma)\left(y \in B \Leftrightarrow y \in A \wedge \mathcal{P}^{\gamma}\left(y, A, x_{1}, \ldots, x_{k}\right)\right) .
$$

\section{Idealization}

For all $A \sqsubset \beta$ and all $x_{1}, \ldots, x_{k}$,

$$
\begin{array}{r}
\left(\forall a \in \mathcal{P}^{\text {fin }} A\right)\left[a \sqsubset \beta \Rightarrow(\exists y)(\forall x \in a) \mathcal{P}^{\beta}\left(x, y, A, x_{1}, \ldots, x_{k}\right)\right] \\
\Leftrightarrow(\exists y)(\forall x \in A)\left[x \sqsubset \beta \Rightarrow \mathcal{P}^{\beta}\left(x, y, A, x_{1}, \ldots, x_{k}\right)\right] .
\end{array}
$$

\section{Granularity}

For any $x_{1}, \ldots, x_{k}$, if $(\exists \alpha) \mathcal{P}^{\alpha}\left(x_{1}, \ldots, x_{k}\right)$, then

$$
(\exists \alpha)\left[\mathcal{P}^{\alpha}\left(x_{1}, \ldots, x_{k}\right) \wedge(\forall \beta)\left(\beta \sqsubset \alpha \Rightarrow \neg \mathcal{P}^{\beta}\left(x_{1}, \ldots, x_{k}\right)\right)\right] .
$$

An outline of the contents of the paper follows.

Our key objective is to show that every model $\mathbf{M}$ of $\mathbf{Z F C}$ can be extended to a model of GRIST. It is also crucial that the extension be definable in $\mathbf{M}$. To accomplish both of 
these purposes, it is more convenient to work with interpretations of GRIST (and other extensions of $\mathbf{Z F C}$ ) in $\mathbf{Z F C}$, rather than directly with models. Section 1 summarizes some facts about interpretations, and in particular focuses on those where the universe of standard sets in the interpretation is isomorphic to the universe of $\mathbf{Z F C}$; inspired by the terminology of Kanovei and Reeken [21], we call them realizations. Section 1 also establishes notational conventions for the rest of the paper. The material in this section can be consulted for reference only, if/when needed.

Sections $2-5$ deal with (internal) nonstandard set theories, that is, extensions of ZFC that have two kinds of objects, standard and internal. We show that limit ultrapowers of the universe $\mathbb{V}$ of set theory are precisely the realizations of a basic nonstandard set theory ST. We then consider an extension of ST by the Back and Forth Property; the resulting theory is equivalent to BST of Kanovei [20]. Here we prove that BST is distinguished among nonstandard set theories by being categorical over ZFC in the sense that for every countable model $\mathbf{M}$ of $\mathbf{Z F C}$ there is a unique countable model of BST with $\mathbf{M}$ as its standard universe. BST is also universal in the sense that every countable model of ST embeds into any model of BST with the same standard universe. Most of this material is known in some form. In particular, we rely heavily on Gordon's notion of relative standardness captured by $\mathbb{S}[a]$, Gordon and Andreev's use of monads [2], Kanovei's technique for defining an interpretation of BST in ZFC [22], and ideas explicitly and implicitly contained in Andreev and the author's [3]. We give here a unified presentation in a form suitable for generalization to theories with many levels of standardness. This generalization is worked out in Sections $6-11$.

In Section 6 we define trees of ultrafilters, a framework for transfinite repetition of the ultrapower construction (stratified ultrapowers) investigated in Section 7. Section 8 introduces a basic theory with many levels of standardness, the stratified set theory SST. All stratified ultrapowers satisfy axioms of a stronger theory SST* . The technical heart of the paper is in Sections 9 and 10. We define stratified ultrafilters (= canonical trees of ultrafilters) and show, in SST $^{*}$, that for every set $x$ there is a standard stratified ultrafilter $U$ such that $x \mathbf{M} U$, where $x \mathbf{M} U$ is a generalization to many-leveled context of the notion " $x$ is in the monad of $U$ ". It is defined via the key concept of pedigree. We then formulate the theory SST ${ }^{\sharp}=$ SST + Back and Forth Property. In Section 11 we prove that the interpretation for FRIST from Section 6 of [13] satisfies SST ${ }^{\sharp}$.

The axioms of SST $\mathbf{T}^{\sharp}$ are conceptually elegant, but quite unsuitable for development of mathematics. In Section 12 we show that SST $\sharp$ is equivalent to GRIST. It then follows that GRIST is distinguished among stratified set theories by properties analogous to those that distinguish BST among nonstandard set theories. Section 12 concludes with a number of consequences of GRIST, important because they have either already 
been found useful for mathematical applications of GRIST, or because they generalize similar results for BST or RIST.

This paper is concerned only with internal sets, although a fully adequate theory of nonstandard objects undoubtedly has to account for external sets as well; see the author's [11, 14]. The concluding section contains some remarks on external sets and GRIST; however, a detailed study of this topic raises a number of new issues that will be addressed elsewhere.

Acknowledgements. This work was supported by the Scholar Incentive award from City College of New York in the Fall 2005, and by the PSC-CUNY grant 67486-00 36 in 2005 - 2006. I have reported on it at the congresses "Nonstandard Methods and Applications in Mathematics", Pisa, May 25 - 31, 2006, and "Applications of Ultrafilters and Ultraproducts in Mathematics", Pisa, June 1 - 7, 2008.

I am grateful to Petr Andreev, David Ballard, Mauro Di Nasso, Evgeni Gordon, Vladimir Kanovei, Olivier Lessmann, Richard O'Donovan, Michael Reeken and Yves Péraire for helpful discussions related to the subject of this paper. 


\section{Contents.}

\section{Introduction.}

1 Realizations.

2 Ultrafilters and Ultrapowers.

3 Nonstandard Set Theory.

4 Repeated Ultrapowers.

5 Limit Ultrapowers and BST.

6 Trees of Ultrafilters.

7 Stratified Ultrapowers.

8 Elementary Relative Set Theory.

9 Stratified Ultrafilters.

10 Pedigrees.

11 Stratified Limit Ultrapowers.

11.1 Stratified limit ultrapowers.

11.2 Internally iterated ultrapowers.

11.3 An interpretation for $\mathbf{S S T}^{\sharp}$.

12 GRIST.

12.1 Metamathematics of $\mathbf{S S T}^{\sharp}$.

12.2 Equivalence of GRIST and SST ${ }^{\sharp}$.

12.3 Consequences of GRIST.

12.4 Variations of GRIST.

Conclusion: GRIST and External Sets.

References. 


\section{Realizations.}

We work in the Zermelo-Fraenkel set theory $\mathbf{Z F C}$ unless explicitly stated otherwise. In particular, we often employ the following easy consequence of its axioms.

Selection: Let $\mathcal{P}(x, y, \bar{p})$ be a formula.

$(\forall \bar{p})(\forall A)[(\forall x \in A)(\exists y) \mathcal{P}(x, y, \bar{p}) \Rightarrow(\exists f)(f$ is a function $\wedge(\forall x \in A) \mathcal{P}(x, f(x), \bar{p}))]$.

Here and elsewhere we use overbar as shorthand for a finite list; thus $\bar{p}$ stands for $p_{1}, \ldots, p_{k}$. Jech's monograph [19] is a reference for all undefined set-theoretic concepts and all unproved results about them.

We use classes informally, as a way to speak about extensions of formulas, and usually denote them by letters in blackboard, fractur, or uppercase Greek type. For example, if $\mathcal{P}(x, \bar{p})$ is a formula (in the $\in$-language) and $p_{1}, \ldots, p_{k}$ are sets, then $\mathbb{C}=\{x: \mathcal{P}(x, \bar{p})\}$ is a class. Occasionally, more complicated objects such as systems of classes are convenient; they are interpretable as classes in standard ways. For example, if $\mathbb{A}$ is a class, $(\mathbb{A})_{i}$ denotes the class $\{x:\langle i, x\rangle \in \mathbb{A}\}$, and the system of classes $\left\langle(\mathbb{A})_{i}: i \in I\right\rangle$ is to be interpreted as $\{\langle i, x\rangle \in \mathbb{A}: i \in I\}$.

The principal objects of our study are "structures" (usually, proper classes) for a firstorder language containing equality, the binary predicate symbol $\in$, usually a unary predicate symbol st, and possibly some other predicate symbols. Technically, such objects are called interpretations.

An interpretation for the language $\{\in, \mathbf{s t}, \ldots\}$ is a list of classes (ie, formulas) $\mathfrak{M}:=\left(\mathbb{M},==_{\mathfrak{M}}, \in_{\mathfrak{M}}, \mathbb{S}_{\mathfrak{M}}, \ldots\right)$, where $=_{\mathfrak{M}}$ and $\in_{\mathfrak{M}}$ are subclasses of $\mathbb{M} \times \mathbb{M}, \mathbb{S}_{\mathfrak{M}}$ is a subclass of $\mathbb{M}, \ldots$ If $\mathcal{P}(\bar{x})$ is a formula of the $\{\in, \mathbf{s t}, \ldots\}$-language, $\mathcal{P}^{\mathfrak{M}}(\bar{x})$ is the formula obtained from $\mathcal{P}$ by replacing each occurrence of $x=y, x \in y, \mathbf{s t}(x), \ldots$, resp., by (the formula defining) $x=\mathfrak{M} y, x \in_{\mathfrak{M}} y, \mathbb{S}_{\mathfrak{M}}, \ldots$, resp., and each occurrence of the quantifier $\exists x$ ( $\forall x$, resp.) by $\exists x \in \mathbb{M}$ ( $\forall x \in \mathbb{M}$, resp.). [It may be necessary to suitably rename some variables when doing the replacement. $\mathcal{P}^{\mathfrak{M}}(\bar{x})$ may have free variables other than $\bar{x}$ : the parameters of the formulas defining the components of $\mathfrak{M}$.] See Shoenfield [33] and Kanovei-Reeken [22] for background material on interpretations.

For $\bar{x} \in \mathbb{M}, \mathcal{P}^{\mathfrak{M}}(\bar{x})$ asserts that $\mathcal{P}(\bar{x})$ holds in $\mathfrak{M}$ (or, $\mathfrak{M}$ satisfies $\mathcal{P}(\bar{x})$ ), and we use the notation $\mathfrak{M} \vDash \mathcal{P}(\bar{x})$ for the formula $\mathcal{P}^{\mathfrak{M}}(\bar{x})$, when more convenient.

It is the mathematical practice to extend the language by new predicate, function and constant symbols (defined in terms of $\in, \mathbf{s t}, \ldots$ and previously defined symbols), and we want to apply the notations $\mathcal{P}^{\mathfrak{M}}(\bar{x})$ and $\mathfrak{M} \vDash \mathcal{P}(\bar{x})$ also to formulas of the 
extended language. It is to be understood that all defined symbols in $\mathcal{P}$ are replaced by their definitions before the construction of the formula $\mathcal{P}^{\mathfrak{M}}$. A notational ambiguity threatens when writing expressions like $\mathfrak{M} \vDash \mathcal{P}(\tau(x))$ where $\tau$ is a term. As written, it should be interpreted as $\mathfrak{M} \vDash \mathcal{Q}(x)$, where $\mathcal{Q}(x)$ is the formula obtained from $\mathcal{P}(\tau(x))$ by eliminating all instances of $\tau(x)$ using its definition. However, we also often want to state that $\mathfrak{M} \vDash \mathcal{P}(y)$ where $y=\tau(x)$, that is, the value of the term $\tau(x)$ in the ambient set theory, not in $\mathfrak{M}$, should be substituted for $y$ in $\mathfrak{M} \vDash \mathcal{P}(y)$. We underline the terms that are to be interpreted in the latter sense, that is, evaluated before being substituted into $\mathcal{P}$. For example, $\mathfrak{M} \vDash y=\bigcup x$ means $y={ }_{\mathfrak{M}} \bigcup^{\mathfrak{M}} x$, ie, $(\forall z \in \mathbb{M})\left(z \in \mathfrak{M} y \Leftrightarrow(\exists u \in \mathbb{M})\left(z \in_{\mathfrak{M}} u \wedge u \in_{\mathfrak{M}} x\right)\right)$. In contrast, $\mathfrak{M} \vDash y=\underline{\bigcup x}$ means $y=\mathfrak{M} \bigcup x$. To minimize the need for such underlining, we posit that terms whose leftmost symbol denotes an embedding of interpretations are always evaluated before substituting into $\mathcal{P}$; these symbols include $\mathbb{F}, \mathbb{G}, \varphi^{*}, \psi^{*}, \tau^{*}, \Phi, \Psi, \Omega, \mathfrak{j}, \mathfrak{k}$. The same applies to terms involving the symbol / defined in Section 7. A few exceptions to these conventions occur for the symbols $\mathfrak{k}$ and $*$. They should be obvious from context, and are usually explicitly pointed out.

We require of an interpretation that $=\mathfrak{M}$ is a congruence relation:

$\mathfrak{M} \vDash(\forall x)(x=x)$

$\mathfrak{M} \vDash(\forall x, y)(x=y \Rightarrow y=x)$

$\mathfrak{M} \vDash(\forall x, y, z)(x=y \wedge y=z \Rightarrow x=z)$

$\mathfrak{M} \vDash\left(\forall x, y, x^{\prime}, y^{\prime}\right)\left(x=x^{\prime} \wedge y=y^{\prime} \wedge x \in y \Rightarrow x^{\prime} \in y^{\prime}\right)$

$\mathfrak{M} \vDash\left(\forall x, x^{\prime}\right)\left(\left(x=x^{\prime} \wedge \mathbf{s t}(x)\right) \Rightarrow \mathbf{s t}\left(x^{\prime}\right)\right)$

$\ldots$

For example, the first formula is $(\forall x \in \mathbb{M})(x=\mathfrak{M} x)$ when spelled out in detail.

The equivalence classes modulo $=_{\mathfrak{M}},[x]_{\mathfrak{M}}:=\left\{x^{\prime} \in \mathbb{M}: x^{\prime}=_{\mathfrak{M}} x\right\}$, are in general proper classes. ZFC does not imply existence of a class of representatives for arbitrary partitions into classes, and we prefer to avoid "Scott's Trick" of taking the subset of $[x]_{\mathfrak{M}}$ consisting of $x^{\prime}$ of the least rank, and work with the congruences $=_{\mathfrak{M}}$ rather than the true identity. ${ }^{1}$ This necessitates some care with the definitions of embedding and isomorphism of interpretations.

Let $\mathfrak{M}_{1}, \mathfrak{M}_{2}$ be interpretations, with the language of $\mathfrak{M}_{1}$ included in the language of $\mathfrak{M}_{2}$. An embedding of $\mathfrak{M}_{1}$ to $\mathfrak{M}_{2}$ is a subclass $\mathbb{F}$ of $\mathbb{M}_{1} \times \mathbb{M}_{2}$ such that

\footnotetext{
${ }^{1}$ One reason for this decision is to avoid an additional layer of notation, such as having to write $x_{\mathfrak{M}}$ in place of $x$. More fundamentally, almost all results of this paper remain valid in ZFC with Regularity removed and Selection added; in this theory, Scott's Trick is not available. This observation is important for planned future work on extending Relative Set Theory to external sets (see Conclusion).
} 
(i) $\left(\forall x_{1} \in \mathbb{M}_{1}\right)\left(\exists x_{2} \in \mathbb{M}_{2}\right)\left(\left\langle x_{1}, x_{2}\right\rangle \in \mathbb{F}\right)$

(ii) $\left(\forall x_{1}, y_{1} \in \mathbb{M}_{1}\right)\left(\forall x_{2}, y_{2} \in \mathbb{M}_{2}\right)\left(\left(x_{1}=\mathfrak{M}_{1} y_{1} \wedge x_{2}=\mathfrak{M}_{2} y_{2}\right) \Rightarrow\left(\left\langle x_{1}, x_{2}\right\rangle \in \mathbb{F} \Leftrightarrow\right.\right.$ $\left.\left.\left\langle y_{1}, y_{2}\right\rangle \in \mathbb{F}\right)\right)$

(iii) $\left(\forall x_{1}, y_{1} \in \mathbb{M}_{1}\right)\left(\forall x_{2}, y_{2} \in \mathbb{M}_{2}\right)\left(\left(\left\langle x_{1}, x_{2}\right\rangle \in \mathbb{F} \wedge\left\langle y_{1}, y_{2}\right\rangle \in \mathbb{F}\right) \Rightarrow\left(x_{1}=\mathfrak{M}_{1} y_{1} \Leftrightarrow\right.\right.$ $\left.\left.x_{2}=\mathfrak{M}_{2} y_{2}\right)\right)$

(iv) $\left(\forall x_{1}, y_{1} \in \mathbb{M}_{1}\right)\left(\forall x_{2}, y_{2} \in \mathbb{M}_{2}\right)\left(\left(\left\langle x_{1}, x_{2}\right\rangle \in \mathbb{F} \wedge\left\langle y_{1}, y_{2}\right\rangle \in \mathbb{F}\right) \Rightarrow\left(x_{1} \in \mathfrak{M}_{1} y_{1} \Leftrightarrow\right.\right.$ $\left.\left.x_{2} \in \mathfrak{M}_{2} y_{2}\right)\right)$

(v) $\left(\forall x_{1} \in \mathbb{M}_{1}\right)\left(\forall x_{2} \in \mathbb{M}_{2}\right)\left(\left\langle x_{1}, x_{2}\right\rangle \in \mathbb{F} \Rightarrow\left(\mathbb{S}_{\mathfrak{M}_{1}}\left(x_{1}\right) \Leftrightarrow \mathbb{S}_{\mathfrak{M}_{2}}\left(x_{2}\right)\right)\right)$ $\cdots$

An embedding is an isomorphism iff $\mathfrak{M}_{1}$ and $\mathfrak{M}_{2}$ are interpretations for the same language and, in addition,

(vi) $\left(\forall x_{2} \in \mathbb{M}_{2}\right)\left(\exists x_{1} \in \mathbb{M}_{1}\right)\left(\left\langle x_{1}, x_{2}\right\rangle \in \mathbb{F}\right)$.

It is easy to check that $=\mathfrak{M}$ is an isomorphism of $\mathfrak{M}$ and $\mathfrak{M}$, that $\mathbb{F}_{2} \circ \mathbb{F}_{1}$ is an embedding of $\mathfrak{M}_{1}$ to $\mathfrak{M}_{3}$ if $\mathbb{F}_{1}$ embeds $\mathfrak{M}_{1}$ to $\mathfrak{M}_{2}$ and $\mathbb{F}_{2}$ embeds $\mathfrak{M}_{2}$ to $\mathfrak{M}_{3}$, and that $\mathbb{F}^{-1}$ is an isomorphism of $\mathfrak{M}_{2}$ and $\mathfrak{M}_{1}$ if $\mathbb{F}$ is an isomorphism of $\mathfrak{M}_{1}$ and $\mathfrak{M}_{2}$.

Embeddings, as we defined them, are relations. It is easier, and often natural, to work with functions that "generate" embeddings.

Let $\mathbb{G}$ be a (class) function with $\operatorname{dom} \mathbb{G} \subseteq \mathbb{M}_{1}$, ran $\mathbb{G} \subseteq \mathbb{M}_{2}$, and such that

(a) $\left(\forall y \in \mathbb{M}_{1}\right)(\exists x \in \operatorname{dom} \mathbb{G})\left(x=\mathfrak{M}_{1} y\right)$

(b) $(\forall x, y \in \operatorname{dom} \mathbb{G})\left(x=\mathfrak{M}_{1} y \Leftrightarrow \mathbb{G}(x)=\mathfrak{M}_{2} \mathbb{G}(y)\right)$

(c) $(\forall x, y \in \operatorname{dom} \mathbb{G})\left(x \in \mathfrak{M}_{1} y \Leftrightarrow \mathbb{G}(x) \in \mathfrak{M}_{2} \mathbb{G}(y)\right)$

(d) $(\forall x \in \operatorname{dom} \mathbb{G})\left(\mathbb{S}_{\mathfrak{M}_{1}}(x) \Leftrightarrow \mathbb{S}_{\mathfrak{M}_{2}}(\mathbb{G}(x))\right)$.

Define $\mathbb{F} \subseteq \mathbb{M}_{1} \times \mathbb{M}_{2}$ by $\left\langle x_{1}, x_{2}\right\rangle \in \mathbb{F} \Leftrightarrow(\exists x \in \operatorname{dom} \mathbb{G})\left(x_{1}=\mathfrak{M}_{1} x \wedge x_{2}==_{\mathfrak{M}_{2}} \mathbb{G}(x)\right)$. It is easy to verify that, if $\mathbb{G}$ has properties (a)-(d), then $\mathbb{F}$ is an embedding of $\mathfrak{M}_{1}$ to $\mathfrak{M}_{2} ;$ moreover, if also

(e) $\left(\forall y \in \mathbb{M}_{2}\right)(\exists x \in \operatorname{dom} \mathbb{G})\left(y=\mathfrak{M}_{2} \mathbb{G}(x)\right)$,

then $\mathbb{F}$ is an isomorphism.

We often say that a function $\mathbb{G}$ is an embedding when meaning that the corresponding $\mathbb{F}$ is an embedding. Also, $\mathbb{G}_{1}=\mathbb{G}_{2}$ then stands for $\mathbb{F}_{1}=\mathbb{F}_{2}, \mathbb{G}_{3}=\mathbb{G}_{2} \circ \mathbb{G}_{1}$ means that $\mathbb{F}_{3}=\mathbb{F}_{2} \circ \mathbb{F}_{1}, \mathbb{G}[\mathbb{A}]$ is $\mathbb{F}[\mathbb{A}]$, and so on.

Most interpretations we work with are for a language that includes a (primitive or defined) unary predicate symbol st, interpreted by a class isomorphic to $\mathbb{V}$, the universe of ZFC. Following Kanovei-Reeken [21], a realistic interpretation, or realization for short, is an interpretation $\mathfrak{M}=\left(\mathbb{M},=_{\mathfrak{M}}, \in_{\mathfrak{M}}, \mathbb{S}_{\mathfrak{M}}, \ldots\right)$ and an isomorphism $\mathfrak{k}$ of $\mathcal{V}:=(\mathbb{V},=, \in)$ and $\mathcal{S}_{\mathfrak{M}}:=\left(\mathbb{S}_{\mathfrak{M}},=\mathfrak{M} \cap\left(\mathbb{S}_{\mathfrak{M}} \times \mathbb{S}_{\mathfrak{M}}\right), \in_{\mathfrak{M}} \cap\left(\mathbb{S}_{\mathfrak{M}} \times \mathbb{S}_{\mathfrak{M}}\right)\right)$. [In this and 
similar situations, we often write $\left(\mathbb{S}_{\mathfrak{M}},=\mathfrak{M}, \in_{\mathfrak{M}}\right)$, or even simply $\mathbb{S}_{\mathfrak{M}}$, for $\mathcal{S}_{\mathfrak{M}}$; it being understood that the relations are those of $\mathfrak{M}$, restricted to $\mathbb{S}_{\mathfrak{M}}$.]

Proposition 1.1 The isomorphism $\mathfrak{k}$ in a realization is uniquely determined.

Proof Assume that $\left(\mathfrak{M}, \mathfrak{k}_{1}\right)$ and $\left(\mathfrak{M}, \mathfrak{k}_{2}\right)$ are realizations. Then $\mathbb{F}:=\mathfrak{k}_{2}^{-1} \circ \mathfrak{k}_{1}$ is an automorphism of $\mathcal{V}$. We note that $=\mathcal{V}$ is the true equality, and hence $\mathbb{F}$ is a function. By induction on rank it follows immediately that $\mathbb{F}$ is the identity on $\mathbb{V}$ : if $\mathbb{F}(y)=y$ for all $y$ of rank less than the rank of $x$, then $\mathbb{F}(x)=\{\mathbb{F}(y): y \in x\}=\{y: y \in x\}=x$. Consequently, $\mathfrak{k}_{1}(x)=\mathfrak{M}_{2} \mathfrak{k}_{2}(x)$ for all $x \in \mathbb{V}$.

We say that an interpretation $\mathfrak{M}:=\left(\mathbb{M},=\mathfrak{M}, \in_{\mathfrak{M}}, \mathbb{S}_{\mathfrak{M}}, \ldots\right)$ is bounded iff

$$
(\forall x \in \mathbb{M})\left(\exists a \in \mathbb{S}_{\mathfrak{M}}\right)(x \in \mathfrak{M} a) .
$$

We say that $\mathfrak{M}$ satisfies Transfer iff, for any $\in$-formula $\mathcal{P}\left(x_{1}, \ldots, x_{k}\right)$,

$$
\left(\forall x_{1}, \ldots, x_{k} \in \mathbb{S}_{\mathfrak{M}}\right)\left(\mathfrak{M} \vDash \mathcal{P}\left(x_{1}, \ldots, x_{k}\right) \Leftrightarrow \mathcal{S}_{\mathfrak{M}} \vDash \mathcal{P}\left(x_{1}, \ldots, x_{k}\right)\right) .
$$

The latter definition involves quantification over formulas, but it turns out that, for bounded realizations, validity of Transfer for a single formula suffices. This is an idea going back to Robinson and Zakon [31]. Another proof of finite axiomatizability of Transfer can be obtained by adapting Theorem 2, Theorem 1 and Remark 2 in Part II of Gaifman [9].

Let $\mathcal{F}_{i}(i=1, \ldots, 10)$ be the Gödel operations (see Jech [19]). Each of these operations has a natural defining restricted $\in$-formula $\mathcal{P}_{i}(x, y, z)$ such that (in $\mathbf{Z F C}$, or much less) one can prove $(\forall x, y)(\exists ! z) \mathcal{P}_{i}(x, y, z)$, and $\mathcal{F}_{i}$ is defined by postulating $(\forall x, y) \mathcal{P}_{i}\left(x, y, \mathcal{F}_{i}(x, y)\right)$.

Proposition 1.2 If $\mathfrak{M}$ is a bounded realization,

$\mathfrak{M} \vDash(\forall x, y)(\exists ! z) \mathcal{P}_{i}(x, y, z)$ and

$\left(\forall x, y, z \in \mathbb{S}_{\mathfrak{M}}\right)\left(\mathfrak{M} \vDash \mathcal{P}_{i}(x, y, z) \Leftrightarrow \mathcal{S}_{\mathfrak{M}} \vDash \mathcal{P}_{i}(x, y, z)\right)(i=1, \ldots, 10)$,

then $\mathfrak{M}$ satisfies Transfer. In particular, $\mathfrak{M}$ satisfies ZFC.

Proof We first note that also $\mathcal{S}_{\mathfrak{M}} \vDash(\forall x, y)(\exists ! z) \mathcal{P}_{i}(x, y, z)$, because $\mathcal{S}_{\mathfrak{M}}$ is isomorphic to $\mathcal{V}$. The assumptions say that, for $i=1, \ldots, 10$, and all $x, y, z \in \mathbb{S}_{\mathfrak{M}}$, $\mathcal{S}_{\mathfrak{M}} \vDash z=\mathcal{F}_{i}(x, y) \Leftrightarrow \mathfrak{M} \vDash z=\mathcal{F}_{i}(x, y)$. Following the steps of the proof of Theorem 30 in Jech [19], one shows that for every restricted formula $\mathcal{P}(\bar{x})$ there is a composition of Gödel operations $\mathcal{F}(\bar{X})$ such that $(\forall \bar{X})\left(\forall x_{1} \in X_{1}\right) \ldots\left(\forall x_{k} \in X_{k}\right)(\mathcal{P}(\bar{x}) \Leftrightarrow$ 
$\left.\left(x_{1}, \ldots, x_{k}\right) \in \mathcal{F}(\bar{X})\right)$ holds in $\mathfrak{M}$, as well as in $\mathcal{S}_{\mathfrak{M}}$. As for each $x \in \mathbb{S}_{\mathfrak{M}}$ there is $X \in \mathbb{S}_{\mathfrak{M}}$ with $x \in \mathfrak{M} X$ [for example, $X=\{x\}^{\mathfrak{M}}$ ], we have, for $\bar{x} \in \mathbb{S}_{\mathfrak{M}}, \mathcal{S}_{\mathfrak{M}} \vDash$ $\mathcal{P}(\bar{x}) \Leftrightarrow \mathcal{S}_{\mathfrak{M}} \vDash\left(x_{1}, \ldots, x_{k}\right) \in \mathcal{F}(\bar{X}) \Leftrightarrow \mathfrak{M} \vDash\left(x_{1}, \ldots, x_{k}\right) \in \mathcal{F}(\bar{X}) \Leftrightarrow \mathfrak{M} \vDash \mathcal{P}(\bar{x})$. This proves Transfer for restricted formulas.

If $\mathfrak{M} \vDash(\exists x) \mathcal{P}(x, \bar{x})$ for $\bar{x} \in \mathbb{S}_{\mathfrak{M}}$ and $\mathcal{P}(x, \bar{x})$ restricted, we use boundedness to fix $A \in \mathbb{S}_{\mathfrak{M}}$ such that $\mathfrak{M} \vDash(\exists x \in A) \mathcal{P}(x, \bar{x})$. Transfer for restricted formulas gives $\mathcal{S}_{\mathfrak{M}} \vDash(\exists x \in A) \mathcal{P}(x, \bar{x})$, and hence $\mathcal{S}_{\mathfrak{M}} \vDash(\exists x) \mathcal{P}(x, \bar{x})$. As the other direction is trivial, this proves Transfer for $\Sigma_{1}\left(\right.$ and $\left.\Pi_{1}\right)$ formulas.

We now assume that Transfer holds for $\Sigma_{n}$ and $\Pi_{n}$ formulas, $n \geq 1$, and prove it for $\Sigma_{n+1}$ formulas. Let $\mathfrak{M} \vDash(\exists x)(\forall y) \mathcal{P}(x, y, \bar{x})$ where $\bar{x} \in \mathbb{S}_{\mathfrak{M}}$ and $\mathcal{P}(x, y, \bar{x})$ is $\Sigma_{n-1}$. Again we fix $A \in \mathbb{S}_{\mathfrak{M}}$ such that $\mathfrak{M} \vDash(\exists x \in A)(\forall y) \mathcal{P}(x, y, \bar{x})$. It suffices to show that $\mathcal{S}_{\mathfrak{M}} \vDash(\exists x \in A)(\forall y) \mathcal{P}(x, y, \bar{x})$. If not, then $\mathcal{S}_{\mathfrak{M}} \vDash(\forall x \in A)(\exists y) \neg \mathcal{P}(x, y, \bar{x})$. $\mathcal{S}_{\mathfrak{M}}$ satisfies ZFC, and in particular Selection; hence there exists $f \in \mathbb{S}_{\mathfrak{M}}$ such that $\mathcal{S}_{\mathfrak{M}} \vDash(\forall x \in A) \neg \mathcal{P}(x, f(x), \bar{x})$. The latter is a $\Pi_{n}$ formula, therefore transfers to $\mathfrak{M}$ and contradicts $\mathfrak{M} \vDash(\exists x \in A)(\forall y) \mathcal{P}(x, y, \bar{x})$. The other direction is again trivial.

An embedding $\mathbb{F}$ of $\mathfrak{M}_{1}$ into $\mathfrak{M}_{2}$ is $\in$-elementary iff, for any $\in$-formula $\mathcal{P}(\bar{x})$,

$$
\left(\forall x_{1}, \ldots, x_{k} \in \mathbb{M}_{1}\right)\left(\mathfrak{M}_{1} \vDash \mathcal{P}\left(x_{1}, \ldots, x_{k}\right) \Leftrightarrow \mathfrak{M}_{2} \vDash \mathcal{P}\left(\mathbb{F}\left(x_{1}\right), \ldots, \mathbb{F}\left(x_{k}\right)\right)\right) .
$$

For bounded realizations this property is again expressible in $\mathbf{Z F C}$.

Proposition 1.3 Let $\mathfrak{M}_{1}, \mathfrak{M}_{2}$ be bounded realizations such that

$\mathfrak{M}_{j} \vDash(\forall x, y)(\exists ! z) \mathcal{P}_{i}(x, y, z)$ and

$\left(\forall x, y, z \in \mathbb{S}_{\mathfrak{M}_{j}}\right)\left(\mathfrak{M}_{j} \vDash \mathcal{P}_{i}(x, y, z) \Leftrightarrow \mathcal{S}_{\mathfrak{M}_{j}} \vDash \mathcal{P}_{i}(x, y, z)\right)(j=1,2 ; i=1, \ldots, 10)$.

If $\mathbb{F}$ is an embedding of $\mathfrak{M}_{1}$ into $\mathfrak{M}_{2}$ and

$\left(\forall x, y, z \in \mathbb{M}_{1}\right)\left(\mathfrak{M}_{1} \vDash \mathcal{P}_{i}(x, y, z) \Leftrightarrow \mathfrak{M}_{2} \vDash \mathcal{P}_{i}(\mathbb{F}(x), \mathbb{F}(y), \mathbb{F}(z))\right)(i=1, \ldots, 10)$,

then $\mathbb{F}$ is an $\in$-elementary embedding of $\mathfrak{M}_{1}$ into $\mathfrak{M}_{2}$.

Proof According to Proposition 1.2, $\mathfrak{M}_{1}$ satisfies $\mathbf{Z F C}$. We observe that $\mathbb{F}$ maps $\mathbb{S}_{\mathfrak{M}_{1}}$ onto $\mathbb{S}_{\mathfrak{M}_{2}}$; otherwise, there would exist a nontrivial $\in$-elementary embedding of $\mathcal{V}$ into $\mathcal{V}$, contradicting a famous theorem of Kunen (see Jech [19], Theorem 68). Hence for every $x \in \mathbb{M}_{2}$ there is $a \in \mathbb{M}_{1}$ (in fact, $a \in \mathbb{S}_{\mathfrak{M}_{1}}$ ) such that $x \in \mathfrak{M}_{2} \mathbb{F}(a)$. One can now repeat the argument from the proof of 1.2 with $\mathfrak{M}_{2}$ in place of $\mathfrak{M}$ and the image of $\mathbb{M}_{1}$ by $\mathbb{F}$ in place of $\mathbb{S}_{\mathfrak{M}}$. 
Let $\mathfrak{M}_{1}, \mathfrak{M}_{2}$ be realizations, with the language of $\mathfrak{M}_{1}$ included in the language of $\mathfrak{M}_{2} . \mathbb{F}$ is a morphism of $\mathfrak{M}_{1}$ to $\mathfrak{M}_{2}$ iff $\mathbb{F}$ is an embedding of $\mathfrak{M}_{1}$ into $\mathfrak{M}_{2}$ and is $\in$-elementary. We write $\mathfrak{M}_{1} \preccurlyeq \mathfrak{M}_{2}$ if $\mathbb{M}_{1} \subseteq \mathbb{M}_{2}$ and the identity on $\mathbb{M}_{1}$ is a morphism of $\mathfrak{M}_{1}$ to $\mathfrak{M}_{2}$, and read this “ $\mathfrak{M}_{2}$ is an elementary extension of $\mathfrak{M}_{1}$ ". Clearly, a bounded realization $\mathfrak{M}$ satisfies Transfer if and only if $\mathcal{S}_{\mathfrak{M}} \preccurlyeq \mathfrak{M}$.

Let $\mathfrak{M}$ and $\mathfrak{N}=\left(\mathbb{N},=\mathfrak{N}, \in_{\mathfrak{N}}, \mathbb{S}_{\mathfrak{N}}, \ldots\right)$ be interpretations and $\mathfrak{M} \vDash \mathbf{Z F C}$. Then one can consider the interpretation $\mathfrak{N}^{\mathfrak{M}}$ for the language of $\mathfrak{N}$, “ $\mathfrak{N}$ in the sense of $\mathfrak{M}$ ". It is obtained by taking the classes (formulas) that define $\mathfrak{N}$ and interpreting them in $\mathfrak{M}$ :

$$
\mathfrak{N}^{\mathfrak{M}}:=\left(\mathbb{N}^{\mathfrak{M}},(=\mathfrak{N})^{\mathfrak{M}},\left(\in_{\mathfrak{N}}\right)^{\mathfrak{M}},\left(\mathbb{S}_{\mathfrak{N}}\right)^{\mathfrak{M}}, \ldots\right)
$$

The following proposition is easily verified by induction on complexity of formulas.

Proposition 1.4 Let $\mathcal{P}\left(x_{1}, \ldots, x_{k}\right)$ be any formula in the language of $\mathfrak{N}$. For all $x_{1}, \ldots, x_{k} \in \mathbb{N}^{\mathfrak{M}}, \quad \mathfrak{N}^{\mathfrak{M}} \vDash \mathcal{P}\left(x_{1}, \ldots, x_{k}\right)$ iff $\mathfrak{M} \vDash\left(\mathfrak{N} \vDash \mathcal{P}\left(x_{1}, \ldots, x_{k}\right)\right)$.

In particular, if $\mathcal{Q}$ is a sentence expressing the fact that $=$ is a congruence (with respect to the language of $\mathfrak{N}$ ), then (ZFC proves that) $\mathfrak{N} \vDash \mathcal{Q}$ and, as $\mathfrak{M} \vDash \mathbf{Z F C}$ is assumed, $\mathfrak{M} \vDash(\mathfrak{N} \vDash \mathcal{Q})$ and $\mathfrak{N}^{\mathfrak{M}} \vDash \mathcal{Q}$. So $\mathfrak{N}^{\mathfrak{M}}$ is indeed an interpretation. [We note that it need not be realistic even when $\mathfrak{M}$ and $\mathfrak{N}$ are.]

Let $\mathbb{F}$ be an embedding of $\mathfrak{N}_{1}$ into $\mathfrak{N}_{2}$ and $\mathfrak{M} \vDash \mathbf{Z F C}$. Then $\mathbb{F}^{\mathfrak{M}}$ is an embedding of $\mathfrak{N}_{1}^{\mathfrak{M}}$ into $\mathfrak{N}_{2}^{\mathfrak{M}}$. If $\mathbb{F}$ is an isomorphism, then $\mathbb{F}^{\mathfrak{M}}$ is an isomorphism.

Conversely, let $\mathfrak{M} \vDash$ " $\mathbb{F}$ is an embedding (resp., isomorphism) of $\mathfrak{N}_{1}$ into $\mathfrak{N}_{2}$ ". Then $\mathbb{F} \uparrow \mathbb{N}_{1}^{\mathfrak{M}}$ induces an embedding (resp., isomorphism) of $\mathfrak{N}_{1}^{\mathfrak{M}}$ into $\mathfrak{N}_{2}^{\mathfrak{M}}$ in an obvious way.

Another easy induction shows that $\left.\mathfrak{L}^{\left[\mathfrak{M}^{\mathfrak{M}}\right.}\right]=\left[\mathfrak{L}^{\mathfrak{N}}\right]^{\mathfrak{M}}$.

Let $\mathbb{F}$ be a morphism of a realization $\mathfrak{M}_{1}$ to a realization $\mathfrak{M}_{2}$ and $\mathfrak{M}_{1} \vDash \mathbf{Z F C}$ (hence $\mathfrak{M}_{2} \vDash \mathbf{Z F C}$ as well). If $\mathfrak{N}=\left(\mathbb{N},=\mathfrak{N}, \in \mathfrak{N}, \mathbb{S}_{\mathfrak{N}}, \ldots\right)$ is an interpretation, we can form $\mathfrak{N}^{\mathfrak{M}_{1}}$ and $\mathfrak{N}^{\mathfrak{M}_{2}}$; it is immediate that $\mathbb{F} \uparrow \mathbb{N}^{\mathfrak{M}_{1}}$ is a morphism of $\mathfrak{N}^{\mathfrak{M}_{1}}$ into $\mathfrak{N}^{\mathfrak{M}_{2}}$. The especially useful case is when $\mathfrak{M}_{1}$ is $\mathcal{S}_{\mathfrak{M}}, \mathfrak{M}_{2}$ is $\mathfrak{M}$, and $\mathbb{F}$ is the identity on $\mathbb{S}_{\mathfrak{M}}$; we get $\mathbb{N}^{\mathcal{S}_{\mathfrak{M}}}=\mathbb{N}^{\mathfrak{M}} \cap \mathbb{S}_{\mathfrak{M}} ;(=\mathfrak{N})^{\mathcal{S}_{\mathfrak{M}}}$ is $(=\mathfrak{N})^{\mathfrak{M}} \cap\left(\mathbb{S}_{\mathfrak{M}_{1}} \times \mathbb{S}_{\mathfrak{M}_{1}}\right) ;$ similarly for $\epsilon_{\mathfrak{N}}$; $\left(\mathbb{S}_{\mathfrak{N}}\right)^{\mathcal{S}_{\mathfrak{M}}}=\left(\mathbb{S}_{\mathfrak{N}}\right)^{\mathfrak{M}} \cap \mathbb{S}_{\mathfrak{M}}$

We conclude this section with a list of some particular notational conventions.

As already mentioned, letters in blackboard, calligraphic, gothic, and uppercase Greek types denote classes. $k, \ell, m, n$ are natural numbers; $\kappa, \lambda$ are infinite cardinals; $I d_{A}$ is 
the identity function on $A$ ( $\mathbb{I} d_{\mathbb{A}}$ if $\mathbb{A}$ is a class); $f, g, h, F, G, H$ are always functions. $f: \subseteq I \rightarrow J$ means that $\operatorname{dom} f \subseteq I$ and $\operatorname{ran} f \subseteq J . \mathbb{F}\lceil A$ is $\{\langle x, y\rangle \in \mathbb{F}: x \in A\}$, the restriction of $\mathbb{F}$ to $A$. 0 is the empty set as well as the number zero. $\mathbb{V}$ is the class of all sets. $\mathbb{V}^{A}=\{f: \operatorname{dom} f=A\}$; a special convention is introduced in Section 2 in case $A$ is an ultrafilter. We maintain a distinction between ordered pairs $(a, b)$ and 2-tuples $\langle a, b\rangle$; it is of course unimportant for most purposes. The elements of $\mathbb{V}^{k}$ are $k$-tuples, and we denote them by $\left\langle x_{1}, \ldots, x_{k}\right\rangle$. The projections $\pi_{i}^{k}: \mathbb{V}^{k} \rightarrow \mathbb{V}$ are defined for $1 \leq i \leq k$ by $\pi_{i}^{k}\left(\left\langle x_{1}, \ldots, x_{k}\right\rangle\right)=x_{i}$; we omit $k$ when it is clear from the context. $A_{1} \times \ldots \times A_{k}$ is viewed as a subset of $\mathbb{V}^{k}$.

The symbol := denotes "equal by definition". Quotation marks in mathematical context are used as parentheses.

\section{Ultrafilters and Ultrapowers.}

Ultrafilters are pervasive in set theory, and the ultrapower construction is one of the key tools in the instrumentarium of model theory. The classic references are ComfortNegrepontis [7] and Chang-Keisler [6].

Definition 2.1 $U$ is an ultrafilter over $I$ iff $U \subseteq \mathcal{P}(I)$ and

(i) $0 \notin U ; I \in U$

(ii) $(\forall X, Y \in U)(X \cap Y \in U)$

(iii) $(\forall X, Y \in U)(X \cup Y \in U \Rightarrow X \in U \vee Y \in U)$.

It follows that $(\forall X \in U)(\forall Y \subseteq I)(X \subseteq Y \Rightarrow Y \in U)$ and $(\forall X \subseteq I)(X \in U$ $\vee I \backslash X \in U$ ). We note that $I$ is determined by $U: I=\bigcup U$; we call $I$ the domain of $U$ and denote it $\operatorname{dom} U$.

For $X \in U, U \uparrow X:=U \cap \mathcal{P}(X)$ is an ultrafilter over $X$

An ultrafilter is principal iff $\{x\} \in U$ for some $x \in \operatorname{dom} U$. If such $x$ exists, it is uniquely determined, and $U \uparrow\{x\}=\{\{x\}\}$ is then an ultrafilter over $\{x\}$. We use $W_{x, I}$ to denote the principal ultrafilter over $I$ that contains $\{x\}$.

Letters $U, V$, and $W$ are reserved for ultrafilters. We write $U \sim V$ iff $U \cap V$ is an ultrafilter, ie, $(\exists X \in U \cap V)(U\lceil X=V\lceil X) ; \sim$ is an equivalence relation.

The technical subject of this work is detailed study of ultrapowers of the universe $\mathbb{V}$ of set theory, and of direct limits of systems of such ultrapowers. Constructions of this 
kind play an important role in numerous investigations in set theory; we mention only Scott [32], Vopěnka [34], Gaifman [9] and Kunen [26].

We let $\mathbb{V}^{U}:=\{f: f$ is a function, $\operatorname{dom} f \in U\}$, and for $f, g \in \mathbb{V}^{U}$ define

$$
\begin{aligned}
& f={ }_{U} g \text { iff }\{i \in I: f(i)=g(i)\} \in U ; \\
& f \in_{U} g \text { iff }\{i \in I: f(i) \in g(i)\} \in U .
\end{aligned}
$$

The ultrapower of $\mathbb{V}$ modulo $U$ is the interpretation $\mathcal{U} \ell(\mathbb{V} ; U):=\left(\mathbb{V}^{U},=_{U}, \in_{U}\right)$.

The universe of $\mathcal{U} \ell(\mathbb{V} ; U)$ is a proper class, and the satisfaction relation cannot be defined for this "structure" (it would induce one for $(\mathbb{V}, \in)$ ). But, if $\mathcal{P}(\bar{x})$ is any $\in$ formula, we let $\mathcal{U} \ell(\mathbb{V} ; U) \vDash \mathcal{P}(\bar{x})$ denote the formula $\mathcal{P}^{\mathcal{U} \ell(\mathbb{V} ; U)}(\bar{x})$ obtained from $\mathcal{P}$ by restricting the range of all quantifiers to $\mathbb{V}^{U}$, and replacing all occurrences of $=$ and $\in$ by $=_{U}$ and $\epsilon_{U}$, resp. [This may involve renaming some bound variables, if necessary or convenient.] The fundamental fact about ultrapowers now takes the following form.

Proposition 2.2 (Łoś Theorem) Let $\mathcal{P}\left(x_{1}, \ldots, x_{k}\right)$ be an $\in$-formula.

For all $f_{1}, \ldots, f_{k} \in \mathbb{V}^{U}$,

$$
\mathcal{U} \ell(\mathbb{V} ; U) \vDash \mathcal{P}\left(f_{1}, \ldots, f_{k}\right) \Leftrightarrow\left\{i \in I: \mathcal{P}\left(f_{1}(i), \ldots, f_{k}(i)\right)\right\} \in U .
$$

Proof By induction on the complexity of $\mathcal{P}$.

If $\mathcal{P}$ is an atomic formula $x_{\ell}=x_{m}, \mathcal{U} \ell(\mathbb{V} ; U) \vDash f_{\ell}=f_{m}$ is the formula $f_{\ell}={ }_{U} f_{m}$, which holds if and only if $\left\{i \in I: f_{\ell}(i)=f_{m}(i)\right\} \in U$.

The case of $x_{\ell} \in x_{m}$ is similar, and propositional connectives $(\neg, \wedge)$ are trivial.

If $\mathcal{P}$ is of the form $(\exists y) \mathcal{Q}\left(x_{1}, \ldots, x_{k}, y\right), \mathcal{U} \ell(\mathbb{V} ; U) \vDash(\exists y) \mathcal{Q}\left(f_{1}, \ldots, f_{k}, y\right)$ is the formula $\left(\exists g \in \mathbb{V}^{U}\right)\left(\mathcal{U} \ell(\mathbb{V} ; U) \vDash \mathcal{Q}\left(f_{1}, \ldots, f_{k}, g\right)\right)$. Let $g \in \mathbb{V}^{U}$ be such that $\mathcal{U} \ell(\mathbb{V} ; U) \vDash$ $\mathcal{Q}\left(f_{1}, \ldots, f_{k}, g\right)$. By the inductive assumption, $\left\{i \in I: \mathcal{Q}\left(f_{1}(i), \ldots, f_{k}(i), g(i)\right)\right\} \in U$, hence $\left\{i \in I:(\exists x) \mathcal{Q}\left(f_{1}(i), \ldots, f_{k}(i), x\right)\right\} \in U$, ie, $\left\{i \in I: \mathcal{P}\left(f_{1}(i), \ldots, f_{k}(i)\right)\right\} \in U$. Using Selection, the argument can be reversed.

In particular, $(\mathcal{U} \ell(\mathbb{V} ; U) \vDash \mathcal{P}) \Leftrightarrow \mathcal{P}$ holds for any sentence $\mathcal{P}$, so $\mathcal{U} \ell(\mathbb{V} ; U)$ is an interpretation for the $\in$-language and satisfies all axioms of $\mathbf{Z F C}$.

The ultrapower construction is easily extended to "structures" for richer languages. For example, let $\mathbb{R} \subseteq \mathbb{V}^{2}$. We let $\mathbb{R}_{U}:=\left\{\langle f, g\rangle \in \mathbb{V}^{U} \times \mathbb{V}^{U}:\{i \in I:\langle f(i), g(i)\rangle \in\right.$ $\mathbb{R}\} \in U\}$, and define $\mathcal{U} \ell(\mathbb{V} ; \mathbb{R} ; U):=\left(\mathbb{V}^{U},=_{U}, \in_{U}, \mathbb{R}_{U}\right)$. As the class $\mathbb{R}$ is defined by some $\in$-formula, Łoś Theorem is easily seen to hold for $\mathcal{U} \ell(\mathbb{V} ; \mathbb{R} ; U)$ and formulas in the language with $=, \in$, and an additional binary relation symbol $R$ interpreted as $\mathbb{R}$. 
Our main emphasis is on ultrapowers of the universe, but occasionally we need the more general concept of ultraproduct. Let $\mathbb{M}, \mathbb{I}, \mathbb{E} \subseteq I \times \mathbb{V}$; we think of $\mathbb{M}$ as a system of classes $\left\langle\mathbb{M}_{i}: i \in I\right\rangle$ indexed by $I$, where $\mathbb{M}_{i}:=(\mathbb{M})_{i}=\{x:\langle i, x\rangle \in \mathbb{M}\}$; similarly for $\left\langle\mathbb{I}_{i}: i \in I\right\rangle$ and $\left\langle\mathbb{E}_{i}: i \in I\right\rangle$. Assume that for each $i \in I,\left(\mathbb{M}_{i}, \mathbb{I}_{i}, \mathbb{E}_{i}\right)$ is an interpretation for the $\in$-language. We let

$\prod_{i \in I} \mathbb{M}_{i} / U:=\left\{f \in \mathbb{V}^{U}:\left\{i \in \operatorname{dom} f: f(i) \in \mathbb{M}_{i}\right\} \in U\right\}$,

$\prod_{i \in I} \mathbb{I}_{i} / U:=\left\{\langle f, g\rangle \in \mathbb{V}^{U} \times \mathbb{V}^{U}:\left\{i:\langle f(i), g(i)\rangle \in \mathbb{I}_{i}\right\} \in U\right\}$,

and similarly for $\prod_{i \in I} \mathbb{E}_{i} / U$. Then the ultraproduct $\mathcal{U} \ell\left(\left\langle\mathbb{M}_{i}, \mathbb{I}_{i}, \mathbb{E}_{i}\right\rangle_{i \in I} ; U\right)$ is defined as $\left(\prod_{i \in I} \mathbb{M}_{i} / U, \prod_{i \in I} \mathbb{I}_{i} / U, \prod_{i \in I} \mathbb{E}_{i} / U\right)$. Łoś Theorem holds in the form

$$
\mathcal{U} \ell\left(\left\langle\mathbb{M}_{i}, \mathbb{I}_{i}, \mathbb{E}_{i}\right\rangle ; U\right) \vDash \mathcal{P}\left(f_{1}, \ldots, f_{k}\right) \Leftrightarrow\left\{i:\left(\mathbb{M}_{i}, \mathbb{I}_{i}, \mathbb{E}_{i}\right) \vDash \mathcal{P}\left(f_{1}(i), \ldots, f_{k}(i)\right)\right\} \in U .
$$

Lemma 2.3 Let $U_{1}\left(U_{2}\right.$, resp.) be an ultrafilter over $I_{1}\left(I_{2}\right.$, resp. $)$ and let $\varphi$ be a function. The following statements are equivalent:

$$
\begin{gathered}
\left(\forall X \in U_{1}\right)\left(\varphi^{-1}[X] \cap I_{2} \in U_{2}\right) \\
\left(\forall Y \in U_{2}\right)\left(\varphi[Y] \cap I_{1} \in U_{1}\right) .
\end{gathered}
$$

Proof $(1) \Rightarrow(2)$ :

Assume $Y \in U_{2}$ and let $X:=\varphi[Y] \cap I_{1}$. If $X \notin U_{1}$ then $I_{1} \backslash X \in U_{1}$, so by (1), $\varphi^{-1}\left[I_{1} \backslash X\right] \cap I_{2} \in U_{2}$. But $\varphi^{-1}\left[I_{1} \backslash X\right]$ is disjoint with $Y \in U_{2}$, a contradiction.

$(2) \Rightarrow(1)$ :

Assume $X \in U_{1}$ and let $Y:=\varphi^{-1}[X] \cap I_{2}$. If $Y \notin U_{2}$, then $I_{2} \backslash Y \in U_{2}$, so by (2), $\varphi\left[I_{2} \backslash Y\right] \cap I_{1} \in U_{1}$. But $\varphi\left[I_{2} \backslash Y\right]$ is disjoint with $X \in U_{1}$, a contradiction.

A morphism from $U_{2}$ to $U_{1}$ is a function $\varphi: \subseteq I_{2} \rightarrow I_{1}$ such that (1) or (2) holds; notation $\varphi: U_{2} \rightarrow U_{1}$. Observe that $\operatorname{dom} \varphi=\varphi^{-1}\left[I_{1}\right] \in U_{2}$.

If $U_{2}$ is an ultrafilter and $\varphi: \subseteq I_{2} \rightarrow I_{1}$, we let

$$
\varphi\left[U_{2}\right]:=\left\{X \subseteq I_{1}: \varphi^{-1}[X] \in U_{2}\right\}=\left\{X \subseteq I_{1}: X \supseteq \varphi[Y] \text { for some } Y \in U_{2}\right\} .
$$

We then have $\varphi: U_{2} \rightarrow \varphi\left[U_{2}\right]$; conversely, if $\varphi: U_{2} \rightarrow U_{1}$, then $U_{1}=\varphi\left[U_{2}\right]$.

Evidently, if $\varphi: U_{2} \rightarrow U_{1}$ and $\psi: U_{3} \rightarrow U_{2}$, then $\varphi \circ \psi: U_{3} \rightarrow U_{1}$. Hence $U_{1} \leq_{R K} U_{2}$ iff $(\exists \varphi)\left(\varphi: U_{2} \rightarrow U_{1}\right)$ defines a preordering of ultrafilters, called the Rudin-Keisler preordering. The well-known theorem first proved by Katětov (see [7]) asserts that, if $\varphi: U \rightarrow U$, then $\varphi={ }_{U} I d_{I}$. It follows that, if $U_{1} \leq_{R K} U_{2}$ and 
$U_{2} \leq_{R K} U_{1}$, then there is a one-one function $g: \subseteq I_{1} \rightarrow I_{2}$ such that $g\left[U_{1}\right]=U_{2}$ and $g^{-1}\left[U_{2}\right]=U_{1}$. We call such $g$ an isomorphism of $U_{1}$ and $U_{2}$.

Every morphism of ultrafilters induces an $\in$-elementary embedding of the corresponding ultrapowers.

Let $\varphi: U_{2} \rightarrow U_{1}$; we define $\varphi^{*}: \mathbb{V}^{U_{1}} \rightarrow \mathbb{V}^{U_{2}}$ by $\varphi^{*}(f)=f \circ \varphi$.

Evidently, if $\varphi: U_{2} \rightarrow U_{1}$ and $\psi: U_{3} \rightarrow U_{2}$ then $(\varphi \circ \psi)^{*}=\psi^{*} \circ \varphi^{*}$.

If $U_{1} \sim U_{2}$ and $\varphi=I d_{X}$ where $X \in U_{1} \cap U_{2}$, then $\varphi^{*}$ is an isomorphism of $\mathcal{U} \ell\left(\mathbb{V} ; U_{1}\right)$ and $\mathcal{U} \ell\left(\mathbb{V} ; U_{2}\right)$.

Proposition 2.4 Let $\mathcal{P}\left(x_{1}, \ldots, x_{k}\right)$ be an $\in$-formula. For all $f_{1}, \ldots, f_{k} \in \mathbb{V}^{U_{1}}$,

$$
\mathcal{U} \ell\left(\mathbb{V} ; U_{1}\right) \vDash \mathcal{P}\left(f_{1}, \ldots, f_{k}\right) \Leftrightarrow \mathcal{U} \ell\left(\mathbb{V} ; U_{2}\right) \vDash \mathcal{P}\left(\varphi^{*}\left(f_{1}\right), \ldots, \varphi^{*}\left(f_{k}\right)\right) .
$$

Proof We have $\mathcal{U} \ell\left(\mathbb{V} ; U_{1}\right) \vDash \mathcal{P}\left(f_{1}, \ldots, f_{k}\right)$ iff $\left\{i \in I_{1}: \mathcal{P}\left(f_{1}(i), \ldots, f_{k}(i)\right)\right\} \in U_{1}$ iff $\varphi^{-1}\left[\left\{i \in I_{1}: \mathcal{P}\left(f_{1}(i), \ldots, f_{k}(i)\right)\right\}\right] \in U_{2}$ iff $\left\{j \in I_{2}: \mathcal{P}\left(f_{1}(\varphi(j)), \ldots, f_{k}(\varphi(j))\right)\right\} \in U_{2}$ iff $\left\{j \in I_{2}: \mathcal{P}\left(\varphi^{*}\left(f_{1}\right)(j), \ldots, \varphi^{*}\left(f_{k}\right)(j)\right)\right\} \in U_{2}$ iff $\mathcal{U} \ell\left(\mathbb{V} ; U_{2}\right) \vDash \mathcal{P}\left(\varphi^{*}\left(f_{1}\right), \ldots, \varphi^{*}\left(f_{k}\right)\right)$.

The converse assertion, that every $\in$-elementary embedding of $\mathcal{U} \ell\left(\mathbb{V} ; U_{1}\right)$ to $\mathcal{U} \ell\left(\mathbb{V} ; U_{2}\right)$ is induced by some morphism from $U_{2}$ to $U_{1}$, cannot be proved in ZFC (see [3, 15]). The induced embeddings are special, in that they commute with the natural $\in$-elementary embeddings of $\mathbb{V}$ into $\mathcal{U} \ell\left(\mathbb{V} ; U_{1}\right)$ and $\mathcal{U} \ell\left(\mathbb{V} ; U_{2}\right)$.

Let $I_{0}=\{0\}$ and let $U_{0}$ be the principal ultrafilter over $I_{0}$; ie, $U_{0}=\{\{0\}\}$. There is a natural isomorphism $\mathfrak{k}_{0}$ between $(\mathbb{V},=, \in)$ and $\mathcal{U} \ell\left(\mathbb{V} ; U_{0}\right)$ given by $\mathfrak{k}_{0}(x)=\{(0, x)\}$. If $U$ is an ultrafilter over $I$ and $\varphi_{U}: U \rightarrow U_{0}$ is defined by $\varphi_{U}(i)=0$ for all $i \in I$, we call $\mathfrak{k}_{U}:=\varphi_{U}^{*} \circ \mathfrak{k}_{0}$ the natural embedding of $(\mathbb{V},=, \in)$ into $\mathcal{U} \ell(\mathbb{V} ; U)$.

Unraveling this definition shows that, for every $x \in \mathbb{V}, \mathfrak{k}_{U}(x)=c_{x}$, where $c_{x}$ is the constant function on $I$ with value $x$ : $\mathfrak{k}_{U}(x)(i)=c_{x}(i)=x$ for all $i \in I$. [ $\mathfrak{k}_{U}$ is also known as the diagonal mapping.]

If $\varphi: U_{2} \rightarrow U_{1}$, then $\varphi_{U_{1}} \circ \varphi=\varphi_{U_{2}}$, hence $\mathfrak{k}_{U_{2}}=\varphi_{U_{2}}^{*} \circ \mathfrak{k}_{0}=\varphi^{*} \circ \varphi_{U_{1}}^{*} \circ \mathfrak{k}_{0}=\varphi^{*} \circ \mathfrak{k}_{U_{1}}$.

In summary: The $\in$-elementary embeddings induced by morphisms commute with the natural embeddings.

Proposition 2.5 [Keisler; Lindstrom; [6]] Let $\Psi: \mathcal{U} \ell\left(\mathbb{V} ; U_{1}\right) \rightarrow \mathcal{U} \ell\left(\mathbb{V} ; U_{2}\right)$ be an $\in$-elementary embedding such that $\Psi \circ \mathfrak{k}_{U_{1}}=\mathfrak{k}_{U_{2}}$. Then there is $\varphi: U_{2} \rightarrow U_{1}$ such that $\Psi=\varphi^{*}$. If also $\widetilde{\varphi}: U_{2} \rightarrow U_{1}$ and $\Psi=\widetilde{\varphi}^{*}$, then $\varphi={ }_{U_{2}} \widetilde{\varphi}$. 
Proof Let $d:=I d_{I_{1}}$ be the identity on $I_{1}$. We set $e:=\Psi(d) \in \mathbb{V}^{U_{2}}$, and write $\mathfrak{k}_{1}, \mathfrak{k}_{2}$ in place of $\mathfrak{k}_{U_{1}}, \mathfrak{k}_{U_{2}}$.

Claim 1. $A \in U_{1}$ implies $e^{-1}[A] \in U_{2}$.

Proof $d(i)=i \in A$ for all $i \in A \in U_{1}$, so $d \in_{U_{1}} \mathfrak{k}_{1}(A)$. Hence $e=\Psi(d) \in_{U_{2}}$ $\Psi\left(\mathfrak{k}_{1}(A)\right)=\mathfrak{k}_{2}(A)$, ie, $\left\{j \in I_{2}: e(j) \in A\right\}=e^{-1}[A] \in U_{2}$.

We let $\varphi:=e\left\lceil e^{-1}\left[I_{1}\right]\right.$, so $\varphi={ }_{U_{2}} e$ and $\operatorname{ran} \varphi \subseteq I_{1}$. Claim 1 remains true with $e$ replaced by $\varphi$, and shows that $\varphi: U_{2} \rightarrow U_{1}$.

Claim 2. $\varphi^{*}(f)={ }_{U_{2}} \Psi(f)$ for all $f \in \mathbb{V}^{U_{1}}$.

Proof We observe that, for all $i \in \operatorname{dom} f, f(i)=f(d(i))=\left(\mathfrak{k}_{1}(f)(i)\right)(d(i))$, so $\mathcal{U} \ell\left(\mathbb{V} ; U_{1}\right) \vDash f=\mathfrak{k}_{1}(f)(d)$. As $\Psi$ is an $\in$-elementary embedding, we get $\mathcal{U} \ell\left(\mathbb{V} ; U_{2}\right) \vDash$ “ $\Psi(f)=\Psi\left(\mathfrak{k}_{1}(f)\right)(\Psi(d))=\mathfrak{k}_{2}(f)(\varphi)=f \circ \varphi$ ", and conclude that $\Psi(f)={ }_{U_{2}} f \circ \varphi=$ $\varphi^{*}(f)$. [We recall that the underlined composition $f \circ \varphi$ is evaluated in the ambient set theory, not in $\mathcal{U} \ell\left(\mathbb{V} ; U_{2}\right)$; hence the meaning is $\mathcal{U} \ell\left(\mathbb{V} ; U_{2}\right) \vDash \Psi(f)=g$ where $g:=f \circ \varphi$.]

If $\varphi^{*}=\widetilde{\varphi}^{*}$, then in particular $\varphi^{*}(d)={ }_{U_{2}} \widetilde{\varphi}^{*}(d)$; but $\varphi^{*}(d)=d \circ \varphi=\varphi, \widetilde{\varphi}^{*}(d)=$ $d \circ \widetilde{\varphi}^{*}=\widetilde{\varphi}^{*}$, so $\varphi={ }_{U_{2}} \widetilde{\varphi}^{*}$.

It is not clear a priori that Proposition 2.5 is a theorem of $\mathbf{Z F C}$, because the notion of elementary embedding is defined by a schema of equivalences: for every $\in$-formula $\mathcal{P}, \mathcal{U} \ell\left(\mathbb{V} ; U_{1}\right) \vDash \mathcal{P}\left(f_{1}, \ldots, f_{k}\right)$ iff $\mathcal{U} \ell\left(\mathbb{V} ; U_{2}\right) \vDash \mathcal{P}\left(\Psi\left(f_{1}\right), \ldots, \Psi\left(f_{k}\right)\right)$. However, an inspection of its proof reveals that a single equivalence, for the formula $\mathcal{P}_{0}(x, y, z)$ : " $y$ is a function and $z=y(x)$ " [with $x=d, y=\mathfrak{k}_{1}(f)$ and $z=f$ ] suffices to prove that $\Psi=\varphi^{*}$. As $\varphi^{*}$ is an $\in$-elementary embedding, so is $\Psi$. Hence, "an embedding $\Psi: \mathcal{U} \ell\left(\mathbb{V} ; U_{1}\right) \rightarrow \mathcal{U} \ell\left(\mathbb{V} ; U_{2}\right)$ such that $\Psi \circ \mathfrak{k}_{U_{1}}=\mathfrak{k}_{U_{2}}$ and $\left(\forall f, g, h \in \mathbb{V}^{U_{1}}\right)\left(\mathcal{U} \ell\left(\mathbb{V} ; U_{1}\right) \vDash\right.$ $\mathcal{P}_{0}(f, g, h) \Leftrightarrow \mathcal{U} \ell\left(\mathbb{V} ; U_{2}\right) \vDash \mathcal{P}_{0}(\Psi(f), \Psi(g), \Psi(h))$ " can be taken as a definition of " $\in-$ elementary embedding" in Proposition 2.5.

Proposition 2.5 suggests that ultrapowers of $\mathbb{V}$ should be construed as realizations. To that effect we add to the $\in$-language a unary predicate symbol st, to be interpreted by the class $\mathbb{S}_{U}:=\mathfrak{k}_{U}[\mathbb{V}]=\left\{f \in \mathbb{V}^{U}: f={ }_{U} \mathfrak{k}_{U}(x)\right.$ for some $\left.x \in \mathbb{V}\right\}$, the range of the natural embedding $\mathfrak{k}_{U}$. From now on, the ultrapower of $\mathbb{V}$ modulo $U$ is the interpretation $\mathcal{U} \ell t(\mathbb{V} ; U):=\left(\mathbb{V}^{U},=_{U}, \in_{U}, \mathbb{S}_{U}\right)$ for the $\{\in, \mathbf{s t}\}$-language. The natural isomorphism $\mathfrak{k}_{U}$ of $(\mathbb{V},=, \in)$ and $\left(\mathbb{S}_{U},={ }_{U} \cap\left(\mathbb{S}_{U} \times \mathbb{S}_{U}\right), \in_{U} \cap\left(\mathbb{S}_{U} \times \mathbb{S}_{U}\right)\right)$ witnesses that $\left(\mathcal{U} \ell t(\mathbb{V} ; U), \mathfrak{k}_{U}\right)$ is a realization. Proposition 1.1 tells us that, in $\mathbf{Z F C}, \mathfrak{k}_{U}$ is uniquely determined by the class $\mathbb{S}_{U}$. 
Let $\mathcal{U} \ell t\left(\mathbb{V} ; U_{1}\right)$ and $\mathcal{U} \ell t\left(\mathbb{V} ; U_{2}\right)$ be ultrapowers of $\mathbb{V} . \Psi: \mathcal{U} \ell t\left(\mathbb{V} ; U_{1}\right) \rightarrow \mathcal{U} \ell t\left(\mathbb{V} ; U_{2}\right)$ denotes that $\Psi$ is a morphism in the sense of realizations; that is, $\Psi$ is an $\in$-elementary embedding and $\Psi \circ \mathfrak{k}_{U_{1}}=\mathfrak{k}_{U_{2}}$.

Proposition 1.3, or, alternatively, the remarks after the proof of Proposition 2.5, show that this notion is expressible in ZFC. Moreover, every morphism is of the form $\varphi^{*}$ for some $\varphi: U_{2} \rightarrow U_{1}$, so we have a "duality" between the category of ultrafilters and that of ultrapowers of the universe. (This statement is not quite correct in the technical sense of category theory, due - among other things - to the fact that $\varphi$ is unique only almost everywhere.)

\section{Nonstandard Set Theory.}

Our goal here is a sound and complete axiomatization of the interpretations $\mathcal{U} \ell t(\mathbb{V} ; U)=$ $\left(\mathbb{V}^{U},=_{U}, \in_{U}, \mathbb{S}_{U}\right)$ for the language that has, in addition to $=$ and $\in$, also a unary predicate symbol st, interpreted by $\mathbb{S}_{U}$. It is customary to read $\mathbf{s t}(x)$ as " $x$ is standard." Elements of $\mathbb{S}_{U}$ are the standard sets in $\mathcal{U} \ell t(\mathbb{V} ; U)$; if $U$ is nonprincipal, then $I d_{I} \neq_{U} \mathfrak{k}_{U}(x)$ for all $x \in \mathbb{V}$, and $\mathcal{U} \ell t(\mathbb{V} ; U) \vDash(\exists x)(\neg \mathbf{s t}(x))$. Hence nonstandard sets exist in $\mathcal{U} \ell t(\mathbb{V} ; U)$. Nonstandard set theory axiomatizes ultrapowers of the universe. ${ }^{2}$

We use $\left(\exists^{\text {st }} x\right) \ldots$ as shorthand for $(\exists x)(\operatorname{st}(x) \wedge \ldots)$, and $\left(\forall^{\text {st }} x\right) \ldots$ as shorthand for $(\forall x)(\mathbf{s t}(x) \Rightarrow \ldots) . \mathcal{P}^{\text {st }}$ is a formula obtained from $\mathcal{P}$ by replacing each occurrence of $(\exists x) \ldots$ by $\left(\exists^{\text {st }} x\right) \ldots$, and of $(\forall x) \ldots$ by $\left(\forall^{\text {st }} x\right) \ldots$

Elementary nonstandard set theory $\mathbf{S T}^{-}$is the theory in the $\{\in$, st $\}$-language with the following axioms:

ZFC for standard sets: $\mathcal{P}^{\text {st }}$, where $\mathcal{P}$ is any axiom of $\mathbf{Z F C}$.

Boundedness: $\quad(\forall x)\left(\exists^{\text {st }} a\right)(x \in a)$.

Transfer: $\quad\left(\forall^{\text {st }} x_{1}, \ldots, x_{k}\right)\left(\mathcal{P}^{\text {st }}\left(x_{1}, \ldots, x_{k}\right) \Leftrightarrow \mathcal{P}\left(x_{1}, \ldots, x_{k}\right)\right)$, where $\mathcal{P}$ is any $\in$-formula.

\footnotetext{
${ }^{2}$ In a private conversation, J Väänänen asked, how does nonstandard set theory differ from studying a $\kappa$-saturated ultrapower of $V_{\kappa}$ where $\kappa$ is inaccessible. During my talk at CUNY Graduate Center on an early version of this material, G Liebman asked, how do models of nonstandard set theory differ from nonstandard models of set theory. The discussion at the end of the preceding section is the beginning of an answer to these questions: $\mathcal{U} \ell(\mathbb{V}, U)$ is a nonstandard "model" of set theory, while $\mathcal{U} \ell t(\mathbb{V} ; U)$ is a "model" of nonstandard set theory. In other words, in nonstandard set theory one always considers also the primitive predicate st, or, equivalently, a particular elementary embedding of $\mathbb{V}$ into $\mathbb{V}^{U}$.
} 
We let $\mathbb{S}:=\{x: \mathbf{s t}(x)\}$ be the class of standard sets and $\mathbb{V}:=\{x: x=x\}$ the class of all sets, sometimes referred to as internal sets for emphasis. A consequence of Transfer is that $\mathbf{Z F C}$ holds ( for internal sets).

ST is $\mathbf{S T}^{-}$plus

Inner Standardization: $\quad(\forall x)\left(\exists^{\text {st }} a\right)\left(\forall^{\text {st }} z\right)(z \in a \Leftrightarrow z \in x)$.

The standard set $a$ in the Inner Standardization axiom is uniquely determined by $x$; we denote it $\mathbf{s h}(x)$ and call it the shadow of $x$.

Remarks (1) Every realization of $\mathbf{S T}^{-}$is bounded.

(2) In ST, Boundedness is a consequence of the remaining axioms.

Proof The axiom of Regularity implies that for every set $x$ there is an ordinal $\xi$ such that $x \in \mathbb{V}_{\xi}$, the $\xi$ 'th level of the cumulative hierarchy. It suffices to show that there is an ordinal $\eta \in \mathbb{S}, \eta \geq \xi$; then $x \in \mathbb{V}_{\eta} \in \mathbb{S}$. If not, then $\xi>\eta$ for all ordinals $\eta \in \mathbb{S}$ and $\mathbf{s h}(\xi) \in \mathbb{S}$ is a standard set containing all standard ordinals, contradicting $\mathrm{ZFC}^{\text {st }}$.

Proposition 3.1 $[6,22] \mathcal{U} \ell t(\mathbb{V} ; U)$ is a realization of $\mathbf{S T}$.

Proof The natural embedding $\mathfrak{k}_{U}$ is the required isomorphism. Every realization satisfies $\mathbf{Z F C}{ }^{\text {st }}$. For every $f \in \mathbb{V}^{U}$ with $\operatorname{ran} f \subseteq A, f \in_{U} \mathfrak{k}_{U}(A)$. Transfer is provided by Łoś Theorem. Every bounded realization trivially satisfies Inner Standardization.

Until further notice, we work in the elementary nonstandard set theory $\mathbf{S T}^{-}$.

If $\mathbb{U}$ is a class, $\mathcal{P}^{\mathbb{U}}$ is a formula obtained from $\mathcal{P}$ by replacing each occurrence of $(\exists x) \ldots[(\forall x) \ldots$, resp.] by $(\exists x \in \mathbb{U}) \ldots[(\forall x \in \mathbb{U}) \ldots$, resp.]. In particular, $\mathcal{P}^{\mathbb{S}}\left(x_{1}, \ldots, x_{k}\right) \Leftrightarrow \mathcal{P}^{\text {st }}\left(x_{1}, \ldots, x_{k}\right)$ and $\mathcal{P}^{\mathbb{V}}\left(x_{1}, \ldots, x_{k}\right) \Leftrightarrow \mathcal{P}\left(x_{1}, \ldots, x_{k}\right)$. We use $\mathbb{U}_{1} \preccurlyeq$ $\mathbb{U}_{2}\left(\mathbb{U}_{2}\right.$ is an $\in$-elementary extension of $\left.\mathbb{U}_{1}\right)$ as shorthand for the assertion that, for any $\in$-formula $\mathcal{P}\left(x_{1}, \ldots, x_{k}\right), \quad\left(\forall x_{1}, \ldots, x_{k} \in \mathbb{U}_{1}\right)\left(\mathcal{P}^{\mathbb{U}_{1}}\left(x_{1}, \ldots, x_{k}\right) \Leftrightarrow \mathcal{P}^{\mathbb{U}_{2}}\left(x_{1}, \ldots, x_{k}\right)\right)$. In particular, Transfer is just the assertion that $\mathbb{S} \preccurlyeq \mathbb{V}$.

Proposition 3.2 $\left(\mathbf{S T}^{-}\right.$) Let $\mathbb{S} \subseteq \mathbb{U} \subseteq \mathbb{V}$. The following statements are equivalent:

$$
\begin{gathered}
\mathbb{S} \preccurlyeq \mathbb{U} \\
\mathbb{U} \preccurlyeq \mathbb{V} \\
(\forall x, y \in \mathbb{U})[(x, y) \in \mathbb{U} \wedge(\forall x \in \mathbb{U})(\forall f \in \mathbb{S})(f(x) \in \mathbb{U})] .
\end{gathered}
$$


Proof $(1) \Rightarrow(3)$ :

ZFC $^{\text {st }}$ holds, so $(\forall x, y \in \mathbb{S})[(x, y) \in \mathbb{S} \wedge(\forall x \in \mathbb{S})(\forall f \in \mathbb{S})(f(x) \in \mathbb{S})]$. From (1) we get that this statement holds with $\mathbb{S}$ replaced everywhere by $\mathbb{U}$.

$(2) \Rightarrow(1)$ :

Let $\mathcal{P}(\bar{x})$ be an $\in$-formula, $\bar{x} \in \mathbb{S}$. If $\mathcal{P}^{\mathbb{S}}(\bar{x})$ holds, then $\mathcal{P}(\bar{x})$ holds by $\mathbb{S} \preccurlyeq \mathbb{V}$, and then $\mathcal{P}^{\mathbb{U}}(\bar{x})$ holds by $\mathbb{U} \preccurlyeq \mathbb{V}$ and $\mathbb{S} \subseteq \mathbb{U}$.

(3) $\Rightarrow(2)$ :

Claim. $\left(\forall x_{1}, \ldots, x_{k} \in \mathbb{U}\right)\left((\exists y) \mathcal{P}\left(x_{1}, \ldots, x_{k}, y\right) \Rightarrow(\exists y \in \mathbb{U}) \mathcal{P}\left(x_{1}, \ldots, x_{k}, y\right)\right)$.

Proof Assume $x_{1}, \ldots, x_{k} \in \mathbb{U}$ and $(\exists y) \mathcal{P}(\bar{x}, y)$. By repeated application of (3) we know that $\left(x_{1}, \ldots, x_{k}\right) \in \mathbb{U}$, and by Boundedness we obtain $A \in \mathbb{S}$ such that $\left(x_{1}, \ldots, x_{k}\right) \in A$. Axioms of Separation hold in $\mathbb{S}$, so there is a set $C \in \mathbb{S}$ such that $\left(\forall^{\text {st }} \bar{z}\right)\left[\left(z_{1}, \ldots, z_{k}\right) \in C \Leftrightarrow\left(z_{1}, \ldots, z_{k}\right) \in A \wedge\left(\exists^{\text {st }} y\right) \mathcal{P}^{\text {st }}(\bar{z}, y)\right]$. By Transfer then also $(\forall \bar{z})\left[\left(z_{1}, \ldots, z_{k}\right) \in C \Leftrightarrow\left(z_{1}, \ldots, z_{k}\right) \in A \wedge(\exists y) \mathcal{P}(\bar{z}, y)\right]$, so in particular $\left(x_{1}, \ldots, x_{k}\right) \in C$. Axioms of Selection hold in $\mathbb{S}$, so there is a function $f \in \mathbb{S}$ such that $\left(\forall^{\text {st }} \bar{z} \in C\right) \mathcal{P}^{\text {st }}(\bar{z}, f(\bar{z}))$. Again by Transfer, $(\forall \bar{z} \in C) \mathcal{P}(\bar{z}, f(\bar{z}))$, and in particular $\mathcal{P}(\bar{x}, f(\bar{x}))$ holds. But $f(\bar{x}) \in \mathbb{U}$ by $(3)$.

The proof that $\mathbb{U} \preccurlyeq \mathbb{V}$ now proceeds by induction on complexity of $\mathcal{P}$. The only nontrivial case is when $\mathcal{P}(\bar{x})$ is of the form $(\exists y) \mathcal{Q}(\bar{x}, y)$. We assume by induction that $(\forall \bar{x}, y \in \mathbb{U})\left(\mathcal{Q}^{\mathbb{U}}(\bar{x}, y) \Leftrightarrow \mathcal{Q}(\bar{x}, y)\right)$ holds. Using the Claim in the second step and the inductive assumption in the third one, we have, for $\bar{x} \in \mathbb{U}, \mathcal{P}(\bar{x}) \Leftrightarrow(\exists y) \mathcal{Q}(\bar{x}, y) \Leftrightarrow$ $(\exists y \in \mathbb{U}) \mathcal{Q}(\bar{x}, y) \Leftrightarrow(\exists y \in \mathbb{U}) \mathcal{Q}^{\mathbb{U}}(\bar{x}, y) \Leftrightarrow \mathcal{P}^{\mathbb{U}}(\bar{x})$.

Definition 3.3 $\mathbb{S}[a]:=\{f(a): f \in \mathbb{S}$ is a function $\}$.

\section{Corollary $3.4\left(\mathrm{ST}^{-}\right)$}

$\mathbb{S} \preccurlyeq \mathbb{S}[a] \preccurlyeq \mathbb{V}$, and if $\mathbb{S} \preccurlyeq \mathbb{U}$ and $a \in \mathbb{U}$, then $\mathbb{S}[a] \preccurlyeq \mathbb{U}$.

Proof If $x=f(a)$ and $y=g(a)$ for $f, g \in \mathbb{S}$, then $(x, y)=F(a)$ for $F \in \mathbb{S}$ defined by $F(z)=(f(z), g(z))$. Hence $\mathbb{S}[a]$ satisfies (3) of Proposition 3.2.

$\mathbb{S}[a]$ is the least $\in$-elementary extension of $\mathbb{S}$ containing $a$ (as an element). It turns out that $\mathbb{S}[a]$ is naturally isomorphic to an ultrapower of the standard universe $\mathbb{S}$, constructed inside $\mathbb{S}$ (Proposition 3.7).

Let $a \in A \in \mathbb{S}$ and let $W_{a, A}:=\{X \subseteq A: a \in X\}$ be the principal ultrafilter over $A$ containing $\{a\}$. If the standard set $V_{a, A}:=\mathbf{s h}\left(W_{a, A}\right)$ exists, then it has the property 
that $X \in V_{a, A} \Leftrightarrow a \in X$, for $X \in \mathcal{P}(A) \cap \mathbb{S}$. It is trivial to verify that $V_{a, A}$ is a (standard) ultrafilter over $A$, and that $V_{a, A}$ is principal iff $a \in \mathbb{S}$ (iff $V_{a, A}=W_{a, A}$ ). We say that $a$ generates the standard ultrafilter $V_{a, A}$ over $A$. We also write $a \mathbb{M} U$ ( $a$ is in the monad of $U)$ for $\left(\forall^{\text {st }} X \in \mathcal{P}(A)\right)(X \in U \Leftrightarrow a \in X)$. Clearly, if $a \in A \cap B$ where $A, B \in \mathbb{S}$, then $V_{a, A} \sim V_{a, B}$.

Proposition 3.5 $\left(\mathbf{S T}^{-}\right)$Assume $A, B, f \in \mathbb{S}, f: \subseteq A \rightarrow B, a \in \operatorname{dom} f$ and $V_{a, A}$ exists. Then $V_{f(a), B}=f\left[V_{a, A}\right]$.

Proof The set $f\left[V_{a, A}\right] \in \mathbb{S}$ and, for $Y \in \mathcal{P}(B) \cap \mathbb{S}, \quad Y \in f\left[V_{a, A}\right] \Leftrightarrow f^{-1}[Y] \in V_{a, A} \Leftrightarrow$ $a \in f^{-1}[Y] \Leftrightarrow f(a) \in Y$.

Proposition 3.6 $\left(\mathbf{S T}^{-}\right)$The following statements are equivalent:

(a) Inner Standardization

(b) For every $a \in A \in \mathbb{S}$ there exists the ultrafilter $V_{a, A} \in \mathbb{S}$ generated by a over $A$.

Proof The proof of (a) $\Rightarrow$ (b) is contained in the discussion above. For (b) $\Rightarrow$ (a) it suffices to show that $\mathbf{s h}(a)$ is $\in$-definable from $V_{a, A}$.

Let $a \in A \in \mathbb{S}$; then $a \subseteq \bigcup A$. For each $y \in \bigcup A$ let $X_{y}:=\{x \in A: y \in x\} \subseteq A$; we note that $\left\langle X_{y}: y \in \bigcup A\right\rangle$ is a standard function; hence $b:=\left\{y \in \bigcup A: X_{y} \in V_{a, A}\right\} \in \mathbb{S}$. For $y \in \bigcup A \cap \mathbb{S}$ we have $y \in a \Leftrightarrow a \in X_{y} \Leftrightarrow X_{y} \in V_{a, A}$, so $\left(\forall^{\text {st }} y\right)(y \in b \Leftrightarrow y \in a)$, and $b=\mathbf{s h}(a)$.

We continue working in $\mathbf{S T}^{-}$; let $a \in A \in \mathbb{S}$ be such that $U:=V_{a, A}$ exists. $\mathcal{S}:=$ $(\mathbb{S},=, \in)$ is an interpretation of $\mathbf{Z F C}$ and $\mathcal{S} \preccurlyeq(\mathbb{V},=, \in)$; we consider the ultrapower of the universe modulo $U$ constructed inside $\mathcal{S}$ :

$$
\begin{aligned}
& {[\mathcal{U} \ell t(\mathbb{V} ; U)]^{\mathcal{S}}=\left(\left(\mathbb{V}^{U}\right)^{\mathcal{S}},\left(==_{U}\right)^{\mathcal{S}},\left(\in_{U}\right)^{\mathcal{S}},\left(\mathbb{S}_{U}\right)^{\mathcal{S}}\right)=} \\
& \left(\mathbb{V}^{U} \cap \mathbb{S},={ }_{U} \cap(\mathbb{S} \times \mathbb{S}), \in_{U} \cap(\mathbb{S} \times \mathbb{S}), \mathbb{S}_{U} \cap \mathbb{S}\right)
\end{aligned}
$$

(the second equality follows by Transfer; see also Section 1).

We define a mapping $\mathfrak{j}_{a, A}: \mathbb{V}^{U} \cap \mathbb{S} \rightarrow \mathbb{V}$ by $\mathfrak{j}_{a, A}(f)=f(a)$.

Proposition $3.7\left(\mathbf{S T}^{-}\right) \quad \mathfrak{j}_{a, A}$ is an isomorphism between $\left[\mathcal{U} \ell t\left(\mathbb{V} ; V_{a, A}\right)\right]^{\mathcal{S}}$ and $\mathcal{S}_{a}:=$ $(\mathbb{S}[a],=, \in, \mathbb{S})$, and $\mathfrak{j}_{a, A}\left(I d_{A}\right)=a$.

Proof Let $\mathfrak{j}:=\mathfrak{j}_{a, A}$. Clearly $\operatorname{ran} \mathfrak{j}=\{f(a): f \in \mathbb{S}\}=\mathbb{S}[a]$. For standard $f, g$ we have $f={ }_{U} g \Leftrightarrow\{i \in A: f(i)=g(i)\} \in U \Leftrightarrow a \in\{i \in A: f(i)=g(i)\} \Leftrightarrow f(a)=$ $g(a) \Leftrightarrow \mathrm{j}(f)=\mathrm{j}(g)$. Similarly $f \in_{U} g \Leftrightarrow \mathrm{j}(f) \in \mathfrak{j}(g)$. For $x \in \mathbb{S}, \mathfrak{j}\left(\mathfrak{k}_{U}(x)\right)=x$, so $\mathfrak{j}$ maps $\mathbb{S}_{U} \cap \mathbb{S}$ onto $\mathbb{S}$. Clearly $\mathfrak{j}\left(I_{A}\right)=I d_{A}(a)=a$. 
Let $\mathbf{S T}^{p}$ be $\mathbf{S T}$ plus

Axiom of Primitivity: $\quad(\exists a)(\forall x)\left(\exists^{\text {st }} f\right)(x=f(a))$.

Corollary 3.8 $\left(\mathbf{S T}^{p}\right)$ The trivial interpretation $(\mathbb{V},=, \in, \mathbb{S})$ is isomorphic to an ultrapower of the universe constructed inside $\mathcal{S}$.

There is a useful interpretation of this result in $\mathbf{Z F C}$.

Corollary 3.9 (ZFC) Ultrapowers of $\mathbb{V}$ are precisely the realizations of $\mathbf{S T}^{p}$.

Proof $\mathcal{U} \ell t(\mathbb{V} ; U)$ is a realization of $\mathbf{S T}$ by Proposition 3.1. To prove that primitivity holds, take $a=I d_{I}$.

If $\mathfrak{M}$ is a realization of $\mathbf{S T}^{p}$, Corollary 3.8 is true in $\mathfrak{M}$ and, by remarks following Proposition 1.4, $\mathfrak{M}$ is isomorphic to $\left[[\mathcal{U} \ell t(\mathbb{V} ; U)]^{\mathcal{S}}\right]^{\mathfrak{M}}=[\mathcal{U} \ell t(\mathbb{V} ; U)]^{\mathcal{S}_{\mathfrak{M}}}$. But $\mathcal{S}_{\mathfrak{M}}$ is isomorphic to $\mathcal{V}$ via $\mathfrak{k}$, so $[\mathcal{U} \ell t(\mathbb{V} ; U)]^{\mathcal{S}_{\mathfrak{M}}}$ is isomorphic to $\mathcal{U} \ell t\left(\mathbb{V} ; \mathfrak{k}^{-1}(U)\right)$.

In this sense the nonstandard set theory $\mathbf{S T}^{p}$ axiomatizes ultrapowers of the universe. Here are some further observations related to these ideas.

Lemma 3.10 ( ZFC) (a) $\mathcal{U} \ell t(\mathbb{V} ; U) \vDash h=\mathfrak{k}_{U}(g)(f)$ if and only if $h={ }_{U} g \circ f$.

Assume that $f \in_{U} \mathfrak{k}_{U}(A)$.

(b) $\mathcal{U} \ell t(\mathbb{V} ; U) \vDash V_{f, \mathfrak{k}_{U}(A)}=\mathfrak{k}_{U}(f[U])$.

(c) $f \in \mathbb{S}_{U}$ if and only if $\mathcal{U} \ell t(\mathbb{V} ; U) \vDash " V_{f, \mathfrak{k}_{U}(A)}$ is principal".

Proof We write $\mathfrak{k}$ for $\mathfrak{k}_{U}$.

(a) $\mathcal{U} \ell t(\mathbb{V} ; U) \vDash h=\mathfrak{k}(g)(f)$ iff $\{i: h(i)=(\mathfrak{k}(g)(i))(f(i))\} \in U$ iff $\{i: h(i)=$ $g(f(i))\} \in U$ iff $h={ }_{U} g \circ f$.

(b) For $X \subseteq A, \mathcal{U} \ell t(\mathbb{V} ; U) \vDash \mathfrak{k}(X) \in V_{f, \mathfrak{k}(A)}$ iff $f \in_{U} \mathfrak{k}(X)$ iff $\{i: f(i) \in X\} \in U$ iff $f^{-1}[X] \in U$ iff $X \in f[U]$ iff $\mathfrak{k}(X) \in_{U} \mathfrak{k}(f[U])$.

(c) Using (b), $\mathcal{U} \ell t(\mathbb{V} ; U) \vDash$ " $V_{f, \mathfrak{k}(A)}$ is principal" iff $f[U]$ is principal. But $\{a\} \in f[U]$ for some $a \in A$ iff $f={ }_{U} \mathfrak{k}(a)$ for some $a \in A$, ie, iff $f \in \mathbb{S}_{U}$.

We return to the theory ST. 
Proposition 3.11 (ST) If $a \in \mathbb{S}$ is finite, then $a \subseteq \mathbb{S}$.

Proof We first show that $(\forall n \in \omega)(n \in \mathbb{S} \Rightarrow n \subseteq \mathbb{S})$. Assume $m \in n$ is nonstandard. By Inner Standardization, there is $y \in \mathbb{S}$ such that $\left(\forall^{\mathbf{s t}} z\right)(z \in y \Leftrightarrow z \in m)$. By Transfer, $y \subseteq \omega, y$ is nonempty $(0 \in y)$ and bounded above (by $n)$; let $m_{0}$ be the greatest element of $y$. As $m_{0}$ is standard, $m_{0} \in m$; so either $m_{0}+1 \in m$ and $m_{0}+1 \in y$, or $m_{0}+1=m$ and $m$ is standard. Either way, we have a contradiction.

If now $a \in \mathbb{S}$ is finite, there is a one-one mapping $f \in \mathbb{S}$ of some $n \in \mathbb{S}$ onto $a$. Then $n \subseteq \mathbb{S}$ and hence $a=f[n] \subseteq \mathbb{S}$, by Transfer.

Let $\kappa$ be a standard infinite cardinal.

$\kappa$-Idealization is the statement:

For all standard $A, B$ with $|A|<\kappa$ and all $R \subseteq A \times B$,

$$
\left(\forall^{\text {st }} a \in \mathcal{P}^{\text {fin }} A\right)(\exists y)(\forall x \in a)(\langle x, y\rangle \in R) \Leftrightarrow(\exists y)\left(\forall^{\mathbf{s t}} x \in A\right)(\langle x, y\rangle \in R) .
$$

It is well-known (see Chang-Keisler [6]) that if the ultrafilter $U$ is $\kappa$-good ${ }^{3}$, then $\mathcal{U} \ell t(\mathbb{V} ; U)$ is $\kappa$-saturated, that is, in our terminology, $\mathcal{U} \ell t(\mathbb{V} ; U) \vDash \kappa$-Idealization. [More accurately, we should say $\mathcal{U} \ell t(\mathbb{V} ; U) \vDash \mathfrak{k}_{U}(\kappa)$-Idealization, etc.]

Proposition 3.12 (ST $+\kappa$-Idealization) Let $U, V, f \in \mathbb{S}, U=f[V]$ and $a \mathbb{M} U$. If $|V|<\kappa$, then there exists $b \mathbb{M} V$ such that $f(b)=a$.

Proof Let $A:=V, B:=\operatorname{dom} V,\langle X, y\rangle \in R \Leftrightarrow y \in X \wedge f(y)=a$. If $S \subseteq V$ is standard finite, then $\bigcap S=X_{0}$ for some $X_{0} \in V \cap \mathbb{S}$. Hence $f\left[X_{0}\right] \in U \cap \mathbb{S}$ and so $a \in f\left[X_{0}\right]$, ie, $a=f(y)$ for some $y \in X_{0}$. It follows that $\langle X, y\rangle \in R$ for all $X \in S \cap \mathbb{S}$. By $\kappa$-Idealization there exists $b$ such that $\langle X, b\rangle \in R$ for all $X \in V \cap \mathbb{S}$, ie, $b \mathbb{M} V$ and $f(b)=a$.

The special case where $U$ is principal over $\{0\}$ is also useful; the assertion then is that, if $|V|<\kappa$, then there is $b \mathbb{M} V$.

Proposition 3.13 (ZFC) (Amalgamation Lemma) Let $U, V, W$ be ultrafilters, $V=$ $f[U], V=g[W]$. If $\widehat{U}$ is a $\kappa$-good ultrafilter and $\kappa>|U|,|W|$, then there exist $\widehat{f}$ such that $\widehat{f}[\widehat{U}]=U$. For each such $\widehat{f}$ there exists $\widehat{g}$ such that $\widehat{g}[\widehat{U}]=W$ and $f \circ \widehat{f}=\widehat{U} g \circ \widehat{g}$. That is, the following diagram commutes.

\footnotetext{
${ }^{3}$ In this paper, all $\kappa$-good ultrafilters are assumed to be $\omega_{1}$-incomplete.
} 


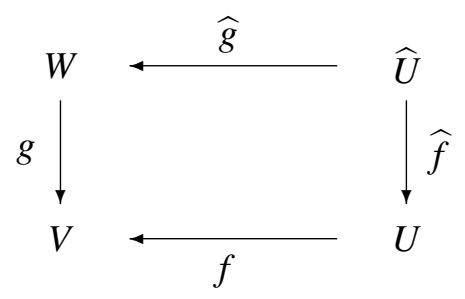

Proof We write $\mathfrak{k}$ for $\mathfrak{k}_{\widehat{U}}$. $\mathcal{U} \ell t(\mathbb{V} ; \widehat{U}) \vDash$ "k $(\kappa)$-Idealization", so Proposition 3.12 holds in $\mathcal{U} \ell t(\mathbb{V} ; \widehat{U})$. When applied to $\mathfrak{k}(U)$, this implies that there is $\widehat{f} \in \mathbb{V}^{\widehat{U}}$ such that $\mathcal{U} \ell t(\mathbb{V} ; \widehat{U}) \vDash \widehat{f} \mathbb{M} \mathfrak{k}(U)$; that is, $\widehat{f} \in \widehat{U} \mathfrak{k}(A)$ for all $A \in U$, ie, $\widehat{f}^{-1}[A] \in \widehat{U}$ for all $A \in U$. We then have $U=\widehat{f}[\widehat{U}]$.

Given $\widehat{f}$ such that $\widehat{f}[\widehat{U}]=U$, we have $V=h[\widehat{U}]$ where $h:=f \circ \widehat{f}$. Hence $h \in_{\widehat{U}} \mathfrak{k}(B)$, for every $B \in V$, ie, $\mathcal{U} \ell t(\mathbb{V} ; \widehat{U}) \vDash h \mathbb{M k}(V)$. Also $\mathcal{U} \ell t(\mathbb{V} ; \widehat{U}) \vDash \mathfrak{k}(V)=\mathfrak{k}(g)[\mathfrak{k}(W)]$ and $\mathcal{U} \ell t(\mathbb{V} ; \widehat{U}) \vDash|\mathfrak{k}(W)|<\mathfrak{k}(\kappa)$. Applying Proposition 3.12 inside $\mathcal{U} \ell t(\mathbb{V} ; \widehat{U})$ once more, we obtain $\widehat{g} \in \mathbb{V}^{\widehat{U}}$ such that $\mathcal{U} \ell t(\mathbb{V} ; \widehat{U}) \vDash " \widehat{g} \mathbb{M k}(W) \wedge h=\mathfrak{k}(g)(\widehat{g})$ ". This translates into $W=\widehat{g}[\widehat{U}]$ and $h=\widehat{U} g \circ \widehat{g}$.

\section{Repeated Ultrapowers.}

Let $\mathcal{U} \ell(\mathbb{V} ; U) \vDash$ " $u$ is an ultrafilter"; we can then form the ultrapower of the universe modulo $u$ inside $\mathcal{U} \ell(\mathbb{V} ; U)$, ie, consider the interpretation

$$
[\mathcal{U} \ell t(\mathbb{V} ; u)]^{\mathcal{U} \ell(\mathbb{V} ; U)}=\left(\left(\mathbb{V}^{u}\right)^{\mathcal{U} \ell(\mathbb{V} ; U)},\left(={ }_{u}\right)^{\mathcal{U} \ell(\mathbb{V} ; U)},\left(\in_{u}\right)^{\mathcal{U} \ell(\mathbb{V} ; U)},\left(\mathbb{S}_{u}\right)^{\mathcal{U} \ell(\mathbb{V} ; U)}\right) .
$$

We observe that $u=\left\langle U_{i}: i \in \operatorname{dom} u\right\rangle$ is a function with $\operatorname{dom} u \in U$, and for $U$-almost all $i, U_{i}$ is an ultrafilter; without loss of generality we can assume that $\operatorname{dom} u=I$ and $U_{i}$ is an ultrafilter for all $i \in I$. Now $F \in\left(\mathbb{V}^{u}\right)^{\mathcal{U} \ell(\mathbb{V} ; U)}$ iff $\mathcal{U} \ell(\mathbb{V} ; U) \vDash " F$ is a function $\wedge \operatorname{dom} F \in u$ " iff $F$ is a function, $\operatorname{dom} F \in U$ and $\left\{i \in I: F(i) \in \mathbb{V}^{U_{i}}\right\} \in U$ iff $F \in \prod \mathbb{V}^{U_{i}} / U$. For $F, G \in\left(\mathbb{V}^{u}\right)^{\mathcal{U} \ell(\mathbb{V} ; U)}, \mathcal{U} \ell(\mathbb{V} ; U) \vDash F={ }_{u} G$ iff $\{i \in I$ : $\left.F(i)={ }_{U_{i}} G(i)\right\} \in U$ iff $\langle F, G\rangle \in \prod\left(==_{U_{i}}\right) / U$. Similarly for $\in_{u} . F \in\left(\mathbb{S}_{u}\right)^{\mathcal{M}(\mathbb{V} ; U)}$ iff $\left\{i \in I: F(i) \in \mathbb{S}_{U_{i}}\right\} \in U$ iff $F \in \prod \mathbb{S}_{U_{i}} / U$.

It follows from these observations that the interpretation $[\mathcal{U} \ell t(\mathbb{V} ; u)]^{\mathcal{U} \ell(\mathbb{V} ; U)}$ is nothing but the ultraproduct $\mathcal{U} \ell\left(\left\langle\mathcal{U} \ell t\left(\mathbb{V} ; U_{i}\right): i \in I\right\rangle ; U\right)$.

Let $U$ be an ultrafilter over $I$ and $\left\langle U_{i}: i \in I\right\rangle$ a sequence of ultrafilters, with $U_{i}$ over $I_{i}$. Let $K:=\Sigma_{i \in I} I_{i}:=\bigcup_{i \in I}\{i\} \times I_{i}=\left\{\langle i, j\rangle: i \in I, j \in I_{i}\right\}$.

The Rudin-Frolík sum $\Sigma_{U} U_{i}$ is the ultrafilter $V$ over $K$ defined as follows:

$$
X \in V \Leftrightarrow\left\{i \in I:(X)_{i} \in U_{i}\right\} \in U \Leftrightarrow\left\{i \in I:\left\{j \in I_{i}:\langle i, j\rangle \in X\right\} \in U_{i}\right\} \in U .
$$


The verification that $V$ is indeed an ultrafilter over $K$ and that $\pi_{1}[V]=U$ is trivial.

In the special case where $U_{i}=U^{\prime}$ for all $i \in I$, we write $U \otimes U^{\prime}$ (product of $U$ and $\left.U^{\prime}\right)$ in place of $\Sigma_{U} U_{i} ; U \otimes U^{\prime}$ is an ultrafilter over $I \times I^{\prime}$, for $I^{\prime}:=\operatorname{dom} U^{\prime}$.

The relation $\leq_{R F}$ on ultrafilters, defined by $U \leq_{R F} V$ iff $V$ is isomorphic to $\Sigma_{U} U_{i}$ for some $\left\langle U_{i}: i \in I\right\rangle$, is called the Rudin-Frolík preordering. Transitivity of $\leq_{R F}$ follows from the evident associativity of Rudin-Frolík sums: Given ultrafilters $V_{i, j},\langle i, j\rangle \in K$, and $V=\Sigma_{U} U_{i}, W=\Sigma_{V} V_{i, j}$, we have also $W \cong \Sigma_{U} W_{i}$ where $W_{i}=\Sigma_{U_{i}} V_{i, j}$. Clearly $U \leq_{R F} V$ implies $U \leq_{R K} V$ (via $\left.U=\pi_{1}[V]\right)$.

Let $f \in \mathbb{V}^{V}$; we define the function $f / I \in \mathbb{V}^{U}$ by $(f / I)(i)=(f)_{i}$, where $\operatorname{dom}(f)_{i} \subseteq I_{i}$ and $(f)_{i}(j)=f(i, j)$. Also $\Omega(f):=f / I$.

Proposition 4.1 (The Factoring Lemma) $\Omega$ is an isomorphism of $\mathcal{U} \ell\left(\mathbb{V} ; \Sigma_{U} U_{i}\right)$ and $\mathcal{U} \ell\left(\left\langle\mathcal{U} \ell\left(\mathbb{V} ; U_{i}\right): i \in I\right\rangle ; U\right)=[\mathcal{U} \ell(\mathbb{V} ; u)]^{\mathcal{U} \ell(\mathbb{V} ; U)}$.

Moreover, for $h \in \mathbb{V}^{U}, \mathcal{U} \ell(\mathbb{V} ; U) \vDash \Omega\left(\pi_{1}^{*}(h)\right)={ }_{u} \mathfrak{k}_{u}(h)$.

Here $\mathfrak{k}_{u}$ is the natural embedding of the universe into its ultrapower modulo $u$, all evaluated inside of $\mathcal{U} \ell(\mathbb{V} ; U)$.

An equivalent formulation of the Factoring Lemma is:

$\Omega$ is an isomorphism of $\left(\mathcal{U} \ell\left(\mathbb{V} ; \Sigma_{U} U_{i}\right), \pi_{1}^{*}\left[\mathbb{V}^{U}\right]\right)$ and $\mathcal{U} \ell\left(\left\langle\mathcal{U} \ell t\left(\mathbb{V} ; U_{i}\right): i \in I\right\rangle ; U\right)=$ $[\mathcal{U} \ell t(\mathbb{V} ; u)]^{\mathcal{U} \ell(\mathbb{V} ; U)}$.

Proof We first note that, for $f \in \mathbb{V}^{V}$, the set $\left\{i \in I: \operatorname{dom}((f / I)(i)) \in U_{i}\right\} \in U$, so $\Omega(f) \in \prod \mathbb{V}^{U_{i}} / U$.

We have $f_{1}={ }_{V} f_{2} \quad \Leftrightarrow \quad\left\{\langle i, j\rangle \in K: f_{1}(i, j)=f_{2}(i, j)\right\} \in \Sigma_{U} U_{i} \quad \Leftrightarrow$ $\left\{i \in I:\left\{j \in I_{i}:\left(f_{1} / I\right)(i)(j)=\left(f_{2} / I\right)(i)(j)\right\} \in U_{i}\right\} \in U \quad \Leftrightarrow$

$\left\{i \in I:\left(f_{1} / I\right)(i)={ }_{U_{i}}\left(f_{2} / I\right)(i)\right\} \in U \quad \Leftrightarrow \mathcal{U} \ell(\mathbb{V} ; U) \vDash \Omega\left(f_{1}\right)={ }_{u} \Omega\left(f_{2}\right)$.

A similar calculation shows that $\Omega$ preserves $\epsilon$.

To show that $\Omega$ is "onto", we observe that $g \in \prod \mathbb{V}^{U_{i}} / U$ implies that $U$-almost everywhere $g(i)$ is a function with $\operatorname{dom} g(i) \in U_{i}$. We let $f(i, j):=g(i)(j)$ where defined; $\operatorname{dom} f \in V$ and $\left\{i \in I:(f / I)(i)={ }_{U_{i}} g(i)\right\} \in U$, so $\mathcal{U} \ell(\mathbb{V} ; U) \vDash \Omega(f)={ }_{u} g$.

For $h \in \mathbb{V}^{U}, \pi_{1}^{*}(h)(i, j)=h(i)$ for all $i \in \operatorname{dom} h \in U, j \in I_{i}$. Hence $\Omega\left(\pi_{1}^{*}(h)\right)=$ $\left\langle\mathfrak{k}_{U_{i}}(h(i)): i \in \operatorname{dom} h\right\rangle$ where $\mathfrak{k}_{U_{i}}(h(i))$ is the constant function on $I_{i}$ with value $h(i)$. This implies that $\mathcal{U} \ell(\mathbb{V} ; U) \vDash \Omega\left(\pi_{1}^{*}(h)\right)={ }_{u} \mathfrak{k}_{u}(h)$.

Proposition 4.1 gives an easy proof of an important fact. 
Proposition If $U \leq_{R F} V$ and $U$ is $\kappa$-good, then $V$ is $\kappa$-good.

Proof According to Chang-Keisler [6], the ultrafilter $V$ over the set $K$ is $\kappa$-good if and only if $\mathcal{U} \ell\left(\left\langle\mathfrak{A}_{i, j}:\langle i, j\rangle \in K\right\rangle ; V\right)$ is $\kappa$-saturated, for all structures $\mathfrak{A}_{i, j}$ in any language with less than $\kappa$ symbols. By the Factoring Lemma, this ultraproduct is isomorphic to $\mathcal{U} \ell\left(\left\langle\mathcal{U} \ell\left(\left\langle\mathfrak{A}_{i, j}: j \in I_{i}\right\rangle ; U_{i}\right): i \in I\right\rangle ; U\right)$, which is $\kappa$-saturated when $U$ is $\kappa$-good.

The interpretation $\mathcal{U} \ell(\mathbb{V} ; U)$ satisfies $\mathbf{Z F C}$; consequently, Corollary 3.9 holds in

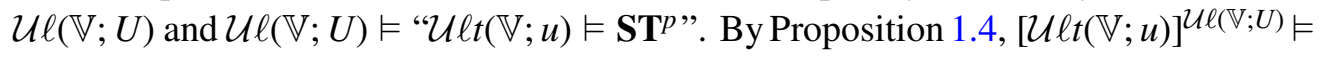
$\mathbf{S T}^{p}$ and, in view of the isomorphism provided by the Factoring Lemma, we have $\left(\mathbb{V}^{V},=_{V}, \in_{V}, \pi_{1}^{*}\left[\mathbb{V}^{U}\right]\right) \vDash \mathbf{S T}^{p}$. On the other hand, as for any ultrapower, we have also $\left(\mathbb{V}^{V},=_{V}, \in_{V}, \mathbb{S}_{V}\right) \vDash \mathbf{S T}^{p}$, where $\mathbb{S}_{V}=\mathfrak{k}_{V}[\mathbb{V}]$.

The point is that the repeated ultrapower has two candidates for a "standard universe". We consider the interpretation $\left(\mathbb{V}^{V},=_{V}, \in_{V}, \mathfrak{k}_{V}[\mathbb{V}], \pi_{1}^{*}\left[\mathbb{V}^{U}\right]\right)$ for the language with $\in$ and two unary predicates, $\mathbf{s t}_{0}$ and $\mathbf{s t}_{1}$, with $\mathbf{s t}_{0}(x)$ interpreted by " $x \in \mathfrak{k}_{V}[\mathbb{V}]$ " and $\mathbf{s t}_{1}$ by " $x \in \pi_{1}^{*}\left[\mathbb{V}^{U}\right]$ ". This interpretation satisfies $\mathbf{S T}_{0} \wedge \mathbf{S} \mathbf{T}_{1}$, where $\mathbf{S T}_{0}$ and $\mathbf{S} \mathbf{T}_{1}$ are obtained from $\mathbf{S T}$ by replacing $\mathbf{s t}$ with $\mathbf{s t}_{0}$ and $\mathbf{s t}_{1}$, respectively.

We now work in $\mathbf{S T}_{0} \wedge \mathbf{S T}_{1}$ and let $\mathbb{S}_{0}:=\left\{x: \mathbf{s t}_{0}(x)\right\}, \mathbb{S}_{1}:=\left\{x: \mathbf{s t}_{1}(x)\right\}$. For $a \in A \in \mathbb{S}_{0}$ there is the ultrafilter $V_{a, A}^{0} \in \mathbb{S}_{0}$ such that

$$
\left(\forall X \in \mathcal{P}(A) \cap \mathbb{S}_{0}\right)\left(X \in V_{a, A}^{0} \Leftrightarrow a \in X\right),
$$

and the ultrafilter $V_{a, A}^{1} \in \mathbb{S}_{1}$ such that

$$
\left(\forall X \in \mathcal{P}(A) \cap \mathbb{S}_{1}\right)\left(X \in V_{a, A}^{1} \Leftrightarrow a \in X\right) .
$$

We have $a \in \mathbb{S}_{0}$ iff $V_{a, A}^{0}$ is principal, as well as $a \in \mathbb{S}_{1}$ iff $V_{a, A}^{1}$ is principal. Also, $\left[\mathcal{U} \ell t\left(\mathbb{V} ; V_{a, A}^{0}\right)\right]^{\mathcal{S}_{0}}$ is isomorphic to $\left(\mathbb{S}_{0}[a],=, \in, \mathbb{S}_{0}\right)$, the least $\in$-elementary extension of $\mathbb{S}_{0}$ containing $a$, and $\left[\mathcal{U} \ell t\left(\mathbb{V} ; V_{a, A}^{1}\right)\right]^{\mathcal{S}_{1}}$ is isomorphic to $\left(\mathbb{S}_{1}[a],=, \in, \mathbb{S}_{1}\right)$, the least $\in$-elementary extension of $\mathbb{S}_{1}$ containing $a$. Trivially, $\mathbb{S}_{0}[a]$ is closed under $\mathbf{s h}_{0}$, and $\mathbb{S}_{1}[a]$ is closed under both $\mathbf{s h}_{0}$ and $\mathbf{s h}_{1}$; but we need a finer result.

Proposition $4.2\left(\mathbf{S T}_{0} \wedge \mathbf{S T}_{1}\right) \quad \mathbb{S}_{0}[[a]]:=\mathbb{S}_{0}\left[\left\langle V_{a, A}^{1}, a\right\rangle\right]$ is the least $\in$-elementary extension of $\mathbb{S}_{0}$ containing $a$ and closed under $\mathbf{s h}_{0}$ and $\mathbf{s} \mathbf{h}_{1}$.

In particular, it is independent of the choice of $A$. However, it is crucial that $A \in \mathbb{S}_{0}$, although the notion of $V_{a, A}^{1}$ is defined for any $a \in A \in \mathbb{S}_{1}$. 
Proof Trivially, $\mathbb{S}_{0}[[a]] \supseteq \mathbb{S}_{0}$ is closed under $\mathbf{s h}_{0}$. We prove that it is also closed under $\mathbf{s h}_{1}$.

Let $\boldsymbol{\beta} A$ be the set of all ultrafilters over $A$, and $V^{1}:=V_{a, A}^{1} ;\left\langle V^{1}, a\right\rangle \in \boldsymbol{\beta} A \times A \in \mathbb{S}_{0}$. For $X \in \mathcal{P}(\boldsymbol{\beta} A \times A) \cap \mathbb{S}_{1},\left\langle V^{1}, a\right\rangle \in X \Leftrightarrow a \in(X)_{V^{1}} \Leftrightarrow(X)_{V^{1}} \in V^{1} \Leftrightarrow\left\{V^{1}\right\} \times(X)_{V^{1}} \in$ $W_{V^{1}, \boldsymbol{\beta A}} \otimes V^{1}$. We conclude that $V_{\left\langle V^{1}, a\right\rangle, \boldsymbol{\beta} A \times A}^{1}=W_{V^{1}, \boldsymbol{\beta A}} \otimes V^{1} \in \mathbb{S}_{0}[[a]]$.

For every $x \in \mathbb{S}_{0}[[a]]$ there is $f \in \mathbb{S}_{0}$ such that $x=f\left(\left\langle V^{1}, a\right\rangle\right)$. Let $B:=f[\boldsymbol{\beta} A \times A]$; $B \in \mathbb{S}_{0}$, and by Proposition 3.5, $V_{x, B}^{1}=f\left[V_{\left\langle V^{1}, a\right\rangle, \boldsymbol{\beta} A \times A}^{1}\right] \in \mathbb{S}_{0}[[a]]$. The argument in the proof of Proposition 3.6 is easily modified to show that $\mathbf{s h}_{1}(x)$ is $\in$-definable from $V_{x, B}^{1}$, and hence (because $\mathbb{S}_{0}[[a]] \preccurlyeq \mathbb{V}$ ) it belongs to $\mathbb{S}_{0}[[a]]$.

If $\mathbb{S}_{0} \preccurlyeq \mathbb{U}, a \in \mathbb{U}$ and $\mathbb{U}$ is closed under $\mathbf{s h}_{1}$, then $V_{a, A}^{1}=\mathbf{s h}_{1}\left(W_{a, A}^{1}\right) \in \mathbb{U}$ and so $\mathbb{S}_{0}[[a]] \subseteq \mathbb{U}$.

The sequence $\left\langle V_{a, A}^{1}, a\right\rangle$ is (essentially) an example of what, in later sections, we call the pedigree for $a$ over $A$.

The product of a finite number of ultrafilters is of course defined inductively.

Definition 4.3 Let $U_{1}, \ldots, U_{n}$ be ultrafilters over $I_{1}, \ldots, I_{n}$, respectively. $\bigotimes_{i=1}^{0} U_{i}:=\{\{0\}\}, \bigotimes_{i=1}^{1} U_{i}:=U_{1} ;$ and, for $n \geq 2, \bigotimes_{i=1}^{n} U_{i}$ is an ultrafilter over $I_{1} \times \ldots \times I_{n}$ defined by

$$
X \in \bigotimes_{i=1}^{n} U_{i} \Leftrightarrow\left\{i_{1}:\left\{\left\langle i_{2}, \ldots, i_{n}\right\rangle:\left\langle i_{1}, i_{2}, \ldots, i_{n}\right\rangle \in X\right\} \in \bigotimes_{i=2}^{n} U_{i}\right\} \in U_{1}
$$

$\bigotimes_{i=1}^{n} U_{i}$ is isomorphic to $U_{1} \otimes \bigotimes_{i=2}^{n} U_{i}$, via the map $\left\langle x_{1}, \ldots, x_{n}\right\rangle \mapsto\left\langle x_{1},\left\langle x_{2}, \ldots x_{n}\right\rangle\right\rangle$. If $U_{1}=\ldots=U_{n}=U$, we write $\bigotimes_{i=1}^{n} U_{i}$ as $\otimes^{n} U$.

If $d=\left\{s_{1}, \ldots, s_{k}\right\} \subseteq\{1, \ldots, n\}$, we define the canonical projection $\pi_{d}=\pi_{s_{1}, \ldots, s_{k}}$ : $I_{1} \times \ldots \times I_{n} \rightarrow I_{s_{1}} \times \ldots \times I_{s_{k}}$ by $\pi_{d}\left(\left\langle i_{1}, \ldots, i_{n}\right\rangle\right)=\left\langle i_{s_{1}}, \ldots, i_{s_{k}}\right\rangle ; \pi_{0}\left(\left\langle i_{1}, \ldots, i_{n}\right\rangle\right)=0$. It is easy to verify that $\pi_{d}$ is a morphism from $U_{1} \otimes \ldots \otimes U_{n}$ to $U_{s_{1}} \otimes \ldots \otimes U_{s_{k}}$ (see Section 6 for more general results).

By repeated appeals to Proposition 4.1 one can construe $\mathcal{U} \ell\left(\mathbb{V} ; \bigotimes_{i=1}^{n} U_{i}\right)$ as $n$-times repeated ultrapower of the universe. But, for the construction of interpretations of relative set theory, we need even "transfinite repetitions" of the ultrapower construction. Here we give the simplest example, as a motivation for the general theory of such repetitions that is developed in the subsequent sections.

Let $U$ be a nonprincipal ultrafilter over $\omega$ and let $V:=\Sigma_{U} \bigotimes^{n} U$. Each $\bigotimes^{n} U$ is an ultrafilter over $\omega^{n}$, hence $V$ is the ultrafilter over $K:=\bigcup_{n \in \omega}\{n\} \times \omega^{n}$ defined by 
$X \in V \Leftrightarrow\left\{n \in \omega:(X)_{n} \in \bigotimes^{n} U\right\} \in U$. By the Factoring Lemma, $\mathcal{U} \ell(\mathbb{V} ; V)$ is isomorphic to $[\mathcal{U} \ell(\mathbb{V} ; u)]^{\mathcal{U} \ell(\mathbb{V} ; U)}$ for $u=\left\langle\bigotimes^{n} U: n \in \omega\right\rangle$. We note that $\mathcal{U} \ell(\mathbb{V} ; U) \vDash$ " $u=\bigotimes^{d} \mathfrak{k}_{U}(U)$ " where $d:=I d_{\omega}$ is a "hyperfinite integer": $I d_{\omega} \in_{U} \mathfrak{k}_{U}(\omega), I d_{\omega} \neq_{U}$ $\mathfrak{k}_{U}(n)$ for any $n \in \omega$. The interpretation can thus be viewed as an ultrapower repeated a "hyperfinite" number of times. This idea is presented in full generality in Sections 6 and 7.

\section{Limit Ultrapowers and BST.}

Many realizations of the nonstandard set theory ST are not (isomorphic to) ultrapowers of the universe. They turn out to be (isomorphic to) limit ultrapowers of the universe, if this concept is defined in sufficient generality.

Let $(\mathbb{D}, \leq)$ be a directed preordering [that is, $(\forall d \in \mathbb{D})(d \leq d),\left(\forall d, d^{\prime}, d^{\prime \prime} \in \mathbb{D}\right)(d \leq$ $\left.d^{\prime} \wedge d^{\prime} \leq d^{\prime \prime} \Rightarrow d \leq d^{\prime \prime}\right)$, and $\left.\left(\forall d, d^{\prime} \in \mathbb{D}\right)\left(\exists d^{\prime \prime} \in \mathbb{D}\right)\left(d \leq d^{\prime \prime} \wedge d^{\prime} \leq d^{\prime \prime}\right)\right]$.

Furthermore, let $\mathbb{U}=\left\langle U_{d}: d \in \mathbb{D}\right\rangle$ be a system of ultrafilters, and $\phi=\left\langle\phi_{d, d^{\prime}}: d \leq d^{\prime}\right\rangle$ be such that, for all $d \leq d^{\prime}, \phi_{d, d^{\prime}} \neq 0, \varphi \in \phi_{d, d^{\prime}} \Rightarrow \varphi: U_{d^{\prime}} \rightarrow U_{d}, \varphi, \varphi^{\prime} \in \phi_{d, d^{\prime}} \Rightarrow$ $\varphi={ }_{U_{d^{\prime}}} \varphi^{\prime}$, and $\varphi \in \phi_{d, d^{\prime}}, \varphi^{\prime} \in \phi_{d^{\prime}, d^{\prime \prime}}$ implies $\varphi \circ \varphi^{\prime}=U_{d^{\prime \prime}} \varphi^{\prime \prime}$ for some $\varphi^{\prime \prime} \in \phi_{d, d^{\prime \prime}}$, for $d \leq d^{\prime} \leq d^{\prime \prime}$. We refer to this data as a directed system of ultrafilters.

This data induces a system $\left\langle\mathcal{U} \ell t\left(\mathbb{V} ; U_{d}\right): d \in \mathbb{D}\right\rangle$ of interpretations, and a system $\Phi=\left\langle\Phi_{d, d^{\prime}}: d \leq d^{\prime}\right\rangle$ of morphisms $\Phi_{d, d^{\prime}}: \mathcal{U} \ell t\left(\mathbb{V} ; U_{d}\right) \rightarrow \mathcal{U} \ell t\left(\mathbb{V} ; U_{d^{\prime}}\right)$ [that is, $\in-$ elementary embeddings that commute with the natural embeddings: $\left.\Phi_{d, d^{\prime}} \circ \mathfrak{k}_{U_{d}}=\mathfrak{k}_{U_{d^{\prime}}}\right]$ such that $d \leq d^{\prime} \leq d^{\prime \prime} \Rightarrow \Phi_{d, d^{\prime \prime}}=\Phi_{d^{\prime}, d^{\prime \prime}} \circ \Phi_{d, d^{\prime}}$; namely, $\Phi_{d, d^{\prime}}=\varphi^{*}$ for any $\varphi \in \phi_{d, d^{\prime}}$.

As we do not assume Global Choice, it is not in general possible to choose a unique representative $\varphi_{d, d^{\prime}} \in \phi_{d, d^{\prime}}$. However, in most applications such choice is possible. In any case, we use $\varphi_{d, d^{\prime}}$ as notation for a generic element of $\phi_{d, d^{\prime}}$.

A limit ultrapower of the universe $\mathcal{L U} \ell t(\mathbb{V} ; \mathbb{U}, \phi)$ is the direct limit of this system.

A concrete description of the direct limit as a union of ultrapowers can be given as follows. Without loss of generality we assume that $\operatorname{dom} U_{d} \cap \operatorname{dom} U_{d^{\prime}}=0$ for $d \neq d^{\prime}$. (Replace $I_{d}$ by $\{d\} \times I_{d}$ and $U_{d}$ by its image under the mapping $x \mapsto\langle d, x\rangle$.)

We define $f \in \mathbb{V}^{*}$ iff $(\exists d \in \mathbb{D})\left(f \in \mathbb{V}^{U_{d}}\right)$; that is, $\mathbb{V}^{*}=\bigcup_{d \in \mathbb{D}} \mathbb{V}^{U_{d}}$. For $f \in \mathbb{V}^{U_{d}}, g \in$ $\mathbb{V}^{U_{d^{\prime}}}, f={ }^{*} g$ iff $\left(\exists d^{\prime \prime} \in \mathbb{D}\right)\left(d \leq d^{\prime \prime} \wedge d^{\prime} \leq d^{\prime \prime} \wedge \Phi_{d, d^{\prime \prime}}(f)=_{U_{d}^{\prime \prime}} \Phi_{d^{\prime}, d^{\prime \prime}}(g)\right)$; similarly for $\in^{*} ; f \in \mathbb{S}^{*}$ iff $f={ }^{*} \mathfrak{k}_{U_{d}}(x)$ for some $x \in \mathbb{V} ; \mathcal{L U} \ell t(\mathbb{V} ; \mathbb{U}, \phi):=\left(\mathbb{V}^{*},=^{*}, \in^{*}, \mathbb{S}^{*}\right)$.

We also define $\Phi_{d, *}: \mathbb{V}^{U_{d}} \rightarrow \mathbb{V}^{*}$ by $\Phi_{d, *}(f)=f$ [ie, $\Phi_{d, *}$ is the inclusion of $\mathbb{V}_{U_{d}}$ in $\left.\mathbb{V}^{*}\right]$, and $\mathfrak{k}: \mathbb{V} \rightarrow \mathbb{V}^{*}$ by $\mathfrak{k}(x)=\mathfrak{k}_{U}(x)$ where $U=U_{\bar{d}}$ for a fixed $\bar{d} \in \mathbb{D}$. Note that $d \leq d^{\prime} \Rightarrow \Phi_{d, *}=\Phi_{d^{\prime}, *} \circ \Phi_{d, d^{\prime}}\left[\right.$ this amounts to $f={ }^{*} \Phi_{d, d^{\prime}}(f)$, for $\left.f \in \mathbb{V}^{U_{d}}\right]$. 
Proposition $5.1 \mathcal{L U} \ell t(\mathbb{V} ; \mathbb{U}, \phi)$ is a realization of $\mathbf{S T}$.

For each $d \in \mathbb{D}, \Phi_{d, *}: \mathcal{U} \ell t\left(\mathbb{V} ; \mathbb{U}_{d}\right) \rightarrow \mathcal{L U} \ell t(\mathbb{V} ; \mathbb{U}, \phi)$ is a morphism.

Proof First we show that $\Phi_{d, *}$ is an embedding. For $f, g \in \mathbb{V}^{U_{d}}$ and $d \leq d^{\prime \prime}$, $f=U_{U_{d}} g \Leftrightarrow \Phi_{d, d^{\prime \prime}}(f)=_{U_{d^{\prime \prime}}} \Phi_{d, d^{\prime \prime}}(g)$, so $f==_{U_{d}} g \Leftrightarrow f={ }^{*} g$. Similarly for $\epsilon$. $f \in \mathfrak{k}_{U_{d}}[\mathbb{V}] \Leftrightarrow f \in \mathbb{S}^{*}$ is immediate from the definition. It is straightforward to verify that $=^{*}$ is a congruence. $\Phi_{d, *}$ commutes with the natural embeddings: for $x \in \mathbb{V}$, $\mathfrak{k}(x)=\mathfrak{k}_{U_{\bar{d}}}(x)={ }^{*} \mathfrak{k}_{U_{d}}(x)=\Phi_{d, *}\left(\mathfrak{k}_{U_{d}}(x)\right)$.

A well-known argument by induction on complexity of formulas shows that $\varphi_{d, *}$ is $\in$-elementary (hence, a morphism). The nontrivial case is when $\mathcal{L U} \ell t(\mathbb{V} ; \mathbb{U}, \phi) \vDash$ $(\exists g) \mathcal{P}\left(f_{1}, \ldots, f_{k}, g\right)$, for $f_{1}, \ldots, f_{k} \in \mathbb{V}^{U_{d}}$. We take $d^{\prime} \geq d$ such that $\mathcal{L U} \ell t(\mathbb{V} ; \mathbb{U}, \phi) \vDash$ $\mathcal{P}\left(f_{1}, \ldots, f_{k}, g\right)$ for some $g \in \mathbb{V} U_{d^{\prime}}$. As $f_{i}={ }^{*} \Phi_{d, d^{\prime}}\left(f_{i}\right)$ and $={ }^{*}$ is a congruence, we have also $\mathcal{L U} \ell t(\mathbb{V} ; \mathbb{U}, \phi) \vDash \mathcal{P}\left(\Phi_{d, d^{\prime}}\left(f_{1}\right), \ldots, \Phi_{d, d^{\prime}}\left(f_{k}\right), g\right)$. By the inductive assumption then $\mathcal{U} \ell t\left(\mathbb{V} ; \mathbb{U}_{d^{\prime}}\right) \vDash \mathcal{P}\left(\Phi_{d, d^{\prime}}\left(f_{1}\right), \ldots, \Phi_{d, d^{\prime}}\left(f_{k}\right), g\right)$, ie, $\mathcal{U} \ell t\left(\mathbb{V} ; \mathbb{U}_{d^{\prime}}\right) \vDash$ $(\exists g) \mathcal{P}\left(\Phi_{d, d^{\prime}}\left(f_{1}\right), \ldots, \Phi_{d, d^{\prime}}\left(f_{k}\right), g\right)$. As $\Phi_{d, d^{\prime}}$ is $\in$-elementary, we get $\mathcal{U} \ell t\left(\mathbb{V} ; \mathbb{U}_{d}\right) \vDash$ $(\exists g) \mathcal{P}\left(f_{1}, \ldots, f_{k}, g\right)$.

ZFC holds in $\left(\mathbb{S}^{*},=^{*}, \in^{*}\right)$, which is isomorphic to $(\mathbb{V},=, \in)$ via $\mathfrak{k}$. Boundedness and Inner Standardization hold because they hold in each $\mathcal{U} \ell t\left(\mathbb{V} ; \mathbb{U}_{d}\right)$. Finally, for $f_{1}, \ldots, f_{k} \in \mathbb{S}^{*}$ (without loss of generality $f_{i}=\mathfrak{k}_{U_{d}}\left(x_{i}\right) \in \mathbb{V}^{\mathbb{U}_{d}}$ for some $d$, where $x_{i} \in$ $\mathbb{V}), \mathcal{L U} \ell t(\mathbb{V} ; \mathbb{U}, \phi) \vDash \mathcal{P}^{\text {st }}\left(f_{1}, \ldots, f_{k}\right) \Leftrightarrow(\mathbb{V},=, \in) \vDash \mathcal{P}\left(x_{1}, \ldots, x_{k}\right) \Leftrightarrow \mathcal{U} \ell t\left(\mathbb{V} ; \mathbb{U}_{d}\right) \vDash$ $\mathcal{P}^{\text {st }}\left(f_{1}, \ldots, f_{k}\right) \Leftrightarrow \mathcal{U} \ell t\left(\mathbb{V} ; \mathbb{U}_{d}\right) \vDash \mathcal{P}\left(f_{1}, \ldots, f_{k}\right)$ (Transfer holds in $\left.\mathcal{U} \ell t\left(\mathbb{V} ; \mathbb{U}_{d}\right)\right) \Leftrightarrow$ $\mathcal{L U} \ell t(\mathbb{V} ; \mathbb{U}, \phi) \vDash \mathcal{P}\left(f_{1}, \ldots, f_{k}\right)$ (because $\Phi_{d, *}$ is $\in$-elementary). Hence $\mathcal{L U} \ell t(\mathbb{V} ; \mathbb{U}, \phi)$ satisfies ST.

Proposition 5.2 Every realization of $\mathbf{S T}$ is isomorphic to a limit ultrapower of the universe.

\section{Proof (Outline)}

Let $\Im:=\left(\mathbb{I},=_{\Im}, \in_{\Im}, \mathbb{S}_{\Im}\right)$ be such interpretation and let $\mathfrak{k}$ be the natural isomorphism of $\mathbb{V}$ onto $\mathbb{S}_{\Im}$. Theorem 3.7 holds in $\Im \vDash \mathbf{S T}$. Hence every $x \in \mathbb{I}, x \in_{\Im} \mathfrak{k}(A)$, generates $(\mathbb{S}[x])^{\Im}=\left\{z \in \mathbb{I}: \Im \vDash " z=\mathfrak{k}(f)(x)\right.$ ” for some $\left.f \in \mathbb{V}^{A}\right\}$. There is a unique ultrafilter $U_{x, A}$ over $A$ such that $\Im \vDash$ "k $\left(U_{x, A}\right)$ is generated by $x$ over $\mathfrak{k}(A)$ ", ie, $(\forall X \subseteq A)\left(X \in U_{x, A} \Leftrightarrow x \in_{\Im} \mathfrak{k}(X)\right)$.

Also, $\Im \vDash “ \mathfrak{j}_{x, \mathfrak{k}(A)}$ is an isomorphism of $\left[\mathcal{U} \ell t\left(\mathbb{V} ; \mathfrak{k}\left(U_{x, A}\right)\right)\right]^{\mathcal{S}}$ onto $(\mathbb{S}[x],=, \in, \mathbb{S})$ ”. An examination of this statement in the light of discussions in Section 1 shows that $\left(\mathfrak{j}_{x, \mathfrak{k}(A)}\right)^{\Im}$ is an isomorphism of $\left[\mathcal{U} \ell t\left(\mathbb{V} ; \mathfrak{k}\left(U_{x, A}\right)\right)\right]^{\mathcal{S}_{\Im}}$ onto $\left((\mathbb{S}[x])^{\Im},=\Im, \in_{\Im}, \mathbb{S}_{\Im}\right)$. Also, $\mathfrak{k}$ is the 
natural isomorphism of $\mathbb{V}$ onto $\mathbb{S}^{\Im}$, so it maps $\mathcal{U} \ell t\left(\mathbb{V} ; U_{x, A}\right)$ isomorphically onto $\left[\mathcal{U} \ell t\left(\mathbb{V} ; \mathfrak{k}\left(U_{x, A}\right)\right)\right]^{\mathcal{S}^{\Im}}$.

The idea of the proof is to observe that $\mathbb{I}$ is the union of the system of classes $(\mathbb{S}[x])^{\Im}$ as $x$ ranges over $\mathbb{I}$, directed by inclusion, and to construct an isomorphic directed system of ultrapowers of $\mathbb{V}$.

We let $\mathbb{D}:=\left\{\langle x, A\rangle: x \in \mathbb{I} \wedge x \in_{\Im} \mathfrak{k}(A)\right\}$. For $\langle x, A\rangle,\langle y, B\rangle \in \mathbb{D}$ we define $\langle x, A\rangle \leq\langle y, B\rangle \Leftrightarrow \Im \vDash \mathbb{S}[x] \subseteq \mathbb{S}[y] \Leftrightarrow\left(\forall f \in \mathbb{V}^{A}\right)\left(\exists g \in \mathbb{V}^{B}\right)(\Im \vDash \mathfrak{k}(f)(x)=\mathfrak{k}(g)(y))$. It is immediate that $\leq$ is a directed preordering ( $\vDash \vDash \mathbb{S}[x] \cup \mathbb{S}[y] \subseteq \mathbb{S}[\{x, y\}]$ "). To each $\langle x, A\rangle \in \mathbb{D}$ we assign the ultrafilter $U_{x, A}$. Finally, for $\langle x, A\rangle \leq\langle y, B\rangle$ we let $\Theta:=\mathfrak{k}^{-1} \circ\left(\left(\mathfrak{j}_{y, \mathfrak{k}(B)}\right)^{\Im}\right)^{-1} \circ\left(\mathfrak{j}_{x, \mathfrak{k}(A)}\right)^{\Im} \circ \mathfrak{k}$. It is easily verified that $\Theta$ is a morphism of $\mathcal{U} \ell t\left(\mathbb{V} ; U_{x, A}\right)$ into $\mathcal{U} \ell t\left(\mathbb{V} ; U_{y, B}\right)$, and hence $\Theta=\varphi^{*}$ for some $\varphi: U_{y, B} \rightarrow U_{x, A}$. We let $\phi_{\langle x, A\rangle,\langle y, B\rangle}$ be the set of all such $\varphi$.

It is now a matter of tedious verification (which we skip) to show that the direct limit of the system just described is isomorphic to $\Im$.

The enormous variety of limit ultrapowers leaves little hope for general results beyond Propositions 5.1 and 5.2, which characterize limit ultrapowers as being precisely the realizations of ST. However, we show that there is a realization of ST that is universal in a well-defined sense, and that this universal realization singles out a particular nonstandard set theory known as BST.

We use a particular kind of the limit ultrapower construction, known as the iterated ultrapower.

Let $(\Delta, \leq)$ be a total ordering, that is, $\leq$ is reflexive, antisymmetric and transitive in $\Delta$ and $\left(\forall \delta, \delta^{\prime} \in \Delta\right)\left(\delta \leq \delta^{\prime} \vee \delta^{\prime} \leq \delta\right)$. Let $\left\langle U_{\delta}: \delta \in \Delta\right\rangle$ assign an ultrafilter over $I_{\delta}$ to each $\delta \in \Delta$. By replacing $U_{\delta}$ by $U_{\delta}^{\prime}$ over $I_{\delta}^{\prime}:=\{\delta\} \times I_{\delta}$, where $X \in U_{\delta} \Leftrightarrow\{\delta\} \times X \in U_{\delta}^{\prime}$, we can and do assume that $I_{\delta} \cap I_{\delta^{\prime}}=0$ for $\delta \neq \delta^{\prime}$.

We let $\mathbb{D}:=\mathcal{P}_{\text {fin }}(\Delta)$ be the class of all finite subsets of $\Delta$; the inclusion $\subseteq$ is then a directed ordering of $\mathbb{D}$. To each $d=\left\{\delta_{1}, \ldots, \delta_{n}\right\} \in \mathbb{D}$, where $\delta_{1}<\ldots<\delta_{n}$, we assign the ultrafilter $U_{d}:=U_{\delta_{1}} \otimes \ldots \otimes U_{\delta_{n}}$ over $K_{d}:=I_{\delta_{1}} \times \ldots \times I_{\delta_{n}} ; U_{0}:=\{\{0\}\}$. Let $\mathbb{U}:=\left\langle U_{d}: d \in \mathbb{D}\right\rangle$. For $d \subseteq d^{\prime}$ we let $\varphi_{d, d^{\prime}}$ be the canonical projection of $U_{d^{\prime}}$ onto $U_{d}$ (see Section 4); let $\phi=\left\langle\left\{\varphi_{d, d^{\prime}}\right\}: d \subseteq d^{\prime}\right\rangle . \mathcal{L U} \ell t(\mathbb{V} ; \mathbb{U}, \phi)$ is called the iterated ultrapower of the universe along $\left\langle U_{\delta}: \delta \in \Delta\right\rangle$.

Iterated ultrapowers were introduced by Gaifman [9], see also Chang-Keisler [6], and extensively used by Kunen [26] and others in the study of large cardinals. In 
such applications the ultrafilters $U_{\delta}$ are usually $\omega_{1}$-complete. Our main interest here is in constructing an iterated ultrapower along a total ordering $\Delta$ that indexes all ultrafilters. This is easy to do if one assumes Global Choice; such iterations were used by the author to construct interpretations of nonstandard set theories in [11]. Kanovei $[23,22]$ employed an observation of Shelah to define such iterations in ZFC; we follow his method below.

Let $\kappa$ be an infinite cardinal. The set $\mathcal{P}(\kappa)$ is totally ordered lexicographically: for $X, Y \in \mathcal{P}(\kappa), X \leq Y \Leftrightarrow X=Y \vee \sigma \in Y$, where $\sigma$ is the least element of the symmetric difference of $X$ and $Y$. Furthermore, the set $\Delta_{\kappa}:=\mathcal{P}(\kappa)^{2^{\kappa}}$ is totally ordered lexicographically: for $\alpha, \beta: 2^{\kappa} \rightarrow \mathcal{P}(\kappa)$ we let $\alpha \leq_{\kappa} \beta \Leftrightarrow \alpha=\beta \vee \alpha(\rho)<\beta(\rho)$, where $\rho$ is the least element of $\left\{\tau<2^{\kappa}: \alpha(\tau) \neq \beta(\tau)\right\}$.

We let $\bar{\Delta}:=\bigcup_{\kappa}\{\kappa\} \times \Delta_{\kappa}$ and define a total ordering $\leq$ on $\bar{\Delta}$ by: $\langle\kappa, \alpha\rangle \leq\left\langle\kappa^{\prime}, \beta\right\rangle$ iff $\kappa>\kappa^{\prime} \vee\left(\kappa=\kappa^{\prime} \wedge \alpha \leq_{\kappa} \beta\right)$. Note the reversal of the order of cardinals!

To each $\delta=\langle\kappa, \alpha\rangle \in \bar{\Delta}$ we assign an ultrafilter $U_{\delta}$ as follows: $U_{\delta}=\operatorname{ran} \alpha$ if $\operatorname{ran} \alpha$ is an ultrafilter over $\kappa ; U_{\delta}=\{\{0\}\}$ otherwise. Note that every ultrafilter over $\kappa$ occurs (repeatedly) as $U_{\delta}$ for some $\delta=\langle\kappa, \alpha\rangle \in \bar{\Delta}$.

Let $\mathfrak{M}:=\left(\mathbb{V}^{*},=^{*}, \in^{*}, \mathbb{S}^{*}\right)$ be the iterated ultrapower of the universe along $\left\langle U_{\delta}: \delta \in\right.$ $\bar{\Delta}\rangle$, and $\mathfrak{k}$ the canonical embedding of $\mathbb{V}$ into $\mathbb{V}^{*}$. According to Proposition 5.1, $\mathfrak{M}$ is a realization of $\mathbf{S T}$.

The Back and Forth Property is the conjunction of the following two statements in the $\{\in$, st $\}$-language:

(B)

$(\forall x, y)\left(\forall^{\text {st }} U, F\right)\left[(\operatorname{ran} F \subseteq \operatorname{dom} U \wedge x \mathbb{M} U \wedge x=F(y)) \Rightarrow\left(\exists^{\text {st }} V\right)(U=F[V] \wedge y \mathbb{M} V)\right]$

$(\mathbf{F})$

$(\forall x)\left(\forall^{\text {st }} U, V, F\right)[(\operatorname{ran} F \subseteq \operatorname{dom} U \wedge x \mathbb{M} U \wedge U=F[V]) \Rightarrow(\exists y)(y \mathbb{M} V \wedge x=F(y))]$.

In particular, B implies that for every $y$ there is a standard $V$ such that $y \mathbb{M} V$ (take $U=\{\{0\}\}, x=0$ ). This $V$ is nothing but $V_{y, \operatorname{dom} V}$, and so $\mathbf{B}$ implies (over $\mathbf{S T}^{-}$) that Inner Standardization holds, by Proposition 3.6.

By a similar argument, $\mathbf{F}$ implies that for every standard $V$ there is $y$ that generates $V$ (over $\operatorname{dom} V$ ).

Proposition 5.3 $\mathfrak{M}$ satisfies the Back and Forth Property. 
Proof $\mathfrak{M} \vDash \mathbf{S T}$, and $\mathbf{B}$ follows from $\mathbf{S T}$, as shown in Propositions 3.6 and 3.5.

Let $U, V$ be ultrafilters, $U=F[V]$ and $\mathfrak{M} \vDash f \mathbb{M k}(U)$. Fix finite $d \subseteq \bar{\Delta}$ such that $f \in \mathbb{V}^{U_{d}}$ (recall $U_{d}=U_{\delta_{1}} \otimes \ldots \otimes U_{\delta_{n}}$ where $d=\left\{\delta_{1}, \ldots, \delta_{n}\right\}$ in $\leq$-increasing order, and $\delta_{i}=\left\langle\kappa_{i}, \alpha_{i}\right\rangle$ ). Let $\bar{U}$ be a $\lambda$-good ultrafilter over $\lambda$, for $\lambda>\kappa_{1},|U|,|V|$. We fix $\alpha_{0}$ such that $\bar{U}=U_{\left\langle\lambda, \alpha_{0}\right\rangle}$. Let $\bar{\delta}:=\left\langle\lambda, \alpha_{0}\right\rangle$ and $\bar{d}:=\{\bar{\delta}\} \cup \delta$. Then $U_{\bar{d}}$ is isomorphic to $U_{\bar{\delta}} \otimes U_{d}$, hence it is $\lambda$-good, and $\mathcal{U} \ell\left(\mathbb{V} ; U_{\bar{d}}\right)$ satisfies $\lambda$-Idealization. Of course, also $\mathcal{U} \ell\left(\mathbb{V} ; U_{\bar{d}}\right) \vDash$ "ST $\wedge f \mathbb{M} \mathfrak{k}(U) \wedge \mathfrak{k}(U)=\mathfrak{k}(F)[\mathfrak{k}(V)]$ ". It follows that there is $g \in \mathbb{V}_{\bar{d}}$ such that $\mathcal{U} \ell\left(\mathbb{V} ; U_{\bar{d}}\right) \vDash " g \mathbb{M} \mathfrak{k}(V) \wedge f=\mathfrak{k}(F)(g)$ ", ie, $\mathcal{U} \ell\left(\mathbb{V} ; U_{\bar{d}}\right) \vDash g \in \mathfrak{k}_{U_{\bar{d}}}(Y)$ for every $Y \in V$. Hence $\mathfrak{M} \vDash g \in \mathfrak{k}(Y)$ for every $Y \in V$, ie, $\mathfrak{M} \vDash g \mathbb{M} \mathfrak{k}(V)$ (and $\mathfrak{M} \vDash \mathfrak{k}(U)=\mathfrak{k}(F)[\mathfrak{k}(V)])$. This proves that $\mathbf{F}$ holds in $\mathfrak{M}$.

Proposition 5.4 (ST + Back and Forth Property) (Normal Form Theorem)

There is an effective procedure that assigns to each $\{\in$-st $\}$-formula $\mathcal{P}\left(x_{1}, \ldots, x_{k}\right)$ an $\in$-formula $\mathcal{P}^{s}(U)$ such that, for all $x_{1}, \ldots, x_{k}$ and standard $U$ with $\left\langle x_{1}, \ldots, x_{k}\right\rangle \mathbb{M} U$, $\mathcal{P}\left(x_{1}, \ldots, x_{k}\right) \Leftrightarrow \mathcal{P}^{s}(U)$. In particular,

$$
\begin{gathered}
\mathcal{P}\left(x_{1}, \ldots, x_{k}\right) \Leftrightarrow\left(\exists^{\mathrm{st}} U\right)\left(\left\langle x_{1}, \ldots, x_{k}\right\rangle \mathbb{M} U \wedge \mathcal{P}^{s}(U)\right) \Leftrightarrow \\
\left(\forall^{\text {st }} U\right)\left(\left\langle x_{1}, \ldots, x_{k}\right\rangle \mathbb{M} U \Rightarrow \mathcal{P}^{s}(U)\right) .
\end{gathered}
$$

It follows that each $\mathcal{P}(\bar{x})$ is equivalent to a formula in $\Sigma_{2}^{\text {st }}$ form: $\mathcal{P}\left(x_{1}, \ldots, x_{k}\right) \Leftrightarrow$ $\left(\exists^{\text {st }} U\right)\left(\forall^{\text {st }} X\right)\left[U\right.$ is an ultrafilter $\left.\wedge\left(X \in U \Rightarrow\left\langle x_{1}, \ldots, x_{k}\right\rangle \in X \wedge \mathcal{P}^{s}(U)\right)\right]$, and to a formula in $\Pi_{2}^{\text {st }}$ form: $\mathcal{P}\left(x_{1}, \ldots, x_{k}\right) \Leftrightarrow\left(\forall^{\text {st }} U\right)\left(\exists^{\text {st }} X\right)[U$ is not an ultrafilter $\vee(X \in$ $\left.\left.U \wedge\left\langle x_{1}, \ldots, x_{k}\right\rangle \notin X\right) \vee \mathcal{P}^{s}(U)\right]$.

The first result of this nature was proved by Nelson [27] for IST (Reduction Algorithm). Kanovei adapted it to BST in [20] (see also [22]). The formulation of the Normal Form Theorem given here is due to Andreev [1] (see also [2]), who proved it in BST with only a weak version of Standardization. The proof below is in [3] (see also [15]).

Proof Let $\mathcal{P}\left(x_{1}, \ldots, x_{k}\right)$ be an $\in$-st-formula where all free variables are among $x_{1}, \ldots, x_{k}$. Renaming the bound variables if necessary, we can assume that all bound variables are distinct from all free variables and from each other (ie, if $Q_{1} y_{1}$ and $Q_{2} y_{2}$ are distinct occurrences of quantifiers in $\mathcal{P}$, then $y_{1}$ and $y_{2}$ are distinct variables).

We proceed by induction on the complexity of $\mathcal{P}$. Let $1 \leq i, j \leq k$.

( $\left.x_{i} \in x_{j}\right)^{s}$ is the formula expressing " $\left\{\left\langle a_{1}, \ldots, a_{k}\right\rangle \in \operatorname{dom} U: a_{i} \in a_{j}\right\} \in U$ ";

$\left(x_{i}=x_{j}\right)^{s}$ is the formula " $\left\{\left\langle a_{1}, \ldots, a_{k}\right\rangle \in \operatorname{dom} U: a_{i}=a_{j}\right\} \in U$ ";

$\left(\text { st } x_{i}\right)^{s}$ is " $(\exists a)\left\{\left\langle a_{1}, \ldots, a_{k}\right\rangle \in \operatorname{dom} U: a_{i}=a\right\} \in U$ "; 
$(\mathcal{P} \wedge \mathcal{Q})^{s}$ is $\mathcal{P}^{s} \wedge \mathcal{Q}^{s} ; \quad(\neg \mathcal{P})^{s}$ is $\neg \mathcal{P}^{s} ;$

$\left((\exists y) \mathcal{Q}\left(x_{1}, \ldots, x_{k}, y\right)\right)^{s}$ is $(\exists V)\left(\pi_{1, \ldots, k}[V]=U \wedge \mathcal{Q}^{s}(V)\right)$.

(Without loss of generality, we can assume that $y$ is the last variable on the list.) The Back and Forth Property makes the translation of existential quantifiers work correctly.

Corollary 5.5 (ST + Back and Forth Property) (Boldface Normal Form Theorem) Let $\mathcal{P}\left(a, x_{1}, \ldots, x_{k}\right)$ be any $\{\in$-st $\}$-formula; there is an $\in$-formula $\mathcal{Q}(a, U)$ such that, for all $x_{1}, \ldots, x_{k}$ and standard $U$ with $\left\langle x_{1}, \ldots, x_{k}\right\rangle \mathbb{M} U$,

$$
\left(\forall^{\text {st }} a\right)\left(\mathcal{P}\left(a, x_{1}, \ldots, x_{k}\right) \Leftrightarrow \mathcal{Q}(a, U)\right) .
$$

Proof Let $\mathcal{Q}(a, U)$ be the formula $\mathcal{P}^{s}\left(W_{a, A} \otimes U\right)$, where $A:=\{a\}$.

Kanovei [20] formulated the nonstandard set theory BST (Bounded Set Theory), a modification of Nelson's IST.

\section{Axioms of BST:}

$\mathcal{P}^{\text {st }}$, where $\mathcal{P}$ is any axiom of $\mathbf{Z F C}$.

Boundedness: $\quad(\forall x)\left(\exists^{\text {st }} y\right)(x \in y)$.

Transfer: $\quad\left(\forall^{\text {st }} x_{1}, \ldots, x_{k}\right)\left(\mathcal{P}^{\text {st }}\left(x_{1}, \ldots, x_{k}\right) \Leftrightarrow \mathcal{P}\left(x_{1}, \ldots, x_{k}\right)\right)$

where $\mathcal{P}\left(x_{1}, \ldots, x_{k}\right)$ is any $\in$-formula.

Standardization: $\quad(\forall \bar{x})\left(\forall^{\mathbf{s t}} x\right)\left(\exists^{\text {st }} y\right)\left(\forall^{\mathbf{s t}} z\right)(z \in y \Leftrightarrow z \in x \wedge \mathcal{P}(z, x, \bar{x}))$

where $\mathcal{P}(z, x, \bar{x})$ is any $\in$-st-formula.

\section{Bounded Idealization:}

$(\forall \bar{x})\left(\forall^{\text {st }} A\right)\left[\left(\forall^{\mathbf{s t}} a \in \mathcal{P}^{\text {fin }} A\right)(\exists y)(\forall x \in a) \mathcal{P}(x, y, A, \bar{x}) \Leftrightarrow(\exists y)\left(\forall^{\text {st }} x \in A\right) \mathcal{P}(x, y, A, \bar{x})\right]$ where $\mathcal{P}(x, y, A, \bar{x})$ is any $\in$-formula.

Proposition 5.6 BST is equivalent to $\mathrm{ST}^{-}+$Back and Forth Property.

\section{Proof $\Longrightarrow$}

BST includes the axioms of $\mathbf{S T}^{-}$.

BST $\vdash$ Inner Standardization:

For any $x$, there is a standard set $A$ such that $x \subseteq A$ (Boundedness, (Union) ${ }^{\text {st }}$ ). By Standardization, there is a standard set $a$ such that, for all standard $z, z \in a \Leftrightarrow z \in$ $A \wedge z \in x \Leftrightarrow z \in x$. 
B now follows from Propositions 3.6 and 3.5. (If $x \mathbb{M} U$ and $x=F(y)$, let $A:=\operatorname{dom} U$, $B:=F^{-1}[A]$, and $V$ the ultrafilter generated by $y$ over $B$; then $U=F[V]$.)

$\mathbf{B S T} \vdash\left(\forall^{\mathbf{s t}} \kappa\right)(\kappa$-Idealization $)$ :

Let $\mathcal{P}(x, y, A, R)$ be the formula " $\langle x, y\rangle \in R$ ” and apply Bounded Idealization.

Proposition 3.12 shows that $\left(\forall^{\mathbf{s t}} \kappa\right)(\kappa$-Idealization $)$ implies $\mathbf{F}$.

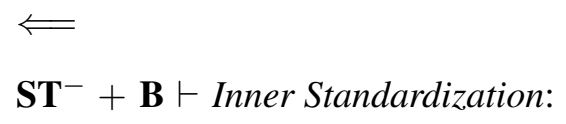

As pointed out in the remark after the statement of the Back and Forth Property, B implies that Inner Standardization holds.

ST $^{-}+$Back and Forth Property $\vdash$ Standardization ([3], Proposition 3):

Let $\mathcal{P}\left(z, x, x_{1}, \ldots, x_{k}\right)$ be any formula. By the Normal Form Theorem, there is an $\in$-formula $\mathcal{P}^{s}(U)$ such that $\mathcal{P}(z, x, \bar{x}) \Leftrightarrow\left(\exists^{\text {st }} U\right)\left(\langle z, x, \bar{x}\rangle \mathbb{M} U \wedge \mathcal{P}^{s}(U)\right)$. We fix a standard ultrafilter $U_{0}$ such that $\langle x, \bar{x}\rangle \mathbb{M} U_{0}$. It is easy to verify that, for any standard $z$ and $U,\langle z, x, \bar{x}\rangle \mathbb{M} U \Leftrightarrow\left(U_{0} \cap \pi_{2, \ldots, k+2}[U]\right)$ is an ultrafilter $\wedge\{\langle w, v, \bar{v}\rangle \in \operatorname{dom} U$ : $w=z\} \in U$. Using Transfer we have that, for standard $z, \mathcal{P}(z, x, \bar{x}) \Leftrightarrow(\exists U)\left[\left(U_{0} \cap\right.\right.$ $\left.\pi_{2, \ldots, k+2}[U]\right)$ is an ultrafilter $\left.\wedge\{\langle w, v, \bar{v}\rangle \in \operatorname{dom} U: w=z\} \in U \wedge \mathcal{P}^{s}(U)\right]$, and the formula on the right side is an $\in$-formula.

$\mathbf{S T}+\mathbf{F} \vDash\left(\forall^{\mathbf{s t}} \kappa\right)(\kappa$-Idealization $)$ :

Let $R \subseteq A \times B, A, B$ standard, and $\left(\forall^{\text {st }} a \in \mathcal{P}^{\text {fin }} A\right)(\exists y)(\forall x \in a)(\langle x, y\rangle \in R)$. Let $U$ be the ultrafilter generated by $R$ over $\mathcal{P}(A \times B)$. For $x \in A$ define $S_{x}:=\{\langle y, r\rangle \in$ $B \times \mathcal{P}(A \times B):\langle x, y\rangle \in r\}$. The collection $\left\{S_{x}: x \in A\right\} \cup\{B \times Z: Z \in U\}$ is standard. The assumptions imply that it has the finite intersection property, so it extends to a standard ultrafilter $V$ over $B \times \mathcal{P}(A \times B)$; obviously, $U=\pi_{2}[V]$. From $R \mathbb{M} U$, using $\mathbf{F}$, we deduce the existence of $\langle y, r\rangle \mathbb{M} V$ such that $\pi_{2}(\langle y, r\rangle)=R$. Then $r=R$ and $\langle y, R\rangle \in S_{x}$, ie, $\langle x, y\rangle \in R$, for all standard $x \in A$.

\section{ST + Back and Forth Property $\vdash$ Bounded Idealization:}

Assume that the left side holds. Selection implies the existence of a set $B$ such

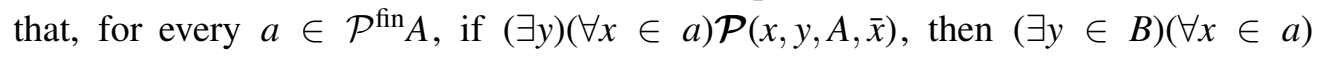
$\mathcal{P}(x, y, A, \bar{x})$; by Boundedness, we can take $B$ to be standard. Define $R:=\{\langle x, y\rangle \in$ $A \times B: \mathcal{P}(x, y, A, \bar{x})\}$ and apply $\kappa$-Idealization to obtain the right side. The converse implication is easy: by Proposition 3.11, if $a \in \mathcal{P}^{\text {fin }} A \cap \mathbb{S}$, then $a \subseteq A \cap \mathbb{S}$.

Corollary 5.7 (Kanovei) BST has a realization in ZFC. 
These results have interesting consequences for model theory of BST. In the rest of this section we use model-theoretic terminology (Chang-Keisler [6]); in particular, a model (of ZFC or BST) is a set, has a satisfaction relation, and satisfies all (formal) axioms (of ZFC or BST).

Corollary 5.8 Every model $\mathbf{M}$ of ZFC can be extended to a model $\mathbf{N}$ of $\mathbf{B S T}$ with $\mathbb{S}^{\mathbf{N}}=\mathbf{M}$.

Corollary 5.9 If $\mathbf{N}_{1}, \mathbf{N}_{2}$ are models of BST and $\mathbf{N}_{1}\left\lceil\mathbb{S}^{\mathbf{N}_{1}}=\mathbf{N}_{2}\left\lceil\mathbb{S}^{\mathbf{N}_{2}}=\mathbf{M}\right.\right.$, then $\mathbf{N}_{1}$ and $\mathbf{N}_{2}$ are $\mathcal{L}_{\infty, \omega}$-elementarily equivalent (where $\mathcal{L}$ is the language with $\in$, st and a name for each $x \in \mathbf{M}$ ). In particular, if also $\left|\mathbf{N}_{1}\right|=\left|\mathbf{N}_{2}\right|=\aleph_{0}$, then $\mathbf{N}_{1}$ and $\mathbf{N}_{2}$ are isomorphic by an isomorphism which is the identity on $\mathbf{M}$.

Proof Define a relation $R$ on $\mathbf{N}_{1}^{<\infty} \times \mathbf{N}_{2}^{<\infty}$ by:

$\left\langle x_{1}^{1}, \ldots, x_{k}^{1}\right\rangle R\left\langle x_{1}^{2}, \ldots, x_{k}^{2}\right\rangle$ iff

$(\exists U \in \mathbf{M})\left[\mathbf{N}_{1} \vDash\left\langle x_{1}^{1}, \ldots, x_{k}^{1}\right\rangle \mathbb{M} U \wedge \mathbf{N}_{2} \vDash\left\langle x_{1}^{2}, \ldots, x_{k}^{2}\right\rangle \mathbb{M} U\right]$.

(Here $\left\langle x_{1}^{1}, \ldots, x_{k}^{1}\right\rangle$ in the scope of $\vDash$ is understood to be evaluated in the model.)

By Boldface Normal Form Theorem, $\left\langle\bar{x}^{1}\right\rangle R\left\langle\bar{x}^{2}\right\rangle$ implies that $\bar{x}^{1}$ and $\bar{x}^{2}$ satisfy the same formulas of the language $\mathcal{L}$. Back and Forth Property shows that $\left\langle\bar{x}^{1}\right\rangle R\left\langle\bar{x}^{2}\right\rangle$ implies $\left(\forall y^{1} \in \mathbf{N}_{1}\right)\left(\exists y^{2} \in \mathbf{N}_{2}\right)\left(\left\langle\bar{x}^{1}, y^{1}\right\rangle R\left\langle\bar{x}^{2}, y^{2}\right\rangle\right)$ and $\left(\forall y^{2} \in \mathbf{N}_{2}\right)\left(\exists y^{1} \in \mathbf{N}_{1}\right)\left(\left\langle\bar{x}^{1}, y^{1}\right\rangle R\left\langle\bar{x}^{2}, y^{2}\right\rangle\right)$. It is well-known [8] that the existence of such a back-and-forth relation between two structures implies their $\mathcal{L}_{\infty, \omega}$-elementary equivalence.

Corollary 5.10 Every countable model of ZFC has a unique (up to isomorphism) extension to a countable model of BST.

Corollary 5.11 If $\mathbf{N}_{1} \vDash \mathbf{S T}, \mathbf{N}_{2} \vDash \mathbf{B S T}, \mathbf{N}_{1} \uparrow \mathbb{S}^{\mathbf{N}_{1}}=\mathbf{N}_{2} \uparrow \mathbb{S}^{\mathbf{N}_{2}}=\mathbf{M}$, and also $\left|\mathbf{N}_{1}\right|=\left|\mathbf{N}_{2}\right|=\aleph_{0}$, then there is an $\in$-elementary embedding of $\mathbf{N}_{1}$ into $\mathbf{N}_{2}$ which is the identity on $\mathbf{M}$.

Proof This is a "one-sided" version of Corollary 5.9.

Corollary 5.12 (Completeness of BST over ZFC) If $\mathbf{T} \supseteq \mathbf{Z F C}$ is a complete consistent theory (in the $\in$-language), then $\mathbf{T}+\mathbf{B S T}$ is a complete consistent theory (in the $\in$-st-language). 
Proof Suppose that $\mathbf{T}_{1}:=\mathbf{T}+\mathbf{B S T}+\mathcal{P}$ and $\mathbf{T}_{2}:=\mathbf{T}+\mathbf{B S T}+\neg \mathcal{P}$ are consistent theories. Let $\mathbf{N}_{1}, \mathbf{N}_{2}$ be models of $\mathbf{T}_{1}, \mathbf{T}_{2}$ resp., and let $\mathbf{M}_{i}:=\mathbf{N}_{i} \uparrow \mathbb{S}^{\mathbf{N}_{i}}$ (viewed as structures for the $\in$-language); then $\mathbf{M}_{i} \vDash \mathbf{T}$. By the Isomorphism Theorem of Shelah [6, Theorem 6.1.15] there is an ultrafilter $U$ such that $\mathcal{U} \ell\left(\mathbf{M}_{1} ; U\right)$ and $\mathcal{U} \ell\left(\mathbf{M}_{2} ; U\right)$ are isomorphic. We let $\overline{\mathbf{N}}_{1}:=\mathcal{U} \ell\left(\mathbf{N}_{1} ; U\right)$ and $\overline{\mathbf{N}}_{2}:=\mathcal{U} \ell\left(\mathbf{N}_{2} ; U\right)$. Then $\overline{\mathbf{N}}_{1} \vDash \mathbf{T}_{1}, \overline{\mathbf{N}}_{2} \vDash \mathbf{T}_{2}$, so $\overline{\mathbf{N}}_{1}$ and $\overline{\mathbf{N}}_{2}$ are not elementarily equivalent, but $\mathbf{N}_{1} \uparrow \mathbb{S}^{\mathbf{N}_{1}}=\mathbf{M}_{1}$ is isomorphic to $\mathbf{N}_{2}\left\lceil\mathbb{S}^{\overline{\mathbf{N}}_{2}}=\mathbf{M}_{2}\right.$. This contradicts Corollary 5.9.

Our goal in the rest of the paper is to obtain results analogous to those of Sections 2-5 for theories with many levels of standardness.

\section{Trees of Ultrafilters.}

The ultrapower construction can be repeated any finite number of times, by straightforward induction. However, the considerations in the subsequent chapters require "hyperfinite" repetitions: if $U$ is nonprincipal, $\mathcal{U} \ell(\mathbb{V} ; U)$ has "hyperfinite" or "nonstandard" natural numbers; ie, there exist $\nu$ such that $\mathcal{U} \ell(\mathbb{V} ; U) \vDash " \nu \in \mathfrak{k}(\omega) \wedge \nu \neq \mathfrak{k}(n)$ ", for all $n \in \omega$; and we have to construct " $\nu$-times repeated" ultrapowers inside $\mathcal{U} \ell(\mathbb{V} ; U)$. This chapter sets up notation and terminology to support this kind of construction.

Finite sequences are elements of $\mathbb{V}<\omega:=\bigcup_{n \in \omega} \mathbb{V}^{n}$. If $t \in \mathbb{V}^{n},|t|=n=\operatorname{dom} t$ is the length of $t .\langle i\rangle:=\{(0, i)\}$ is the finite sequence of length 1 with value $i$. For $t, s \in \mathbb{V}<\omega$, the concatenation $t \frown s \in \mathbb{V}<\omega$ is defined by

$$
(t \frown s)(k)= \begin{cases}t(k) & \text { for } k<|t| ; \\ s(\ell) & \text { for } k=|t|+\ell, \ell<|s| .\end{cases}
$$

A tree is a nonempty set $T$ of finite sequences closed under subsequences (ie, $t \in T$, $k<|t| \Rightarrow t \uparrow k \in T)$ and well-founded under $\supseteq[\mathrm{ie}, \neg(\exists f)(\operatorname{dom} f=\omega \wedge(\forall n \in$ $\omega)(f\lceil n \in T))]$.

The requirement of well-foundedness assigns to each tree an ordinal number, its rank. To make this explicit, we restate the definition of trees in recursive form.

(0) $T=\{0\}$ is a tree of rank 0 .

(1) If $I \neq 0$ and $T_{i}$ is a tree for each $i \in I$, then $T:=\{0\} \cup \bigcup_{i \in I}\langle i\rangle \frown T_{i}$ is a tree, and $\operatorname{rank}(T)=\sup \left\{\operatorname{rank}\left(T_{i}\right)+1: i \in I\right\}$.

It is routine to show that the smallest class closed under (0) and (1) is precisely the class of all trees. 
$t \in T$ is a leaf iff it is $\subseteq$-maximal, ie, there is no $t^{\prime} \in T$ with $t \subset t^{\prime}$.

$\Sigma T$ is the set of all leaves of $T$.

For $t \in T$ let

$T_{t}:=\{s: t \frown s \in T\} \quad$ (the $t$-branch of $T$ );

$[T]_{t}:=\{i: t \frown\langle i\rangle \in T\} \quad$ (the $t$-level of $T$ ).

It is clear that $T_{t}$ is a tree and $T=\{0\} \cup \bigcup_{i \in[T]_{0}}\langle i\rangle \frown T_{\langle i\rangle}$. Also, $t \in \Sigma T \Leftrightarrow T_{t}=$ $\{0\} \Leftrightarrow[T]_{t}=0$.

If $F$ is a function with $\operatorname{dom} F \subseteq T$, we define $F_{t}$ with $\operatorname{dom} F_{t} \subseteq T_{t}$ and $[F]_{t}$ with $\operatorname{dom}[F]_{t} \subseteq[T]_{t}$ by $F_{t}(s)=F(t \frown s)$ and $[F]_{t}(i)=F(t \frown\langle i\rangle)$, resp. $F_{t}$ and $[F]_{t}$ are "restrictions" of $F$ to the branch $T_{t}$ and the level $[T]_{t}$, resp.

Similarly, for $X \subseteq T,(X)_{t}:=\{s: t \frown s \in X\}$ and $[X]_{t}:=\{i: t \frown\langle i\rangle \in X\}$.

If $t=\langle i\rangle$ and there is no danger of misunderstanding, we write $T_{i},[T]_{i}, F_{i},[F]_{i}$ etc. for $T_{\langle i\rangle},[T]_{\langle i\rangle}, F_{\langle i\rangle},[F]_{\langle i\rangle}$, resp.

$A \downarrow:=\{t\lceil n: t \in A, n \in \omega\}$ is a tree, if $0 \neq A \subseteq T$.

Definition 6.1 Let $T$ be a tree. A tree of ultrafilters (TOU) over $T$ is a function $\mathcal{U}$ with $\operatorname{dom} \mathcal{U}=T \backslash \Sigma T$ such that, for each $t \in \operatorname{dom} \mathcal{U}, \mathcal{U}(t)$ is a nonprincipal ultrafilter over $[T]_{t}$.

An equivalent recursive definition is:

(0) $\mathcal{U}=0$ is a TOU over $\{0\}$;

(1) If $T=\{0\} \cup \bigcup_{i \in I}\langle i\rangle \frown T_{i}$, $\operatorname{dom} \mathcal{U} \subseteq T, \mathcal{U}(0)$ is a nonprincipal ultrafilter over $I$ and, for each $i \in I, \mathcal{U}_{\langle i\rangle}$ is a TOU over $T_{i}$, then $\mathcal{U}$ is a TOU over $T$.

We note that the tree $T$ is determined by $\mathcal{U}(t \in T \Leftrightarrow t \in \operatorname{dom} \mathcal{U} \vee t=s \frown\langle i\rangle$ for $s \in \operatorname{dom} \mathcal{U}, i \in \operatorname{dom} \mathcal{U}(s))$; we denote it $T_{\mathcal{U}}$ if necessary for clarity. Also, $\mathcal{U}_{t}$ is a TOU, for each $t \in \operatorname{dom} \mathcal{U}$.

The ultrafilter $\Sigma \mathcal{U}$ over $\Sigma T$ is defined recursively.

Definition 6.2 (0) If $\mathcal{U}=0, \Sigma \mathcal{U}$ is the principal ultrafilter $\{\{0\}\}$ over $\Sigma T=\{0\}$.

(1) If $T=\{0\} \cup \bigcup_{i \in I}\langle i\rangle \frown T_{i}$, then $\Sigma T=\bigcup_{i \in I}\langle i\rangle \frown \Sigma T_{i}$, and for $X \subseteq \Sigma T$ we let

$$
\begin{aligned}
X \in \Sigma \mathcal{U} \Leftrightarrow\left\{i \in I:(X)_{\langle i\rangle} \in \Sigma \mathcal{U}_{\langle i\rangle}\right\} & \in \mathcal{U}(0) \\
& \Leftrightarrow\left\{i \in I:\left\{s \in \Sigma T_{i}:\langle i\rangle \frown s \in X\right\} \in \Sigma \mathcal{U}_{\langle i\rangle}\right\} \in \mathcal{U}(0) .
\end{aligned}
$$


Thus $\Sigma \mathcal{U}$ is isomorphic to the Rudin-Frolík sum of the ultrafilters $\Sigma \mathcal{U}_{\langle i\rangle}$ modulo $\mathcal{U}(0)$, $\Sigma_{\mathcal{U}(0)}\left(\Sigma \mathcal{U}_{\langle i\rangle}\right)$, via the one-one map $\langle i\rangle \frown s \mapsto\langle i, s\rangle$. In particular, if $\operatorname{rank} T=1, \Sigma \mathcal{U}$ is isomorphic to $\mathcal{U}(0)$ via the one-one map $\langle i\rangle \mapsto i$ of $\Sigma T$ onto $[T]_{0}$.

We reserve letters $\varphi, \psi, \theta$ for mappings from a tree to a tree. For mappings denoted by these letters only, we modify our notational conventions as follows.

Let $\varphi: \subseteq T^{2} \rightarrow T^{1}, t \in \operatorname{dom} \varphi$. $\varphi_{t}: \subseteq T_{t}^{2} \rightarrow T_{\varphi(t)}^{1}$ is defined by $\varphi(t \frown s)=\varphi(t) \frown \varphi_{t}(s)$;

$[\varphi]_{t}: \subseteq\left[T^{2}\right]_{t} \rightarrow\left[T^{1}\right]_{\varphi(t)}$ is defined by $\varphi(t \frown\langle i\rangle)=\varphi(t) \frown\left\langle[\varphi]_{t}(i)\right\rangle$.

We note that $\varphi_{t}(s)$ is defined iff $\varphi(t) \subseteq \varphi(t \frown s)$, and $[\varphi]_{t}(i)$ is defined iff $\varphi(t \frown$ $\langle i\rangle)=\varphi(t) \frown\langle j\rangle$ for some $j$; when this is the case, $[\varphi]_{t}(i)=j$.

Definition 6.3 $\varphi$ is a morphism of $\mathcal{U}^{2}$ to $\mathcal{U}^{1}$ (notation $\varphi: \mathcal{U}^{2} \rightarrow \mathcal{U}^{1}$ ) iff $\operatorname{dom} \varphi \subseteq T^{2}$ is a tree, and

(i) $\varphi(0)=0$;

(ii) for each $t \in \operatorname{dom} \varphi \backslash \Sigma T^{2}$, $[\operatorname{dom} \varphi]_{t} \in \mathcal{U}^{2}(t)$, and either

(c) $\varphi(t \frown\langle j\rangle)=\varphi(t)$ for all $j \in[\operatorname{dom} \varphi]_{t}$, or

(p) $[\operatorname{dom} \varphi]_{t}=\operatorname{dom}[\varphi]_{t}$ and $\mathcal{U}^{1}(\varphi(t))=[\varphi]_{t}\left[\mathcal{U}^{2}(t)\right]$;

(iii) $\varphi[\Sigma(\operatorname{dom} \varphi)] \subseteq \Sigma T^{1}$.

We say that $t$ is collapsing [preserving, resp.] if case (c) [(p), resp.] occurs for $t$.

An equivalent definition goes by recursion on rank of $T^{2}$.

(i) If $T^{2}=\{0\}, \varphi: \mathcal{U}^{2} \rightarrow \mathcal{U}^{1}$ iff $\mathcal{U}^{1}=0, T^{1}=\{0\}, \varphi(0)=0$.

(ii) If $T^{2}=\{0\} \cup \bigcup_{j \in J}\langle j\rangle \frown T_{\langle j\rangle}^{2}, \varphi: \mathcal{U}^{2} \rightarrow \mathcal{U}^{1}$ iff $\varphi(0)=0$, [dom $\left.\varphi\right]_{0} \in \mathcal{U}^{2}(0)$, and either

(c) for all $j \in[\operatorname{dom} \varphi]_{0}, \varphi(\langle j\rangle)=0$ and $\varphi_{\langle j\rangle}: \mathcal{U}_{\langle j\rangle}^{2} \rightarrow \mathcal{U}^{1}$ for all $j \in[\operatorname{dom} \varphi]_{0}$, or

(p) $T^{1}=\{0\} \cup \bigcup_{i \in I}\langle i\rangle \frown T_{\langle i\rangle}^{1}, \quad[\operatorname{dom} \varphi]_{0}=\operatorname{dom}[\varphi]_{0}, \quad \mathcal{U}^{1}(0)=[\varphi]_{0}\left[\mathcal{U}^{2}(0)\right]$ and $\boldsymbol{\varphi}_{\langle j\rangle}: \mathcal{U}_{\langle j\rangle}^{2} \rightarrow \mathcal{U}_{\varphi(\langle j\rangle)}^{1}=\mathcal{U}_{\left\langle\varphi_{0}(j)\right\rangle}^{1}$ for all $j \in[\operatorname{dom} \varphi]_{0}$.

Among the easy consequences of this definition are:

(1) For $t_{1}, t_{2} \in \operatorname{dom} \varphi, t_{1} \subseteq t_{2} \Rightarrow \varphi\left(t_{1}\right) \subseteq \varphi\left(t_{2}\right)$; $\operatorname{ran} \varphi$ is a subtree of $T^{1}$; and $\Sigma(\operatorname{dom} \varphi) \subseteq \Sigma T^{2}$.

(2) $I d_{T}$ is a morphism of $\mathcal{U}$ to $\mathcal{U}$.

(3) If $\varphi: \mathcal{U}^{2} \rightarrow \mathcal{U}^{1}$ and $\psi: \mathcal{U}^{1} \rightarrow \mathcal{U}^{0}$, then $\psi \circ \varphi: \mathcal{U}^{2} \rightarrow \mathcal{U}^{0}$.

(4) If $t \in \operatorname{dom} \varphi$, then $\varphi_{t}: \mathcal{U}_{t}^{2} \rightarrow \mathcal{U}_{\varphi(t)}^{1}$ is a morphism.

Proposition 6.4 If $\varphi: \mathcal{U}^{2} \rightarrow \mathcal{U}^{1}$, then $\Sigma \mathcal{U}^{1}=\varphi\left[\Sigma \mathcal{U}^{2}\right]$ 
It would be more correct to write $\Sigma \mathcal{U}^{1}=\left(\varphi \uparrow \Sigma T^{2}\right)\left[\Sigma \mathcal{U}^{2}\right]$; we take this notational license here and elsewhere.

Proof By induction on rank of $T^{2}$.

The claim is trivial when $T^{2}=\{0\}$. Assume that $T^{2}=\{0\} \cup \bigcup_{j \in J}\langle j\rangle \frown T_{j}^{2}$.

Collapsing case: By inductive assumption, $\Sigma \mathcal{U}^{1}=\varphi_{j}\left[\Sigma \mathcal{U}_{j}^{2}\right]$ for all $j \in[\operatorname{dom} \varphi]_{0}$. For $X \in \Sigma \mathcal{U}^{1}, \quad \varphi^{-1}[X]=\bigcup_{j \in[\operatorname{dom} \varphi]_{0}}\langle j\rangle \frown \varphi_{j}^{-1}[X] \in \Sigma \mathcal{U}^{2}$ because $[\operatorname{dom} \varphi]_{0} \in \mathcal{U}^{2}(0)$ and each $\varphi_{j}^{-1}[X] \in \Sigma \mathcal{U}_{j}^{2}$.

Preserving case: Then $T^{1}=\{0\} \cup \bigcup_{i \in I}\langle i\rangle \frown T_{i}^{1}$ and $\mathcal{U}_{1}(0)=[\varphi]_{0}\left[\mathcal{U}^{2}(0)\right]$. By inductive assumption, $\Sigma \mathcal{U}_{\varphi((j))}^{1}=\varphi_{j}\left[\Sigma \mathcal{U}_{j}^{2}\right]$ for all $j \in[\operatorname{dom} \varphi]_{0}$. For $X \in \Sigma \mathcal{U}^{1}$, $X^{0}:=\left\{i \in I:(X)_{i} \in \Sigma \mathcal{U}_{i}^{1}\right\} \in \mathcal{U}^{1}(0)$ and $\varphi^{-1}[X] \supseteq \bigcup\left\{\varphi_{j}^{-1}\left[(X)_{\varphi(\langle j)}\right]: j \in\right.$ $\left.[\varphi]_{0}^{-1}\left[X^{0}\right]\right\} \in \Sigma \mathcal{U}^{2}$ because $[\varphi]_{0}^{-1}\left[X^{0}\right] \in \mathcal{U}^{2}(0)$ and each $\varphi_{j}^{-1}\left[(X)_{\varphi(\langle j\rangle)}\right] \in \Sigma \mathcal{U}_{j}^{2}$.

Definition 6.5 Let $T^{\prime}, T$ be trees; the projection $\pi_{T^{\prime}, T}$ of $T$ to $T^{\prime}$ is defined as follows:

$$
\boldsymbol{\pi}_{T^{\prime}, T}(t)= \begin{cases}t & \text { if } t \in T \cap T^{\prime} \\ s & \text { if } t \in T \backslash T^{\prime}, s \in \Sigma T^{\prime}, s \subseteq t .\end{cases}
$$

We note that $\boldsymbol{\pi}_{T^{\prime}, T}$ is defined on a subset of $T$ in general, even when $T^{\prime} \subseteq T$. Also note that $T^{\prime} \cap T$ is a tree and $\boldsymbol{\pi}_{T^{\prime}, T}=\boldsymbol{\pi}_{T^{\prime} \cap T, T}$.

Lemma 6.6 Let $T^{\prime \prime} \subseteq T^{\prime} \subseteq T$ be trees. Then $\boldsymbol{\pi}_{T^{\prime \prime}, T}(t)=\boldsymbol{\pi}_{T^{\prime \prime}, T^{\prime}}\left(\boldsymbol{\pi}_{T^{\prime}, T}(t)\right)$ for all $t \in T$ where $\boldsymbol{\pi}_{T^{\prime}, T}$ is defined.

Proof Let $\pi_{T^{\prime}, T}(t)=s \in T^{\prime}$. If $t=s$, the claim is obvious. Otherwise, $s \in \Sigma T^{\prime}$. If $s \in T^{\prime \prime}$, then $s \in \Sigma T^{\prime \prime}$ and $\pi_{T^{\prime \prime}, T^{\prime}}(s)=s, t \notin T^{\prime \prime}$ and $\pi_{T^{\prime \prime}, T}(t)=s$. Otherwise, $\pi_{T^{\prime \prime}, T^{\prime}}(s)=r$ where $r \subseteq s, r \in \Sigma T^{\prime \prime}$. Then also $r \subseteq t, t \notin T^{\prime \prime}$, so $\pi_{T^{\prime \prime}, T}(t)=r$.

The projection $\boldsymbol{\pi}_{T^{\prime}, T}(t)$ may be undefined while $\boldsymbol{\pi}_{T^{\prime \prime}, T}(t)$ is defined. This is the price we pay for defining projections as partial mappings. If the obvious alternative definition of $\boldsymbol{\pi}$ as a total mapping is used, $\boldsymbol{\pi}$ may not be a morphism, according to our definition of morphism.

Definition 6.7 $T^{\prime} \subseteq T$ is a $\mathcal{U}$-subtree of $T$ (notation: $T^{\prime} \preccurlyeq \mathcal{U} T$ ) iff $\left[T^{\prime}\right]_{t} \in \mathcal{U}(t)$ for all $t \in T^{\prime} \backslash \Sigma T^{\prime}$. 
The equivalent recursive definition is:

(0) $T^{\prime} \preccurlyeq \mathcal{U}\{0\}$ iff $T^{\prime}=\{0\}$;

(1) $T^{\prime} \preccurlyeq \mathcal{U}\{0\} \cup \bigcup_{i \in I}\langle i\rangle \frown T_{i}$ iff either $T^{\prime}=\{0\}$ or $\left[T^{\prime}\right]_{0} \in \mathcal{U}(0)$ and $T_{i}^{\prime} \preccurlyeq \mathcal{U}_{i} T_{i}$ for all $i \in\left[T^{\prime}\right]_{0}$.

We say that $T^{\prime}$ is maximal iff $\Sigma T^{\prime} \subseteq \Sigma T$. If $\varphi: \mathcal{U}^{2} \rightarrow \mathcal{U}^{1}$, then $\operatorname{dom} \varphi$ is a maximal $\mathcal{U}^{2}$-subtree of $T^{2}$.

If $T^{\prime} \preccurlyeq \mathcal{U} T$, we define $\mathcal{U} \uparrow T^{\prime}=\mathcal{U}^{\prime}$ on $T^{\prime} \backslash \Sigma T^{\prime}$ by $\mathcal{U}^{\prime}(t):=\mathcal{U}(t) \uparrow\left[T^{\prime}\right]_{t}$. Clearly $\mathcal{U}^{\prime}$ is a TOU over $T^{\prime}$ and $\pi_{T^{\prime}, T}: \mathcal{U} \rightarrow \mathcal{U}^{\prime}$ is a morphism. In particular, $\Sigma\left(\mathcal{U}\left\lceil T^{\prime}\right)=\right.$ $\boldsymbol{\pi}_{T^{\prime}, T}[\Sigma \mathcal{U}]$. If $T^{\prime}$ is maximal, $\boldsymbol{\pi}_{T^{\prime}, T} \uparrow \Sigma T^{\prime}=I d_{\Sigma T^{\prime}}$ and $\Sigma\left(\mathcal{U} \uparrow T^{\prime}\right)=\Sigma \mathcal{U} \uparrow \Sigma T^{\prime}$.

Proposition 6.8 (The Factoring Lemma for TOUs)

Let $T^{\prime} \preccurlyeq \mathcal{U} T$; then $\Sigma_{\Sigma \mathcal{U} \backslash T^{\prime}}\left(\Sigma \mathcal{U}_{t}\right)$ ( $t$ ranges over $\left.\Sigma T^{\prime}\right)$ is isomorphic to $\Sigma \mathcal{U}$ via the mapping $\langle t, s\rangle \rightarrow t \frown s$.

Proof By induction on rank of $T$.

(0) If $T=\{0\}$, all is trivial [see the first part of step (1)].

(1) Let $T=\{0\} \cup \bigcup_{i \in I}\langle i\rangle \frown T_{i}$. If $T^{\prime}=\{0\}, \Sigma \mathcal{U}^{\prime}=\{\{0\}\}$, the only value of $t$ is $t=0$, and $\mathcal{U}_{0}=\mathcal{U}$. The claim is trivial.

Let $I^{\prime}:=\left[T^{\prime}\right]_{0} \in \mathcal{U}(0)$, where $T_{i}^{\prime} \preccurlyeq \mathcal{U}_{i} T_{i}$ for all $i \in I^{\prime}$. By the inductive assumption, $\Sigma \mathcal{U}_{i}$ is isomorphic to $\Sigma_{\Sigma \mathcal{U}_{i}^{\prime}}\left(\Sigma\left(\mathcal{U}_{i}\right)_{s}\right)$ where $s$ ranges over $\Sigma T_{i}^{\prime}, \mathcal{U}_{i}^{\prime}:=\mathcal{U}_{i}\left\lceil T_{i}^{\prime}=(\mathcal{U} \uparrow\right.$ $\left.T^{\prime}\right)_{i}$, and $\left(\mathcal{U}_{i}\right)_{s}=\mathcal{U}_{\langle i\rangle}$.

We note [see the definition of $\Sigma \mathcal{U}$ ] that $\Sigma\left(\mathcal{U}^{\prime}\right)=\Sigma_{\mathcal{U}(0)}\left(\Sigma \mathcal{U}_{i}^{\prime}\right)\left(i\right.$ ranges over $\left.I^{\prime}\right)$; hence $X \in \Sigma \mathcal{U} \Leftrightarrow\left\{i \in I^{\prime}:(X)_{i} \in \Sigma \mathcal{U}_{i}\right\} \in \mathcal{U}(0)$ [by definition of $\Sigma \mathcal{U}$ and $I^{\prime} \in \mathcal{U}(0)$ ] $\Leftrightarrow\left\{i \in I^{\prime}:\left\{s \in \Sigma T_{i}^{\prime}:\left(X_{i}\right)_{s} \in \Sigma\left(\mathcal{U}_{i}\right)_{s}\right\} \in \Sigma \mathcal{U}_{i}^{\prime}\right\} \in \mathcal{U}(0)$ [inductive assumption] $\Leftrightarrow\left\{i \in I^{\prime}:\left\{s \in \Sigma T_{i}^{\prime}: X_{\langle i\rangle \wedge s} \in \Sigma \mathcal{U}_{\langle i\rangle}\right\} \in \in\left(\mathcal{U} \uparrow T^{\prime}\right)_{i}\right\} \in \mathcal{U}(0)$ $\Leftrightarrow\left\{t \in \Sigma T^{\prime}: X_{t} \in \Sigma \mathcal{U}_{t}\right\} \in \Sigma \mathcal{U}^{\prime} \quad\left[\right.$ let $\langle i\rangle \frown s=t$; apply definition of $\Sigma \mathcal{U}^{\prime}$ ].

Definition 6.9 For $T^{\prime} \preccurlyeq \mathcal{U} T$ we define $\lambda_{T^{\prime}}: \subseteq \Sigma T \rightarrow \omega$ by $\lambda_{T^{\prime}}(t)=\left|\pi_{T^{\prime}, T}(t)\right|$.

We note that $\operatorname{dom} \lambda_{T^{\prime}}=\Sigma T \cap \boldsymbol{\pi}_{T^{\prime}, T}^{-1}\left[\Sigma T^{\prime}\right] \in \Sigma \mathcal{U}$.

Proposition 6.10 For every $\lambda: \subseteq \Sigma T \rightarrow \omega$ such that $\{t \in \Sigma T: \lambda(t) \leq|t|\} \in \Sigma \mathcal{U}$ there is $T^{\prime} \preccurlyeq \mathcal{U} T$ with $\lambda_{T^{\prime}}=\Sigma \mathcal{U} \lambda$.

Proof Let $T^{\prime}:=\{s \in T:(\exists t \in \Sigma T)(\exists n \leq \lambda(t))(\lambda(t) \leq|t| \wedge s=t\lceil n)$. 
Definition 6.11 For $T^{\prime}, T^{\prime \prime} \preccurlyeq \mathcal{U} T$ we let $T^{\prime \prime} \preccurlyeq \mathcal{U} T^{\prime}$ iff $\lambda_{T^{\prime \prime}} \leq_{\Sigma \mathcal{U}} \lambda_{T^{\prime}}, T^{\prime \prime} \prec_{\mathcal{U}} T^{\prime}$ iff $\lambda_{T^{\prime \prime}}<_{\Sigma \mathcal{U}} \lambda_{T^{\prime}}$, and $T^{\prime \prime} \equiv_{\mathcal{U}} T^{\prime}$ iff $\lambda_{T^{\prime \prime}}=\Sigma \mathcal{U} \lambda_{T^{\prime}}$.

Thus $T^{\prime \prime} \preccurlyeq \mathcal{U} T^{\prime}$ iff $\left\{t \in \Sigma T: \boldsymbol{\pi}_{T^{\prime \prime}, T}(t) \subseteq \boldsymbol{\pi}_{T^{\prime}, T}(t)\right\} \in \Sigma \mathcal{U}$, and similarly for $\equiv_{\mathcal{U}}$. Also, $T^{\prime} \equiv \mathcal{U} T$ iff $T^{\prime}$ is a maximal $\mathcal{U}$-subtree of $T$. Another useful observation is: $T^{\prime \prime} \preccurlyeq \mathcal{U} T^{\prime}$ iff either $T^{\prime \prime} \equiv \mathcal{U}\{0\}$ or $\left\{i \in I: T_{i}^{\prime \prime} \preccurlyeq \mathcal{U}_{i} T_{i}^{\prime}\right\} \in \mathcal{U}(0)$. It is now easy to verify that, for $T^{\prime}=T$, this definition agrees with $\preccurlyeq \mathcal{U}$ as defined in 6.7.

The relation $\preccurlyeq \mathcal{U}$ is reflexive, transitive and total. Also ( $T^{\prime \prime} \preccurlyeq \mathcal{U} T^{\prime}$ and $\left.T^{\prime} \preccurlyeq \mathcal{U} T^{\prime \prime}\right)$ iff $T^{\prime} \equiv_{\mathcal{U}} T^{\prime \prime}$, and $T^{\prime \prime} \prec_{\mathcal{U}} T^{\prime}$ iff $\left(T^{\prime \prime} \preccurlyeq \mathcal{U} T^{\prime}\right.$ and $\left.T^{\prime \prime} \not \equiv_{\mathcal{U}} T^{\prime}\right)$.

Proposition 6.12 If $T^{\prime}, T^{\prime \prime} \preccurlyeq \mathcal{U} T$, then $T^{\prime \prime} \cap T^{\prime} \preccurlyeq \mathcal{U} T$, and also $T^{\prime \prime} \preccurlyeq \mathcal{U} T^{\prime}$ iff $T^{\prime \prime} \cap T^{\prime} \equiv_{\mathcal{U}} T^{\prime \prime}$ iff $T^{\prime \prime} \cap T^{\prime} \preccurlyeq \mathcal{U} T^{\prime}$.

Proof For the first claim, $t \in\left(T^{\prime \prime} \cap T^{\prime}\right) \backslash \Sigma\left(T^{\prime \prime} \cap T^{\prime}\right)$ implies $t \notin \Sigma T^{\prime}, t \notin \Sigma T^{\prime \prime}$, so $\left[T^{\prime}\right]_{t} \in \mathcal{U}(t),\left[T^{\prime \prime}\right]_{t} \in \mathcal{U}(t)$ and $\left[T^{\prime \prime} \cap T^{\prime}\right]_{t}=\left[T^{\prime \prime}\right]_{t} \cap\left[T^{\prime}\right]_{t} \in \mathcal{U}(t)$.

Next, let $A:=\operatorname{dom} \lambda_{T^{\prime}} \cap \operatorname{dom} \lambda_{T^{\prime \prime}} \in \Sigma \mathcal{U}$. The chain of equivalences follows from the observation that, for $t \in A, \quad \lambda_{T^{\prime \prime} \cap T^{\prime}}(t)=\min \left\{\lambda_{T^{\prime}}(t), \lambda_{T^{\prime \prime}}(t)\right\}$.

Proof Let for example $\lambda_{T^{\prime \prime}}(t) \leq \lambda_{T^{\prime}}(t)$; then $s^{\prime}:=\pi_{T^{\prime}, T}(t) \in \Sigma T^{\prime}, s^{\prime \prime}:=\pi_{T^{\prime \prime}, T}(t) \in$ $\Sigma T^{\prime \prime}$ and $s^{\prime \prime} \subseteq s^{\prime}$. Hence $s^{\prime \prime} \in T^{\prime \prime} \cap T^{\prime}$, in fact, $s^{\prime \prime} \in \Sigma\left(T^{\prime \prime} \cap T^{\prime}\right)$. We conclude $s^{\prime \prime}=$ $\boldsymbol{\pi}_{T^{\prime \prime} \cap T^{\prime}, T}(t)$ and $\lambda_{T^{\prime \prime} \cap T^{\prime}}(t)=\left|s^{\prime \prime}\right|=\min \left\{\left|s^{\prime}\right|,\left|s^{\prime \prime}\right|\right\}=\min \left\{\lambda_{T^{\prime}}(t), \lambda_{T^{\prime \prime}}(t)\right\}$.

Proposition 6.13 Let $T^{\prime}, T^{\prime \prime} \preccurlyeq \mathcal{U} T$; then $T^{\prime \prime} \preccurlyeq \mathcal{U} T^{\prime}$ iff $\pi_{T^{\prime \prime}, T^{\prime}}: \mathcal{U}^{\prime} \rightarrow \mathcal{U}^{\prime \prime}$.

Proof Let $\pi:=\pi_{T^{\prime \prime}, T^{\prime}}$. If $\boldsymbol{\pi}$ is a morphism of $\mathcal{U}^{\prime}$ to $\mathcal{U}^{\prime \prime}$, then $A:=\Sigma(\operatorname{dom} \pi) \in$ $\Sigma \mathcal{U}^{\prime}$. Let $B:=\pi_{T^{\prime}, T}^{-1}[A] \in \Sigma \mathcal{U}$. By Lemma 6.6, for $t \in B, \boldsymbol{\pi}_{T^{\prime \prime}, T}(t)=\boldsymbol{\pi}\left(\boldsymbol{\pi}_{T^{\prime}, T}(t)\right)$, so $\lambda_{T^{\prime \prime}}(t)=\left|\boldsymbol{\pi}_{T^{\prime \prime}, T}(t)\right| \leq\left|\boldsymbol{\pi}_{T^{\prime}, T}(t)\right|=\lambda_{T^{\prime}}(t)$, and we conclude that $\lambda_{T^{\prime \prime}} \leq \Sigma \mathcal{U} \lambda_{T^{\prime}}$.

If $T^{\prime \prime} \preccurlyeq \mathcal{U} T^{\prime}$, we verify the requirements for $\pi$ to be a morphism. From the definition of projections, dom $\pi$ is a tree, $\pi(0)=0$, and $t \in \Sigma(\operatorname{dom} \pi)$ implies $\pi(t) \in \Sigma T^{\prime \prime}$. The key is to verify (ii). Let $t \in \operatorname{dom} \pi \backslash \Sigma T^{\prime}$. If $t \in T^{\prime \prime} \backslash \Sigma T^{\prime \prime}$, then [dom $\left.\pi\right]_{t}=$ $\left[T^{\prime \prime}\right]_{t} \cap\left[T^{\prime}\right]_{t} \in \mathcal{U}(t)$. Also $[\pi]_{t}$ is the identity on [dom $\left.\pi\right]_{t}$, so (p) holds. Otherwise, $s:=\pi(t) \in \Sigma T^{\prime \prime}$. Then $[\operatorname{dom} \pi]_{t}=\left[T^{\prime}\right]_{t} \in \mathcal{U}(t)$ and $\pi(t \frown\langle j\rangle)=s$ for all $j \in\left[T^{\prime}\right]_{t}$, so (c) holds.

Proposition 6.14 Let $\varphi: \mathcal{U}^{2} \rightarrow \mathcal{U}^{1}$ be a morphism.

(a) If $\widetilde{T}^{1} \preccurlyeq \mathcal{U}^{1} T^{1}$, then $\widetilde{T}^{2}:=\varphi^{-1}\left[\widetilde{T}^{1}\right] \preccurlyeq \mathcal{U}^{2} T^{2}$.

(b) If $\widetilde{T}^{2} \preccurlyeq \mathcal{U}^{2} T^{2}$, then $\widetilde{T}^{1}:=\varphi\left[\widetilde{T}^{2}\right] \preccurlyeq \mathcal{U}^{1} T^{1}$. 
Proof (a) Let $t \in \widetilde{T}^{2} \backslash \Sigma \widetilde{T}^{2}$; then there is $j_{0}$ such that $t \frown\left\langle j_{0}\right\rangle \in \widetilde{T}^{2} \subseteq \operatorname{dom} \varphi$. Hence $[\operatorname{dom} \varphi]_{t} \in \mathcal{U}^{2}(t)$ and either

(c), in which case $\varphi(t \frown\langle j\rangle)=\varphi(t) \in \widetilde{T}^{1}$ for all $j \in[\operatorname{dom} \varphi]_{t}$ and $[\operatorname{dom} \varphi]_{t} \subseteq\left[\widetilde{T}^{2}\right]_{t}$, so $\left[\widetilde{T}^{2}\right]_{t} \in \mathcal{U}^{2}(t)$; or

(p), in which case $[\operatorname{dom} \varphi]_{t}=\operatorname{dom}[\varphi]_{t}$ and $[\varphi]_{t}: \operatorname{dom}[\varphi]_{t} \rightarrow\left[T^{1}\right]_{\varphi(t)}$. In particular, $\varphi\left(t \frown\left\langle j_{0}\right\rangle\right)=\varphi(t) \frown\left\langle i_{0}\right\rangle \in \widetilde{T}^{1}$, hence $\varphi(t) \notin \Sigma \widetilde{T}^{1}$ and $C:=\left[\widetilde{T}^{1}\right]_{\varphi(t)} \in \mathcal{U}^{1}(t)$, $D:=[\varphi]_{t}^{-1}[C] \in \mathcal{U}^{2}(t)$, and $D \subseteq\left[\widetilde{T}^{2}\right]_{t}$, so again $\left[\widetilde{T}^{2}\right]_{t} \in \mathcal{U}^{2}(t)$.

(b) Let $s \in \widetilde{T}^{1} \backslash \Sigma \widetilde{T}^{1}$; then there is $i_{0}$ such that $s \frown\left\langle i_{0}\right\rangle \in \widetilde{T}^{1}$. Let $t^{\prime} \in \widetilde{T}^{2}$ be such that $\varphi\left(t^{\prime}\right)=s \frown\left\langle i_{0}\right\rangle$; we then can find $t, j_{0}$ such that $t \frown\left\langle j_{0}\right\rangle \subseteq t^{\prime}, \varphi(t)=s$, $\varphi\left(t \frown\left\langle j_{0}\right\rangle\right)=s \frown\left\langle i_{0}\right\rangle$. This means that $t \in \widetilde{T}^{2} \backslash \Sigma \widetilde{T}^{2}$, so $\left[\widetilde{T}^{2}\right]_{t} \in \mathcal{U}^{2}(t), t \in \operatorname{dom} \varphi$, and case (p) occurs at $t$, so $D:=[\operatorname{dom} \varphi]_{t} \cap\left[\widetilde{T}^{2}\right]_{t} \in \mathcal{U}^{2}(t)$, hence $C:=[\varphi]_{t}[D] \in \mathcal{U}^{1}(s)$ and $C \subseteq\left[\widetilde{T}^{1}\right]_{s}$. We conclude that $\left[\widetilde{T}^{1}\right]_{s} \in \mathcal{U}^{1}(s)$.

Corollary 6.15 (a) If $\widehat{T}^{1} \preccurlyeq \mathcal{U}^{1} \widetilde{T}^{1}$, then $\varphi^{-1}\left[\widehat{T}^{1}\right] \preccurlyeq \mathcal{U}^{2} \varphi^{-1}\left[\widetilde{T}^{1}\right]$.

(b) If $\widehat{T}^{1} \prec_{\mathcal{U}^{1}} \widetilde{T}^{1}$, then $\varphi^{-1}\left[\widehat{T}^{1}\right] \prec_{\mathcal{U}^{2}} \varphi^{-1}\left[\widetilde{T}^{1}\right]$.

(c) If $\widehat{T}^{2} \preccurlyeq \mathcal{U}^{2} \widetilde{T}^{2}$, then $\varphi\left[\widehat{T}^{2}\right] \preccurlyeq \mathcal{U}^{1} \varphi\left[\widetilde{T}^{2}\right]$, but (c) may fail if $\preccurlyeq$ is replaced by $\prec$.

Clearly $T^{(0)}:=\{0\} \preccurlyeq \mathcal{U} T^{\prime}$ for every $T^{\prime} \preccurlyeq \mathcal{U} T$. If $T^{\prime} \not \equiv \mathcal{U} T^{(0)}$, the function $\lambda(t):=$ $\lambda_{T^{\prime}}(t)-1$ is defined $\Sigma \mathcal{U}$-almost everywhere, and there is a $\mathcal{U}$-subtree $T_{-}^{\prime}$ of $T$ such that $\lambda_{T^{\prime}}=\Sigma \mathcal{U} \lambda$ (Proposition 6.10). We say that $T_{-}^{\prime}$ is a predecessor of $T^{\prime}$ (in the preordering $\preccurlyeq \mathcal{U})$. Indeed, $T_{-}^{\prime} \preccurlyeq \mathcal{U} T^{\prime}, T_{-}^{\prime} \not \equiv_{\mathcal{U}} T^{\prime}$, and if $T^{\prime \prime} \preccurlyeq \mathcal{U} T^{\prime}$, then either $T^{\prime \prime} \preccurlyeq \mathcal{U} T_{-}^{\prime}$ or $T^{\prime \prime} \equiv \mathcal{U} T_{-}^{\prime}$.

Analogously, if $T^{\prime} \not \neq_{\mathcal{U}} T, \lambda(t):=\lambda_{T^{\prime}}(t)+1 \leq|t|$ holds $\Sigma \mathcal{U}$-almost everywhere, and there is a $\mathcal{U}$-subtree $T_{+}^{\prime}$ of $T$ such that $\lambda_{T_{+}^{\prime}}=\Sigma \mathcal{U} \lambda$. We say that $T_{+}^{\prime}$ is a successor of $T^{\prime}$; indeed, $T^{\prime} \preccurlyeq \mathcal{U} T_{+}^{\prime}, T^{\prime} \not \mathcal{U}_{\mathcal{U}} T_{+}^{\prime}$, and if $T^{\prime} \preccurlyeq \mathcal{U} T^{\prime \prime}$ then either $T_{+}^{\prime} \preccurlyeq \mathcal{U} T^{\prime \prime}$ or $T^{\prime \prime} \equiv \mathcal{U} T^{\prime}$.

It is also clear that $\left(T_{-}^{\prime}\right)_{+} \equiv_{\mathcal{U}} T^{\prime}$ and $\left(T_{+}^{\prime}\right)_{-} \equiv_{\mathcal{U}} T^{\prime}$, as long as all these trees are defined. In particular, $T^{(1)}:=\{0\} \cup\{\langle i\rangle: i \in I\} \preccurlyeq \mathcal{U} T^{\prime}$ for every $T^{\prime} \not \equiv \mathcal{U} T^{(0)}$, and $T^{(1)} \equiv \mathcal{U} T_{+}^{(0)}$ is a successor of $T^{(0)}$. For $\mathcal{U}^{(1)}:=\mathcal{U} \uparrow T^{(1)}, \Sigma \mathcal{U}^{(1)}$ is isomorphic to $\mathcal{U}(0)$ via the map $\langle i\rangle \mapsto i$. Hence $\mathcal{U} \ell t\left(\mathbb{V} ; \Sigma \mathcal{U}^{(1)}\right)$ is naturally isomorphic to $\mathcal{U} \ell t(\mathbb{V} ; \mathcal{U}(0))$, and we sometimes identify these two interpretations in order to simplify notation.

We conclude this section with a result showing that morphisms are uniquely determined by their behavior on leaves.

Proposition 6.16 If $\varphi, \psi: \mathcal{U}^{2} \rightarrow \mathcal{U}^{1}$ and $\varphi \uparrow \Sigma T^{2}=_{\Sigma \mathcal{U}^{2}} \psi \uparrow \Sigma T^{2}$, then there is a maximal tree $\widetilde{T} \preccurlyeq \mathcal{U}^{2} T^{2}$ such that $\widetilde{T} \subseteq \operatorname{dom} \varphi \cap \operatorname{dom} \psi$ and $\varphi \uparrow \widetilde{T}=\psi \uparrow \widetilde{T}$. 
Proof By induction on the rank of $T^{2}$ it is easy to show that for every $S \in \Sigma \mathcal{U}^{2}$ there is a maximal $\widetilde{T} \preccurlyeq \mathcal{U}^{2} T^{2}$ such that $\Sigma \widetilde{T} \subseteq S$. Now let $S:=\left\{t \in \Sigma T^{2}: \varphi(t)=\psi(t)\right\}$ and consider the corresponding $\widetilde{T}$. We prove that $\varphi(s)=\psi(s)$ for all $s \in \widetilde{T}$.

Assuming the contrary, fix $s \in \widetilde{T}$ of minimal length such that $\varphi(s) \neq \psi(s)$; without loss of generality $\varphi(s) \subset \psi(s)$ [note $\varphi(s), \psi(s)$ are compatible, because $\varphi(s) \subseteq$ $\varphi(t)=\psi(t) \supseteq \psi(s)$, for some $t \in \Sigma \widetilde{T}]$. Then $s$ is collapsing for $\varphi$ and preserving for $\psi$; ie, $\varphi(s \frown\langle i\rangle)=\varphi(s)$ for all $i \in[\widetilde{T}]_{s}$, while $\mathcal{U}^{1}(\psi(s))=[\psi]_{s}\left[\mathcal{U}^{2}(s)\right]$. For every $i \in[\widetilde{T}]_{s}$ there is $s^{\prime} \supseteq s \frown\langle i\rangle$ preserving for $\varphi$ [otherwise, for $s \frown\langle i\rangle \subseteq t \in \Sigma \widetilde{T}$, $\varphi(t)=\varphi(s) \subset \psi(s) \subseteq \psi(t)]$. Fix $i_{1}, i_{2} \in[\widetilde{T}]_{s}$ such that $[\psi]_{s}\left(i_{1}\right) \neq[\psi]_{s}\left(i_{2}\right)$, and $s^{\prime} \supseteq s \frown\left\langle i_{1}\right\rangle, s^{\prime \prime} \supseteq s \frown\left\langle i_{2}\right\rangle$ of minimal length preserving for $\varphi$. Now $[\varphi]_{s^{\prime}}\left[\mathcal{U}^{2}\left(s^{\prime}\right)\right]=$ $\mathcal{U}^{1}(\varphi(s))=[\varphi]_{s^{\prime \prime}}\left[\mathcal{U}^{2}\left(s^{\prime \prime}\right)\right]$; so there are $i_{1}^{\prime}, i_{2}^{\prime}$ such that $[\varphi]_{s^{\prime}}\left(i_{1}^{\prime}\right)=[\varphi]_{s^{\prime \prime}}\left(i_{2}^{\prime}\right)=: j$; ie, $\varphi\left(s^{\prime} \frown\left\langle i_{1}^{\prime}\right\rangle\right)=\varphi\left(s^{\prime \prime} \frown\left\langle i_{2}^{\prime}\right\rangle\right)=\varphi(s) \frown\langle j\rangle$. Let $t^{\prime} \supseteq s^{\prime} \frown\left\langle i_{1}^{\prime}\right\rangle, t^{\prime \prime} \supseteq s^{\prime \prime} \frown\left\langle i_{2}^{\prime}\right\rangle$, $t^{\prime}, t^{\prime \prime} \in \Sigma \widetilde{T}$, and let $k:=|\varphi(s)|$.

On one hand, $\varphi\left(t^{\prime}\right)_{k}=\varphi\left(s^{\prime} \frown\left\langle i_{1}^{\prime}\right\rangle\right)_{k}=j=\varphi\left(s^{\prime \prime} \frown\left\langle i_{2}^{\prime}\right\rangle\right)_{k}=\varphi\left(t^{\prime \prime}\right)_{k}$. On the other hand, $\boldsymbol{\psi}\left(t^{\prime}\right)_{k}=\psi\left(s \frown\left\langle i_{1}\right\rangle\right)_{k}=[\psi]_{s}\left(i_{1}\right) \neq[\psi]_{s}\left(i_{2}\right)=\psi\left(s^{\prime \prime} \frown\left\langle i_{2}\right\rangle\right)_{k}=\psi\left(t^{\prime \prime}\right)_{k}$. But $\varphi\left(t^{\prime}\right)=\psi\left(t^{\prime}\right), \varphi\left(t^{\prime \prime}\right)=\psi\left(t^{\prime \prime}\right)$, a contradiction.

Corollary 6.17 If $\varphi: \mathcal{U} \rightarrow \mathcal{U}$, then there is a maximal tree $\widetilde{T} \preccurlyeq \mathcal{U} T$ such that $\widetilde{T} \subseteq \operatorname{dom} \varphi$ and $\varphi(t)=t$ for all $t \in \widetilde{T}$.

Proof Proposition 6.4 asserts that $\varphi \backslash \Sigma T$ is a morphism of $\Sigma \mathcal{U}$ to $\Sigma \mathcal{U}$; from this, $\varphi \uparrow \Sigma T=\Sigma \mathcal{U} I d_{T} \uparrow \Sigma T$ follows by the theorem of Katětov.

\section{Stratified Ultrapowers.}

Every TOU $\mathcal{U}$ has an associated ultrafilter $\Sigma \mathcal{U}$ over $\Sigma T_{\mathcal{U}}$, and hence an associated ultrapower $\mathcal{U} \ell(\mathbb{V} ; \Sigma \mathcal{U})$.

The universe of this interpretation is the class $\mathbb{V}^{\Sigma \mathcal{U}}$ of functions defined $\Sigma \mathcal{U}$-almost everywhere, and $=$ and $\in$ are interpreted by $=_{\Sigma \mathcal{U}}$ and $\epsilon_{\Sigma \mathcal{U}}$, resp.

If $\varphi: \mathcal{U}^{2} \rightarrow \mathcal{U}^{1}$ is a morphism of TOUs, then $\varphi \uparrow \Sigma T^{2}$ is a morphism of ultrafilters. For $f \in \mathbb{V}^{\Sigma U^{1}}$ we let $\varphi^{*}(f):=\left(\varphi \uparrow \Sigma T^{2}\right)^{*}(f)=(f \circ \varphi) \uparrow \Sigma T^{2}$; $\varphi^{*}: \mathcal{U} \ell\left(\mathbb{V} ; \Sigma \mathcal{U}^{1}\right) \rightarrow \mathcal{U} \ell\left(\mathbb{V} ; \Sigma \mathcal{U}^{2}\right)$ is a morphism.

If now $T^{\prime} \preccurlyeq \mathcal{U} T$ and $\mathcal{U}^{\prime}:=\mathcal{U} \uparrow T^{\prime}$, the projection $\pi_{T^{\prime}, T}$ is a morphism of $\Sigma \mathcal{U}$ onto $\Sigma \mathcal{U}^{\prime}$, and hence induces an elementary embedding $\pi_{T^{\prime}, T}^{*}$ of $\mathcal{U} \ell\left(\mathbb{V} ; \Sigma \mathcal{U}^{\prime}\right)$ into $\mathcal{U} \ell(\mathbb{V} ; \Sigma \mathcal{U})$, defined by $\boldsymbol{\pi}_{T^{\prime}, T}^{*}(f)=\left(f \circ \boldsymbol{\pi}_{T^{\prime}, T}\right) \uparrow \Sigma T$, for $f \in \mathbb{V}^{\Sigma \mathcal{U}^{\prime}}$. More than that; 
for $u:=\left\langle\Sigma \mathcal{U}_{t}: t \in \Sigma T^{\prime}\right\rangle, \mathcal{U} \ell\left(\mathbb{V} ; \Sigma \mathcal{U}^{\prime}\right) \vDash$ " $u$ is an ultrafilter", and, by Propositions 4.1 and 6.8, $\left(\mathcal{U} \ell(\mathbb{V} ; \Sigma \mathcal{U}), \pi_{T^{\prime}, T}^{*}\left[\mathbb{V}^{\Sigma \mathcal{U}^{\prime}}\right]\right)$ is isomorphic to $[\mathcal{U} \ell t(\mathbb{V} ; u)]^{\mathcal{U} \ell\left(\mathbb{V} ; \Sigma \mathcal{U}^{\prime}\right)}$. It follows that $\left(\mathcal{U} \ell(\mathbb{V} ; \Sigma \mathcal{U}), \pi_{T^{\prime}, T}^{*}\left[\mathbb{V}^{\left.\left.\Sigma \mathcal{U}^{\prime}\right]\right)}\right.\right.$ is an interpretation of a nonstandard set theory satisfying all the axioms of ST. In particular we note that there are many ways in which the standardness predicate can be interpreted so that ST holds, one for each $T^{\prime} \preccurlyeq \mathcal{U} T$; the universe of $\mathcal{U} \ell(\mathbb{V} ; \Sigma \mathcal{U})$ is stratified into multiple levels of standardness. We use a binary predicate $\sqsubseteq_{\mathcal{U}}$ to describe this stratification.

To simplify the notation, we "identify" $f \in \mathbb{V}^{\Sigma \mathcal{U}^{\prime}}$ with $\pi_{T^{\prime}, T}^{*}(f) \in \mathbb{V}^{\Sigma \mathcal{U}}$ when there is (hopefully) no danger of misunderstanding. This allows us to regard $\mathbb{V}^{\Sigma \mathcal{U}^{\prime}}$ as a subclass of $\mathbb{V}^{\Sigma \mathcal{U}}$ : for $g \in \mathbb{V}^{\Sigma \mathcal{U}}$, the statement " $g \in \mathbb{V}^{\Sigma \mathcal{U}^{\prime}}$ " is to be interpreted as "there exists $h \in \mathbb{V}^{\Sigma \mathcal{U}^{\prime}}$ such that $\pi_{T^{\prime}, T}^{*}(h)=\Sigma \mathcal{U} g$ ".

Definition 7.1 For $f, g \in \mathbb{V}^{\Sigma \mathcal{U}}$, we define:

$$
f \sqsubseteq \mathcal{U} g \quad \text { iff } \quad\left(\forall T^{\prime} \preccurlyeq \mathcal{U} T\right)\left[g \in \mathbb{V}^{\Sigma \mathcal{U}^{\prime}} \Rightarrow f \in \mathbb{V}^{\Sigma \mathcal{U}^{\prime}}\right] .
$$

It is clear that $\sqsubseteq_{\mathcal{U}}$ is reflexive and transitive. It is also total: $\left(\forall f, g \in \mathbb{V}^{\Sigma \mathcal{U}}\right)(f \sqsubseteq \mathcal{U}$ $g \vee g \sqsubseteq \mathcal{U} f)$.

Proof Suppose that neither $f \sqsubseteq \mathcal{U} g$ nor $g \sqsubseteq_{\mathcal{U}} f$ holds. Then there is $T^{\prime} \preccurlyeq \mathcal{U} T$ such that $g \in \mathbb{V}^{\Sigma \mathcal{U}^{\prime}} \wedge f \notin \mathbb{V}^{\Sigma \mathcal{U}^{\prime}}$, and also $T^{\prime \prime} \preccurlyeq \mathcal{U} T$ such that $f \in \mathbb{V}^{\Sigma \mathcal{U}^{\prime \prime}} \wedge g \notin \mathbb{V}^{\Sigma \mathcal{U}^{\prime \prime}}$. Either $T^{\prime} \preccurlyeq \mathcal{U} T^{\prime \prime}$ or $T^{\prime \prime} \preccurlyeq \mathcal{U} T^{\prime}$. In the first case, $\pi_{T^{\prime}, T^{\prime \prime}}^{*}(g) \in \mathbb{V}^{\Sigma \mathcal{U}^{\prime \prime}}$, and hence (Lemma 6.6 and Proposition 6.13) $\boldsymbol{\pi}_{T^{\prime}, T}^{*}(g)=\Sigma \mathcal{U} \boldsymbol{\pi}_{T^{\prime \prime}, T}^{*}\left(\boldsymbol{\pi}_{T^{\prime}, T^{\prime \prime}}^{*}(g)\right) \in \mathbb{V}^{\Sigma \mathcal{U}^{\prime \prime}}$, a contradiction. The second case is similar.

Interpretations $(\mathcal{U} \ell(\mathbb{V} ; \Sigma \mathcal{U}), \sqsubseteq \mathcal{U})$, that is, $\left(\mathbb{V}^{\Sigma \mathcal{U}},=_{\Sigma \mathcal{U}}, \in_{\Sigma \mathcal{U}}, \sqsubseteq \Sigma \mathcal{U}\right)$, for the $\{\in, \sqsubseteq\}-$ language will be called stratified ultrapowers of the universe and denoted $\mathcal{U} \ell t(\mathbb{V} ; \mathcal{U})$.

Proposition 7.2 For every $f \in \mathbb{V}^{\Sigma \mathcal{U}}$ there is a unique (modulo $\left.\equiv \mathcal{U}\right)$ tree $T(f) \preccurlyeq \mathcal{U} T$ such that, for all $T^{\prime} \preccurlyeq \mathcal{U} T, f \in \mathbb{V}^{\Sigma \mathcal{U}^{\prime}} \Leftrightarrow T(f) \preccurlyeq \mathcal{U} T^{\prime}$.

Proof By induction on rank of $T$.

(0) If $T=T^{(0)}, T(f):=T^{(0)}$.

(1) If $T=\{0\} \cup \bigcup_{i \in I}\langle i\rangle \frown T_{i}$, we distinguish two cases.

(a) $f=\Sigma \mathcal{U} \mathfrak{k}_{\Sigma \mathcal{U}}(c)$ for some $c \in \mathbb{V}$. Then $T(f):=T^{(0)}$ again clearly works. 
(b) Otherwise, we let $I_{0}:=\left\{i \in I: \operatorname{dom} f_{i} \in \Sigma \mathcal{U}_{i}\right\}$ (note $\left.I_{0} \in \mathcal{U}(0)\right)$ and use the inductive assumption to choose, for each $i \in I_{0}$, a particular $T\left(f_{i}\right) \preccurlyeq \mathcal{U}_{i} T_{i}$. We let $T(f):=\{0\} \cup \bigcup_{i \in I_{0}}\langle i\rangle \frown T\left(f_{i}\right)$; clearly $T(f) \preccurlyeq \mathcal{U} T$ and $f \in \mathbb{V}^{\Sigma \mathcal{U}_{T(f)}}$.

Assume $f \in \mathbb{V}^{\Sigma \mathcal{U}^{\prime}}$ for some $T^{\prime} \preccurlyeq \mathcal{U} T$. Then $T^{(0)} \prec_{\mathcal{U}} T^{\prime}$ because $f \neq \neq_{\Sigma \mathcal{U}} \mathfrak{k}_{\Sigma \mathcal{U}}(c)$ for any $c$, and $I^{\prime}:=\left\{i \in I_{0}: f_{i} \in \mathbb{V}^{\Sigma \mathcal{U}_{i}^{\prime}}\right\} \in \mathcal{U}(0)$. By the inductive assumption, $T\left(f_{i}\right) \preccurlyeq \mathcal{U}_{i} T_{i}^{\prime}$ for $i \in I^{\prime}$. Hence $T(f) \preccurlyeq \mathcal{U} T^{\prime}$.

Conversely, assume $T(f) \preccurlyeq \mathcal{U} T^{\prime} \preccurlyeq \mathcal{U} T$. Then $T^{(0)} \prec_{\mathcal{U}} T^{\prime}$ and $I^{\prime}:=\left\{i \in I_{0}:\right.$ $\left.T\left(f_{i}\right) \preccurlyeq \mathcal{U}_{i} T_{i}^{\prime}\right\} \in \mathcal{U}(0)$. By the inductive assumption, $f_{i} \in \mathbb{V}^{\Sigma \mathcal{U}_{i}^{\prime}}$ for all $i \in I^{\prime}$, and hence $f \in \mathbb{V}^{\Sigma \mathcal{U}^{\prime}}$.

Corollary 7.3 (a) $f \sqsubseteq \mathcal{U} g \Leftrightarrow T(f) \preccurlyeq \mathcal{U} T(g)$, for all $f, g \in \mathbb{V}^{\Sigma \mathcal{U}}$.

(b) Let $D_{T^{\prime}}(t)=t$ for all $t \in \Sigma T^{\prime}$, where $T^{\prime} \preccurlyeq \mathcal{U} T$; then $T\left(D_{T^{\prime}}\right) \equiv \mathcal{U} T^{\prime}$.

(c) Let $E_{T^{\prime}}(t)=t_{k}$ for all $t=\left\langle t_{0}, \ldots, t_{k}\right\rangle \in \Sigma T^{\prime}$, where $T^{(0)} \prec_{\mathcal{U}} T^{\prime} \preccurlyeq \mathcal{U} T$; then $T\left(E_{T^{\prime}}\right) \equiv \mathcal{U} T^{\prime}$.

Proposition 7.4 Let $\varphi: \mathcal{U}^{2} \rightarrow \mathcal{U}^{1}$; then $T\left(\varphi^{*}(f)\right) \equiv_{\mathcal{U}^{2}}\left(\varphi^{-1}\left[(T(f))_{-}\right]\right)_{+}$, for any $f \in \mathbb{V}^{\Sigma \mathcal{U}^{1}}$ such that $T^{(0)} \prec_{\mathcal{U}^{1}} T(f)$.

Proof Let $f \in \mathbb{V}^{\Sigma \mathcal{U}^{1}}, T^{(0)} \prec_{\mathcal{U}^{1}} T(f)$, and $T(f) \preccurlyeq \mathcal{U}^{1} T^{\prime}$. Let $\mathcal{U}(f):=\mathcal{U} \uparrow T(f)$. The fact that $f \in \mathbb{V}^{\Sigma \mathcal{U}(f)}$ means that there is a set $X \in \Sigma \mathcal{U}^{1}$ such that, for $t^{\prime}, t^{\prime \prime} \in X$, $\boldsymbol{\pi}_{T(f), T^{\prime}}\left(t^{\prime}\right)=\boldsymbol{\pi}_{T(f), T^{\prime}}\left(t^{\prime \prime}\right) \Rightarrow f\left(t^{\prime}\right)=f\left(t^{\prime \prime}\right)$. Let $\widetilde{T}:=\left(\varphi^{-1}\left[(T(f))_{-}\right]\right)_{+}$and $\widetilde{\mathcal{U}}:=$ $\mathcal{U} \uparrow \widetilde{T}$. We note that $T(f)_{-} \prec_{\mathcal{U}^{1}} T(f)$, so $\varphi^{-1}\left[T(f)_{-}\right] \prec_{\mathcal{U}^{2}} \varphi^{-1}[T(f)], \widetilde{T} \preccurlyeq \mathcal{U}^{2}$ $\varphi^{-1}[T(f)]$ is defined, and $Z:=\left\{s \in \Sigma T^{2}: \lambda_{\varphi^{-1}\left[(T(f))_{-}\right]}<\lambda_{\varphi^{-1}[(T(f))]}\right\} \in \Sigma \mathcal{U}^{2}$. Let $Y:=\varphi^{-1}[X] \cap \pi_{\widetilde{T}, T^{2}}^{-1}[\Sigma \widetilde{T}] \cap Z$; then $Y \in \Sigma \mathcal{U}^{2}$. If $s^{\prime}, s^{\prime \prime} \in Y$ and $\widetilde{s}:=\pi_{\widetilde{T}, T^{2}}\left(s^{\prime}\right)=$ $\pi_{\widetilde{T}, T^{2}}\left(s^{\prime \prime}\right)$, then $\widetilde{s}=s \frown\langle j\rangle$ for some $s \in \varphi^{-1}\left[(T(f))_{-}\right]$. Hence $\varphi(s) \in(T(f))_{-}$and $\varphi(\widetilde{s}) \in T(f)$. Also $\varphi(\widetilde{s})=\pi_{T(f), T^{\prime}}\left(\varphi\left(s^{\prime}\right)\right)=\pi_{T(f), T^{\prime}}\left(\varphi\left(s^{\prime \prime}\right)\right)$. As $\varphi\left(s^{\prime}\right), \varphi\left(s^{\prime \prime}\right) \in X$, we have $f\left(\varphi\left(s^{\prime}\right)\right)=f\left(\varphi\left(s^{\prime \prime}\right)\right)$. This proves that $\varphi^{*}(f)=(f \circ \varphi) \uparrow \Sigma T^{2} \in \mathbb{V}^{\Sigma \tilde{\mathcal{U}}}$, ie, $T\left(\varphi^{*}(f)\right) \preccurlyeq \mathcal{U}^{2} \widetilde{T}$.

Now assume that $T\left(\varphi^{*}(f)\right) \prec_{\mathcal{U}^{2}} \widetilde{T}$; then $T\left(\varphi^{*}(f)\right) \preccurlyeq \mathcal{U}^{2} \widetilde{T}_{-}=\varphi^{-1}\left[(T(f))_{-}\right]$. Hence there is a set $Y \in \Sigma \mathcal{U}^{2}, \quad Y \subseteq \pi_{\widetilde{T}_{-}, T^{2}}^{-1}\left[\Sigma \widetilde{T}_{-}\right]$, such that $t^{\prime}, t^{\prime \prime} \in Y, \quad \pi_{\widetilde{T}_{-}, T^{2}}\left(t^{\prime}\right)=$ $\boldsymbol{\pi}_{\widetilde{T}_{-}, T^{2}}\left(t^{\prime \prime}\right) \Rightarrow f\left(\varphi\left(t^{\prime}\right)\right)=f\left(\varphi\left(t^{\prime \prime}\right)\right)$. By the Factoring Lemma for TOUs, there is $\widetilde{Y} \in \Sigma\left(\mathcal{U}^{2} \uparrow \widetilde{T}_{-}\right)$and for every $\widetilde{t} \in \widetilde{Y}$ a set $Y_{\widetilde{t}} \in \Sigma \mathcal{U}_{\widetilde{t}}$ such that $\bigcup_{\tilde{t} \in \widetilde{Y}} \widetilde{t} \frown Y_{\widetilde{t}} \subseteq Y$. [We note in particular that $v^{\prime}, v^{\prime \prime} \in Y_{\widetilde{t}} \Rightarrow f\left(\varphi\left(\widetilde{t} \frown v^{\prime}\right)\right)=f\left(\varphi\left(\widetilde{t} \frown v^{\prime \prime}\right)\right)$.] The set $\widetilde{X}:=\varphi[\widetilde{Y}] \in \Sigma\left(\mathcal{U}^{1}\left\lceil T(f)_{-}\right)\right.$. For every $\widetilde{s} \in \widetilde{X}$ pick one $\widetilde{t} \in \widetilde{Y}$ such that $\widetilde{s}=\varphi(\widetilde{t})$, and let $X_{\widetilde{s}}:=\varphi_{\tilde{t}}\left[Y_{\tilde{t}}\right] ; \quad X_{\widetilde{s}} \in \Sigma \mathcal{U}_{\tilde{s}}^{1}$. Hence $X:=\bigcup_{\widetilde{s} \in \widetilde{X}} \widetilde{s} \frown X_{\widetilde{s}} \in \Sigma \mathcal{U}^{1}$. Let $\widetilde{s} \frown u^{\prime}, \widetilde{s} \frown u^{\prime \prime} \in X$. Fix $v^{\prime}, v^{\prime \prime} \in Y_{\widetilde{t}}$ such that $u^{\prime}=\varphi_{\widetilde{t}}\left(v^{\prime}\right), u^{\prime \prime}=\varphi_{\widetilde{t}}\left(v^{\prime \prime}\right)$. Then 
$\widetilde{s} \frown u^{\prime}=\varphi\left(\widetilde{t} \frown v^{\prime}\right), \widetilde{s} \frown u^{\prime \prime}=\varphi\left(\widetilde{t} \frown v^{\prime \prime}\right)$ and $f\left(\widetilde{s} \frown u^{\prime}\right)=f\left(\varphi\left(\widetilde{t} \frown v^{\prime}\right)\right)=f(\varphi(\widetilde{t} \frown$ $\left.\left.v^{\prime \prime}\right)\right)=f\left(\widetilde{s} \frown u^{\prime \prime}\right)$. Hence $f \in \mathbb{V}^{\Sigma\left(\mathcal{U}^{1} \mid T(f)_{-}\right)}$, ie, $T(f) \preccurlyeq \mathcal{U}^{1} T(f)_{-}$, a contradiction.

Definition 7.5 $\Phi: \mathbb{V}^{\Sigma \mathcal{U}^{1}} \rightarrow \mathbb{V}^{\Sigma \mathcal{U}^{2}}$ is a premorphism of $\mathcal{U} \ell t\left(\mathbb{V} ; \mathcal{U}^{1}\right)$ to $\mathcal{U} \ell t\left(\mathbb{V} ; \mathcal{U}^{2}\right)$ iff $\Phi$ is a morphism of $\mathcal{U} \ell\left(\mathbb{V} ; \Sigma \mathcal{U}^{1}\right)$ to $\mathcal{U} \ell\left(\mathbb{V} ; \Sigma \mathcal{U}^{2}\right)$ and $\Phi$ preserves $\sqsubseteq$, ie, $f \sqsubseteq_{\mathcal{U}^{1}} g \Leftrightarrow \Phi(f) \sqsubseteq_{\mathcal{U}^{2}} \Phi(g)$ holds for all $f, g \in \mathbb{V}^{\Sigma \mathcal{U}^{1}}$.

Proposition 7.6 If $\varphi: \mathcal{U}^{2} \rightarrow \mathcal{U}^{1}$, then $\varphi^{*}$ is a premorphism of $\mathcal{U} \ell t\left(\mathbb{V} ; \mathcal{U}^{1}\right)$ to $\mathcal{U} \ell t\left(\mathbb{V} ; \mathcal{U}^{2}\right)$.

Proof Propositions 6.4 and 2.4 show that $\varphi^{*}$ is a morphism of $\mathcal{U} \ell\left(\mathbb{V} ; \Sigma \mathcal{U}^{1}\right)$ to $\mathcal{U} \ell\left(\mathbb{V} ; \Sigma \mathcal{U}^{2}\right)$; we have to prove that $\varphi^{*}$ preserves $\sqsubseteq$.

If $T(f) \equiv_{\mathcal{U}^{1}} T^{(0)}$, then $f={ }_{\Sigma \mathcal{U}^{1}} \mathfrak{k}_{1}(c)$ for some $c \in \mathbb{V}$, so $\varphi^{*}(f)=_{\Sigma \mathcal{U}^{2}} \mathfrak{k}_{2}(c)$ and $T\left(\varphi^{*}(f)\right) \equiv_{\mathcal{U}^{2}} T^{(0)}$. Both $f \sqsubseteq \mathcal{U}_{1} g$ and $\varphi^{*}(f) \sqsubseteq \mathcal{U}_{2} \varphi^{*}(g)$ hold for all $g$.

So let us assume that $T^{(0)} \prec_{\mathcal{U}^{1}} T(f)$. If $f \sqsubseteq \mathcal{U}_{1} g$, then $T(f) \preccurlyeq \mathcal{U}_{1} T(g)$, and $T^{(0)} \prec_{\mathcal{U}^{1}}$ $T(g)$; we get $T(f)_{-} \preccurlyeq \mathcal{U}_{1} T(g)_{-}$; hence, using Corollary 6.15(a), $\varphi^{-1}\left[T(f)_{-}\right] \preccurlyeq \mathcal{U}_{2}$ $\varphi^{-1}\left[T(g)_{-}\right]$; and, applying + and Proposition 7.4 on both sides, finally $T\left(\varphi^{*}(f)\right) \preccurlyeq \mathcal{U}^{2}$ $T\left(\varphi^{*}(g)\right)$, ie, $\varphi^{*}(f) \sqsubseteq \mathcal{U}^{2} \varphi^{*}(g)$.

The same calculation shows that $f \sqsubset_{\mathcal{U}_{1}} g$ implies $\varphi^{*}(f) \sqsubset_{\mathcal{U}^{2}} \varphi^{*}(g)$ (use Corollary 6.15(b) in place of (a)) and completes the proof.

We already used the Factoring Lemma, Proposition 4.1, as a motivation for the introduction of $\sqsubseteq$. We now want to show that factoring preserves $\sqsubseteq$. First some notation.

Let $\mathcal{U}$ be a TOU over $T$ and let $T^{\prime} \preccurlyeq \mathcal{U} T$. We define $T / T^{\prime}$ and $\mathcal{U} / T^{\prime}: \Sigma T^{\prime} \rightarrow \mathbb{V}$ by $\left(T / T^{\prime}\right)(t):=T_{t}, \quad\left(\mathcal{U} / T^{\prime}\right)(t):=\mathcal{U}_{t}$, and note that $\mathcal{U} \ell\left(\mathbb{V} ; \Sigma \mathcal{U}^{\prime}\right) \vDash " T / T^{\prime}$ is a tree and $\mathcal{U} / T^{\prime}$ is a TOU over $T / T^{\prime \prime}$. [Recall that terms with the leading symbol / are evaluated in the ambient set theory, and need not be underlined.] For $f: \subseteq \Sigma T \rightarrow \mathbb{V}$ we define $f / T^{\prime}: \Sigma T^{\prime} \rightarrow \mathbb{V}$ by $\left(f / T^{\prime}\right)(t)=f_{t}$. [Recall that $f_{t}$ is defined by $f_{t}(s)=f(t \frown s)$; we have $f_{t}: \subseteq \Sigma T_{t} \rightarrow \mathbb{V}$ here.] Finally, we let $\Omega(f):=\Omega_{T^{\prime}, \mathcal{U}}(f):=f / T^{\prime}$, for $f \in \mathbb{V}^{\Sigma \mathcal{U}}$.

Proposition 7.7 (The Factoring Lemma) $\Omega$ is an isomorphism between $\left(\mathcal{U} \ell(\mathbb{V} ; \Sigma \mathcal{U}), \mathbb{V}^{\Sigma \mathcal{U}^{\prime}}\right)=\left(\mathbb{V}^{\Sigma \mathcal{U}},=_{\Sigma \mathcal{U}}, \in_{\Sigma \mathcal{U}}, \boldsymbol{\pi}_{T^{\prime}, T}^{*}\left[\mathbb{V}^{\Sigma \mathcal{U}^{\prime}}\right]\right)$ and $\mathcal{U} \ell\left(\left\langle\mathcal{U} \ell t\left(\mathbb{V} ; \mathcal{U}_{t}\right): t \in \Sigma T^{\prime}\right\rangle, \Sigma \mathcal{U}^{\prime}\right)=\left[\mathcal{U} \ell t\left(\mathbb{V} ; \mathcal{U} / T^{\prime}\right)\right]^{\mathcal{U} \ell\left(\mathbb{V} ; \Sigma \mathcal{U}^{\prime}\right)}$. Moreover, $\mathcal{U} \ell\left(\mathbb{V} ; \Sigma \mathcal{U}^{\prime}\right) \vDash “ \Omega\left(\pi_{T^{\prime}, T}(g)\right)=_{\Sigma\left(\mathcal{U} / T^{\prime}\right)} \mathfrak{k}_{\Sigma\left(\mathcal{U} / T^{\prime}\right)}(g)$ ” for all $g \in \mathbb{V}^{\Sigma \mathcal{U}^{\prime}}$. 
We stress that here $\mathfrak{k}_{\Sigma\left(\mathcal{U} / T^{\prime}\right)}$ is to be evaluated in $\mathcal{U} \ell\left(\mathbb{V} ; \Sigma \mathcal{U}^{\prime}\right)$, ie, $\mathcal{U} \ell\left(\mathbb{V} ; \Sigma \mathcal{U}^{\prime}\right) \vDash$ "k $\Sigma\left(\mathcal{U} / T^{\prime}\right)(g)$ is a constant function on $\Sigma\left(T / T^{\prime}\right)$ with value $g$ ", ie, if $\mathcal{U} \ell\left(\mathbb{V} ; \Sigma \mathcal{U}^{\prime}\right) \vDash$ $\mathfrak{k}_{\Sigma\left(\mathcal{U} / T^{\prime}\right)}(g)=f$, then $\left\{t \in \Sigma T^{\prime}: f(t)(s)=g(t)\right.$ for all $\left.s \in \Sigma\left(T_{t}\right)\right\} \in \Sigma \mathcal{U}^{\prime}$. We summarize the "Moreover" part by saying that " $\Omega$ is the identity on $\mathbb{V}^{\Sigma \mathcal{U}}$ ".

Proof This is Proposition 4.1 for $U=\Sigma \mathcal{U}^{\prime}, I=\Sigma T^{\prime}, U_{i}=\Sigma \mathcal{U}_{t}$ for $i=t \in I$.

The isomorphism $\Omega$ in the Factoring Lemma takes $\mathbb{V}^{\Sigma \mathcal{U}^{\prime}}$, the "coarsest level of standardness" of the ultrapower $\mathcal{U} \ell(\mathbb{V} ; \Sigma \mathcal{U})$, onto the "coarsest level of standardness" of $\left[\mathcal{U} \ell t\left(\mathbb{V} ; \Sigma\left(\mathcal{U} / T^{\prime}\right)\right)\right]^{\mathcal{U} \ell\left(\mathbb{V} ; \Sigma \mathcal{U}^{\prime}\right)}$. Our next task is to show that the ordering $\sqsubseteq$ of the "finer levels of standardness" is also preserved by $\Omega$.

For $T^{\prime} \preccurlyeq \mathcal{U} T^{\prime \prime} \preccurlyeq \mathcal{U} T$ we let $T^{\prime \prime} / T^{\prime}: \subseteq \Sigma T^{\prime} \rightarrow \mathbb{V}$ be defined by $\left(T^{\prime \prime} / T^{\prime}\right)(t)=T_{t}^{\prime \prime}$, whenever $t \in T^{\prime \prime}$.

Proposition 7.8 $\mathcal{U} \ell\left(\mathbb{V} ; \Sigma \mathcal{U}^{\prime}\right) \vDash T^{\prime \prime} / T^{\prime} \preccurlyeq \mathcal{U} / T^{\prime} T / T^{\prime}$. If also $T^{\prime} \preccurlyeq \mathcal{U} T^{\prime \prime \prime} \preccurlyeq \mathcal{U} T$, then $T^{\prime \prime} \preccurlyeq \mathcal{U} T^{\prime \prime \prime}$ iff $\mathcal{U} \ell\left(\mathbb{V} ; \Sigma \mathcal{U}^{\prime}\right) \vDash T^{\prime \prime} / T^{\prime} \preccurlyeq \mathcal{U} / T^{\prime} T^{\prime \prime \prime} / T^{\prime}$.

Proof These are easy consequences of the definition of $\preccurlyeq \mathcal{U}$, Łoś Theorem, and the Factoring Lemma for TOUs, Proposition 6.8.

Proposition 7.9 For $f \notin \mathbb{V}^{\Sigma \mathcal{U}^{\prime}}, \quad \mathcal{U} \ell\left(\mathbb{V} ; \Sigma \mathcal{U}^{\prime}\right) \vDash T\left(f / T^{\prime}\right) \equiv_{\mathcal{U} / T^{\prime}} T(f) / T^{\prime}$.

Proof Let $T^{\prime \prime}:=T(f)$; we have $T^{\prime} \preccurlyeq \mathcal{U} T^{\prime \prime}$. Also, $f=\Sigma \mathcal{U} \pi_{T^{\prime \prime}, T}^{*}(g)$ for some $g: \subseteq \Sigma T^{\prime \prime} \rightarrow \mathbb{V}$. Then $\left\{t \in \Sigma T^{\prime}: f_{t}=\Sigma \mathcal{U}_{t} \pi_{T_{t}^{\prime \prime}, T_{t}}^{*}\left(g_{t}\right)\right\} \in \Sigma \mathcal{U}^{\prime}$ and $\mathcal{U} \ell\left(\mathbb{V} ; \Sigma \mathcal{U}^{\prime}\right) \vDash$ " $f / T^{\prime}=\mathcal{U} / T^{\prime} \boldsymbol{\pi}_{T^{\prime \prime} / T^{\prime}, T / T^{\prime}}\left(g / T^{\prime}\right)$ ". (Here $*$ is evaluated in $\mathcal{U} \ell\left(\mathbb{V} ; \Sigma \mathcal{U}^{\prime}\right)$.) We conclude that $\mathcal{U} \ell\left(\mathbb{V} ; \Sigma \mathcal{U}^{\prime}\right) \vDash T\left(f / T^{\prime}\right) \preccurlyeq \mathcal{U} / T^{\prime} T(f) / T^{\prime}$.

Conversely, assume $\mathcal{U} \ell\left(\mathbb{V} ; \Sigma \mathcal{U}^{\prime}\right) \vDash ' f / T^{\prime} \in \mathbb{V}^{\tau} \wedge \tau \preccurlyeq \mathcal{U} / T^{\prime} T / T^{\prime}$ ". Then $L:=\{t \in$ $\left.\Sigma T^{\prime}: \tau(t) \preccurlyeq \mathcal{U}_{t} T_{t}\right\} \in \Sigma \mathcal{U}^{\prime}$; we let $T^{\prime \prime}:=(L \downarrow) \cup \bigcup_{t \in L}\{t \frown s: s \in \tau(t)\}$ and note that $T^{\prime \prime}$ is a tree, $T^{\prime} \preccurlyeq \mathcal{U} T^{\prime \prime} \preccurlyeq \mathcal{U} T$, and $\mathcal{U} \ell\left(\mathbb{V} ; \Sigma \mathcal{U}^{\prime}\right) \vDash$ " $T^{\prime \prime} / T^{\prime}=\tau$ ". It follows easily that $f \in \mathbb{V}^{\prime \prime}$, hence $T(f) \preccurlyeq \mathcal{U} T^{\prime \prime}$ and $\mathcal{U} \ell\left(\mathbb{V} ; \Sigma \mathcal{U}^{\prime}\right) \vDash$ “ $T(f) / T^{\prime} \preccurlyeq \mathcal{U} / T^{\prime} T^{\prime \prime} / T^{\prime}=\tau$ ".

Corollary 7.10 For $f, g \notin \mathbb{V}^{\Sigma \mathcal{U}^{\prime}}, f \sqsubseteq \mathcal{U} g$ iff $\mathcal{U} \ell\left(\mathbb{V} ; \Sigma \mathcal{U}^{\prime}\right) \vDash \Omega(f) \sqsubseteq \mathcal{U} / T^{\prime} \Omega(g)$.

Proof $f \sqsubseteq \mathcal{U} g$ iff $T(f) \preccurlyeq \mathcal{U} T(g)$ iff $\mathcal{U} \ell\left(\mathbb{V} ; \Sigma \mathcal{U}^{\prime}\right) \vDash T(f) / T^{\prime} \preccurlyeq \mathcal{U} / T^{\prime} T(g) / T^{\prime}$ iff $\mathcal{U} \ell\left(\mathbb{V} ; \Sigma \mathcal{U}^{\prime}\right) \vDash T\left(f / T^{\prime}\right) \preccurlyeq \mathcal{U} / T^{\prime} T\left(g / T^{\prime}\right)$ iff $\mathcal{U} \ell\left(\mathbb{V} ; \Sigma \mathcal{U}^{\prime}\right) \vDash \Omega(f) \sqsubseteq_{\mathcal{U}} / T^{\prime} \Omega(g)$. 
In order to summarize these results in a compact form we define a relativized version of $\sqsubseteq$ :

$$
f \sqsubseteq \mathcal{U} ; h g \quad \text { iff } f \sqsubseteq \mathcal{U} h \vee f \sqsubseteq \mathcal{U} g, \quad \text { for } f, g, h \in \mathbb{V}^{\Sigma \mathcal{U}} .
$$

The Factoring Lemma and Corollary 7.10 combine into the following key theorem.

Theorem 7.11 (The Factoring Theorem)

$\Omega_{T^{\prime}, \mathcal{U}}$ is an isomorphism between $\left(\mathcal{U} \ell(\mathbb{V} ; \Sigma \mathcal{U}), \sqsubseteq \mathcal{U} ; D_{T^{\prime}}\right)$ and

$\mathcal{U} \ell\left(\left\langle\left(\mathcal{U} \ell\left(\mathbb{V} ; \Sigma \mathcal{U}_{t}\right), \sqsubseteq \mathcal{U}_{t}\right): t \in \Sigma T^{\prime}\right\rangle, \Sigma \mathcal{U}^{\prime}\right)=\left[\mathcal{U} \ell t\left(\mathbb{V} ; \mathcal{U} / T^{\prime}\right)\right]^{\mathcal{U} \ell\left(\mathbb{V} ; \Sigma \mathcal{U}^{\prime}\right)}$

which is the identity on $\mathbb{V}^{\Sigma \mathcal{U}^{\prime}}$.

Finally, we show that factoring commutes with $\varphi^{*}$.

Theorem 7.12 (The Factoring Theorem for Morphisms)

Given $\varphi: \mathcal{U}^{2} \rightarrow \mathcal{U}^{1}$ and $\widetilde{T}^{2} \preccurlyeq \mathcal{U}^{2} T^{2}$, let $\widetilde{\mathcal{U}}^{2}:=\mathcal{U}^{2} \uparrow \widetilde{T}^{2}, \widetilde{T}^{1}:=\varphi\left[\widetilde{T}^{2}\right]$,

$\widetilde{\mathcal{U}}^{1}:=\mathcal{U}^{1} \uparrow \widetilde{T}^{1}$, and $\widetilde{\varphi}:=\varphi \uparrow \widetilde{T}^{2}$. Then

$\left({ }^{*}\right) \quad \mathcal{U} \ell\left(\mathbb{V} ; \widetilde{\mathcal{U}}^{2}\right) \vDash “ \varphi / \widetilde{T}^{2}: \mathcal{U}^{2} / \widetilde{T}^{2} \rightarrow \widetilde{\varphi}^{*}\left(\mathcal{U}^{1} / \widetilde{T}^{1}\right) "$ and

$(* *) \quad \mathcal{U} \ell\left(\mathbb{V} ; \widetilde{\mathcal{U}}^{2}\right) \vDash “\left(\varphi / \widetilde{T}^{2}\right)^{*}: \mathcal{U} \ell t\left(\mathbb{V} ; \mathcal{U}^{2} / \widetilde{T}^{2}\right) \rightarrow \mathcal{U} \ell t\left(\mathbb{V} ; \widetilde{\varphi}^{*}\left(\mathcal{U}^{1} / \widetilde{T}^{1}\right) ”\right.$.

For all $f \in \mathbb{V}^{\Sigma \mathcal{U}^{1}}$ also

$(* * *) \quad \mathcal{U} \ell\left(\mathbb{V} ; \widetilde{\mathcal{U}}^{2}\right) \vDash “ \Omega_{\widetilde{T}^{2}, \mathcal{U}^{2}}\left(\varphi^{*}(f)\right)={ }_{\mathcal{U}^{2} / \widetilde{T}^{2}}\left(\varphi / \widetilde{T}^{2}\right)^{*}\left(\widetilde{\varphi}^{*}\left(\Omega_{\widetilde{T}^{1}, \mathcal{U}^{1}}(f)\right)\right) "$,

ie, the following diagram commutes.

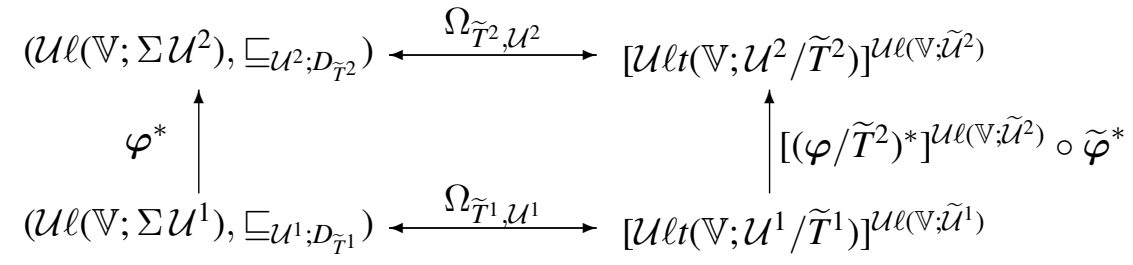

The $*$ in $\left(\varphi / \widetilde{T}^{2}\right)^{*}$ is here to be evaluated in $\mathcal{U} \ell\left(\mathbb{V} ; \widetilde{\mathcal{U}}^{2}\right)$ !

Proof From Definition 6.3 we get immediately that $\widetilde{\varphi}: \widetilde{\mathcal{U}}^{2} \rightarrow \widetilde{\mathcal{U}}^{1}$. For $t \in \Sigma \widetilde{T}^{2} \cap$ $\operatorname{dom} \varphi \in \Sigma \widetilde{\mathcal{U}}^{2}$ we have $\widetilde{\varphi}^{*}\left(\mathcal{U}^{1} / \widetilde{T}^{1}\right)(t)=\left(\mathcal{U}^{1} / \widetilde{T}^{1}\right)(\varphi(t))=\mathcal{U}_{\varphi(t)}^{1}$, and $\varphi_{t}: \mathcal{U}_{t}^{2} \rightarrow \mathcal{U}_{\varphi(t)}^{1}$ is a morphism. This is precisely the meaning of $(*)$.

(**) follows from $(*)$ by Proposition 7.6, which is satisfied in $\mathcal{U} \ell\left(\mathbb{V} ; \widetilde{\mathcal{U}}^{2}\right)$.

Finally, for $t \in \Sigma \widetilde{T}^{2} \cap \operatorname{dom} \varphi \in \Sigma \widetilde{\mathcal{U}}^{2}$ and $s \in \Sigma T_{t}^{2} \in \Sigma \mathcal{U}_{t}^{2}, \quad \Omega_{\widetilde{T}^{2}, \mathcal{U}^{2}}\left(\varphi^{*}(f)\right)(t)(s)=$ $\varphi^{*}(f)_{t}(s)=\varphi^{*}(f)(t \frown s)=f(\varphi(t \frown s))=f\left(\varphi(t) \frown \varphi_{t}(s)\right)=f_{\varphi(t)}\left(\varphi_{t}(s)\right)$ and $\left[\left(\varphi / \widetilde{T}^{2}(t)\right)^{*}\left(\widetilde{\varphi}^{*}\left(\Omega_{\widetilde{T}^{1}, \mathcal{U}^{1}}(f)\right)\right)(t)\right](s)=\varphi_{t}^{*}\left(\widetilde{\varphi}^{*}\left(f / \widetilde{T}^{1}\right)(t)\right)(s)=\varphi_{t}^{*}\left(\left(f / \widetilde{T}^{1}\right)(\varphi(t))(s)=\right.$ $\varphi_{t}^{*}\left(f_{\varphi(t)}\right)(s)=f_{\varphi(t)}\left(\varphi_{t}(s)\right)$. This proves $(* * *)$. 


\section{Elementary Relative Set Theory.}

Our goal in this section is to axiomatize stratified ultrapowers of the universe, in imitation of Section 3. Stratified ultrapowers are interpretations for the $\{\in, \sqsubseteq\}-$ language; therefore the desired theory SST is formulated in this language.

We define $\mathbb{S}_{\alpha}:=\{x: x \sqsubseteq \alpha\}$, the class of sets at level $\alpha$, or $\alpha$-standard sets; $\mathbb{V}:=\{x: x=x\}$ is the class of all sets. In particular, $\mathbb{S}_{0}=\{x: x \sqsubseteq 0\}$ is the class of all standard sets, and the unary predicate st is defined by: st $(x)$ iff $x \sqsubseteq 0$.

The axioms of SST are:

$\sqsubseteq$ is a total preordering of $\mathbb{V}$ with a least element 0 ;

$(\forall \alpha)\left[\left(\mathbb{V},=, \in, \mathbb{S}_{\alpha}\right) \vDash \mathbf{S T}\right]$

Explicitly:

\section{Relativization}

$(\forall x)(x \sqsubseteq x) ;(\forall x, y, z)(x \sqsubseteq y \wedge y \sqsubseteq z \Rightarrow x \sqsubseteq z) ;(\forall x, y)(x \sqsubseteq y \vee y \sqsubseteq x) ;(\forall x)(0 \sqsubseteq x)$.

ZFC for $\mathbb{S}_{\alpha}:(\forall \alpha) \mathcal{P}^{\mathbb{S}_{\alpha}}$, where $\mathcal{P}$ is any axiom of $\mathbf{Z F C}$.

Transfer from/into $\mathbb{S}_{\alpha}:(\forall \alpha)\left(\forall x_{1}, \ldots, x_{k} \in \mathbb{S}_{\alpha}\right)\left(\mathcal{P}^{\mathbb{S}_{\alpha}}\left(x_{1}, \ldots, x_{k}\right) \Leftrightarrow \mathcal{P}\left(x_{1}, \ldots, x_{k}\right)\right)$, where $\mathcal{P}\left(x_{1}, \ldots, x_{k}\right)$ is any $\in$-formula.

Inner Standardization into $\mathbb{S}_{\alpha}:(\forall \alpha)(\forall x)\left(\exists a \in \mathbb{S}_{\alpha}\right)\left(\forall z \in \mathbb{S}_{\alpha}\right)(z \in a \Leftrightarrow z \in x)$.

Remarks We use Greek letters $\alpha, \beta, \gamma$ as variables over sets when we are interested only in their level of standardness.

In terms of the levels $\mathbb{S}_{\alpha}$, the Relativization Axiom states that $(\forall \alpha)\left(\alpha \in \mathbb{S}_{\alpha}\right)$, $(\forall \alpha, \beta)\left(\alpha \in \mathbb{S}_{\beta} \Rightarrow \mathbb{S}_{\alpha} \subseteq \mathbb{S}_{\beta}\right),(\forall \alpha, \beta)\left(\mathbb{S}_{\alpha} \subseteq \mathbb{S}_{\beta} \vee \mathbb{S}_{\beta} \subseteq \mathbb{S}_{\alpha}\right)$, and $(\forall \alpha)\left(\mathbb{S}_{0} \subseteq \mathbb{S}_{\alpha}\right)$

It suffices to postulate $\mathbf{Z F C}$ for $\mathbb{S}_{0}$. Indeed, an axiom $\mathcal{P}$ of $\mathbf{Z F C}$ has no free variables, hence $\mathcal{P}^{\mathbb{S}_{0}}$ implies $\mathcal{P}$, by Transfer from $\mathbb{S}_{0}$, and $\mathcal{P}$ implies $\mathcal{P}^{\mathbb{S}_{\alpha}}$, by Transfer into $\mathbb{S}_{\alpha}$. In particular, SST implies ZFC.

The set $a$ in Inner Standardization is uniquely determined by $x$ and $\alpha$; we denote it $\mathbf{s h}_{\alpha}(x)$ and call it the $\alpha$-shadow of $x$.

Proposition 8.1 $\mathcal{U} \ell t(\mathbb{V} ; \mathcal{U}) \vDash \mathbf{S S T}$, for any TOU $\mathcal{U}$.

Moreover, if $\mathcal{U}(t)$ is $\kappa$-good for all $t \in \operatorname{dom} \mathcal{U}$ and $\mathcal{U} \ell t(\mathbb{V} ; \mathcal{U}) \vDash$ " $\alpha \sqsubset \beta$ for some $\beta$ ", then $\mathcal{U} \ell t(\mathbb{V} ; \mathcal{U}) \vDash(\kappa \text {-Idealization })^{\mathbb{S}_{\alpha}}$. 
Proof The fact that $\sqsubseteq \mathcal{U}$ has the properties required by Relativization is pointed out following its definition 7.1. Let $T^{\prime}:=T(f)$; by Corollary 7.3 then $g \sqsubseteq_{\mathcal{U}} f \Leftrightarrow$ $T(g) \preccurlyeq \mathcal{U} T(f) \Leftrightarrow g \in \boldsymbol{\pi}_{T^{\prime}, T}^{*}\left[\mathbb{V}^{\Sigma \mathcal{U}^{\prime}}\right]$. By Factoring Lemma, $\left(\mathcal{U} \ell(\mathbb{V} ; \mathcal{U}), \pi_{T^{\prime}, T}^{*}\left[\mathbb{V}^{\Sigma \mathcal{U}^{\prime}}\right]\right)$ is isomorphic to $\left[\mathcal{U} \ell t\left(\mathbb{V} ; \Sigma\left(\mathcal{U} / T^{\prime}\right)\right)\right]^{\mathcal{U} \ell\left(\mathbb{V} ; \Sigma \mathcal{U}^{\prime}\right)} \vDash \mathbf{S T}$ (Corollary 3.9 and Proposition 1.4). So $\mathbf{S T}$ relativizes to every level of $\mathcal{U} \ell t(\mathbb{V} ; \mathcal{U})$.

Let $T^{\prime}:=T(f) \prec \mathcal{U} T$; under the assumptions of $\kappa$-goodness, $\mathcal{U} \ell\left(\mathbb{V} ; \Sigma \mathcal{U}^{\prime}\right) \vDash$ " $\Sigma\left(\mathcal{U} / T^{\prime}\right)$ is $\mathfrak{k}_{\Sigma \mathcal{U}^{\prime}}(\kappa)$-good", hence $\mathcal{U} \ell\left(\mathbb{V} ; \Sigma \mathcal{U}^{\prime}\right) \vDash$ “U $\ell t\left(\mathbb{V} ; \mathcal{U} / T^{\prime}\right) \vDash \kappa$-Idealization”, and therefore $\mathcal{U} \ell t(\mathbb{V} ; \mathcal{U}) \vDash(\kappa \text {-Idealization })^{\mathbb{S}_{f}}$.

\section{More notation:}

We write $x \sqsubset y$ for $x \sqsubseteq y \wedge \neg y \sqsubseteq x$, and $x \boxminus y$ for $x \sqsubseteq y \wedge y \sqsubseteq x$.

$x \sqsubseteq \alpha y$ iff $(x \sqsubseteq \alpha \wedge y \sqsubseteq \alpha) \vee x \sqsubseteq y$ iff $x \sqsubseteq \alpha \vee x \sqsubseteq y$.

$x \sqsubset_{\alpha} y$ and $x \boxminus_{\alpha} y$ have the expected meaning.

We note that $x \sqsubseteq_{\alpha} 0 \Leftrightarrow x \sqsubseteq \alpha \Leftrightarrow x \in \mathbb{S}_{\alpha}$, so $\sqsubseteq_{\alpha}$ is a "relative" version of $\sqsubseteq$, where $\mathbb{S}_{\alpha}$ rather than $\mathbb{S}_{0}$ is taken as the coarsest level of standardness, and the finer levels remain unchanged.

If $\mathcal{P}$ is any $\in$ - $\sqsubseteq$-formula, $\mathcal{P}^{\alpha}$ denotes the formula obtained from $\mathcal{P}$ by replacing each occurrence of $\sqsubseteq$ by $\sqsubseteq_{\alpha}$.

\section{Proposition 8.2 (SST)}

If $\beta \sqsubseteq \alpha$, then $\left(x \sqsubseteq_{\beta} y\right)^{\alpha} \Leftrightarrow x \sqsubseteq_{\alpha} y$. If $\alpha \sqsubseteq \beta$, then $\left(x \sqsubseteq_{\beta} y\right)^{\alpha} \Leftrightarrow x \sqsubseteq_{\beta} y$.

If $\mathcal{P}$ is an $\in$-formula, then $\left(\mathcal{P}^{\mathbb{S}_{\alpha}}\right)^{\beta} \Leftrightarrow \mathcal{P}^{\mathbb{S}_{\alpha}}$ if $\beta \sqsubseteq \alpha$ and $\left(\mathcal{P}^{\mathbb{S}_{\alpha}}\right)^{\beta} \Leftrightarrow \mathcal{P}^{\mathbb{S}_{\beta}}$ if $\alpha \sqsubseteq \beta$.

In particular, $\left(\forall x_{1}, \ldots, x_{k}\right)\left(\mathcal{P}^{0}\left(x_{1}, \ldots, x_{k}\right) \Leftrightarrow \mathcal{P}\left(x_{1}, \ldots, x_{k}\right)\right)$.

Proof $(x \sqsubseteq \beta y)^{\alpha} \Leftrightarrow(x \sqsubseteq \beta \vee x \sqsubseteq y)^{\alpha} \Leftrightarrow\left(x \sqsubseteq_{\alpha} \beta \vee x \sqsubseteq_{\alpha} y\right) \Leftrightarrow(x \sqsubseteq \alpha \vee x \sqsubseteq \beta$ $\vee x \sqsubseteq \alpha \vee x \sqsubseteq y)$. This is equivalent to $x \sqsubseteq_{\alpha} y$ if $\beta \sqsubseteq \alpha$, and to $x \sqsubseteq_{\beta} y$ if $\alpha \sqsubseteq \beta$.

Definition 8.3 A set $L$ is a level set iff $L$ is finite, $\sqsubseteq\lceil L$ is a set and $(\forall x, y \in L)$ $(x \boxminus y \Rightarrow x=y)$. It follows that $\sqsubseteq\lceil L$ is a well-ordering.

For each level set $L \neq 0$ there is a unique sequence $\left\langle x_{n}: n \leq \nu\right\rangle(\nu \in \omega)$ such that $L=\left\{x_{n}: n \leq \nu\right\}$ and for $m, n \leq \nu, m<n \Leftrightarrow x_{m} \sqsubset x_{n}$; we call $\left\langle x_{n}: n \leq \nu\right\rangle$ a sequence of levels. In Section 10 we study in great detail particular sequences of levels, called there pedigrees.

Strong Support Principle: Let $\mathcal{P}(\bar{x})$ be any $\epsilon-\sqsubseteq$-formula.

For every $\bar{x}$ there is a level set $L$ such that $(\forall \alpha)\left[\mathcal{P}^{\alpha}(\bar{x}) \Leftrightarrow(\exists \beta \in L)(\alpha \boxminus \beta)\right]$. 
Theorem 8.4 $\mathcal{U} \ell t(\mathbb{V} ; \mathcal{U})$ satisfies the Strong Support Principle.

Proof Without loss of generality we assume that $\mathcal{P}$ has one free variable. It suffices to prove:

For every $x$ there is a level set $M$ such that

$$
(\forall \alpha)\left[\mathcal{P}^{\alpha}(x) \wedge \alpha \sqsupset 0 \Leftrightarrow(\exists \beta \in M)(\alpha \boxminus \beta)\right] .
$$

We then let $L:=M \cup\{0\}$ if $\mathcal{P}^{0}(x)(\Leftrightarrow \mathcal{P}(x))$ holds; $L:=M$ otherwise.

We proceed by induction on $\operatorname{rank} T$.

(0) If $T=T^{(0)}$, then $\mathcal{U} \ell t(\mathbb{V} ; \mathcal{U}) \vDash(\forall \alpha)(\alpha \boxminus 0)$ and we let $M=\mathfrak{k}_{\Sigma \mathcal{U}}(0)$.

(1) Let $T=\{0\} \cup \bigcup_{i \in I}\langle i\rangle \frown T_{i}, \quad x=f \in \mathbb{V}^{\Sigma \mathcal{U}}$. We recall that $T^{(1)}=\{0\} \cup\{\langle i\rangle$ : $i \in I\} \preccurlyeq_{U} T, T^{(0)} \prec_{\mathcal{U}} T^{(1)}$, and $T^{(0)} \prec_{\mathcal{U}} T^{\prime} \Rightarrow T^{(1)} \preccurlyeq_{\mathcal{U}} T^{\prime}$ for every $T^{\prime} \preccurlyeq_{\mathcal{U}} T$. Let $\gamma:=D_{T^{(1)}}$. In terms of $\sqsubseteq$, these observations amount to: $\mathcal{U} \ell t(\mathbb{V} ; \mathcal{U}) \vDash 0 \sqsubset \alpha$ iff $\mathcal{U} \ell t(\mathbb{V} ; \mathcal{U}) \vDash \gamma \sqsubseteq \alpha$. Let $\widetilde{I}:=\left\{i \in I: \operatorname{dom} f_{i} \in \mathcal{U}_{i}\right\} ; \widetilde{I} \in \mathcal{U}(0)$. By the inductive assumption, for each $i \in \widetilde{I}$ there is $M_{i} \in \mathbb{V}^{\Sigma \mathcal{U}_{i}}$ such that $\left(\mathcal{U} \ell\left(\mathbb{V} ; \Sigma \mathcal{U}_{i}\right)\right.$, $\left.\mathcal{U}_{i}\right) \vDash$ " $M_{i}$ is a level set $\wedge(\forall \alpha)\left[\mathcal{P}^{\alpha}\left(f_{i}\right) \wedge \alpha \sqsupset 0 \Leftrightarrow\left(\exists \beta \in M_{i}\right)(\alpha \boxminus \beta)\right]$ ".

Let $\mathcal{U}^{(1)}:=\mathcal{U} \uparrow T^{(1)}$; we recall that $\Sigma \mathcal{U}^{(1)}$ is isomorphic to $\mathcal{U}(0)$ via the mapping $\langle i\rangle \mapsto i$. Let $\widetilde{M} \in \mathbb{V}^{\Sigma \mathcal{U}}$ be defined by $\widetilde{M}(\langle i\rangle \frown s)=M_{i}(s)$ for $i \in \widetilde{I}, s \in \Sigma T_{i}$, so that $\Omega(\widetilde{M})=\widetilde{M} / T^{(1)}=_{\Sigma \mathcal{U}^{(1)}}\left\langle M_{i}: i \in \widetilde{I}\right\rangle$. By the Factoring Theorem 7.11 (with $\left.T^{\prime}=T^{(1)}\right),(\mathcal{U} \ell(\mathbb{V} ; \Sigma \mathcal{U}), \sqsubseteq \mathcal{U} ; \gamma) \vDash “ \widetilde{M}$ is a level set $\wedge(\forall \alpha)\left[\mathcal{P}^{\alpha}(f) \wedge \alpha \sqsupset 0 \Leftrightarrow(\exists \beta \in\right.$ $\widetilde{M})(\alpha \boxminus \beta)]$ ". We have $\alpha \sqsubseteq_{\mathcal{U} ; \gamma} \beta \Leftrightarrow \alpha \sqsubseteq_{\mathcal{U}} \beta$ for $\gamma \sqsubset_{\mathcal{U}} \alpha, \beta$, and $\left[\left(\mathcal{U} \ell(\mathbb{V} ; \Sigma \mathcal{U})\right.\right.$, $\sqsubseteq_{\mathcal{U} ; \gamma}$ )$\left.\vDash \mathcal{P}^{\alpha}(f)\right] \Leftrightarrow\left[\mathcal{U} \ell t(\mathbb{V} ; \mathcal{U})=(\mathcal{U} \ell(\mathbb{V} ; \Sigma \mathcal{U}), \sqsubseteq \mathcal{U}) \vDash \mathcal{P}^{\alpha}(f)\right]$ for $\gamma \sqsubset \mathcal{U} \alpha$. Hence $\mathcal{U} \ell t(\mathbb{V} ; \mathcal{U}) \vDash " \widetilde{M}$ is a level set $\wedge(\forall \alpha)\left[\mathcal{P}^{\alpha}(f) \wedge \alpha \sqsupset \gamma \Leftrightarrow(\exists \beta \in \widetilde{M})(\alpha \boxminus \beta)\right]$ ". If also $\mathcal{U} \ell t(\mathbb{V} ; \mathcal{U}) \vDash \mathcal{P}^{\gamma}(f)$, we take $M \in \mathbb{V}^{\Sigma \mathcal{U}}$ such that $\mathcal{U} \ell t(\mathbb{V} ; \mathcal{U}) \vDash M=\widetilde{M} \cup\{\gamma\}$; otherwise, we let $M=\widetilde{M}$. It is easy to verify that $\left({ }^{*}\right)$ holds in $\mathcal{U} \ell t(\mathbb{V} ; \mathcal{U})$ for $x=f$ and this $M$.

SST $^{*}$ is the theory obtained by adding the following axioms to SST:

Block Standardization: $(\forall x \sqsupset 0)(\exists a \sqsubset x)(\forall z \sqsubset x)(z \in a \Leftrightarrow z \in x)$.

Granularity: Let $\mathcal{P}(\bar{x})$ be any $\in$ - $\sqsubseteq$-formula.

For all $x_{1}, \ldots, x_{k}$, if $(\exists \alpha) \mathcal{P}^{\alpha}\left(x_{1}, \ldots, x_{k}\right)$, then

$$
(\exists \alpha)\left[\mathcal{P}^{\alpha}\left(x_{1}, \ldots, x_{k}\right) \wedge(\forall \beta)\left(\beta \sqsubset \alpha \Rightarrow \neg \mathcal{P}^{\beta}\left(x_{1}, \ldots, x_{k}\right)\right)\right] .
$$


Remarks Block Standardization is equivalent to

$$
(\forall \alpha \sqsupset 0)(\forall x)(\exists a \sqsubset \alpha)(\forall z \sqsubset \alpha)(z \in a \Leftrightarrow z \in x) .
$$

To prove the new version, apply the original version to $\operatorname{sh}_{\alpha}(x)$ in place of $x$ to obtain $a$ if $\mathbf{s h}_{\alpha}(x) \boxminus \alpha$; if $\mathbf{s h}_{\alpha}(x) \sqsubset \alpha$, let $a=\mathbf{s h}_{\alpha}(x)$. For the converse, let $\alpha=x$.

Corollary $8.5 \mathcal{U} \ell t(\mathbb{V} ; \mathcal{U})$ satisfies SST*, for any TOU $\mathcal{U}$.

Proof If $L$ is a level set for $\mathcal{P}$ as in the Strong Support Principle and $(\exists \alpha) \mathcal{P}^{\alpha}(\bar{x})$ holds, then $L \neq 0$. To prove Granularity, we take $\alpha$ to be the $\sqsubseteq$-least element of $L$.

Another consequence of the Strong Support Principle is the existence of a level set $L$ such that for every $\alpha$ there is $\beta \in L$ with $\alpha \boxminus \beta$ [take $\mathcal{P}(x)$ to be $x=x$ and fix any $x$ ]. As $L$ is finite and well-ordered by $\sqsubseteq$, every level $\alpha$ except the least one has an immediate predecessor level; we denote it $\alpha^{-}$. Block Standardization follows from Inner Standardization into $\mathbb{S}_{\alpha^{-}}$.

Proposition 8.6 (SST $\left.{ }^{*}\right)$ If $(\forall x, y \in M)(x \boxminus y \Rightarrow x=y)$, then $M$ is a level set.

Proof Claim 1. $M \subseteq \mathbb{S}_{\alpha_{0}}$, where $\alpha_{0} \boxminus M$.

Proof of Claim 1. Suppose some $m \in M \backslash \mathbb{S}_{\alpha_{0}}$ There is a one-one mapping $f \in \mathbb{S}_{\alpha_{0}}$ of $\mu$ onto $M$, where $\mu$ is a (finite or infinite) cardinal. If $\mu=1, M=\{m\}$ and $m \in \mathbb{S}_{\alpha_{0}}$ by Transfer. So $\mu>1$ and $m=f(\xi)$ for $\xi<\mu$. Either $\eta:=\xi+1<\mu$ or $\eta:=\xi-1<\mu$ [the second case is needed if $\mu=\xi+1$ ]. In either case, $\eta \neq \xi$ and $\eta \boxminus \xi$. As $f, f^{-1} \in \mathbb{S}_{\alpha_{0}}$, it follows that $m^{\prime}:=f(\eta) \sqsubseteq \alpha_{0} m$ and $m \sqsubseteq \alpha_{0} m^{\prime}$, so $m \boxminus m^{\prime}$ while $m \neq m^{\prime}$, a contradiction.

Let $\mathcal{P}^{\alpha}(M)$ be the statement

"There exists $L \subseteq M$ such that $L$ is finite, $(\forall x, y \in L)\left(x \boxminus_{\alpha} y \Rightarrow x=y\right)$, $\sqsubseteq_{\alpha}\lceil L$ is a set, $\left(\forall x \notin \mathbb{S}_{\alpha}\right)(x \in M \Leftrightarrow x \in L)$, and $(\forall x \in L)\left(x \notin \mathbb{S}_{\alpha}\right)$ ".

It is immediate from Claim 1 that $\mathcal{P}^{\alpha_{0}}(M)$ holds: let $L=0$. Let $L^{\prime}$ be a witness to the validity of $\mathcal{P}^{\alpha}(M)$ where $0 \sqsubset \alpha$. If there is $x \in M$ such that $x \boxminus \alpha$, let $L:=L^{\prime} \cup\{x\}$; otherwise let $L:=L^{\prime}$. By Block Standardization, there is a set $B \sqsubset \alpha$ such that $(\forall z \sqsubset \alpha)(z \in B \Leftrightarrow z \in M \backslash L)$; then $M \backslash L \subseteq B$.

Claim 2. $M \backslash L \subseteq \mathbb{S}_{\beta}$, where $\beta \boxminus B$.

Proof of Claim 2. Suppose some $m \in M \backslash L, m \sqsupset \beta$. Let $f \in \mathbb{S}_{\beta}$ be a one-one mapping of a cardinal $\mu$ onto $B$. Since $M \backslash L \subseteq B$, we have $m=f(\xi)$ for some 
$\xi<\mu$. As in the proof of Claim 1, we find $\eta \neq \xi$ such that $m^{\prime}:=f(\eta) \boxminus m \sqsubset \alpha$. We have $m^{\prime} \in B$, so $m^{\prime} \in M \backslash L$; but $m^{\prime} \neq m$, a contradiction.

The set $L$ witnesses the validity of $\mathcal{P}^{\beta}(M)$ where $\beta \sqsubset \alpha$. By Granularity, $\mathcal{P}^{0}(M)$ holds. If $L^{\prime}$ is a witness to $\mathcal{P}^{0}(M)$, and there is $x \in M$ such that $x \boxminus 0$, let $L:=L^{\prime} \cup\{x\}$; otherwise let $L:=L^{\prime}$. It is clear that $L$ is a level set and $M=L$.

\section{Stratified Ultrafilters.}

We recall that $\boldsymbol{\beta} X$ is the set of all ultrafilters over $X$-the Stone-Čech space over $X$, and $U \sim V$ means that $U \cap V$ is an ultrafilter. For an arbitrary nonempty set $A$ we define by recursion on ordinals:

(0) $\boldsymbol{\beta}_{0} A:=A$

(1) For $\xi>0, \boldsymbol{\beta}_{<\xi} A:=\bigcup_{\eta<\xi} \boldsymbol{\beta}_{\eta} A$ and $\boldsymbol{\beta}_{\xi} A:=\boldsymbol{\beta}_{<\xi} A \cup\left\{U \in \boldsymbol{\beta}\left(\boldsymbol{\beta}_{<\xi} A\right): U\right.$ is nonprincipal and $\boldsymbol{\beta}_{<\eta} A \notin U$ for any $\left.\eta<\xi\right\}=\boldsymbol{\beta}_{<\xi} A \cup\left\{U \in \boldsymbol{\beta}\left(\boldsymbol{\beta}_{<\xi} A\right): U\right.$ is nonprincipal and there is no $V \in \boldsymbol{\beta}_{<\xi} A$ such that $\left.U \sim V\right\}$.

Elements of $\boldsymbol{\beta}_{\infty} A:=\bigcup_{\xi \in \mathbb{O} n} \boldsymbol{\beta}_{\xi} A$ are called stratified ultrafilters over $A$. For $U \in \boldsymbol{\beta}_{\infty} A$ we let $\operatorname{Dom} U:=A$. As usual, the recursive definition assigns to each stratified ultrafilter an ordinal rank.

We are taking a terminological liberty by calling elements of $A$ stratified ultrafilters. There is a natural identification of each $a \in \boldsymbol{\beta}_{0} A$ with the principal ultrafilter $W_{a, A}$ it generates over $A$. We choose not to make this identification formally, as it would lead to technical complications elsewhere.

Stratified ultrafilters of rank 1 are the nonprincipal ultrafilters over $A$; stratified ultrafilters of rank 2 are the nonprincipal ultrafilters over $\boldsymbol{\beta} A$ that concentrate on nonprincipal ultrafilters over $A$ [ie, such that $(\boldsymbol{\beta} A \backslash A) \in U$ ], and so on.

Proposition 9.1 For $U, V \in \boldsymbol{\beta}_{\infty} A \backslash A, U \sim V$ implies $U=V$.

Proof Assume without loss of generality that $\operatorname{rank} V \leq \operatorname{rank} U=\xi$. If $\operatorname{rank} U=$ rank $V$, then $U$ and $V$ are ultrafilters over the same domain $\boldsymbol{\beta}_{<\xi} A$, and $U \sim V$ implies $U=V$. If rank $V<\operatorname{rank} U$, then $U \sim V$ is impossible by the definition of $\boldsymbol{\beta}_{\xi} A$.

Proposition 9.2 For every ultrafilter $W$ with $\operatorname{dom} W \subseteq \boldsymbol{\beta}_{\infty} A$ there is a unique $V \in \boldsymbol{\beta}_{\infty} A$ such that either $W \sim V \notin \boldsymbol{\beta}_{0} A$ or $W$ is principal and $V$ is its generator. 
Proof If $W$ is nonprincipal, fix $\xi$ such that $\operatorname{dom} W \subseteq \boldsymbol{\beta}_{<\xi} A$ and extend $W$ to $\bar{W} \sim W$ with $\operatorname{dom} \bar{W}=\boldsymbol{\beta}_{<\xi} A$. Then either $\bar{W} \in \boldsymbol{\beta}_{\xi} A$, and we let $V:=\bar{W}$, or there is $V \in \boldsymbol{\beta}_{<\xi} A \backslash \boldsymbol{\beta}_{0} A$ such that $\bar{W} \sim V$. Uniqueness of $V$ follows from the preceding proposition.

We let $\mathfrak{m}(W):=V$. Note that $W_{1} \sim W_{2}$ implies $\mathfrak{m}\left(W_{1}\right)=\mathfrak{m}\left(W_{2}\right)$.

Definition 9.3 Let $f: A \rightarrow B$. We define its extension $\bar{f}: \boldsymbol{\beta}_{\infty} A \rightarrow \boldsymbol{\beta}_{\infty} B$ by recursion.

(0) For $a \in \boldsymbol{\beta}_{0} A$ we let $\bar{f}_{0}(a)=f(a) \in \boldsymbol{\beta}_{0} B$.

(1) Assume $\xi>0$ and $\bar{f}_{<\xi}:=\bigcup_{\eta<\xi} \bar{f}_{\eta}: \boldsymbol{\beta}_{<\xi} A \rightarrow \boldsymbol{\beta}_{<\xi} B$ is already defined. We let $\bar{f}_{\xi}(U)=\bar{f}_{<\xi}(U)$ for $U \in \boldsymbol{\beta}_{<\xi} A$. For $U \in \boldsymbol{\beta}_{\xi} A \backslash \boldsymbol{\beta}_{<\xi} A$ we let $W:=\bar{f}_{<\xi}[U] \in$ $\boldsymbol{\beta}\left(\boldsymbol{\beta}_{<\xi} B\right)$ and $\bar{f}_{\xi}(U):=\mathfrak{m}(W) \in \boldsymbol{\beta}_{\xi} B$. Finally $\bar{f}:=\bigcup_{\xi \in \mathbb{O} n} \bar{f}_{\xi}$.

We often write $\bar{f}[U]$ in place of $\bar{f}_{<\xi}[U]$. Note that the rank of $\bar{f}(U)$ is less than or equal to the rank of $U$, and $\bar{f}(U)=\bar{f}[U]$ if and only if the equality holds.

Proposition 9.4 If $f: A \rightarrow B$ and $g: B \rightarrow C$, then $\overline{g \circ f}=\bar{g} \circ \bar{f}$. Trivially also $\overline{I d_{A}}=\mathbb{I} d_{\boldsymbol{\beta}_{\infty} A}$.

Proof By induction on rank of $U \in \boldsymbol{\beta}_{\infty} A$. The claim is trivial if $U \in \boldsymbol{\beta}_{0} A$. Let $U \in \boldsymbol{\beta}_{\xi} A \backslash \boldsymbol{\beta}_{<\xi} A$ and $W=\bar{f}_{<\xi}[U]$; then $\bar{f}_{\xi}(U)=\mathfrak{m}(W)$.

If $W$ is principal, say generated by $V \in \boldsymbol{\beta}_{<\xi} B$, then $\bar{g}_{<\xi}[W]$ is generated by $\bar{g}_{\xi}(V)=$ $\bar{g}_{<\xi}(V) \in \boldsymbol{\beta}_{<\xi} C$ and $\overline{g \circ f}(U)=\overline{g \circ f}_{\xi}(U)=\mathfrak{m}\left(\overline{g \circ f}_{<\xi}[U]\right)=\mathfrak{m}\left(\bar{g}_{<\xi}[W]\right)=$ $\bar{g}_{\xi}(V)=\bar{g}_{\xi}(\mathfrak{m}(W))=\bar{g}_{\xi}\left(\bar{f}_{\xi}(U)\right)=\bar{g}(\bar{f}(U))$.

If $W$ is nonprincipal, we have $\bar{f}_{<\xi}[U]=W \sim \mathfrak{m}(W)=\bar{f}_{\xi}(U)$. Hence $\bar{g}_{<\xi}[W] \sim$ $\bar{g}_{<\xi}\left[\bar{f}_{\xi}(U)\right]=\bar{g}_{<\eta}\left[\bar{f}_{\xi}(U)\right]$ where $\eta \leq \xi$ is the rank of $\bar{f}_{\xi}(U)$. We get $\overline{g \circ f}(U)=$ $\bar{g}^{\circ \circ f_{\xi}}(U)=\mathfrak{m}\left(\bar{g}^{\circ \circ f} f_{<\xi}[U]\right)=\mathfrak{m}\left(\bar{g}_{<\xi}[W]\right)=\mathfrak{m}\left(\bar{g}_{<\eta}\left[\bar{f}_{\xi}(U)\right]\right)=\bar{g}_{\eta}\left(\bar{f}_{\xi}(U)\right)=\bar{g}(\bar{f}(U))$. [The third equality is true by the inductive assumption.]

Definition 9.5 For $C \subseteq A$ we define $\boldsymbol{\beta}_{\xi} A / C \subseteq \boldsymbol{\beta}_{\xi} A$ (stratified ultrafilters concentrated on $C$ ) by recursion:

(0) $\boldsymbol{\beta}_{0} A / C:=C$

(1) For $\xi>0, \boldsymbol{\beta}_{<\xi} A / C:=\bigcup_{\eta<\xi} \boldsymbol{\beta}_{\eta} A / C$ and

$\boldsymbol{\beta}_{\xi} A / C:=\boldsymbol{\beta}_{<\xi} A / C \cup\left\{U \in \boldsymbol{\beta}_{\xi} A \backslash \boldsymbol{\beta}_{<\xi} A: \boldsymbol{\beta}_{<\xi} A / C \in U\right\}$.

Of course, $\boldsymbol{\beta}_{\infty} A / C:=\bigcup_{\xi \in \mathbb{O} n} \boldsymbol{\beta}_{\xi} A / C$. 
Remarks By induction on rank it is easily seen that $\boldsymbol{\beta}_{\infty} A / C_{1} \cap \boldsymbol{\beta}_{\infty} A / C_{2}=$ $\boldsymbol{\beta}_{\infty} A /\left(C_{1} \cap C_{2}\right)$. In particular, if $C_{1} \cap C_{2}=0$, then $\boldsymbol{\beta}_{\infty} A / C_{1} \cap \boldsymbol{\beta}_{\infty} A / C_{2}=0$.

Proposition 9.6 Let $A \cap B=C \neq 0$ and let $\rho: A \rightarrow B$ and $\sigma: B \rightarrow A$ be such that $\rho(x)=\sigma(x)=x$ for all $x \in C$. If $U \in \boldsymbol{\beta}_{\xi} A / C$ and $V \in \boldsymbol{\beta}_{\xi} B / C$, then $\bar{\rho}(U) \in \boldsymbol{\beta}_{\xi} B / C$, $\bar{\sigma}(V) \in \boldsymbol{\beta}_{\xi} A / C$, and $\bar{\sigma}(\bar{\rho}(U))=U, \bar{\rho}(\bar{\sigma}(V))=V$.

Remarks The assertion implies that $\bar{\rho}$ maps $\boldsymbol{\beta}_{\xi} A / C$ one-one onto $\boldsymbol{\beta}_{\xi} B / C$, and $\bar{\sigma}$ maps $\boldsymbol{\beta}_{\xi} B / C$ one-one onto $\boldsymbol{\beta}_{\xi} A / C$. Also, for $U \in \boldsymbol{\beta}_{\xi} A / C, \bar{\rho}(U)$ has the same rank as $U$, and so $\bar{\rho}(U)=\bar{\rho}[U]$. Similarly, for $V \in \boldsymbol{\beta}_{\xi} B / C, \bar{\sigma}(V)=\bar{\sigma}[V]$.

Proof (0) For $a \in C, \bar{\rho}(a)=\rho(a)=a$, and similarly $\bar{\sigma}(a)=a$.

(1) Assume that the assertion is true for all $\eta<\xi$. By the preceding remarks, $\bar{\rho}$ maps $\boldsymbol{\beta}_{<\xi} A / C$ one-one onto $\boldsymbol{\beta}_{<\xi} B / C$. If $U \in \boldsymbol{\beta}_{\xi} A / C \backslash \boldsymbol{\beta}_{<\xi} A / C$, then $\boldsymbol{\beta}_{<\xi} A / C \in$ $U, U$ is nonprincipal, and $\boldsymbol{\beta}_{<\eta} A / C=\boldsymbol{\beta}_{<\eta} A \cap \boldsymbol{\beta}_{<\xi} A / C \notin U$ for any $\eta<\xi$. Hence $\bar{\rho}\left[\boldsymbol{\beta}_{<\xi} A / C\right]=\boldsymbol{\beta}_{<\xi} B / C \in \bar{\rho}[U], \quad \bar{\rho}[U]$ is nonprincipal, and $\bar{\rho}\left[\boldsymbol{\beta}_{<\eta} A / C\right]=$ $\boldsymbol{\beta}_{<\eta} B / C \notin \bar{\rho}[U]$ for any $\eta<\xi$; hence $\boldsymbol{\beta}_{<\eta} B \notin \bar{\rho}[U]$ (note $\boldsymbol{\beta}_{<\eta} B / C=\boldsymbol{\beta}_{<\eta} B \cap$ $\left.\boldsymbol{\beta}_{<\xi} B / C\right)$. We conclude that $\bar{\rho}(U)=\bar{\rho}[U] \in \boldsymbol{\beta}_{\xi} B / C \backslash \boldsymbol{\beta}_{<\xi} B / C$.

By a symmetric argument, if $V \in \boldsymbol{\beta}_{\xi} B / C \backslash \boldsymbol{\beta}_{<\xi} B / C$, then $\bar{\sigma}(U)=\bar{\sigma}[U] \in \boldsymbol{\beta}_{\xi} A / C \backslash$ $\boldsymbol{\beta}_{<\xi} A / C$. For such $U, V$ we then have $\bar{\sigma}(\bar{\rho}(U))=\bar{\sigma}[\bar{\rho}[U]]=U$ because $\bar{\sigma} \circ \bar{\rho}$ is the identity on $\boldsymbol{\beta}_{<\xi} A / C \in U$, and $\bar{\rho}(\bar{\sigma}(V))=\bar{\rho}[\bar{\sigma}[V]]=V$ because $\bar{\rho} \circ \bar{\sigma}$ is the identity on $\boldsymbol{\beta}_{<\xi} B / C \in V$.

Proposition 9.7 For every $a \in A$ and $U \in \boldsymbol{\beta}_{\xi} B$ there is a unique $U_{a} \in \boldsymbol{\beta}_{\xi}(A \times B)$ such that $\overline{\pi_{1}}\left(U_{a}\right)=a$ and $\overline{\pi_{2}}\left(U_{a}\right)=U$.

Proof By induction on $\xi$.

If $\xi=0$, then $U=b$ for some $b \in B$, and $U_{a}:=\langle a, b\rangle$ has the required properties.

If $W \in \boldsymbol{\beta}_{0}(A \times B)$, then $W=\langle c, d\rangle$. From $\overline{\pi_{1}}(W)=a$ and $\overline{\pi_{2}}(W)=U=b$ it follows that $c=a, d=b$, so $W=U_{a}$.

Let now $\xi>0$ and $U \in \boldsymbol{\beta}_{\xi} B \backslash \boldsymbol{\beta}_{<\xi} B$.

Existence. By inductive assumption, there is the function $t: \boldsymbol{\beta}_{<\xi} B \rightarrow \boldsymbol{\beta}_{<\xi}(A \times B)$ defined by $t(V)=V_{a}$. We let $U_{a}:=t[U]$; ie, for $X \subseteq \boldsymbol{\beta}_{<\xi}(A \times B)$,

$$
X \in U_{a} \Leftrightarrow Y:=\left\{V \in \boldsymbol{\beta}_{<\xi} B: t(V)=V_{a} \in X\right\} \in U .
$$


Clearly $U_{a} \in \boldsymbol{\beta}\left(\boldsymbol{\beta}_{<\xi}(A \times B)\right)$. We note that $\overline{\pi_{2}}\left[U_{a}\right]=U$ : If $X \in U_{a}$, then $X \supseteq t[Y] \in$ $U_{a}$, and so $\overline{\pi_{2}}[X] \supseteq \bar{\pi}_{2}[t[Y]]=Y \in U$ [by inductive assumption, $\overline{\pi_{2}}\left(V_{a}\right)=V$ for all $V \in Y]$. So $U_{a}$ is nonprincipal, and not equivalent to an ultrafilter of smaller rank (otherwise, $U$ would be as well); hence $U_{a} \in \boldsymbol{\beta}_{\xi}(A \times B)$ and $\overline{\pi_{2}}\left(U_{a}\right)=\mathfrak{m}\left(\overline{\pi_{2}}\left[U_{a}\right]\right)=$ $\mathfrak{m}(U)=U$.

It remains to show that $\overline{\pi_{1}}\left(U_{a}\right):=\mathfrak{m}\left(\bar{\pi}_{1}\left[U_{a}\right]\right)=a$. But $X \in U_{a}$ implies $V_{a} \in X$ for some $V \in \operatorname{dom} U$, by (*); hence $a=\overline{\pi_{1}}\left(V_{a}\right) \in \overline{\pi_{1}}[X]$. We conclude that $\overline{\pi_{1}}\left[U_{a}\right]$ is a principal ultrafilter generated by $a$, hence $\overline{\pi_{1}}\left(U_{a}\right)=\mathfrak{m}\left(\overline{\pi_{1}}\left[U_{a}\right]\right)=a$.

Uniqueness. Let $W \in \boldsymbol{\beta}_{\xi}(A \times B) \backslash \boldsymbol{\beta}_{<\xi}(A \times B)$ and $\bar{\pi}_{1}(W)=a$; we prove that $W=U_{a}^{\prime}$ for some $U^{\prime} \in \boldsymbol{\beta}_{\xi} B$. Uniqueness follows: if also $\overline{\pi_{2}}(W)=U$, then $U=U^{\prime}\left(=\overline{\pi_{2}}\left(U_{a}^{\prime}\right)\right)$ and $W=U_{a}$.

The assumption $\overline{\pi_{1}}(W)=\mathfrak{m}\left(\overline{\pi_{1}}[W]\right)=a$ means that $\overline{\pi_{1}}[W]$ is principal generated by $a$; ie, $\{a\} \in \overline{\pi_{1}}[W]$; ie, there is $X \in W$ such that $(\forall V \in X)\left(\overline{\pi_{1}}(V)=a\right)$. Hence, by inductive assumption, for each $V \in X$ there is a unique $V^{\prime} \in \boldsymbol{\beta}_{\operatorname{rank}(V)} B$ such that $V=V_{a}^{\prime}$. We define $s$ on $X$ by $s(V)=V^{\prime}$ and let $U^{\prime}=s[W] \in \boldsymbol{\beta}\left(\boldsymbol{\beta}_{<\xi} B\right)$. We have to prove that $U^{\prime} \in \boldsymbol{\beta}_{\xi} B$ and $W=U_{a}^{\prime}$.

We note that $t(s(V))=t\left(V^{\prime}\right)=V_{a}^{\prime}=V[t$ is one-one and preserves rank because $\left.\overline{\pi_{2}}(t(V))=V\right]$. Hence $s$ is one-one and preserves rank. It follows that $U^{\prime} \in \boldsymbol{\beta}_{\xi} B$, and $U_{a}^{\prime}=t\left[U^{\prime}\right]=t[s[W]]=W$.

With each stratified ultrafilter $U$ we associate a TOU $\mathbf{U}$, and consequently also a stratified ultrapower $\mathcal{U} \ell t(\mathbb{V} ; \mathbf{U})$.

Definition 9.8 (0) If $U \in \boldsymbol{\beta}_{0} A$, then $\mathbf{U}=0$.

(1) If $U \in \boldsymbol{\beta}_{\xi} A \backslash \boldsymbol{\beta}_{<\xi} A$ for $\xi>0$ and, for each $V \in \operatorname{dom} U=\boldsymbol{\beta}_{\xi} A, \mathbf{V}$ is the TOU associated to $V$, we let $T_{\mathbf{U}}:=\{0\} \cup\left\{\langle V\rangle \frown T_{\mathbf{V}}: V \in \operatorname{dom} U\right\}, \mathbf{U}(0)=U$, and $\mathbf{U}(\langle V\rangle \frown t)=\mathbf{V}(t)$ for all $V \in \operatorname{dom} U$ and $t \in T_{\mathbf{V}} \backslash \Sigma T_{\mathbf{V}}$. Note in particular that $\mathbf{U}(\langle V\rangle)=V$, for all $V \in \operatorname{dom} U$.

It is easy to check that $\mathbf{U}$ is indeed a TOU according to Definition 6.1, and that the rank of $\mathbf{U}$ (as TOU) equals the rank of $U$ (as a stratified ultrafilter).

We also have the following explicit description of $\mathbf{U}$, easily verified by induction.

If $U \in \boldsymbol{\beta}_{0} A$, then $\mathbf{U}=0$; otherwise, let $L$ be the set of all finite sequences $\left\langle U_{1}, \ldots, U_{n}\right\rangle$ where $U_{1} \in \operatorname{dom} U, U_{\ell+1} \in \operatorname{dom} U_{\ell}$ for $1 \leq \ell<n$, and $U_{n} \in \boldsymbol{\beta}_{0} A$. Then $T_{\mathbf{U}}=L \downarrow$, $\Sigma T_{\mathbf{U}}=L, \mathbf{U}(0)=U$ and $\mathbf{U}\left(\left\langle U_{1}, \ldots, U_{\ell}\right\rangle\right)=U_{\ell}$ whenever $\left\langle U_{1}, \ldots, U_{\ell}\right\rangle \in T_{\mathbf{U}} \backslash \Sigma T_{\mathbf{U}}$ (ie, whenever $U_{\ell}$ is nonprincipal). 
Proposition 9.9 Every $f: A^{1} \rightarrow A^{2}$ induces a canonical morphism $\varphi: \mathbf{U}^{1} \rightarrow \mathbf{U}^{2}$ for any $U^{1} \in \boldsymbol{\beta}_{\infty} A^{1}$ and $U^{2}:=\bar{f}\left(U^{1}\right) \in \boldsymbol{\beta}_{\infty} A^{2}$. If $f=I d_{A^{1}}$, then $\boldsymbol{\varphi}=I d_{T_{\mathrm{U}^{1}}}$. If $g: A^{2} \rightarrow A^{3}$ induces $\psi: \mathbf{U}^{2} \rightarrow \mathbf{U}^{3}$ where $U^{3}:=\bar{g}\left(U^{2}\right)$, then $\psi \circ \varphi: \mathbf{U}^{1} \rightarrow \mathbf{U}^{3}$ is equal, everywhere on its domain, to the canonical morphism $\boldsymbol{\theta}$ induced by $g \circ f: A^{1} \rightarrow$ $A^{3}$.

Proof We describe $\varphi$ recursively. If $U^{1} \in \boldsymbol{\beta}_{0} A^{1}$, then $U^{2}=\bar{f}\left(U^{1}\right)=f\left(U^{1}\right) \in \boldsymbol{\beta}_{0} A^{2}$, $\mathbf{U}^{1}=\mathbf{U}^{2}=0$ and $\varphi$ is the trivial morphism $\varphi(0)=0$.

If $U^{1} \in \boldsymbol{\beta}_{\xi} A^{1} \backslash \boldsymbol{\beta}_{<\xi} A^{1}$, let $W:=\bar{f}\left[U^{1}\right]$.

Case (i): $W$ is principal, generated by $U^{2} \in \boldsymbol{\beta}_{<\xi} A^{2}$. Then $S:=\left\{V^{1} \in \boldsymbol{\beta}_{<\xi} A^{1}\right.$ : $\left.\bar{f}\left(V^{1}\right)=U^{2}\right\} \in U^{1}$. By the inductive assumption, for every $V^{1} \in S$ we have the canonical morphism $\varphi^{V^{1}}: \mathbf{V}^{1} \rightarrow \mathbf{U}^{2}$. We let $\varphi\left(\left\langle V^{1}\right\rangle \frown t\right):=\varphi^{V^{1}}(t)$ for all $t \in T_{\mathbf{V}^{1}}$, $V_{1} \in S$. Note in particular that $\varphi\left(\left\langle V^{1}\right\rangle\right)=0$ for all $V^{1} \in S$-this is the collapsing case in the definition of morphism.

Case (ii): $W$ is nonprincipal, $W \sim U^{2} \in \boldsymbol{\beta}_{\xi} A^{2}$. Then $S:=\left\{V^{1} \in \boldsymbol{\beta}_{<\xi} A^{1}:\right.$ $\left.\bar{f}\left(V^{1}\right) \in \operatorname{dom} U^{2}\right\} \in U^{1}$. By the inductive assumption, for every $V^{1} \in S$ we have the canonical morphism $\varphi^{V^{1}}: \mathbf{V}^{1} \rightarrow \mathbf{V}^{2}$ where $V^{2}:=\bar{f}\left(V^{1}\right) \in \boldsymbol{\beta}_{<\xi} A^{2}$. We let $\varphi\left(\left\langle V^{1}\right\rangle \frown t\right):=\left\langle\bar{f}\left(V^{1}\right)\right\rangle \frown \varphi^{V^{1}}(t)$ for all $t \in T_{\mathbf{V}^{1}}$. Note that this is the preserving case in the definition of morphism. The verification that $\varphi$ is a morphism of TOUs is routine.

Finally, we prove that $\psi \circ \varphi$ agrees with the canonical morphism $\boldsymbol{\theta}$ of $\mathbf{U}^{1}$ to $\mathbf{U}^{3}$ induced by $h:=g \circ f$, again by induction; we use the notation from the corresponding steps in the first part of this proof.

The case when $U^{1} \in \boldsymbol{\beta}_{0} A^{1}$ is clear. If not, we have

Case (i): $W$ is principal, generated by $U^{2}$. Then $\overline{g \circ f}\left[U^{1}\right]=\bar{g}[W]$ is principal, generated by $U^{3}=\bar{g}\left(U^{2}\right) \in \boldsymbol{\beta}_{<\xi} A^{3}$. We see that, for all $V^{1} \in S$ and $t \in T_{\mathbf{V}^{1}}$, $\boldsymbol{\psi}\left(\varphi\left(\left\langle V^{1}\right\rangle \frown t\right)\right)=\boldsymbol{\psi}\left(\varphi^{V^{1}}(t)\right)=\boldsymbol{\theta}^{V^{1}}(t)=\boldsymbol{\theta}\left(\left\langle V^{1}\right\rangle \frown t\right)$ [the penultimate step is by inductive assumption applied to $\left.\varphi^{V^{1}}: \mathbf{V}^{1} \rightarrow \mathbf{U}^{2}, \psi: \mathbf{U}^{2} \rightarrow \mathbf{U}^{3}\right]$.

Case (ii): $W$ is nonprincipal, $W \sim U^{2} \in \boldsymbol{\beta}_{\xi} A^{2}$. There are two subcases, depending on whether $\bar{g}\left[U^{2}\right]$ is principal or not; note that $\bar{g}\left[U^{2}\right] \sim \bar{g}[W]=\bar{g}\left[\bar{f}\left[U^{1}\right]\right]=\bar{h}\left[U^{1}\right]$, so the same case applies to $\bar{h}\left[U^{1}\right]$. We have, for $V^{1} \in S, t \in T_{V^{1}}, \quad \varphi\left(\left\langle V^{1}\right\rangle \frown t\right)=$ $\left\langle\bar{f}\left(V^{1}\right)\right\rangle \frown \varphi^{V^{1}}(t)$, hence, depending on the case that applies to $g$,

$$
\psi\left(\varphi\left(\left\langle V^{1}\right\rangle \frown t\right)\right)= \begin{cases}\psi^{\bar{f}\left(V^{1}\right)}\left(\varphi^{V^{1}}(t)\right) & \text { in the principal case; } \\ \left\langle\bar{g}\left(\bar{f}\left(V^{1}\right)\right)\right\rangle \frown \psi^{\bar{f}\left(V^{1}\right)}\left(\varphi^{V^{1}}(t)\right) & \text { otherwise. }\end{cases}
$$


On the other hand, for $\boldsymbol{\theta}$ we have

$$
\boldsymbol{\theta}\left(\left\langle V^{1}\right\rangle \frown t\right)= \begin{cases}\boldsymbol{\theta}^{V^{1}}(t) & \text { in the principal case; } \\ \left\langle\bar{h}\left(V^{1}\right)\right\rangle \frown \boldsymbol{\theta}^{V^{1}}(t) & \text { otherwise. }\end{cases}
$$

But $\bar{h}\left(V^{1}\right)=\bar{g}\left(\bar{f}\left(V^{1}\right)\right)$ by Proposition 9.4, and $\psi^{\bar{f}\left(V^{1}\right)}\left(\varphi^{V^{1}}(t)\right)=\boldsymbol{\theta}^{V^{1}}(t)$ by the inductive assumption.

\section{Pedigrees.}

In this section we work in the theory SST, unless explicitly stated otherwise.

Definition 10.1 Let $x \in A \in \mathbb{S}_{\alpha}$. An $\alpha$-pedigree for $x$ over $A$ is a sequence $\vec{u}=\left\langle u_{n}: n \leq \nu\right\rangle$ where $\nu \in \omega$ and

(i) every $u_{n}$ is a stratified ultrafilter over $A\left[\mathrm{ie}, u_{n} \in \boldsymbol{\beta}_{\infty} A\right]$

(ii) $u_{0} \sqsubseteq \alpha ; u_{\nu}=x$

(iii) $(\forall n<m \leq \nu)\left(u_{n} \sqsubset_{\alpha} u_{m}\right)$

(iv) $\left(\forall z \in u_{n}\right)\left(z \sqsubset_{\alpha} u_{n+1} \Rightarrow u_{n+1} \in z\right)$, for all $n<\nu$.

The ultrafilter $u_{0}$ is called the $\alpha$-type of $x$ over $A$; we denote it $\operatorname{tp}_{\alpha}(x ; A)$. We also use $\vec{u}^{+}:=\left\langle u_{n}: 0<n \leq \nu\right\rangle$. Pedigree and type mean 0 -pedigree and 0 -type, resp.

The definition as stated is clearly of the form $\mathcal{P}^{\alpha}(x, A)$ [where $\mathcal{P}(x, A)$ is $\mathcal{P}^{0}(x, A)$, but it is useful to note that

(iii) holds iff $\alpha \sqsubset u_{1} \wedge(\forall n, m)\left(1 \leq n<m \leq \nu \Rightarrow u_{n} \sqsubset u_{m}\right)$, for $\nu>0$, and

(iv) holds iff $\left(\forall z \in u_{n}\right)\left(z \sqsubset u_{n+1} \Rightarrow u_{n+1} \in z\right)$, for all $n<\nu$.

The condition (iv) perhaps becomes more meaningful when stated in terms of monads.

Definition 10.2 For $U \in \mathbb{S}_{\alpha}$ we write $x \mathbb{M}_{\alpha} U$ iff $\left(\forall X \in U \cap \mathbb{S}_{\alpha}\right)(x \in X)$.

The class $\mathbb{M}_{\alpha} U=\bigcap\left(U \cap \mathbb{S}_{\alpha}\right)$ is the $\alpha$-monad of $U$.

In general, $\mathbb{M}_{\alpha} U$ is a proper class. Also, it can be empty. Here is a list of some useful elementary facts about monads.

(1) If $U$ is principal and generated by $a$, then $a \in \mathbb{S}_{\alpha}$ and $\mathbb{M}_{\alpha} U=\{a\}$ is a set. Conversely, if $\mathbb{M}_{\alpha} U \cap \mathbb{S}_{\alpha} \neq 0$, then $U$ is principal. Hence $U$ is nonprincipal if and only if $\mathbb{M}_{\alpha} U \cap \mathbb{S}_{\alpha}=0$.

(2) $\alpha \sqsubseteq \beta$ implies $\mathbb{M}_{\alpha} U \supseteq \mathbb{M}_{\beta} U$.

(3) Let $U, V \in \mathbb{S}_{\alpha}$. If $U \sim V$, then $\mathbb{M}_{\alpha} U=\mathbb{M}_{\alpha} V$. If $\mathbb{M}_{\alpha} U \cap \mathbb{M}_{\alpha} V \neq 0$, then $U \sim V$.

The condition (iv) in Definition 10.1 can be restated as:

(iv) If $u_{n} \sqsubseteq \beta \sqsubset u_{n+1}$, then $u_{n+1} \mathbb{M}_{\beta} u_{n}$. 
Proposition 10.3 (SST) Every $x \in A \in \mathbb{S}_{\alpha}$ has at most one $\alpha$-pedigree over $A$. More generally: Let $x \in A \in \mathbb{S}_{\alpha_{0}}, \alpha_{0} \sqsubseteq \alpha_{1}$. Let $\vec{u}=\left\langle u_{n}: n \leq \nu\right\rangle$ be an $\alpha_{0}$-pedigree for $x$ over $A$, and let $\vec{v}=\left\langle v_{m}: m \leq \mu\right\rangle$ be an $\alpha_{1}$-pedigree for $x$ over $A$. Then $\mu \leq \nu$ and $v_{m}=u_{(\nu-\mu)+m}$ for all $m \leq \mu$.

Proof We begin by noticing that $u_{\nu}=x=v_{\mu}$. Let $j$ be the largest integer such that $j \leq \min \{\nu, \mu\}$ and $u_{\nu-i}=v_{\mu-i}$ holds for all $i \leq j$. If $j=\mu$, then $\mu \leq \nu$ and we are done. If $j=\nu$, then $\nu \leq \mu$. As $u_{0} \in \mathbb{S}_{\alpha_{0}} \subseteq \mathbb{S}_{\alpha_{1}}$, we have $u_{0}=u_{\nu-\nu}=v_{\mu-\nu}$, so $v_{\mu-\nu} \in \mathbb{S}_{\alpha_{1}}$ and $\mu-\nu=0$ [if not, $v_{\mu-\nu-1} \sqsubset_{\alpha_{1}} v_{\mu-\nu}$, but this is impossible], hence $\mu=\nu$, and again we are done.

It remains to show that the remaining case $j<\nu, j<\mu$ leads to a contradiction. We consider $u_{\nu-j-1}$ and $v_{\mu-j-1}$. Let us assume that $u_{\nu-j-1} \sqsubseteq v_{\mu-j-1}$. We let $\beta \boxminus v_{\mu-j-1}$ and $\widetilde{u}:=v_{\mu-j}=u_{\nu-j}$, so that $\beta \sqsubset \widetilde{u}$. By clause (iv) in the definition of pedigrees, $\widetilde{u} \mathbb{M}_{\beta} v_{\mu-j-1}$ and also $\widetilde{u} \mathbb{M}_{\beta} u_{\nu-j-1}$. By the property (3) of monads, this implies $u_{\nu-j-1} \sim v_{\mu-j-1}$ and hence [Proposition 9.1] $u_{\nu-j-1}=v_{\mu-j-1}$, a contradiction with the choice of $j$.

The argument for the case $v_{\mu-j-1} \sqsubseteq u_{\nu-j-1}$ is analogous.

Proposition 10.4 (SST) Let $A, B \in \mathbb{S}_{\alpha}, x \in A \cap B$. If there is an $\alpha$-pedigree for $x$ over $A$, then there is an $\alpha$-pedigree for $x$ over $B$.

Proof Let $\vec{u}=\left\langle u_{0}, \ldots, u_{\nu}\right\rangle$ be an $\alpha$-pedigree for $x$ over $A$. Let $C:=A \cap B$.

Claim. $u_{n} \in \boldsymbol{\beta}_{\infty} A / C$, for all $n \leq \nu$.

Proof of Claim. We proceed by induction. The claim is clearly true for $u_{\nu}=x$. Let $\xi$ be the rank of $u_{n}$ and $\eta$ the rank of $u_{n+1}$. Assume that $u_{n+1} \in \boldsymbol{\beta}_{\eta} A / C$; then $u_{n+1} \in \boldsymbol{\beta}_{<\xi} A / C$, as $\xi>\eta$. For $\beta \boxminus u_{n}$ we have $\boldsymbol{\beta}_{<\xi} A / C \in \mathbb{S}_{\beta}$ and $u_{n+1} \mathbb{M}_{\beta} u_{n}$; so $\boldsymbol{\beta}_{<\xi} A / C \in u_{n}$ and $u_{n} \in \boldsymbol{\beta}_{\xi} A / C$.

We now fix $\rho, \sigma \in \mathbb{S}_{\alpha}$ as in the assumptions of Proposition 9.6, and prove that $\bar{\rho} \circ \vec{u}=\left\langle\bar{\rho}\left(u_{0}\right), \ldots, \bar{\rho}\left(u_{\nu}\right)\right\rangle$ is an $\alpha$-pedigree for $x$ over $B$.

Conditions (i) and (ii) from Definition 10.1 are clearly satisfied by $\bar{\rho} \circ \vec{u}$. As $\bar{\rho}\left(u_{n}\right) \sqsubseteq_{\alpha} u_{n}$ and $u_{n}=\bar{\sigma}\left(\bar{\rho}\left(u_{n}\right)\right) \sqsubseteq_{\alpha} \bar{\rho}\left(u_{n}\right)$, we have $u_{n} \boxminus_{\alpha} \bar{\rho}\left(u_{n}\right)$; this implies (iii).

In order to prove (iv), we note that $\bar{\rho}\left(u_{n}\right)=\bar{\rho}\left[u_{n}\right]$ for all $n<\nu$, by the remark following Proposition 9.6. Given $\beta$ such that $u_{n} \sqsubseteq \beta \sqsubset u_{n+1}$, and $Y \sqsubseteq \beta, Y \in \bar{\rho}\left[u_{n}\right]$, there is some $X \in u_{n}$ such that $\bar{\rho}[X] \subseteq Y$. By Transfer into $\mathbb{S}_{\beta}$, we can assume $X \in \mathbb{S}_{\beta}$. Hence $u_{n+1} \in X$ [by (iv) for $\left.\vec{u}\right]$ and $\bar{\rho}\left(u_{n+1}\right) \in \bar{\rho}[X] \subseteq Y$. This shows (iv) holds for $\bar{\rho} \circ \vec{u}$. 
Proposition 10.5 (SST ${ }^{*}$ ) Let $\vec{u}$ be the $\alpha$-pedigree for $x$ over $A \in \mathbb{S}_{\alpha}$.

If $\alpha \sqsubseteq \beta \sqsubset x$, then there is a unique $n<\nu$ such that $u_{n} \sqsubseteq \beta \sqsubset u_{n+1}$. The sequence $\vec{u}\left\lceil\beta:=\left\langle u_{n}, u_{n+1}, \ldots, u_{\nu}\right\rangle\right.$ is then the $\beta$-pedigree for $x$ over $A$. If $x \sqsubseteq \beta$, we let $\vec{u} \uparrow \beta:=\left\langle u_{\nu}\right\rangle=\langle x\rangle$; it is again the $\beta$-pedigree for $x$ over $A$.

Proof The set $\operatorname{ran} \vec{u}=\left\{u_{0}, \ldots, u_{\nu}\right\}$ is a level set by Proposition 8.6. Hence $\sqsubseteq\lceil\operatorname{ran} \vec{u}$ is a well-ordering, and there is a least $n$ such that $u_{n} \sqsubseteq \beta \sqsubset u_{n+1}$.

Corollary 10.6 (SST $\left.{ }^{*}\right)$ Let $A \in \mathbb{S}_{\alpha}$ and $\alpha \sqsubseteq \beta$; if $\operatorname{tp}_{\beta}(x, A)=\operatorname{tp}_{\beta}(y, A)$, then $\operatorname{tp}_{\alpha}(x, A)=\operatorname{tp}_{\alpha}(y, A)$.

Proof Let $\vec{u}=\left\langle u_{0}, \ldots, u_{\nu}\right\rangle$ and $\vec{v}=\left\langle v_{0}, \ldots, v_{\mu}\right\rangle$ be the $\alpha$-pedigrees for $x$ and $y$ over $A$, resp., and let $u_{n} \sqsubseteq \beta \sqsubset u_{n+1}, v_{m} \sqsubseteq \beta \sqsubset u_{m+1}$. Then $u_{n}=\operatorname{tp}_{\beta}(x, A)=$ $\operatorname{tp}_{\beta}(y, A)=v_{m}$. It is easy to check that the sequence $\left\langle u_{0}, \ldots, u_{n}=v_{m}, \ldots, v_{\mu}\right\rangle$ is an $\alpha$-pedigree for $y$ over $A$. By Proposition 10.3 now $v_{0}=u_{0}$.

Theorem $10.7\left(\right.$ SST $\left.^{*}\right)$ If $x \in A \in \mathbb{S}_{\alpha}$, then an $\alpha$-pedigree for $x$ over $A$ exists.

Proof Let $\mathcal{P}^{\alpha}(x)$ be the statement "For every $A \in \mathbb{S}_{\alpha}$, if $x \in A$, then there exists an $\alpha$-pedigree for $x$ over $A$." By Proposition $10.4, \mathcal{P}^{\alpha}(x)$ is equivalent to "For some $A \in \mathbb{S}_{\alpha}$ such that $x \in A$, there exists an $\alpha$-pedigree for $x$ over $A$." Our goal is to prove that $\mathcal{P}^{\alpha}(x)$ holds for all $\alpha$.

$\mathcal{P}^{\alpha}(x)$ holds for all $\alpha \sqsupseteq x$, because $\left\langle u_{0}\right\rangle$ with $u_{0}=x$ is then clearly an $\alpha$-pedigree for $x$ over $A$.

By Granularity, there is a least $\alpha$ for which $\mathcal{P}^{\alpha}(x)$ holds. Let us assume $0 \sqsubset \alpha$. We fix $A \in \mathbb{S}_{0}$ such that $x \in A$, and an $\alpha$-pedigree $\vec{u}=\left\langle u_{n}: n \leq \nu\right\rangle$ for $x$ over $A$, and obtain a contradiction by showing that there is a $\beta$-pedigree for $x$ over $A$ for some $\beta \sqsubset \alpha$. If $u_{0} \in \mathbb{S}_{0}$, then $\vec{u}$ is also a 0 -pedigree for $x$ over $A$, and we let $\beta=0$.

Otherwise we fix $\bar{\xi} \in \mathbb{S}_{0}$ such that $u_{0} \in \boldsymbol{\beta}_{<\bar{\xi}} A$. Let $W:=W_{u_{0}, \boldsymbol{\beta}_{<\bar{\xi}} A}$ be the principal ultrafilter generated by $u_{0}$; we note $W \boxminus u_{0} \sqsupset 0$. By Block Standardization, there is $u^{\prime} \sqsubset u_{0}$ such that $(\forall X \sqsubset \alpha)\left(X \in u^{\prime} \Leftrightarrow X \in W\right)$; we let $\beta \boxminus u^{\prime}$. Then $u^{\prime} \in \mathbb{S}_{\beta}$ is an ultrafilter over $\boldsymbol{\beta}_{<\bar{\xi}} A, u_{0} \mathbb{M}_{\gamma} u^{\prime}$ for all $\beta \sqsubseteq \gamma \sqsubset \alpha$, and $u^{\prime}$ is nonprincipal [because $u_{0} \notin \mathbb{S}_{\beta}$ ]. By Proposition 9.2, $u^{\prime} \sim u$ for some $u \in \boldsymbol{\beta}_{\bar{\xi}} A \cap \mathbb{S}_{\beta}$. Then also $u_{0} \mathbb{M}_{\gamma} u$ for all $\beta \sqsubseteq \gamma \sqsubset \alpha$, and the sequence $\langle u\rangle \frown \vec{u}$ is a $\beta$-pedigree for $x$ over $A$, a contradiction. Hence $\alpha \boxminus 0$ and $\mathcal{P}^{0}(x)$ holds. Proposition 10.5 now implies that $\mathcal{P}^{\alpha}(x)$ holds for all levels $\alpha$. 
Definition 10.8 An $\alpha$-pedigree $\vec{u}$ for $x$ over $A$ is a $\operatorname{good} \alpha$-pedigree if, for $u:=$ $\operatorname{tp}_{\alpha}(x ; A), \vec{u}^{+} \mathbb{M}_{\alpha}(\Sigma \mathbf{u})$ and

$$
f \sqsubseteq \mathbf{u} g \Leftrightarrow f\left(\vec{u}^{+}\right) \sqsubseteq_{\alpha} g\left(\vec{u}^{+}\right) \quad \text { for all } f, g \in \mathbb{V}^{\Sigma} \mathbf{u} \cap \mathbb{S}_{\alpha} .
$$

An equivalent statement of the property (j) is that $\mathfrak{j}_{\alpha ; x, A}: \mathbb{V}^{\Sigma \mathbf{u}} \cap \mathbb{S}_{\alpha} \rightarrow \mathbb{S}_{\alpha}\left[\vec{u}^{+}\right]$, defined by $\mathfrak{j}_{\alpha ; x, A}(f)=f\left(\vec{u}^{+}\right)$, is an isomorphism of interpretations $[\mathcal{U} \ell t(\mathbb{V} ; \Sigma \mathbf{u})]^{\mathbb{S}_{\alpha}}$ and $\left(\mathbb{S}_{\alpha}\left[\vec{u}^{+}\right],=, \in, \sqsubseteq_{\alpha}\right)$ [see Theorem 3.7]. Of course, $\mathbb{S}_{\alpha}\left[\vec{u}^{+}\right]=\mathbb{S}_{\alpha}[\vec{u}]$, because $u \in \mathbb{S}_{\alpha}$. Also $\mathbb{S}_{\alpha}[\vec{u}]=\mathbb{S}_{\alpha}[\operatorname{ran} \vec{u}]$, because $u_{n} \sqsubset_{\alpha} u_{m}$ iff rank $u_{n}>$ rank $u_{m}$, and so the sequence $\left\langle u_{0}, \ldots, u_{\nu}\right\rangle$ is $\in$-definable from the set $\left\{u_{0}, \ldots, u_{\nu}\right\}$.

Theorem 10.9 (SST ${ }^{*}$ ) For any $x \in A \in \mathbb{S}_{\alpha}$, the $\alpha$-pedigree for $x$ over $A$ is good.

Proof We fix an $\alpha_{0}$-pedigree $\vec{u}=\left\langle u_{0}, \ldots, u_{\nu}\right\rangle$ for $x$ over $A \in \mathbb{S}_{\alpha_{0}}$, and use Granularity to prove that $\alpha_{0}$ is the least level $\alpha \sqsupseteq \alpha_{0}$ for which $\vec{u}\lceil\alpha$ is $\alpha$-good. We write $\mathfrak{j}_{\alpha}$ for $\mathfrak{j}_{\alpha ; x, A}$.

If $\alpha \sqsupseteq x$, then all is trivial: $\vec{u}\left\lceil\alpha=\left\langle u_{\nu}\right\rangle\right.$ where $u_{\nu}=x=\operatorname{tp}_{\alpha}(x ; A)=: u, \vec{u}^{+}=0$, $\Sigma \mathbf{u}=\{\{0\}\}$ is the principal ultrafilter over $\{0\}, \mathbb{S}_{\alpha}\left[\vec{u}^{+}\right]=\mathbb{S}_{\alpha}, \sqsubseteq_{\alpha}$ is the identity on $\mathbb{S}_{\alpha}$, and for any $f=\{(0, a)\} \in \mathbb{S}_{\alpha}, \mathfrak{j}_{\alpha}(f)=f\left(\vec{u}^{+}\right)=f(0)=a$; evidently, $\mathfrak{j}_{\alpha}$ preserves $\sqsubseteq_{\alpha}$.

Let $\alpha \sqsupseteq \alpha_{0}$ be the least level such that $\vec{u}\lceil\alpha$ is $\alpha$-good. Then $\alpha \sqsubset x$, there is a unique $n<\nu$ such that $u_{n} \sqsubseteq \alpha \sqsubset u_{n+1}$, and $\vec{u}\left\lceil\alpha=\left\langle u_{n}, u_{n+1}, \ldots, u_{\nu}\right\rangle\right.$. If $\alpha \boxminus \alpha_{0}$, we are done; otherwise, we obtain a contradiction by showing that $\vec{u} \uparrow \beta$ is $\beta$-good, for some $\beta \sqsubset \alpha$. There are two cases to consider.

Case 1. $u_{n} \sqsubset \alpha$.

Then, for every $u_{n} \sqsubseteq \beta \sqsubset \alpha, \vec{u} \uparrow \alpha=\vec{u} \uparrow \beta$ is also the $\beta$-pedigree for $x$ over $A$ and $u:=\operatorname{tp}_{\beta}(x ; A)=\operatorname{tp}_{\alpha}(x ; A)=u_{n}$.

By the inductive assumption, $(\vec{u} \uparrow \alpha)^{+} \mathbb{M}_{\alpha} \Sigma \mathbf{u}$, hence also $(\vec{u} \uparrow \beta)^{+} \mathbb{M}_{\beta} \Sigma \mathbf{u}$, and $f \sqsubseteq \mathbf{u} g \Leftrightarrow f\left(\vec{u}^{+}\right) \sqsubseteq_{\alpha} g\left(\vec{u}^{+}\right)$for all $f, g \in \mathbb{V}^{\Sigma} \mathbf{u} \cap \mathbb{S}_{\beta}$. It remains to show that the last statement holds with $\sqsubseteq_{\alpha}$ replaced by $\sqsubseteq_{\beta}$.

It suffices to prove that, for $f \in \mathbb{S}_{\beta}, f\left(\left(\vec{u}\lceil\beta)^{+}\right) \sqsubseteq_{\alpha} 0\right.$ implies $f\left((\vec{u} \uparrow \beta)^{+}\right) \sqsubseteq_{\beta} 0$. [Indeed, from $\left(z \sqsubseteq_{\alpha} 0 \Rightarrow z \sqsubseteq_{\beta} 0\right)$ it follows that, for all $y, z \sqsubseteq_{\alpha} y \Leftrightarrow z \sqsubseteq_{\alpha} 0 \vee z \sqsubseteq$ $y \Leftrightarrow z \sqsubseteq_{\beta} 0 \vee z \sqsubseteq y \Leftrightarrow z \sqsubseteq_{\beta} y$.]

But $f\left(\left(\vec{u}\lceil\beta)^{+}\right) \sqsubseteq_{\alpha} 0\right.$ means [apply $\mathfrak{j}_{\alpha}^{-1}$ ] that $\mathcal{U} \ell t(\mathbb{V} ; \mathbf{u}) \vDash f=\mathfrak{k}_{\Sigma \mathbf{u}}(c)$, for some $c \in$ $\mathbb{S}_{\alpha}$. As $f \in \mathbb{S}_{\beta}$, we have $c \in \mathbb{S}_{\beta}$, hence $f\left(\left(\vec{u}\lceil\beta)^{+}\right)=c \in \mathbb{S}_{\beta}\right.$, ie, $f\left(\left(\vec{u}\lceil\beta)^{+}\right) \sqsubseteq_{\beta} 0\right.$. 
Case 2. $u_{n} \boxminus \alpha$.

Then $n>0$ and we consider all $\beta \sqsupseteq \alpha_{0}$ such that $u_{n-1} \sqsubseteq \beta \sqsubset u_{n}$. We have $\vec{u}\left\lceil\beta=\left\langle u_{n-1}, u_{n}, \ldots, u_{\nu}\right\rangle=\left\langle u_{n-1}\right\rangle \frown \vec{u}\left\lceil\alpha, \quad\left(\vec{u}\lceil\beta)^{+}=\vec{u}\lceil\alpha\right.\right.\right.$, and $u:=$ $\operatorname{tp}_{\beta}(x ; A)=u_{n-1}$. We note that $u_{n} \mathbb{M}_{\gamma} u$ for all $\beta \sqsubseteq \gamma \sqsubset \alpha$; by the inductive assumption, also $(\vec{u} \uparrow \alpha)^{+} \mathbb{M}_{\alpha} \Sigma \mathbf{u}_{n}$.

We first prove that $\left(\vec{u}\lceil\beta)^{+} \mathbb{M}_{\beta} \Sigma \mathbf{u}\right.$.

By Definitions 9.8 and 6.2, $\Sigma \mathbf{u}=\Sigma_{u}\left(\Sigma \mathbf{u}^{\prime}\right)$, where $u^{\prime}$ ranges over the domain of $u$. Let $X \in \mathbb{S}_{\beta}, X \subseteq \operatorname{dom} \Sigma \mathbf{u}=\bigcup_{u^{\prime} \in u}\left\langle u^{\prime}\right\rangle \frown \operatorname{dom} \Sigma \mathbf{u}^{\prime}$, and $X \in \Sigma \mathbf{u}$; then $Y:=\left\{u^{\prime} \in \operatorname{dom} u:(X)_{\left\langle u^{\prime}\right\rangle} \in \Sigma \mathbf{u}^{\prime}\right\} \in u$. As $Y \in \mathbb{S}_{\beta}$ and $u_{n} \mathbb{M}_{\beta} u$, we get $u_{n} \in Y$, ie, $(X)_{\left\langle u_{n}\right\rangle} \in \Sigma \mathbf{u}_{n}$. As $u_{n} \in \mathbb{S}_{\alpha},(X)_{\left\langle u_{n}\right\rangle} \in \mathbb{S}_{\alpha}$, and so $(\vec{u} \uparrow \alpha)^{+} \in(X)_{\left\langle u_{n}\right\rangle}$, ie, $\left(\vec{u}\lceil\beta)^{+}=\left\langle u_{n}\right\rangle \frown\left(\vec{u}\lceil\alpha)^{+} \in X\right.\right.$. This proves $\left(\vec{u}\lceil\beta)^{+} \mathbb{M}_{\beta} \Sigma \mathbf{u}\right.$.

By the inductive assumption we have the isomorphism $\mathfrak{j}_{\alpha}$ of $\left[\mathcal{U} \ell t\left(\mathbb{V} ; \mathbf{u}_{n}\right)\right]^{\mathbb{S}_{\alpha}}$ and $\left(\mathbb{S}_{\alpha}\left[(\vec{u} \uparrow \alpha)^{+}\right],=, \in, \sqsubseteq_{\alpha}\right)$.

From $\mathbb{S}_{\beta}\left[u_{n}\right] \preccurlyeq \mathbb{S}_{\alpha}$ we get that $j_{\alpha}$, restricted to $\mathbb{S}_{\beta}\left[u_{n}\right]$, is an isomorphism of $\left[\mathcal{U} \ell t\left(\mathbb{V} ; \mathbf{u}_{n}\right)\right]^{\mathbb{S}_{\beta}\left[u_{n}\right]}$ and $\left(\mathbb{S}_{\beta}\left[u_{n}\right]\left[(\vec{u} \uparrow \alpha)^{+}\right],=, \in, \sqsubseteq_{\alpha}\right)=\left(\mathbb{S}_{\beta}\left[(\vec{u} \uparrow \beta)^{+}\right],=, \in, \sqsubseteq_{\alpha}\right)$ [the ultrapower is defined because $\left.u_{n} \in \mathbb{S}_{\beta}\left[u_{n}\right]\right]$.

We also have the isomorphism $j$ of $[\mathcal{U} \ell(\mathbb{V} ; u)]^{\mathbb{S}_{\beta}}$ and $\left(\mathbb{S}_{\beta}\left[u_{n}\right],=, \in\right) \preccurlyeq\left(\mathbb{S}_{\alpha},=, \in\right)$ given by $\mathfrak{j}(f)=f\left(u_{n}\right)$; especially, $\mathfrak{j}\left(I d_{\operatorname{dom} u}\right)=u_{n}$ [Theorem 3.7].

$\mathfrak{j}$ induces an isomorphism [also denoted $\mathfrak{j}$ ] of

$$
\left[\mathcal{U} \ell t\left(\mathbb{V} ; j^{-1}\left(\mathbf{u}_{n}\right)\right)\right]^{[\mathcal{U} \ell(\mathbb{V} ; u)]^{\mathbb{S}_{\beta}}} \quad \text { and } \quad\left[\mathcal{U} \ell t\left(\mathbb{V} ; \mathbf{u}_{n}\right)\right]^{\mathbb{S}_{\beta}\left[u_{n}\right]} .
$$

Observing that $[\mathcal{U} \ell(\mathbb{V} ; u)]^{\mathbb{S}_{\beta}}=\left(\mathbb{V}^{u} \cap \mathbb{S}_{\beta}, \ldots\right)$ and $I d_{\operatorname{dom} u} \in \mathbb{S}_{\beta}$, and using $\mathfrak{j}^{-1}\left(u_{n}\right)=$ $I d_{\operatorname{dom} u}=\left\langle u^{\prime}: u^{\prime} \in \operatorname{dom} u\right\rangle$, we get $j^{-1}\left(\mathbf{u}_{n}\right)=\left\langle\mathbf{u}^{\prime}: u^{\prime} \in \operatorname{dom} u\right\rangle=\left\langle\mathbf{u}_{\left\langle u^{\prime}\right\rangle}: u^{\prime} \in\right.$ $\operatorname{dom} u\rangle$ [recall the definition of $\mathbf{u}$, the TOU associated to $u$ ].

If $T^{(1)}=\{0\} \cup\left\{\left\langle u^{\prime}\right\rangle: u^{\prime} \in \operatorname{dom} u\right\}$ denotes the $\mathbf{u}$-subtree of $T_{\mathbf{u}}$ of level 1, we have further $\left\langle\mathbf{u}_{\left\langle u^{\prime}\right\rangle}: u^{\prime} \in \operatorname{dom} u\right\rangle=\left\langle\left(\mathbf{u} / T^{(1)}\right)\left(u^{\prime}\right): u^{\prime} \in \operatorname{dom} u\right\rangle$. [For the last step, recall that $\Sigma T^{(1)}$ is identified with $\operatorname{dom} \mathbf{u}(0)=\operatorname{dom} u$ via the map $\left\langle u^{\prime}\right\rangle \mapsto u^{\prime}$.]

We can thus write $\mathbf{u} / T^{(1)}$ for $\mathfrak{j}^{-1}\left(\mathbf{u}_{n}\right)$ in $(*)$. Furthermore, by the Factoring Theorem, $\Omega_{T^{(1)}, \mathbf{u}}$ [restricted to $\mathbb{S}_{\beta}$ ] is an isomorphism of $\left(\mathcal{U} \ell(\mathbb{V} ; \Sigma \mathbf{u}), \sqsubseteq_{\mathbf{u} ; I T_{T^{(1)}}}\right)^{\mathbb{S}_{\beta}}$ and $\left[\left[\mathcal{U} \ell t\left(\mathbb{V} ; \mathbf{u} / T^{(1)}\right)\right]^{\mathcal{U} \ell(\mathbb{V} ; u)}\right]^{\mathbb{S}_{\beta}}=\left[\mathcal{U} \ell t\left(\mathbb{V} ; \mathbf{u} / T^{(1)}\right)\right]^{[\mathcal{U} \ell(\mathbb{V} ; u)]^{\mathbb{S}_{\beta}}}$.

The composition $\Psi:=j_{\alpha} \circ j \circ \Omega_{T^{(1)}, \mathbf{u}}$ is an isomorphism of $\left(\mathcal{U} \ell(\mathbb{V} ; \Sigma \mathbf{u}), \sqsubseteq_{\mathbf{u} ; I d_{T^{(1)}}}\right)^{\mathbb{S}_{\beta}}$ and $\left(\mathbb{S}_{\beta}\left[(\vec{u} \uparrow \beta)^{+}\right],=, \in, \sqsubseteq_{\alpha}\right)$; it remains to show that $\Psi=\mathfrak{j}_{\beta ; x, A}$, and that, for $f, g \in \mathbb{V}^{\Sigma} \mathbf{u} \cap \mathbb{S}_{\beta}, f \sqsubseteq_{\mathbf{u}} g \Leftrightarrow \Psi(f) \sqsubseteq_{\beta} \Psi(g)$. 
Let $f \in \mathbb{V}^{\Sigma} \mathbf{u} \cap \mathbb{S}_{\beta}$; we show that $\Psi(f)=f\left(\left(\vec{u}\lceil\beta)^{+}\right)\right.$. First, $\Omega_{T^{(1)}, \mathbf{u}}(f)=f / T^{(1)}=$ $\left\langle f_{\left\langle u^{\prime}\right\rangle}: u^{\prime} \in \operatorname{dom} u\right\rangle$, where $u$-almost everywhere $\operatorname{dom} f_{\left\langle u^{\prime}\right\rangle} \in \Sigma \mathbf{u}_{\left\langle u^{\prime}\right\rangle}$ and $f_{\left\langle u^{\prime}\right\rangle}(t)=$ $f\left(\left\langle u^{\prime}\right\rangle \frown t\right)$. Hence $[\mathcal{U} \ell(\mathbb{V} ; u)]^{\mathbb{S}_{\beta}} \vDash \cdot \operatorname{dom} f / T^{(1)} \in \Sigma \mathbf{u} / T^{(1)}$ ", so, second, $\mathfrak{j}\left(f / T^{1}\right)=$ $\left(f / T^{(1)}\right)\left(u_{n}\right)=f_{\left\langle u_{n}\right\rangle} \in \mathbb{S}_{\beta}\left[u_{n}\right]$ and $\mathbb{S}_{\beta}\left[u_{n}\right] \vDash$ "dom $f_{\left\langle u_{n}\right\rangle} \in \Sigma \mathbf{u}_{n}$ ", hence also [as $\left.\mathbb{S}_{\beta}\left[u_{n}\right] \preccurlyeq \mathbb{S}_{\alpha}\right], \mathbb{S}_{\alpha} \vDash \cdot \operatorname{dom} f_{\left\langle u_{n}\right\rangle} \in \Sigma \mathbf{u}_{n}$ ". Finally, $\Psi(f)=\mathfrak{j}_{\alpha}\left(f_{\left\langle u_{n}\right\rangle}\right)=f_{\left\langle u_{n}\right\rangle}((\vec{u} \uparrow$ $\left.\alpha)^{+}\right)=f\left(\left\langle u_{n}\right\rangle \frown\left(\vec{u}\lceil\alpha)^{+}\right)=f\left(\left(\vec{u}\lceil\beta)^{+}\right)\right.\right.$.

This argument also shows that if $f==_{\Sigma \mathbf{u}} \mathfrak{k}_{\Sigma \mathbf{u}}(c)$, then $\Psi(f)=c \in \mathbb{S}_{\beta}$, and if $f \neq \neq_{\Sigma \mathbf{u}}$ $\mathfrak{k}_{\Sigma \mathbf{u}}(c)$ for any $c$, then $\Psi(f) \notin \mathbb{S}_{\beta}$. That is, $\Psi$ preserves the level $0: f \sqsubseteq_{\mathbf{u}} \mathfrak{k}_{\Sigma \mathbf{u}}(0)$ if and only if $\Psi(f) \sqsubseteq_{\beta} 0$. Moreover, since the last equivalence is true for all $\beta^{\prime}$ such that $\beta \sqsubseteq \beta^{\prime} \sqsubset \alpha$, we have $f \sqsubseteq_{\mathbf{u}} \mathfrak{k}_{\Sigma \mathbf{u}}(0)$ if and only if $\Psi(f) \sqsubseteq_{\alpha} 0$.

If $\mathfrak{k}_{\Sigma \mathbf{u}}(0) \sqsubset_{\mathbf{u}} f, g$, then $f \sqsubseteq_{\mathbf{u}} g$ iff $\Psi(f) \sqsubseteq_{\alpha} \Psi(g)$ iff $\Psi(f) \sqsubseteq_{\alpha} 0 \vee \Psi(f) \sqsubseteq \Psi(g)$ iff $\Psi(f) \sqsubseteq_{\beta} 0 \vee \Psi(f) \sqsubseteq \Psi(g)$ iff $\Psi(f) \sqsubseteq_{\beta} \Psi(g)$.

Theorem 10.10 (SST*) Let $\vec{u}$ be the $\alpha$-pedigree for $x$ over $A$ and let $\vec{v}$ be the $\alpha$-pedigree for $y$ over $B$, where $y=f(x)$ and $A, B, f \in \mathbb{S}_{\alpha}, f: A \rightarrow B$. Let $u:=\operatorname{tp}_{\alpha}(x ; A)$ and $v:=\operatorname{tp}_{\alpha}(y ; B)$ Then $v=\bar{f}(u)$ and $\operatorname{ran} \vec{v}=\bar{f}[\operatorname{ran} \vec{u}]$.

If $\varphi: \mathbf{u} \rightarrow \mathbf{v}$ is the morphism canonically induced by $\bar{f}$, then the following diagram commutes.

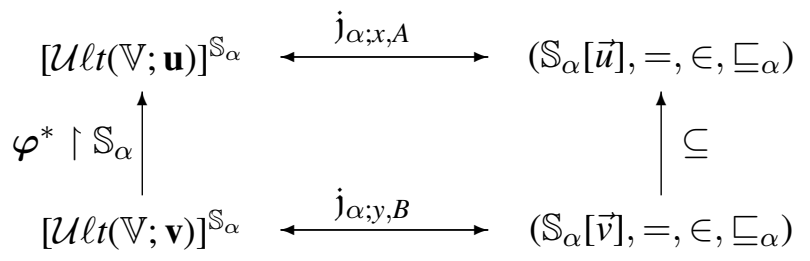

Proof We fix $\alpha_{0}$-pedigrees $\vec{u}$ for $x$ over $A$ and $\vec{v}$ for $y$ over $B$, where $y=f(x)$ and $A, B, f \in \mathbb{S}_{\alpha_{0}}$, and prove that $\alpha_{0}$ is the least level $\alpha \sqsupseteq \alpha_{0}$ such that the assertions hold for $\vec{u} \uparrow \alpha$ and $\vec{v} \uparrow \alpha$.

If $x \sqsubseteq \alpha$, then also $y \sqsubseteq \alpha, \quad u:=\operatorname{tp}_{\alpha}(x ; A)=x, \quad v:=\operatorname{tp}_{\alpha}(y ; B)=y, \quad v=\bar{f}(u)$, $\Sigma \mathbf{u}=\Sigma \mathbf{v}=\{\{0\}\}, \varphi(0)=0, \varphi^{*}=\mathbb{I} d$, and $\mathbb{S}_{\alpha}[\vec{u}]=\mathbb{S}_{\alpha}[\vec{v}]=\mathbb{S}_{\alpha}$. The diagram commutes trivially.

By Granularity, there is a least $\alpha \sqsupseteq \alpha_{0}$ such that the assertion is true for $\vec{u}\lceil\alpha$ and $\vec{v}\left\lceil\alpha\right.$. We assume that $\alpha \sqsupset \alpha_{0}$ and obtain a contradiction. Let $u_{n} \sqsubseteq \alpha \sqsubset u_{n+1}$.

Case 1. $u_{n} \sqsubset \alpha$.

We consider any $\beta \sqsupseteq \alpha_{0}$ such that $u_{n} \sqsubseteq \beta \sqsubset \alpha$. Then $u:=\operatorname{tp}_{\beta}(x ; A)=\operatorname{tp}_{\alpha}(x ; A)=u_{n}$ and $\bar{f}(u) \sqsubseteq \beta$, so $\vec{u} \uparrow \beta=\vec{u} \uparrow \alpha$ and $\vec{v} \uparrow \beta=\vec{v} \uparrow \alpha$ are $\beta$-pedigrees and $v:=$ 
$\mathbf{t p}_{\beta}(y ; B)=\mathbf{t p}_{\alpha}(y ; B)$. The diagram commutes for $\alpha$, by the inductive assumption. We have to prove that it commutes with $\beta$ in place of $\alpha$. As $\mathfrak{j}_{\beta ; x, A}(f)=f\left(\left(\vec{u}\lceil\beta)^{+}\right)=\right.$ $f\left(\left(\vec{u}\lceil\alpha)^{+}\right)=\mathfrak{j}_{\alpha ; x, A}(f)\right.$, we have $\mathfrak{j}_{\beta ; x, A}=\mathfrak{j}_{\alpha ; x, A}\left\lceil\mathbb{S}_{\beta}\right.$; similarly $\mathfrak{j}_{\beta ; y, B}=\mathfrak{j}_{\alpha ; y, B}\left\lceil\mathbb{S}_{\beta}\right.$. As $\operatorname{ran}(\vec{v} \uparrow \beta)=\bar{f}[\operatorname{ran}(\vec{u} \uparrow \beta)]$ and $\bar{f} \in \mathbb{S}_{\beta}$, we have $\mathbb{S}_{\beta}[\vec{v} \uparrow \beta]=\mathbb{S}_{\beta}[\operatorname{ran}(\vec{v} \uparrow$ $\beta)] \subseteq \mathbb{S}_{\beta}\left[\operatorname{ran}(\vec{u}\lceil\beta)]=\mathbb{S}_{\beta}[\vec{u}\lceil\beta]\right.$. The morphism $\varphi: \mathbf{u} \rightarrow \mathbf{v}$ is $\in$-definable from $A, B, f \in \mathbb{S}_{\beta}$, and so it belongs to $\mathbb{S}_{\beta}$. It follows, by Transfer between $\mathbb{S}_{\alpha}$ and $\mathbb{S}_{\beta}$, that $\varphi^{*}\left\lceil\mathbb{S}_{\beta}\right.$ is a morphism of $[\mathcal{U} \ell t(\mathbb{V} ; \mathbf{u})]^{\mathbb{S}_{\beta}}$ into $[\mathcal{U} \ell t(\mathbb{V} ; \mathbf{v})]^{\mathbb{S}_{\beta}}$. The commutativity of the diagram is of course preserved.

Case 2. $u_{n} \boxminus \alpha$.

Then $n>0$ and we consider any $\beta \sqsupseteq \alpha_{0}$ such that $u_{n-1} \sqsubseteq \beta \sqsubset u_{n}$, so $u:=$ $\operatorname{tp}_{\beta}(a ; A)=u_{n-1}$. Let $v_{0}:=\operatorname{tp}_{\alpha}(y ; B)$.

Subcase A: $v_{0} \in \mathbb{S}_{\beta}$.

Claim. Then $\bar{f}(u)=v_{0}$.

Proof of Claim.

According to Definition 9.3 of $\bar{f}(u)$, one considers $w:=\bar{f}_{<\xi}[u]$ for the appropriate $\xi$. If $Y \in w \cap \mathbb{S}_{\beta}$, then $\bar{f}_{<\xi}^{-1}[Y] \in u \cap \mathbb{S}_{\beta}$, hence $u_{n} \in \bar{f}_{<\xi}^{-1}[Y]$ and $v_{0}=\bar{f}\left(u_{n}\right) \in Y$. We conclude that $w$ is a principal ultrafilter generated by $v_{0}$, and $\bar{f}(u)=\mathfrak{m}(w)=v_{0}$.

Returning to the proof of Subcase A, we have $\bar{f}(u)=v_{0}$ as well as $\bar{f}\left(u_{n}\right)=v_{0}$, $\vec{v} \uparrow \alpha=\vec{v}\left\lceil\beta\right.$, and $v:=v_{0}=\operatorname{tp}_{\beta}(y ; B)$. As in Case $1, \operatorname{ran}(\vec{v}\lceil\beta)=\bar{f}[\operatorname{ran}(\vec{u}\lceil\beta)]$, $\mathbb{S}_{\beta}\left[\vec{v}\lceil\beta] \subseteq \mathbb{S}_{\beta}\left[\vec{u}\lceil\beta]\right.\right.$, and $\mathfrak{j}_{\beta ; y, B}$ is the restriction of $\mathfrak{j}_{\alpha ; y, B}$ to $\mathbb{S}_{\beta}$. In the definition of the induced canonical morphism $\varphi: u \rightarrow v$ [proof of Proposition 9.9] this is the case (i); ie, for $u$-almost all $u^{\prime}, \varphi\left(\left\langle u^{\prime}\right\rangle\right)=0$ and $\varphi_{\left\langle u^{\prime}\right\rangle}=\varphi^{u^{\prime}}$, where $\varphi^{u^{\prime}}: u^{\prime} \rightarrow v$ are the induced canonical morphisms. Consider $g \in \mathbb{V}^{\Sigma \mathbf{v}} \cap \mathbb{S}_{\beta}$. On the one hand,

$$
\mathfrak{j}_{\beta ; y, B}(g)=\mathfrak{j}_{\alpha ; y, B}(g)=\mathfrak{j}_{\alpha ; x, A}\left(\left(\varphi^{u_{n}}\right)^{*}(g)\right)
$$

by the inductive assumption.

On the other hand, $\varphi^{*}(g)\left(\left\langle u^{\prime}\right\rangle \frown t\right)=g\left(\varphi\left(\left\langle u^{\prime}\right\rangle \frown t\right)\right)=g\left(\varphi^{u^{\prime}}(t)\right)=\left(\varphi^{u^{\prime}}\right)^{*}(g)(t)$ $u$-almost everywhere, so [proof of Theorem 10.9] $\Omega_{T^{(1)}, \mathbf{u}}\left(\varphi^{*}(g)\right)\left(u^{\prime}\right)=\left(\varphi^{u^{\prime}}\right)^{*}(g)$, $\mathfrak{j}\left(\Omega_{T^{(1)}, \mathbf{u}}\left(\varphi^{*}(g)\right)\right)=\left(\varphi^{u_{n}}\right)^{*}(g)$, and

$$
\mathfrak{j}_{\beta ; x, A}\left(\varphi^{*}(g)\right)=\mathfrak{j}_{\alpha ; x, A}\left(\left(\varphi^{u_{n}}\right)^{*}(g)\right) .
$$

Comparing (*) and (**) gives $\mathfrak{j}_{\beta ; y, B}(g)=\mathfrak{j}_{\beta ; x, A}\left(\varphi^{*}(g)\right)$, ie, the commutativity of the diagram for $\beta$.

Subcase B. $v_{0} \in \mathbb{S}_{\alpha} \backslash \mathbb{S}_{\beta}$. 
We let $v:=\bar{f}(u)$; then $v \in \mathbb{S}_{\beta} \cap \boldsymbol{\beta}_{\infty} B$.

Claim. $v_{0} \mathbb{M}_{\beta} v$.

Proof of Claim. $u \in \boldsymbol{\beta}_{\xi} A, v \in \boldsymbol{\beta}_{\eta} B$, for suitable $\xi, \eta \in \mathbb{S}_{\beta}$. Let $w=\bar{f}_{<\xi}[u]$; from $u_{n} \mathbb{M}_{\beta} u$ we get $v_{0}=\bar{f}\left(u_{n}\right) \mathbb{M}_{\beta} w$ [Proposition 3.5], so $w$ is nonprincipal [ $v_{0} \notin \mathbb{S}_{\beta}$ ], $w \sim \bar{f}(u)$ by definition of the latter, and, consequently, $v_{0} \mathbb{M}_{\beta} v$.

It follows that $\vec{v}\lceil\beta=\langle v\rangle \frown \vec{v}\lceil\alpha$. As in Case 1 now, $\operatorname{ran}(\vec{v}\lceil\beta)=\bar{f}[\operatorname{ran}(\vec{u}\lceil\beta)]$ and $\mathbb{S}_{\beta}\left[\vec{v}\lceil\beta] \subseteq \mathbb{S}_{\beta}[\vec{u} \uparrow \beta]\right.$.

Consider $g \in \mathbb{V}^{\Sigma \mathbf{v}} \cap \mathbb{S}_{\beta}$. On the one hand, $\mathfrak{j}_{\beta ; y, B}(g)=\mathfrak{j}_{\alpha ; y, B}\left(\mathfrak{j}^{y}\left(\Omega_{T^{(1)}, \mathbf{v}}(g)\right)\right.$, where $\mathfrak{j}^{y}$ is the $\mathfrak{j}$ from the proof of Theorem 10.9, Case 2, for $y, v$ in place of $x, u$, and $\Omega_{T^{(1)}, \mathbf{v}}(g)\left(v^{\prime}\right)=g_{\left\langle v^{\prime}\right\rangle}$ for $v^{\prime} \in v$; then $\mathfrak{j}^{y}\left(\Omega_{T^{(1)}, \mathbf{v}}(g)\right)=g_{\left\langle v_{0}\right\rangle}$ and

$$
\left.\mathfrak{j}_{\beta ; y, B}(g)=\mathfrak{j}_{\alpha ; y, B}\left(g_{\left\langle v_{0}\right\rangle}\right)=g_{\left\langle v_{0}\right\rangle}\right\rangle\left(\left(\vec{v}\lceil\alpha)^{+}\right)=g(\vec{v}\lceil\alpha) .\right.
$$

On the other hand, $\varphi^{*}(g)\left(\left\langle u^{\prime}\right\rangle \frown t\right)=g\left(\varphi\left(\left\langle u^{\prime}\right\rangle \frown t\right)\right)=g\left(\left\langle\bar{f}\left(u^{\prime}\right)\right\rangle \frown \varphi^{u^{\prime}}(t)\right)=$ $g_{\left\langle\bar{f}\left(u^{\prime}\right)\right\rangle}\left(\varphi^{u^{\prime}}(t)\right)=\left(\varphi^{u^{\prime}}\right)^{*}\left(g_{\left\langle\bar{f}\left(u^{\prime}\right)\right\rangle}\right)(t) \quad u$-almost everywhere, so $\Omega_{T^{(1)}, \mathbf{u}}\left(\varphi^{*}(g)\right)\left(u^{\prime}\right)=$ $\left(\varphi^{u^{\prime}}\right)^{*}\left(g_{\left\langle\bar{f}\left(u^{\prime}\right)\right\rangle}\right) u$-almost everywhere, and then, by definition of $\mathfrak{j}=\mathfrak{j}^{x}$ and Transfer, $\mathfrak{j}^{x}\left(\Omega_{T^{1}, \mathbf{u}}\left(\varphi^{*}(g)\right)\right)=\left(\varphi^{u_{n}}\right)^{*}\left(g_{\left\langle\bar{f}\left(u_{n}\right)\right\rangle}\right)$. But $\bar{f}\left(u_{n}\right)=v_{0}$ and $\varphi^{u_{n}}$ is the naturally induced morphism of $u_{n}$ to $v_{0}$. Hence [note that $g_{\left\langle v_{0}\right\rangle} \in \mathbb{S}_{\alpha}$, so we can use the inductive assumption to justify the second equality]

$$
\mathfrak{j}_{\beta ; x, A}\left(\varphi^{*}(g)\right)=\mathfrak{j}_{\alpha ; x, A}\left(\left(\varphi^{u_{n}}\right)^{*}\left(g_{\left\langle v_{0}\right\rangle}\right)\right)=\mathfrak{j}_{\alpha ; y, B}\left(g_{\left\langle v_{0}\right\rangle}\right)=g_{\left\langle v_{0}\right\rangle}\left(\left(\vec{v}\lceil\alpha)^{+}\right)=g(\vec{v}\lceil\alpha) .\right.
$$

By comparing $(*)$ and $(* *)$ we get $\mathfrak{j}_{\beta ; y, B}(g)=\mathfrak{j}_{\beta ; x, A}\left(\varphi^{*}(g)\right)$, the commutativity of the diagram for $\beta$.

The theory SST$^{*}$ is used in this paper mainly as a technical tool. Our main interest is in two related theories that are introduced next.

We write $x \mathbf{M}_{\alpha} U$ as shorthand for:

"There exists a good $\alpha$-pedigree $\vec{u}=\left\langle u_{n}: n \leq \nu\right\rangle$ for $x$ over some $A \in \mathbb{S}_{\alpha}$ such that $U=u_{0} "$.

We note that $U$ is then an $\alpha$-type of $x$.

The theory $\mathbf{S S T}^{b}$ is SST plus $(\forall \alpha)\left(\mathbf{B}_{\alpha}\right)$, where

$$
\begin{gathered}
\left(\mathbf{B}_{\alpha}\right) \quad(\forall x, y)\left(\forall U, F \in \mathbb{S}_{\alpha}\right)\left[\left(\operatorname{ran} F \subseteq \operatorname{Dom} U \wedge x \mathbf{M}_{\alpha} U \wedge x=F(y)\right) \Rightarrow\right. \\
\left.\left(\exists V \in \mathbb{S}_{\alpha}\right)\left(U=\bar{F}(V) \wedge y \mathbf{M}_{\alpha} V\right)\right] .
\end{gathered}
$$


Later, we consider one more axiom:

$$
\begin{gathered}
\left(\mathbf{F}_{\alpha}\right) \quad(\forall x)\left(\forall U, V, F \in \mathbb{S}_{\alpha}\right)\left[\left(\operatorname{ran} F \subseteq \operatorname{Dom} U \wedge x \mathbf{M}_{\alpha} U \wedge U=\bar{F}(V)\right) \Rightarrow\right. \\
\left.(\exists y)\left(y \mathbf{M}_{\alpha} V \wedge x=F(y)\right)\right] .
\end{gathered}
$$

The theory SST $\mathbf{S T}^{b}+(\forall \alpha)\left(\mathbf{F}_{\alpha}\right)$ is denoted SST ${ }^{\sharp}$. It is equivalent to GRIST [see Section 12]. We refer to the conjunction of $(\forall \alpha)\left(\mathbf{B}_{\alpha}\right)$ and $(\forall \alpha)\left(\mathbf{F}_{\alpha}\right)$ as the Back and Forth Property.

Theorems 10.7, 10.9, and 10.10 establish the following.

\section{Corollary 10.11 SST $^{*} \vdash$ SST $^{b}$.}

In particular, every stratified ultrapower satisfies SST ${ }^{b}$ [Corollary 8.5].

Corollary 10.12 (ZFC) Every realization of $\mathbf{S S T}^{b, p}:=\mathbf{S S T}^{b}+$ Axiom of Primitivity is isomorphic to a stratified ultrapower of the universe.

In this sense, $\mathbf{S S T}^{b, p}$ [or $\mathbf{S S T}^{*, p}$ ] axiomatizes stratified ultrapowers.

Proof Working in SST $\mathbf{S}^{b, p}$, let $a \mathbf{M}_{0} U$, where $a \in A \in \mathbb{S}_{0}$, be such that $(\forall x)(\exists f \in$ $\left.\mathbb{S}_{0}\right)(x=f(a))$. By Theorem 10.9, $\mathfrak{j}_{0 ; a, A}$ is an isomorphism between $[\mathcal{U} \ell t(\mathbb{V} ; \Sigma \mathbf{U})]^{\mathbb{S}_{0}}$ and $(\mathbb{V},=, \in, \sqsubseteq)$ [because $\left.\mathbb{S}_{0}\left[\vec{u}^{+}\right]=\mathbb{S}_{0}[a]=\mathbb{V}\right]$.

Working now in $\mathbf{Z F C}$; if $\mathfrak{M}$ is a realistic interpretation of $\mathbf{S S T}^{b, p}$, the previous statement holds in $\mathfrak{M}$, and there is an isomorphism of interpretations $\left[[\mathcal{U} \ell t(\mathbb{V} ; \Sigma \mathfrak{k}(\mathbf{U}))]^{\mathbb{S}_{0}}\right]^{\mathfrak{M}}$ and $[(\mathbb{V},=, \in, \sqsubseteq)]^{\mathfrak{M}}=\mathfrak{M}$, for a suitable $U$. As $\mathbb{S}_{0}{ }^{\mathfrak{M}}$ is isomorphic to $\mathbb{V}, \mathfrak{M}$ is isomorphic to $\mathcal{U} \ell t(\mathbb{V} ; \Sigma \mathbf{U})$.

Definition 10.13 $\left(\mathbf{S S T}^{b}\right) \mathbb{S}_{\alpha}[[x]]:=\mathbb{S}_{\alpha}[\vec{u}]$, where $\vec{u}$ is some good $\alpha$-pedigree for $x$.

If $\vec{u}$ and $\vec{v}$ are $\alpha$-pedigrees for $x$, then $\mathbb{S}_{\alpha}[\vec{u}]=\mathbb{S}_{\alpha}[\vec{v}]$ by Proposition 10.4 , so this class is uniquely determined by $x$. From the isomorphism property $(\mathfrak{j})$ and Corollary 8.5 it follows that $\left(\mathbb{S}_{\alpha}[[x]],=, \in, \sqsubseteq_{\alpha}\right) \vDash$ SST $^{*}$.

As an immediate consequence we have:

Inner Standardization into $\mathbb{S}_{\alpha}$ is a consequence of the remaining axioms of $\mathbf{S S T}^{b}$.

Proposition 10.14 Proposition 10.5, Corollary 10.6 and Theorems 10.7, 10.9 and 10.10 hold in $\mathbf{S S T}^{b}$. 
Proof Theorems 10.7 and 10.9 are immediate consequences of $\mathbf{B}_{\alpha}$.

Proposition 10.5: Let $\vec{u}=\left\langle u_{0}, \ldots, u_{\nu}\right\rangle$ be an $\alpha$-pedigree for $x$ and $\alpha \sqsubseteq \beta$. Trivially, $\mathbb{S}_{\alpha}[[\langle\vec{u}, \beta\rangle]] \vDash " \vec{u}$ is a pedigree for $x$ ". As $\mathbb{S}_{\alpha}[[\langle\vec{u}, \beta\rangle]] \vDash$ SST $^{*}$, Proposition 10.5 holds in $\mathbb{S}_{\alpha}[[\langle\vec{u}, \beta\rangle]]$, yielding $n \in \omega$ such that $u_{n} \sqsubseteq \beta \sqsubset u_{n+1}$ [or $n=\nu$ and $u_{\nu} \sqsubseteq \beta$ ]. Then $\vec{u} \uparrow \beta$ is defined, and it is a $\beta$-pedigree for $x$.

Theorem 10.10: Given $\alpha$-pedigrees $\vec{u}$ and $\vec{v}$ for $x$ and $y$, resp., and $f$ as in Theorem 10.10 , consider $\mathbb{S}_{\alpha}[[\langle\vec{u}, \vec{v}\rangle]]$. Note that $\vec{u}$ and $\vec{v}$ are $\alpha$-pedigrees in $\mathbb{S}_{\alpha}[[\langle\vec{u}, \vec{v}\rangle]]$, and Theorem 10.10 holds in $\mathbb{S}_{\alpha}[[\langle\vec{u}, \vec{v}\rangle]]$, yielding $\varphi \in \mathbb{S}_{\alpha}$ and a diagram that has the required properties in $\mathbb{S}_{\alpha}[[\langle\vec{u}, \vec{v}\rangle]]$. It is easy to check that these properties remain true in $\mathbb{V}$.

\section{Proposition 10.15 (ZFC)}

If $\varphi: \mathcal{U}^{2} \rightarrow \mathcal{U}^{1}$ is a morphism and $\mathcal{U} \ell t\left(\mathbb{V} ; \mathcal{U}^{1}\right) \vDash$ " $\vec{u}$ is a pedigree for $f$ over $\mathfrak{k}_{\Sigma \mathcal{U}^{1}}(A)$ ", then $\mathcal{U} \ell t\left(\mathbb{V} ; \mathcal{U}^{2}\right) \vDash " \varphi^{*}(\vec{u})$ is a pedigree for $\varphi^{*}(f)$ over $\mathfrak{k}_{\Sigma \mathcal{U}^{2}}(A)$ ".

Proof $\varphi^{*}: \mathcal{U} \ell t\left(\mathbb{V} ; \mathcal{U}^{1}\right) \rightarrow \mathcal{U} \ell t\left(\mathbb{V} ; \mathcal{U}^{2}\right)$ is an $\in$-elementary embedding, preserves $\sqsubseteq$, and $\varphi^{*}\left(\mathfrak{k}_{\Sigma \mathcal{U}^{1}}(A)\right)=\mathfrak{k}_{\Sigma \mathcal{U}^{2}}(A)$ for all $A \in \mathbb{V}$. [In this proof, we identify $\mathfrak{k}(A)$ and $A$ when there is no danger of misunderstanding.] It follows immediately that $\varphi^{*}(\vec{u})$ satisfies the conditions (i) and (ii) from Definition 10.1. We prove (iii) and (iv) by induction on $\operatorname{rank} T^{2}\left[T^{2}:=T_{\mathcal{U}^{2}} ; T^{1}:=T_{\mathcal{U}^{1}}\right]$.

As usual, everything is trivial when $\operatorname{rank} T^{2}=0$, so we assume $T^{2}=\{0\} \cup \bigcup_{j \in J}\langle j\rangle \frown$ $T_{j}$. We let $D^{1}:=D_{T^{\prime}}$ for $T^{\prime}:=T_{\mathcal{U}^{1}}^{(1)}$, and $D^{2}:=D_{T^{\prime \prime}}$ for $T^{\prime \prime}:=T_{\mathcal{U}^{2}}^{(1)}$.

By Łoś Theorem, $\vec{u} \in \mathbb{V}^{\Sigma \mathcal{U}^{1}}$ is a function whose values $\Sigma \mathcal{U}^{1}$-almost everywhere are finite sequences of stratified ultrafilters over $A$, that is, without loss of generality for all $t \in \Sigma T^{1}, \vec{u}(t)=\left\langle u_{0}(t), \ldots u_{\nu(t)}(t)\right\rangle$ where $\nu(t) \in \omega$. Then $\varphi^{*}(\vec{u}) \in \mathbb{V}^{\Sigma \mathcal{U}^{2}}$ is the function defined for all $s \in \Sigma \operatorname{dom} \varphi$ by $\varphi^{*}(\vec{u})(s)=\left\langle u_{0}(t), \ldots u_{\nu(t)}(t)\right\rangle$, for $t=\varphi(s)$. Let $\lambda \in \mathbb{V}^{\Sigma \mathcal{U}^{2}}$ be such that $\mathcal{U} \ell t\left(\mathbb{V} ; \mathcal{U}^{2}\right) \vDash \lambda \leq \varphi^{*}(\nu)$; ie, without loss of generality for all $s \in \Sigma \operatorname{dom} \varphi, \lambda(s) \leq \nu(\varphi(s))$. We note that $\mathcal{U} \ell t\left(\mathbb{V} ; \mathcal{U}^{2}\right) \vDash \varphi^{*}(\vec{u})_{\mathfrak{E}_{\Sigma \mathcal{U}^{2}}(n)}=\varphi^{*}\left(u_{n}\right)$, for all $n \in \omega$ [where $\left.u_{n}: t \mapsto u_{n}(t)\right]$. There are two cases to consider.

\section{Case (c).}

$\varphi(\langle j\rangle)=0$ and $\varphi_{\langle j\rangle}: \mathcal{U}_{\langle j\rangle}^{2} \rightarrow \mathcal{U}^{1}$, for all $j \in[\operatorname{dom} \varphi]_{0} \in \mathcal{U}^{2}(0)$. We note that $\varphi^{*}(h)_{\langle j\rangle}=\varphi_{\langle j\rangle}^{*}(h)$ for any $h \in \mathbb{V}^{\Sigma \mathcal{U}^{1}}$, in this case.

By the inductive assumption, $\varphi_{\langle j\rangle}^{*}(\vec{u})$ is a pedigree for $\varphi_{\langle j\rangle}^{*}(f)$ over $A$ in $\mathcal{U} \ell t\left(\mathbb{V} ; \mathcal{U}_{\langle j\rangle}^{2}\right)$. By the Factoring Theorem, $\left(\mathcal{U} \ell\left(\mathbb{V} ; \Sigma \mathcal{U}^{2}\right), \sqsubseteq \mathcal{U}^{2} ; D^{2}\right) \vDash " \varphi^{*}(\vec{u})$ is a pedigree for $\varphi^{*}(f)$ 
over $A$ ”. But $u_{0}==_{\Sigma \mathcal{U}^{1}} \mathfrak{k}_{\Sigma \mathcal{U}^{1}}(w)$ for some $w \in \mathbb{V}$, so $\varphi^{*}\left(u_{0}\right)=_{\Sigma \mathcal{U}^{2}} \mathfrak{k}_{\Sigma \mathcal{U}^{2}}(w) \in$ $\mathbb{S}_{0}^{\mathcal{U} \ell t\left(\mathbb{V} ; \mathcal{U}^{2}\right)}$; this means that $\varphi^{*}(\vec{u})$ is also a pedigree for $\varphi^{*}(f)$ over $A$ in $\mathcal{U} \ell t\left(\mathbb{V} ; \Sigma \mathcal{U}^{2}\right)=$ $\left(\mathcal{U} \ell\left(\mathbb{V} ; \Sigma \mathcal{U}^{2}\right), \sqsubseteq_{\mathcal{U}^{2}}\right)$.

\section{Case (p).}

$[\varphi]_{0}:\left[T^{2}\right]_{0} \rightarrow\left[T^{1}\right]_{0}, \mathcal{U}^{1}(0)=[\varphi]_{0}\left[\mathcal{U}^{2}(0)\right]$ and $\varphi_{\langle j\rangle}: \mathcal{U}_{\langle j\rangle}^{2} \rightarrow \mathcal{U}_{\varphi(\langle j\rangle)}^{1}$ for all $j \in$ $[\operatorname{dom} \varphi]_{0} \in \mathcal{U}^{2}(0)$. We note that $\varphi_{\langle j\rangle}^{*}\left(h_{\varphi(\langle j\rangle)}\right)=\varphi^{*}(h)_{\langle j\rangle}$ for any $h \in \mathbb{V}^{\Sigma \mathcal{U}^{1}}$, in this case. We distinguish two subcases.

Subcase (i): $\mathcal{U} \ell t\left(\mathbb{V} ; \mathcal{U}^{1}\right) \vDash D^{1} \sqsubset u_{1}$.

Then also $\left(\mathcal{U} \ell\left(\mathbb{V} ; \Sigma \mathcal{U}^{1}\right), \sqsubseteq_{\mathcal{U}^{1} ; D^{1}}\right) \vDash " \vec{u}$ is a pedigree for $f$ over $A$ ". By the Factoring Theorem, $\mathcal{U} \ell t\left(\mathbb{V} ; \mathcal{U}_{\langle i\rangle}^{1}\right) \vDash " \vec{u}_{\langle i\rangle}$ is a pedigree for $f_{\langle i\rangle}$ over $A$ " for $\mathcal{U}^{1}(0)$-almost all $i$. So, by the inductive assumption, $\mathcal{U} \ell t\left(\mathbb{V} ; \mathcal{U}_{\langle j\rangle}^{2}\right) \vDash " \varphi_{\langle j\rangle}^{*}\left(\vec{u}_{\varphi(\langle j\rangle)}\right)$ is a pedigree for $\varphi_{\langle j\rangle}^{*}\left(f_{\varphi(\langle j\rangle)}\right)$ over $A^{\prime}$ and $\mathcal{U} \ell t\left(\mathbb{V} ; \mathcal{U}_{\langle j\rangle}^{2}\right) \vDash " \varphi^{*}(\vec{u})_{\langle j\rangle}$ is a pedigree for $\varphi^{*}(f)_{\langle j\rangle}$ over $A$ ", for $\mathcal{U}^{2}(0)$-almost all $j$. The Factoring Theorem leads to the conclusion that $\left(\mathcal{U} \ell\left(\mathbb{V} ; \Sigma \mathcal{U}^{2}\right), \sqsubseteq_{\mathcal{U}^{2} ; D^{2}} \vDash{ }^{*} \varphi^{*}(\vec{u})\right.$ is a pedigree for $\varphi^{*}(f)$ over $A$ ". As in case (c) now, $\varphi^{*}\left(u_{0}\right)=\mathcal{U}^{2} \mathfrak{k}_{\Sigma \mathcal{U}^{2}}(w)$ for some $w \in \mathbb{V}$, so $\varphi^{*}\left(u_{0}\right) \in \mathbb{S}_{0}^{\mathcal{U} \ell t\left(\mathbb{V} ; \mathcal{U}^{2}\right)}$, and we have also $\mathcal{U} \ell t\left(\mathbb{V} ; \Sigma \mathcal{U}^{2}\right) \vDash " \varphi^{*}(\vec{u})$ is a pedigree for $\varphi^{*}(f)$ over $A$ ".

Subcase (ii): $\mathcal{U} \ell t\left(\mathbb{V} ; \mathcal{U}^{1}\right) \vDash D^{1} \boxminus u_{1}$.

Let $\vec{u}^{+}$denote a function defined by $\vec{u}^{+}(t):=\left\langle u_{1}(t), \ldots u_{\nu(t)}(t)\right\rangle$ for $\Sigma \mathcal{U}^{1}$-almost all $t \in \Sigma T^{1}$. The notational ambiguity is harmless, as $\mathcal{U} \ell t\left(\mathbb{V} ; \Sigma \mathcal{U}^{1}\right) \vDash \underline{u^{+}}=\vec{u}^{+}$. Then $\left(\mathcal{U} \ell\left(\mathbb{V} ; \Sigma \mathcal{U}^{1}\right), \sqsubseteq_{\mathcal{U}^{1} ; D^{1}}\right) \vDash$ “ $\vec{u}^{+}$is a pedigree for $f$ over $A$ ".

By the Factoring Theorem, $\mathcal{U} \ell t\left(\mathbb{V} ; \mathcal{U}_{\langle i\rangle}^{1}\right) \vDash " \vec{u}_{\langle i\rangle}^{+}$is a pedigree for $f_{\langle i\rangle}$ over $A$ " for $\mathcal{U}^{1}(0)$-almost all $i$. The inductive assumption gives that $\mathcal{U} \ell t\left(\mathbb{V} ; \mathcal{U}_{\langle j\rangle}^{2}\right) \vDash " \varphi^{*}\left(\vec{u}^{+}\right)_{\langle j\rangle}$ is a pedigree for $\varphi^{*}(f)_{\langle j\rangle}$ over $A^{\text {" for }} \mathcal{U}^{2}(0)$-almost all $j$.

By the Factoring Theorem one more time, $\left(\mathcal{U} \ell\left(\mathbb{V} ; \Sigma \mathcal{U}^{2}\right), \sqsubseteq \mathcal{U}^{2} ; D^{2}\right) \vDash " \varphi^{*}\left(\vec{u}^{+}\right)$is a pedigree for $\varphi^{*}(f)$ over $A$ "; also

$\left(\mathcal{U} \ell\left(\mathbb{V} ; \Sigma \mathcal{U}^{2}\right), \sqsubseteq_{\mathcal{U}^{2}}\right) \vDash “ \varphi^{*}(\vec{u})=\left\langle\varphi^{*}\left(u_{0}\right)\right\rangle \frown \varphi^{*}\left(\vec{u}^{+}\right) \wedge \varphi^{*}\left(u_{0}\right) \sqsubseteq 0 \wedge \varphi^{*}\left(u_{1}\right) \boxminus D^{2} "$.

In order to prove that $\varphi^{*}(\vec{u})$ is a pedigree for $\varphi^{*}(f)$ over $A$ in $\mathcal{U} \ell t\left(\mathbb{V} ; \Sigma \mathcal{U}^{2}\right)$, it remains to show that $\mathcal{U} \ell t\left(\mathbb{V} ; \Sigma \mathcal{U}^{2}\right) \vDash$ "k $_{\Sigma \mathcal{U}^{2}}(X) \in \varphi^{*}\left(u_{0}\right) \Rightarrow \varphi^{*}\left(u_{1}\right) \in \mathfrak{k}_{\Sigma \mathcal{U}^{2}}(X)$ ", for all $X \in \mathbb{V}$. But $u_{0}=_{\Sigma \mathcal{U}^{1}} \mathfrak{k}_{\Sigma \mathcal{U}^{1}}(w)$ for some $w \in \boldsymbol{\beta}_{\infty} A$, hence $\varphi^{*}\left(u_{0}\right)=_{\Sigma \mathcal{U}^{2}} \mathfrak{k}_{\Sigma \mathcal{U}^{2}}(w)$ and the antecedent implies that $X \in w$. It follows that $\mathcal{U} \ell t\left(\mathbb{V} ; \mathcal{U}^{1}\right) \vDash u_{1} \in \mathfrak{k}_{\Sigma \mathcal{U}^{1}}(X)$ and finally $\mathcal{U} \ell t\left(\mathbb{V} ; \mathcal{U}^{2}\right) \vDash \varphi^{*}\left(u_{1}\right) \in \mathfrak{k}_{\Sigma \mathcal{U}^{2}}(X)$. 
Proposition 10.16 (ZFC) If $\varphi: \mathcal{U}^{2} \rightarrow \mathcal{U}^{1}$ is a morphism and $\mathcal{U} \ell t\left(\mathbb{V} ; \mathcal{U}{ }^{1}\right) \vDash " 0 \sqsubset$ $h \wedge\left(\forall x \in \mathfrak{k}_{\Sigma \mathcal{U}^{1}}(A)\right)(x \sqsubset h \Rightarrow\langle x, y\rangle \in R)$, then $\mathcal{U} \ell t\left(\mathbb{V} ; \mathcal{U}^{2}\right) \vDash\left(\forall x \in \mathfrak{k}_{\Sigma \mathcal{U}^{2}}(A)\right)[x \sqsubset$ $\left.\varphi^{*}(h) \Rightarrow\left\langle x, \varphi^{*}(y)\right\rangle \in \varphi^{*}(R)\right]$.

Proof We proceed by induction on the rank of $\mathcal{U}^{2}$.

\section{Case (c).}

By the inductive assumption, we have $\mathcal{U} \ell t\left(\mathbb{V} ; \mathcal{U}_{\langle j\rangle}^{2}\right) \vDash(\forall x \in \mathfrak{k}(A))\left(x \sqsubset \varphi_{\langle j\rangle}^{*}(h) \Rightarrow\right.$ $\left.\left\langle x, \varphi_{\langle j\rangle}^{*}(y)\right\rangle \in \varphi_{\langle j\rangle}^{*}(R)\right)$ for each $j \in[\operatorname{dom} \varphi]_{0} \in \mathcal{U}^{2}(0)$. Hence, by the Factoring Theorem, $\left(\mathcal{U} \ell\left(\mathbb{V} ; \Sigma \mathcal{U}^{2}\right), \sqsubseteq_{\mathcal{U}^{2} ; D^{2}}\right) \vDash " 0 \sqsubset \varphi^{*}(h) \wedge(\forall x \in \mathfrak{k}(A))\left[\left(x \sqsubset \varphi^{*}(h) \Rightarrow\left\langle x, \varphi^{*}(y)\right\rangle \in\right.\right.$ $\left.\left.\varphi^{*}(R)\right)\right]$ ". As $D^{2} \sqsubset_{\mathcal{U}^{2}} \varphi^{*}(h)$ implies $x \sqsubset_{\mathcal{U}^{2} ; D^{2}} \varphi^{*}(h) \Leftrightarrow x \sqsubset_{\mathcal{U}^{2}} \varphi^{*}(h)$, we have $\mathcal{U} \ell t\left(\mathbb{V} ; \mathcal{U}^{2}\right) \vDash(\forall x \in \mathfrak{k}(A))\left[x \sqsubset \varphi^{*}(h) \Rightarrow\left\langle x, \varphi^{*}(y)\right\rangle \in \varphi^{*}(R)\right]$.

\section{Case (p).}

Subcase (i): $\mathcal{U} \ell t\left(\mathbb{V} ; \mathcal{U}^{1}\right) \vDash D^{1} \sqsubset h$.

Then $\left(\mathcal{U} \ell\left(\mathbb{V} ; \Sigma \mathcal{U}^{1}\right), \sqsubseteq \mathcal{U}^{1} ; D^{1}\right) \vDash " 0 \sqsubset h \wedge(\forall x \in \mathfrak{k}(A))(x \sqsubset h \Rightarrow\langle x, y\rangle \in R)$ ", and by the Factoring Theorem, $\mathcal{U} \ell t\left(\mathbb{V} ; \mathcal{U}_{\langle i\rangle}^{1}\right) \vDash " 0 \sqsubset \underline{h_{\langle i\rangle}} \wedge(\forall x \in \mathfrak{k}(A))\left(x \sqsubset \underline{h_{\langle i\rangle}} \Rightarrow\left\langle x, \underline{\left.y_{\langle i\rangle}\right\rangle} \in\right.\right.$

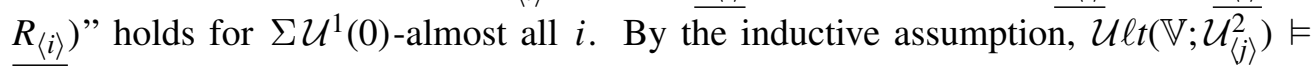
$\overline{{ }^{\prime} 0} \sqsubset \varphi^{*}(h)_{\langle j\rangle} \wedge(\forall x \in \mathfrak{k}(A))\left[x \sqsubset \varphi^{*}(h)_{\langle j\rangle} \Rightarrow\left\langle x, \varphi^{*}(y)_{\langle j\rangle}\right\rangle \in \varphi^{*}(R)_{\langle j\rangle}\right]$ ", and the conclusion follows as in case (c).

Subcase (ii): $\mathcal{U} \ell t\left(\mathbb{V} ; \mathcal{U}^{1}\right) \vDash D^{1} \boxminus h$.

Then $\varphi^{*}(h) \boxminus_{\mathcal{U}^{2}} \varphi^{*}\left(D^{1}\right) \boxminus_{\mathcal{U}^{2}} D^{2}$, and $\mathcal{U} \ell t\left(\mathbb{V} ; \mathcal{U}^{2}\right) \vDash “ x \in \mathfrak{k}(A) \wedge x \sqsubset \varphi^{*}(h)$ ” implies that $\mathcal{U} \ell t\left(\mathbb{V} ; \mathcal{U}^{2}\right) \vDash “ x \in \mathfrak{k}(A) \wedge x \sqsubseteq 0$ ", ie, $x=\mathfrak{k}(X)$ for some $X \in A$. Since $\mathcal{U} \ell t\left(\mathbb{V} ; \mathcal{U}^{1}\right) \vDash\langle\mathfrak{k}(X), y\rangle \in R$ for all such $X, \mathcal{U} \ell t\left(\mathbb{V} ; \mathcal{U}^{2}\right) \vDash\left\langle\mathfrak{k}(X), \varphi^{*}(y)\right\rangle \in \varphi^{*}(R)$ for all such $x$.

Proposition $10.17\left(\mathbf{S S T}^{b}\right)$ Let $A \in \mathbb{S}_{\alpha}, x \in A$ and $x, \vec{u} \in \mathbb{S}_{\alpha}[[y]]$.

If $\left(\mathbb{S}_{\alpha}[[y]],=, \in, \sqsubseteq_{\alpha}\right) \vDash$ " $\vec{u}$ is the pedigree for $x$ over $A$ ", then $\vec{u}$ is the $\alpha$-pedigree for $x$ over $A$.

Proof Transfer $\left[\mathbb{S}_{\alpha}[[y]] \preccurlyeq \mathbb{V}\right]$ immediately implies that $\vec{u}=\left\langle u_{n}: n \leq \nu\right\rangle$ where $\nu \in \omega \cap \mathbb{S}_{\alpha}[[y]]$, every $u_{n} \in \boldsymbol{\beta}_{\infty} A$, and $u_{0} \in \mathbb{S}_{\alpha}, u_{\nu}=x$. We have to prove that (iii) and (iv) from Definition 10.1 are also satisfied.

If (iii) fails, then there are $n<m \leq \nu$ such that $u_{m} \sqsubseteq_{\alpha} u_{n}$. If (iv) fails, then there is $n<\nu$ and $X \in u_{n}$ such that $X \sqsubset_{\alpha} u_{n+1}$ and $u_{n+1} \notin X$. Let $z=\langle y,\langle n, m\rangle\rangle$ in the first case and $z=\langle y,\langle n, X\rangle\rangle$ in the second case. 
Let $\vec{v}^{1}$ and $\vec{v}^{2}$ be $\alpha$-pedigrees for $y$ over $B$ and $z$ over $C$ resp., where $B, C \in \mathbb{S}_{\alpha}$, and $\pi_{1}: C \rightarrow B$. Then $y=\pi_{1}(z)$ and Proposition 10.14 [Theorem 10.10] gives a commuting diagram $\left[\varphi\right.$ is induced by $\left.\overline{\pi_{1}}\right]$.

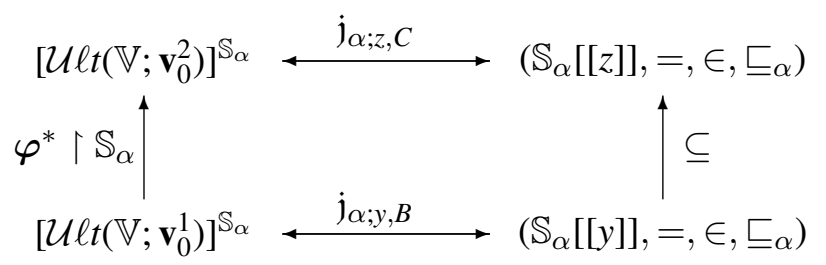

As $n, m \in \mathbb{S}_{\alpha}[[z]]$ in the first case, and $n, X \in \mathbb{S}_{\alpha}[[z]]$ in the second case, (iii) or (iv) fails in $\mathbb{S}_{\alpha}[[z]]$, so $\mathbb{S}_{\alpha}[[z]] \not \models " \vec{u}$ is the pedigree for $x$ over $A$ ".

Let $\mathcal{S}:=\mathfrak{j}_{\alpha ; y, B}^{-1}(\vec{u}), \quad \mathcal{X}:=\mathfrak{j}_{\alpha ; y, B}^{-1}(x), \mathcal{A}:=\mathfrak{j}_{\alpha ; y, B}^{-1}(A)$.

$\mathbb{S}_{\alpha} \vDash \mathbf{Z F C}$, so Proposition 10.15 holds in $\mathbb{S}_{\alpha}$. But, in $\mathbb{S}_{\alpha}, \mathcal{U} \ell t\left(\mathbb{V} ; \mathbf{v}_{0}^{1}\right) \vDash$ " $\mathcal{S}$ is a pedigree for $\mathcal{X}$ over $\mathcal{A}$ ", therefore $\mathcal{U} \ell t\left(\mathbb{V} ; \mathbf{v}_{0}^{2}\right) \vDash " \varphi^{*}(\mathcal{S})$ is a pedigree for $\varphi^{*}(\mathcal{X})$ over $\varphi^{*}(\mathcal{A}) ", \mathrm{j}_{\alpha ; z, C}\left(\varphi^{*}(\mathcal{S})\right)=\vec{u}, \mathrm{j}_{\alpha ; z, C}\left(\varphi^{*}(\mathcal{X})\right)=x, \mathrm{j}_{\alpha ; z, C}\left(\varphi^{*}(\mathcal{A})\right)=A$, and so $\left(\mathbb{S}_{\alpha}[[z]],=, \in, \sqsubseteq_{\alpha}\right) \vDash " \vec{u}$ is the pedigree for $x$ over $A$ ", a contradiction.

Corollary 10.18 (Closure under pedigrees)

If $x \in A \in \mathbb{S}_{\alpha}$ and $x \in \mathbb{S}_{\alpha}[[y]]$, then the $\alpha$-pedigree for $x$ over $A$ belongs to $\mathbb{S}_{\alpha}[[y]]$.

Proof $\left(\mathbb{S}_{\alpha}[[y]],=, \in, \sqsubseteq_{\alpha}\right) \vDash$ SST $^{*}$, so $\left(\mathbb{S}_{\alpha}[[y]],=, \in, \sqsubseteq_{\alpha}\right) \vDash "(\exists ! \vec{u})(\vec{u}$ is a pedigree for $x$ over $A$ ". By Proposition 10.17, this $\vec{u} \in \mathbb{S}_{\alpha}[[y]]$ is the $\alpha$-pedigree for $x$ over A.

Corollary 10.19 $\mathbb{S}_{\alpha}[[y]]$ is the smallest $\mathbb{U} \preccurlyeq \mathbb{V}$ such that $\mathbb{S}_{\alpha} \subseteq \mathbb{U}, y \in \mathbb{U}$, and with each $x \in \mathbb{U}$ also an $\alpha$-pedigree for $x$ is in $\mathbb{U}$.

Proposition 10.20 (ZFC) Let $U \in \boldsymbol{\beta}_{\infty} A$ and let $\mathbf{U}$ be its associated TOU.

Define $D:=D_{\mathbf{U}}:=D_{T_{\mathbf{U}}}, E:=E_{\mathbf{U}}:=E_{T_{\mathbf{U}}} \in \mathbb{V}^{\Sigma T_{\mathbf{U}}}$ by $D_{\mathbf{U}}(t)=t$ and $E_{\mathbf{U}}(t)=t_{n}$, where $t=\left\langle t_{0}, \ldots, t_{n}\right\rangle \in \Sigma T_{\mathbf{U}} ; E_{\mathbf{U}}(t)=U$ if $t=0$ (ie, $U \in \boldsymbol{\beta}_{0} A$ ) [see Corollary 7.3]. Then $\mathcal{U} \ell t(\mathbb{V} ; \mathbf{U}) \vDash "\left\langle\mathfrak{k}_{\Sigma} \mathbf{U}(U)\right\rangle \frown D$ is the pedigree for $E$ over $\mathfrak{k}_{\Sigma} \mathbf{U}(A)$ ".

Moreover, $\mathcal{U} \ell t(\mathbb{V} ; \mathbf{U}) \vDash(\forall f \sqsupset 0)(\exists \mu \in \omega)\left(f \boxminus D_{\mu}\right)$.

Proof We write $\mathfrak{k}$ for $\mathfrak{k}_{\Sigma \mathbf{U}}$ throughout. The case when $U \in \boldsymbol{\beta}_{0} A$ is trivial. Otherwise, by Definition 9.8, for all $t \in \Sigma T_{\mathbf{U}}, t_{n} \in \boldsymbol{\beta}_{0} A=A$; it follows that $\mathcal{U} \ell t(\mathbb{V} ; \mathbf{U}) \vDash$ $E \in \mathfrak{k}(A)$. From this definition it is equally clear that $\mathcal{U} \ell t(\mathbb{V} ; \mathbf{U}) \vDash \cdot\langle\mathfrak{k}(U)\rangle \frown D$ is 
a sequence of stratified ultrafilters with domain $=\nu+2 \in \omega$ and $D_{\nu}=e$ ", where $\nu(t)=n$ for all $t=\left\langle t_{0}, \ldots, t_{n}\right\rangle \in \Sigma T_{\mathbf{U}}$.

We prove the clauses (iii) and (iv) in the definition of pedigrees.

If $\mathcal{U} \ell t(\mathbb{V} ; \mathbf{U}) \vDash \mu \leq \nu$, then $\mu(t) \leq \nu(t)=n$ for $\Sigma \mathbf{U}$-almost all $t \in \Sigma T_{\mathbf{U}}$, and we define $D_{\mu}$ by $D_{\mu}(t):=t_{\mu(t)}$. The notational ambiguity is again harmless, as $\mathcal{U} \ell t(\mathbb{V} ; \mathbf{U}) \vDash D_{\mu}=D_{\mu}$. We note that $T\left(D_{\mu}\right) \equiv_{\Sigma \mathbf{U}} T_{\mu}^{\prime}:=\left\{t\left\lceil m: m \leq \mu(t) \wedge t \in \Sigma T_{\mathcal{U}}\right\}\right.$ [Corollary 7.3(c)], and $\lambda_{T\left(D_{\mu}\right)}(t)=\mu(t)+1$ for $\Sigma \mathbf{U}$-almost all $t$ [Definition 6.9]. Hence $\mathcal{U} \ell t(\mathbb{V} ; \mathbf{U}) \vDash “ \mu_{1}<\mu_{2} \leq \nu \Rightarrow D_{\mu_{1}} \sqsubset D_{\mu_{2}}$ ”. As $T^{(0)}=\{0\} \prec_{\mathbf{U}} T\left(D_{\mathfrak{k}(0)}\right)$, we have also $\mathcal{U} \ell t(\mathbb{V} ; \mathbf{U}) \vDash \mathfrak{k}(U) \sqsubset D_{0}$. These results establish (iii).

Also, for every $f \in \mathbb{V}^{\Sigma \mathbf{U}}$ with $T^{(0)} \prec \mathbf{U} T(f)$ there is $\mu \leq_{\Sigma \mathbf{U}} \nu$ such that $\lambda_{T(f)}(t)=$ $\mu(t)+1$ for $\Sigma \mathbf{U}$-almost all $t$. Then $\lambda_{T(f)} \equiv_{\mathbf{U}} \lambda_{T\left(D_{\mu}\right)}$ and $\mathcal{U} \ell t(\mathbb{V} ; \mathbf{U}) \vDash f \boxminus D_{\mu}$.

It remains to prove (iv).

If $\mathcal{U} \ell t(\mathbb{V} ; \mathbf{U}) \vDash " g \in \mathfrak{k}(U) \wedge g \sqsubset D_{0}$ ", then $g=\mathfrak{k}(S)$ for some $S \in U$, and for all $\langle v\rangle \frown t^{\prime} \in \Sigma T_{\mathbf{U}}$ where $v \in S$, we get $D_{\mathfrak{k}(0)}\left(\langle v\rangle \frown t^{\prime}\right)=v \in S=g\left(\langle v\rangle \frown t^{\prime}\right)$, ie, $\mathcal{U} \ell t(\mathbb{V} ; \mathbf{U}) \vDash D_{0} \in g$.

If $\mathcal{U} \ell t(\mathbb{V} ; \mathbf{U}) \vDash$ “ $g \in D_{\mu} \wedge \mu^{\prime}=\mu+1 \wedge g \sqsubset D_{\mu^{\prime}}$ ", then $T(g) \preccurlyeq \Sigma \mathbf{U} T\left(D_{\mu}\right)$ and there is $\bar{g} \in \mathbb{V}^{\Sigma T\left(D_{\mu}\right)}$ such that, for $\Sigma \mathbf{U}$-almost all $t, g(t)=\bar{g}(s) \in D_{\mu}(t)$ where $s=t \uparrow(\mu(t)+1)=\left\langle t_{0}, \ldots, t_{\mu(t)}\right\rangle$. Then for all $v \in \bar{g}(s)$ and all appropriate $t^{\prime}$, $D_{\mu^{\prime}}\left(s \frown\langle v\rangle \frown t^{\prime}\right)=v \in \bar{g}(s)=g\left(s \frown\langle v\rangle \frown t^{\prime}\right)$. We see that for $\Sigma \mathbf{U}$-almost all $t$, $D_{\mu^{\prime}}(t) \in g(t)$, ie, $\mathcal{U} \ell t(\mathbb{V} ; \mathbf{U}) \vDash D_{\mu+1} \in g$. This completes the verification of (iv).

Proposition 10.21 (ZFC) Let $\mathcal{U} \ell t(\mathbb{V} ; \mathcal{U}) \vDash " \vec{u}$ is the pedigree for $f$ over $\mathfrak{k}_{\Sigma \mathcal{U}}(A) \wedge$ $u_{0}=\mathfrak{k}_{\Sigma \mathcal{U}}(U)$ ". There is a morphism $\varphi: \mathcal{U} \rightarrow \mathbf{U}$ such that $\mathcal{U} \ell t(\mathbb{V} ; \mathcal{U}) \vDash \varphi \uparrow \Sigma T_{\mathcal{U}}=$ $\vec{u}^{+}$. In particular, $\mathcal{U} \ell t(\mathbb{V} ; \mathcal{U}) \vDash " \varphi^{*}\left(D_{\mathbf{U}}\right)=\vec{u}^{+} "$ and $\mathcal{U} \ell t(\mathbb{V} ; \mathcal{U}) \vDash " \varphi^{*}\left(E_{\mathbf{U}}\right)=f "$.

In this precise sense, "the pedigree for $f$ is a morphism onto the TOU associated to the type of $f$ ". We note that $\varphi$ is uniquely determined: if $\varphi^{\prime}$ is another morphism as in 10.21, then $\varphi^{\prime} \uparrow \Sigma T_{\mathcal{U}}=\Sigma \mathcal{U} \varphi \uparrow \Sigma T_{\mathcal{U}}$.

Proof We proceed by induction on $\operatorname{rank} T_{\mathcal{U}}$.

If $\operatorname{rank} T_{\mathcal{U}}=0$, then $\mathcal{U}=0, f=\Sigma \mathcal{U} \mathfrak{k}_{\Sigma \mathcal{U}}(a)$ for some $a \in A, U=a, \mathbf{U}=0$, and $\varphi$ is the trivial morphism $\varphi(0)=0$. So $\mathcal{U} \ell t(\mathbb{V} ; \mathcal{U}) \vDash " \varphi \mid \Sigma T_{\mathcal{U}}=\vec{u}^{+}$" is true.

Let $T_{\mathcal{U}}=\{0\} \cup \bigcup_{i \in I}\langle i\rangle \frown T_{i}$. We assume without loss of generality that $\operatorname{ran} f \subseteq A$. By the inductive assumption, for each $i \in I$ let $\mathcal{U} \ell t\left(\mathbb{V} ; \mathcal{U}_{\langle i\rangle}\right) \vDash$ " $\vec{u}^{i}$ is the pedigree for $f_{\langle i\rangle}$ over $\mathfrak{k}_{\Sigma \mathcal{U}_{\langle i\rangle}}(A) \wedge\left(\vec{u}^{i}\right)_{0}=\mathfrak{k}_{\Sigma \mathcal{U}_{\langle i\rangle}}\left(U^{i}\right)^{\prime}$, and let $\varphi_{i}: \mathcal{U}_{\langle i\rangle} \rightarrow \mathbf{U}^{i}$ be a morphism 
such that $\mathcal{U} \ell t\left(\mathbb{V} ; \mathcal{U}_{\langle i\rangle}\right) \vDash " \varphi_{i} \uparrow \Sigma T_{\mathcal{U}_{\langle i\rangle}}=\left(\vec{u}^{i}\right)^{+}$". Define $\vec{v}$ by $\vec{v}(\langle i\rangle \frown t)=\vec{u}^{i}(t)$ for $i \in I, t \in \Sigma T_{\mathcal{U}_{\langle i\rangle}} ;$ then $\vec{v} / T^{(1)}=\left\langle\vec{u}^{i}: i \in I\right\rangle$ and $\mathcal{U} \ell\left(\left\langle\mathcal{U} \ell t\left(\mathbb{V} ; \mathcal{U}_{\langle i\rangle}\right): i \in I\right\rangle ; \mathcal{U}(0)\right) \vDash$ " $\vec{v} / T^{(1)}$ is the pedigree for $f / T^{(1)}$ over $\mathfrak{k}_{\Sigma \mathcal{U}}(A) / T^{(1)}$ "; hence, by the Factoring Theorem, $\mathcal{U} \ell t(\mathbb{V} ; \mathcal{U}) \vDash$ " $\vec{v}$ is the $D_{T^{(1)}}$-pedigree for $f$ over $\mathfrak{k}_{\Sigma \mathcal{U}}(A)$ ".

Case 1 (collapsing)

There is some $U$ such that $U^{i}=U$ for all $i \in C \in \mathcal{U}(0)$.

Then $\mathcal{U} \ell t(\mathbb{V} ; \mathcal{U}) \vDash " v_{0}=\mathfrak{k}_{\Sigma \mathcal{U}}(U)$ ", and hence $\mathcal{U} \ell t(\mathbb{V} ; \mathcal{U}) \vDash " \vec{v}$ is the pedigree for $f$ over $\mathfrak{k}_{\Sigma \mathcal{U}}(A)$ ". We let $\vec{u}:=\vec{v}$ and define $\varphi$ by

$$
\left\{\begin{array}{l}
\varphi(0)=\varphi(\langle i\rangle)=0 \\
\varphi(\langle i\rangle \frown t)=\varphi_{i}(t)
\end{array}\right.
$$

for all $i \in C$ and $t \in \operatorname{dom} \varphi_{\langle i\rangle}$.

It is clear that $\varphi$ is a morphism of $\mathcal{U}$ to $\mathbf{U}$. We also have, for all $i \in C$ and $t \in \operatorname{dom} \varphi_{\langle i\rangle} \in \Sigma \mathcal{U}^{i}, \varphi(\langle i\rangle \frown t)=\varphi_{i}(t)=\left(\vec{u}^{i}\right)^{+}(t)=\vec{v}^{+}(\langle i\rangle \frown t)=\vec{u}^{+}(\langle i\rangle \frown t)$, so $\varphi \uparrow \Sigma T_{\mathcal{U}}=\Sigma \mathcal{U} \vec{u}^{+}$.

Case 2 (preserving)

Let $h: I \rightarrow \boldsymbol{\beta}_{\infty} A$ be defined by $h(i)=U^{i}$, and let $U \in \boldsymbol{\beta}_{\infty} A$ be such that $U \sim h[\mathcal{U}(0)]$ [see Proposition 9.2]; $U$ is nonprincipal. We let $\vec{u}(t)=\langle U\rangle \frown \vec{v}(t)$, for $t \in \Sigma T_{\mathcal{U}}$. It is easy to verify that $\mathcal{U} \ell t(\mathbb{V} ; \mathcal{U}) \vDash " \vec{u}$ is a pedigree for $f$ over $\mathfrak{k}_{\Sigma \mathcal{U}}(A)$ ". We define

$$
\left\{\begin{array}{l}
\varphi(0)=0 ; \\
\varphi(\langle i\rangle)=\left\langle U^{i}\right\rangle \text { for } i \in h^{-1}[\operatorname{dom} U] \in \mathcal{U}(0) \\
\varphi(\langle i\rangle \frown t)=\left\langle U^{i}\right\rangle \frown \varphi_{i}(t)
\end{array}\right.
$$

It is easy to verify that $\varphi$ is a morphism of $\mathcal{U}$ to $\mathbf{U}$. Also, for all $i \in I$ and $t \in \Sigma T_{\mathcal{U}(\langle i\rangle),} \varphi(\langle i\rangle \frown t)=\left\langle U^{i}\right\rangle \frown \varphi_{\langle i\rangle}(t)=\left\langle U^{i}\right\rangle \frown\left(\vec{u}^{i}\right)^{+}(t)=\left(\vec{u}^{i}\right)(t)=\vec{v}(\langle i\rangle \frown t)$ $=\vec{u}^{+}(\langle i\rangle \frown t)$.

Definition 10.22 (ZFC) Let $\mathfrak{M}_{1}$ and $\mathfrak{M}_{2}$ be realizations of SST $\mathbf{S T}^{b}$.

$\Phi$ is a morphism of $\mathfrak{M}_{1}$ to $\mathfrak{M}_{2}$ iff $\Phi$ is an $\in$-elementary embedding of $\mathfrak{M}_{1}$ to $\mathfrak{M}_{2}$ [in particular, $\left.\left(\forall x, y \in \mathfrak{M}_{1}\right)\left(x \sqsubseteq_{\mathfrak{M}_{1}} y \Leftrightarrow \Phi(x) \sqsubseteq_{\mathfrak{M}_{2}} \Phi(y)\right)\right]$ and $\Phi$ preserves good pedigrees, that is, $\left(\forall \alpha, x, \vec{u} \in \mathfrak{M}_{1}\right)(\vec{u}$ is a good $\alpha$-pedigree for $x \Rightarrow \Phi(\vec{u})$ is a good $\Phi(\alpha)$-pedigree for $\Phi(x))$.

Proposition 10.23 (ZFC) If $\varphi: \mathcal{U}_{2} \rightarrow \mathcal{U}_{1}$, then $\varphi^{*}: \mathcal{U} \ell t\left(\mathbb{V} ; \mathcal{U}_{1}\right) \rightarrow \mathcal{U} \ell t\left(\mathbb{V} ; \mathcal{U}_{2}\right)$ is a morphism. 
Proof This is a corollary of Proposition 10.15 and Proposition 10.5.

We prove that every morphism $\Phi: \mathcal{U} \ell t\left(\mathbb{V} ; \mathcal{U}_{1}\right) \rightarrow \mathcal{U} \ell t\left(\mathbb{V} ; \mathcal{U}_{2}\right)$ is of the form $\varphi^{*}$ for an essentially unique $\varphi: \mathcal{U}_{2} \rightarrow \mathcal{U}_{1}$.

Proposition 10.24 (ZFC) Let $\mathcal{U}$ be a TOU and $D:=D_{\mathcal{U}}:=D_{T_{\mathcal{U}}}$ be defined by $D(t)=t$ for $t \in A:=\Sigma T_{\mathcal{U}}$. If $\mathcal{U} \ell t(\mathbb{V} ; \mathcal{U}) \vDash " \vec{u}$ is the pedigree for $D$ over $\mathfrak{k}_{\Sigma \mathcal{U}}(A)$ and $u_{0}=\mathfrak{k}_{\Sigma \mathcal{U}}(U)$ ", and $\chi:=\chi_{\mathcal{U}}: \mathcal{U} \rightarrow \mathbf{U}$ is such that $\mathcal{U} \ell t(\mathbb{V} ; \mathcal{U}) \vDash \underline{\chi\left\lceil\Sigma T_{\mathcal{U}}\right.}=\vec{u}^{+}$, then $\chi$ is an isomorphism of $\mathcal{U}$ and $\mathbf{U}$.

Proof By induction on the rank of $\mathcal{U}$.

If $\mathcal{U}=0$, then $\mathbf{U}=0$ and the trivial morphism $\chi_{\mathcal{U}}$ is an isomorphism.

Assume that $T=\{0\} \cup \bigcup_{i \in I}\langle i\rangle \frown T_{i}$ and for each $i \in I, \mathcal{U} \ell t\left(\mathbb{V} ; \mathcal{U}_{\langle i\rangle}\right) \vDash " \vec{v}^{i}$ is the pedigree for $D^{i}$ over $A^{i}$ and $v_{0}^{i}=\mathfrak{k}_{\Sigma \mathcal{U}_{\langle i\rangle}}\left(V^{i}\right)$ ", where of course $D^{i}:=D_{\mathcal{U}_{\langle i\rangle}}$ and $A_{i}:=\Sigma T_{i}$, and $\widetilde{\chi}_{i}: \mathcal{U}_{\langle i\rangle} \rightarrow \mathbf{V}^{i}$ are the corresponding isomorphisms. We note that $\vec{v}^{i}(t)=\left\langle v_{0}^{i}(t), \ldots, v_{\nu_{i}(t)}^{i}(t)\right\rangle$ is a finite sequence of elements of $\boldsymbol{\beta}_{\infty} A_{i}$ for $\Sigma \mathcal{U}_{\langle i\rangle}$-almost all $t$. For $i \neq i^{\prime}$ we have $A_{i} \cap A_{i^{\prime}}=0$.

Let $\rho_{i}: A_{i} \rightarrow A$ be defined by $\rho_{i}(t)=\langle i\rangle \frown t$, and $\sigma_{i}: \subseteq A \rightarrow A_{i}$ by $\sigma_{i}(\langle i\rangle \frown t)=t$. It follows from the remark after Proposition 9.6 that $\overline{\rho_{i}}$ is an isomorphism of $V^{i} \in \boldsymbol{\beta}_{\infty} A_{i}$ and $\overline{\rho_{i}}\left(V_{i}\right) \in \boldsymbol{\beta}_{\infty} A$. We define $\vec{u}^{i}$ by $\vec{u}^{i}(t):=\left\langle\overline{\rho_{i}}\left(v_{0}^{i}(t)\right), \ldots, \overline{\rho_{i}}\left(v_{\nu_{i}(t)}^{i}(t)\right)\right\rangle$ and $\chi_{i}$ by $\chi_{i}(t):=\bar{\rho}_{i}\left(\widetilde{\chi}_{i}(t)\right)$; then $\mathcal{U} \ell t\left(\mathbb{V} ; \mathcal{U}_{\langle i\rangle}\right) \vDash " \vec{u}^{i}$ is the pedigree for $D_{\langle i\rangle}$ over $A$ and $u_{0}^{i}=\mathfrak{k}_{\Sigma \mathcal{U}_{\langle i\rangle}}\left(U^{i}\right) ", U^{i}=\overline{\rho_{i}}\left(V^{i}\right)$, and $\chi_{i}: \mathcal{U}_{\langle i\rangle} \rightarrow \mathbf{U}^{i}$ is an isomorphism [note that $\mathcal{U} \ell t\left(\mathbb{V} ; \mathcal{U}_{\langle i\rangle}\right) \vDash “ \vec{u}^{i}=\overline{\mathfrak{k}\left(\rho_{i}\right)} \circ \vec{v}^{i} \wedge \vec{v}^{i}=\overline{\mathfrak{k}\left(\sigma_{i}\right)} \circ \vec{u}^{i}$ ", and apply Theorem 10.10].

The mapping $h: i \mapsto U^{i}$ is one-one because $\overline{\rho_{i}}\left(V^{i}\right)$ concentrates on $\rho\left[A_{i}\right]$ and $\rho\left[A_{i}\right] \cap$ $\rho\left[A_{i^{\prime}}\right]=0$ for $i \neq i^{\prime}$ [see the remark following Definition 9.5]. Hence the preserving case in the proof of Proposition 10.21 occurs, $U \in \boldsymbol{\beta}_{\infty} A$ is defined by $U \sim h[\mathcal{U}(0)]$, and $\chi_{\mathcal{U}}(\langle i\rangle \frown t):=\langle U\rangle \frown \chi_{i}(t)$.

It is easily verified that $\chi_{\mathcal{U}}$ has the required properties.

Proposition 10.25 (ZFC) For every morphism $\Phi: \mathcal{U} \ell t\left(\mathbb{V} ; \mathcal{U}_{1}\right) \rightarrow \mathcal{U} \ell t\left(\mathbb{V} ; \mathcal{U}_{2}\right)$ there is $\varphi: \mathcal{U}_{2} \rightarrow \mathcal{U}_{1}$ such that $\Phi=\varphi^{*}$. If also $\varphi^{\prime}: \mathcal{U}_{2} \rightarrow \mathcal{U}_{1}$ and $\Phi=\left(\varphi^{\prime}\right)^{*}$, then $\varphi \uparrow T=\varphi^{\prime} \uparrow T$ for some maximal $T \preccurlyeq \mathcal{U}_{2} T_{\mathcal{U}_{2}}$.

Proof Apply Proposition 10.24 to $\mathcal{U}^{1}$ and $D_{\mathcal{U}^{1}}$ to obtain $\vec{u}^{1}, U^{1}$, and an isomorphism $\chi:=\chi_{\mathcal{U}^{1}}: \mathcal{U}^{1} \rightarrow \mathbf{U}^{1}$. Since $\Phi$ is a morphism, we have $\mathcal{U} \ell t\left(\mathbb{V} ; \mathcal{U}^{2}\right) \vDash " \Phi\left(\vec{u}^{1}\right)$ is the pedigree for $\Phi\left(D_{\mathcal{U}^{1}}\right)$ over $\mathfrak{k}_{\Sigma \mathcal{U}^{2}}(A) \wedge \Phi\left(u_{0}^{1}\right)=\mathfrak{k}_{\Sigma \mathcal{U}^{2}}\left(U^{1}\right)$ ". By Proposition 10.21 there 
is a morphism $\psi: \mathcal{U}^{2} \rightarrow \mathbf{U}^{1}$. The composition $\varphi:=\chi^{-1} \circ \psi$ is then a morphism of $\mathcal{U}^{2}$ to $\mathcal{U}^{1}$.

We have $\psi^{*}\left(E_{\mathbf{U}^{1}}\right)=_{\Sigma \mathcal{U}^{2}} \Phi\left(D_{\mathcal{U}^{1}}\right)$ and $\chi^{*}\left(E_{\mathbf{U}^{1}}\right)=_{\Sigma \mathcal{U}^{1}} D_{\mathcal{U}^{1}}$, by Proposition 10.21. Hence $\Phi\left(D_{\mathcal{U}^{1}}\right)=_{\Sigma \mathcal{U}^{2}} \psi^{*}\left(E_{\mathbf{U}^{1}}\right)=_{\Sigma \mathcal{U}^{2}} \psi^{*}\left(\left(\chi^{*}\right)^{-1}\left(D_{\mathcal{U}^{1}}\right)\right)=_{\Sigma \mathcal{U}^{2}} \varphi^{*}\left(D_{\mathcal{U}^{1}}\right)=_{\Sigma \mathcal{U}^{2}} D_{\mathcal{U}^{1}} \circ$ $\left(\varphi \uparrow \Sigma T_{\mathcal{U}^{2}}\right)={ }_{\Sigma \mathcal{U}^{2}} \varphi \uparrow \Sigma T_{\mathcal{U}^{2}}$.

For all $f \in \mathbb{V}^{\Sigma \mathcal{U}^{1}}$ we have, as in the proof of Proposition 2.5 Claim 2, that $\mathcal{U} \ell t\left(\mathbb{V} ; \mathcal{U}_{1}\right) \vDash$ $f=\mathfrak{k}_{\Sigma \mathcal{U}^{1}}(f)\left(D_{\mathcal{U}^{1}}\right)$, hence $\mathcal{U} \ell t\left(\mathbb{V} ; \mathcal{U}_{2}\right) \vDash \Phi(f)=\mathfrak{k}_{\Sigma \mathcal{U}^{2}}(f)\left(\Phi\left(D_{\mathcal{U}^{1}}\right)\right)$. We finally conclude that $\Phi(f)=_{\Sigma \mathcal{U}^{2}} f \circ \Phi\left(D_{\mathcal{U}^{1}}\right)=_{\Sigma \mathcal{U}^{2}} f \circ\left(\varphi \mid \Sigma T_{\mathcal{U}^{2}}\right)=_{\Sigma \mathcal{U}^{2}} \varphi^{*}(f)$.

Assume now that $\varphi: \mathcal{U}^{2} \rightarrow \mathcal{U}^{1}, \varphi^{\prime}: \mathcal{U}^{2} \rightarrow \mathcal{U}^{1}$, and $\Phi=\varphi^{*}=\left(\varphi^{\prime}\right)^{*}$. It follows that $\varphi\left\lceil\Sigma T_{\mathcal{U}^{2}}=_{\Sigma \mathcal{U}^{2}} \varphi^{*}\left(D_{\mathcal{U}^{1}}\right)=_{\Sigma \mathcal{U}^{2}}\left(\varphi^{\prime}\right)^{*}\left(D_{\mathcal{U}^{1}}\right)=_{\Sigma \mathcal{U}^{2}} \varphi^{\prime} \uparrow \Sigma T_{\mathcal{U}^{2}}\right.$. The conclusion follows by Proposition 6.16 .

\section{Stratified Limit Ultrapowers.}

\subsection{Stratified limit ultrapowers.}

A directed system of TOUs consists of the following data: a directed preordering $(\mathbb{D}, \leq)$, a system of TOUs $\mathbb{U}=\left\langle\mathcal{U}_{d}: d \in \mathbb{D}\right\rangle$, and a system $\phi=\left\langle\phi_{d, d^{\prime}}: d \leq d^{\prime}\right\rangle$ such that, for all $d \leq d^{\prime} \leq d^{\prime \prime}, \phi_{d, d^{\prime}} \neq 0, \varphi \in \phi_{d, d^{\prime}} \Rightarrow \varphi: \mathcal{U}_{d^{\prime}} \rightarrow \mathcal{U}_{d}, \varphi, \varphi^{\prime} \in$ $\phi_{d, d^{\prime}} \Rightarrow \varphi=\Sigma \mathcal{U}_{d^{\prime}} \varphi^{\prime}$, and $\varphi \in \phi_{d, d^{\prime}}, \varphi^{\prime} \in \phi_{d^{\prime}, d^{\prime \prime}}$ implies $\varphi \circ \varphi^{\prime}=\Sigma \mathcal{U}_{d^{\prime \prime}} \varphi^{\prime \prime}$ for some $\varphi^{\prime \prime} \in \phi_{d, d^{\prime \prime}}$.

A directed system of TOUs induces a system of interpretations $\left\langle\mathcal{U} \ell t\left(\mathbb{V} ; \mathcal{U}_{d}\right): d \in \mathbb{D}\right\rangle$, and a system $\Phi=\left\langle\Phi_{d, d^{\prime}}: d \leq d^{\prime}\right\rangle$ of morphisms $\Phi_{d, d^{\prime}}: \mathcal{U} \ell t\left(\mathbb{V} ; \mathcal{U}_{d}\right) \rightarrow \mathcal{U} \ell t\left(\mathbb{V} ; \mathcal{U}_{d^{\prime}}\right)$ that commute with the canonical embeddings: $\Phi_{d, d^{\prime}} \circ \mathfrak{k}_{\Sigma \mathcal{U}_{d}}=\mathfrak{k}_{\Sigma \mathcal{U}_{d^{\prime}}}$, and such that $d \leq d^{\prime} \leq d^{\prime \prime} \Rightarrow \Phi_{d, d^{\prime \prime}}=\Phi_{d^{\prime}, d^{\prime \prime}} \circ \Phi_{d, d^{\prime}}$; namely, $\Phi_{d, d^{\prime}}=\varphi^{*}$ for any $\varphi \in \phi_{d, d^{\prime}}$ [it is independent of the choice of $\varphi$ ].

The stratified limit ultrapower of the universe $\mathcal{L U} \ell t(\mathbb{V} ; \mathbb{U}, \phi)$ is the direct limit of this system.

For a more concrete description of this interpretation, we observe that the given data induces a directed system of ultrafilters $\widetilde{\mathbb{U}}:=\left\langle\Sigma \mathcal{U}_{d}: d \in \mathbb{D}\right\rangle, \quad \widetilde{\phi}:=\left\langle\widetilde{\phi}_{d, d^{\prime}}: d \leq d^{\prime}\right\rangle$, where $\widetilde{\phi}_{d, d^{\prime}}:=\left\{\varphi \mid \Sigma T_{\mathcal{U}_{d}^{\prime}}: \varphi \in \phi_{d, d^{\prime}}\right\}$.

The limit ultrapower $\mathcal{L U} \ell t(\mathbb{V} ; \widetilde{\mathbb{U}}, \widetilde{\phi})$ described in Section 5 is a realization of $\mathbf{S T}$, and each $\Phi_{d, *}: \mathcal{U} \ell t\left(\mathbb{V} ; \Sigma \mathcal{U}_{d}\right) \rightarrow \mathcal{L U} \ell t(\mathbb{V} ; \widetilde{\mathbb{U}}, \widetilde{\phi})$ is a morphism of interpretations for the 
$\{\in, \mathbf{s t}\}$-language, that is, an $\in$-elementary embedding commuting with the canonical embeddings [Proposition 5.1].

The interpretations $\mathcal{L U} \ell t(\mathbb{V} ; \mathbb{U}, \phi)$ and $\mathcal{L U} \ell t(\mathbb{V} ; \widetilde{\mathbb{U}}, \widetilde{\phi})$ have the same universe, the same $\epsilon^{*}$ and $=^{*}$ [interpretations of $\in$ and $=$, resp.] and the same $\Phi_{d, *}$.

The relation $\sqsubseteq$ is interpreted in $\mathcal{L U} \ell t(\mathbb{V} ; \mathbb{U}, \phi)$ by $\sqsubseteq^{*}$, where $f \sqsubseteq^{*} g$ iff $\left(\exists d^{\prime \prime} \in \mathbb{D}\right)(d \leq$ $\left.d^{\prime \prime} \wedge d^{\prime} \leq d^{\prime \prime} \wedge \Phi_{d, d^{\prime \prime}}(f) \sqsubseteq \mathcal{U}_{d^{\prime \prime}} \Phi_{d^{\prime}, d^{\prime \prime}}(g)\right)$, for $f \in \mathbb{V}^{\Sigma \mathcal{U}_{d}}, g \in \mathbb{V}^{\Sigma \mathcal{U}_{d^{\prime}}}$.

We note that $f \in \mathbb{S}_{0}^{*} \Leftrightarrow f={ }^{*} \mathfrak{k}_{\Sigma \mathcal{U}_{d}}(c)$ for some $c \in \mathbb{V}$, and $\Phi_{d, *}$ preserve $\sqsubseteq$, that is, for $f, g \in \mathbb{V}^{\Sigma \mathcal{U}_{d}}, f \sqsubseteq \mathcal{U}_{d} g \Leftrightarrow f \sqsubseteq^{*} g$.

Theorem 11.1 $\mathcal{L U} \ell t(\mathbb{V} ; \mathbb{U}, \phi)$ is a realization of $\mathbf{S S T}^{b}$.

For each $d \in \mathbb{D}, \Phi_{d, *}: \mathcal{U} \ell t\left(\mathbb{V} ; \mathcal{U}_{d}\right) \rightarrow \mathcal{L U} \ell t(\mathbb{V} ; \mathbb{U}, \phi)$ is a morphism.

Proof The axiom of Relativization follows immediately from the definition of $\complement^{*}$ and the fact that it is satisfied by each $\sqsubseteq \mathcal{U}_{d}$.

\section{Transfer:}

We prove that, for any $\in$-formula $\mathcal{P}$ and any $f_{1}, \ldots, f_{k} \sqsubseteq^{*} h, \quad \mathcal{L U} \ell t(\mathbb{V} ; \mathbb{U}, \phi) \vDash$ $(\exists g) \mathcal{P}(g, \bar{f})$ implies $\mathcal{L U} \ell t(\mathbb{V} ; \mathbb{U}, \phi) \vDash(\exists g \sqsubseteq h) \mathcal{P}(g, \bar{f})$. Transfer into $\mathbb{S}_{\alpha}$ then follows by the usual induction on the complexity of $\mathcal{P}$.

Let $h \in \mathbb{V}^{\Sigma \mathcal{U}_{d}}, f_{i} \in \mathbb{V}^{\Sigma \mathcal{U}_{d_{i}}}$, and $f_{i} \sqsubseteq^{*} h$ for all $i$. From the definition of $\sqsubseteq^{*}$ and the facts that all $\Phi_{d, d^{\prime}}$ preserve $\sqsubseteq$ and $(\mathbb{D}, \leq)$ is directed, we get $d \geq \widetilde{d}, d_{i}$ and $g \in \mathbb{V}^{\Sigma \mathcal{U}_{d}}$ such that $\mathcal{U} \ell t\left(\mathbb{V} ; \mathcal{U}_{d}\right) \vDash \Phi_{d_{i}, d}\left(f_{i}\right) \sqsubseteq \Phi_{\widetilde{d}, d}(h)$ and $\mathcal{L U} \ell t(\mathbb{V} ; \mathbb{U}, \phi) \vDash \mathcal{P}(g, \bar{f})$. As $\Phi_{d, *}$ is an $\in$-elementary embedding, $\mathcal{U} \ell t\left(\mathbb{V} ; \mathcal{U}_{d}\right) \vDash \mathcal{P}\left(g, \Phi_{d_{1}, d}\left(f_{1}\right), \ldots, \Phi_{d_{k}, d}\left(f_{k}\right)\right)$. By Proposition 8.1, $\mathcal{U} \ell t\left(\mathbb{V} ; \mathcal{U}_{d}\right) \vDash \mathbf{S S T}$, and it follows that there is $\widetilde{g} \in \mathbb{V}^{\Sigma \mathcal{U}_{d}}$ such that $\mathcal{U} \ell t\left(\mathbb{V} ; \mathcal{U}_{d}\right) \vDash \widetilde{g} \sqsubseteq \Phi_{\widetilde{d}, d}(h) \wedge \mathcal{P}\left(\widetilde{g}, \Phi_{d_{1}, d}\left(f_{1}\right), \ldots, \Phi_{d_{k}, d}\left(f_{k}\right)\right)$. Applying the $\sqsubseteq-$ preserving, $\in$-elementary $\Phi_{d, *}$, we get $\mathcal{L U} \ell t(\mathbb{V} ; \mathbb{U}, \phi) \vDash \widetilde{g} \sqsubseteq h \wedge \mathcal{P}(\widetilde{g}, \bar{f})\left[\Phi_{d, *}(\widetilde{g})=\right.$ $\widetilde{g}, \Phi_{d, *}\left(\Phi_{\widetilde{d}, d}(h)\right)={ }^{*} \Phi_{\widetilde{d}, *}(h)=h$, etc. $]$, ie, $\mathcal{L U} \ell t(\mathbb{V} ; \mathbb{U}, \phi) \vDash(\exists g \sqsubseteq h) \mathcal{P}(g, \bar{f})$.

$(\forall \alpha)\left(\mathbf{B}_{\alpha}\right)$ :

Lemma 11.2 If $\mathcal{U} \ell t\left(\mathbb{V} ; \mathcal{U}_{d}\right) \vDash$ " $\vec{u}$ is an $h$-pedigree for $f$ over $A \sqsubseteq h$ ", then $\mathcal{L U} \ell t(\mathbb{V} ; \mathbb{U}, \phi) \vDash$ “ $\vec{u}$ is a good $h$-pedigree for $f$ over $A \sqsubseteq h$ ".

Proof $\mathcal{L U} \ell t(\mathbb{V} ; \mathbb{U}, \phi)$ is an $\in$-elementary extension of $\mathcal{U} \ell\left(\mathbb{V} ; \mathcal{U}_{d}\right)$, and $\sqsubseteq \mathcal{U}_{d}$ is the restriction of $\sqsubseteq^{*}$ to $\mathbb{V}^{\Sigma \mathcal{U}_{d}}$. From these observations it immediately follows that conditions (i) and (ii) from the definition of pedigree hold in $\mathcal{L U} \ell t(\mathbb{V} ; \mathbb{U}, \phi)$. 
Let $\mathcal{L U} \ell t(\mathbb{V} ; \mathbb{U}, \phi) \vDash " n<m \wedge X \in u_{n} \wedge X \sqsubset_{h} u_{n+1}$ ". Without loss of generality $n, m, X \in \mathbb{V}^{\Sigma \mathcal{U}_{d^{\prime}}}$ for some $d^{\prime} \geq d$. By Proposition 10.15, $\mathcal{U} \ell t\left(\mathbb{V} ; \mathcal{U}_{d^{\prime}}\right) \vDash “ \Phi_{d, d^{\prime}}(\vec{u})$ is a $\Phi_{d, d^{\prime}}(h)$-pedigree for $\Phi_{d, d^{\prime}}(f)$ over $\Phi_{d, d^{\prime}}(A)$ ". In particular, $\mathcal{U} \ell t\left(\mathbb{V} ; \mathcal{U}_{d^{\prime}}\right) \vDash$ $\Phi_{d, d^{\prime}}(\vec{u})_{n} \sqsubset \Phi_{d, d^{\prime}}(h) \Phi_{d, d^{\prime}}(\vec{u})_{m}$ [where $\Phi_{d, d^{\prime}}(\vec{u})_{n}$ denotes the $n$-th term of the finite sequence $\Phi_{d, d^{\prime}}(\vec{u})$ in the sense of $\left.\mathcal{U} \ell t\left(\mathbb{V} ; \mathcal{U}_{d^{\prime}}\right)\right]$, and so, applying $\Phi_{d^{\prime}, *}$ and observing that $\Phi_{d^{\prime}, *}\left(\Phi_{d, d^{\prime}}(\vec{u})_{n}\right)=u_{n}$ etc, $\mathcal{L U} \ell t(\mathbb{V} ; \mathbb{U}, \phi) \vDash u_{n} \sqsubset_{h} u_{m}$. This proves condition (iii).

Similarly, $\mathcal{U} \ell t\left(\mathbb{V} ; \mathcal{U}_{d^{\prime}}\right) \vDash \Phi_{d, d^{\prime}}(\vec{u})_{n+1} \in X$, and hence $\mathcal{L U} \ell t(\mathbb{V} ; \mathbb{U}, \phi) \vDash u_{n+1} \in X$. This proves condition (iv).

It remains to verify that $\mathcal{L U} \ell t(\mathbb{V} ; \mathbb{U}, \phi)$ satisfies the condition (j).

Let $X, g, g^{\prime}$ be such that $\mathcal{L U} \ell t(\mathbb{V} ; \mathbb{U}, \phi) \vDash “ X, g, g^{\prime} \sqsubseteq h \wedge X \in \Sigma \mathbf{u}_{0} \wedge g, g^{\prime} \in \mathbb{V}^{\Sigma \mathbf{u}_{0}}$ ". Without loss of generality $X, g, g^{\prime} \in \mathbb{V}^{\Sigma \mathcal{U}_{d^{\prime}}}$ for some $d^{\prime} \geq d$ and $\mathcal{U} \ell t\left(\mathbb{V} ; \mathcal{U}_{d^{\prime}}\right) \vDash$ “ $X, g, g^{\prime} \sqsubseteq \Phi_{d, d^{\prime}}(h) \wedge X \in \Sigma \Phi_{d, d^{\prime}}\left(\mathbf{u}_{0}\right)$ ". As $\Phi_{d, d^{\prime}}(\vec{u})$ is a good $\Phi_{d, d^{\prime}}(h)$-pedigree in $\mathcal{U} \ell t\left(\mathbb{V} ; \mathcal{U}_{d^{\prime}}\right), \quad \mathcal{U} \ell t\left(\mathbb{V} ; \mathcal{U}_{d^{\prime}}\right) \vDash “ \Phi_{d, d^{\prime}}\left(\vec{u}^{+}\right) \in X \wedge\left[g \sqsubseteq_{\Phi_{d, d^{\prime}}}\left(\mathbf{u}_{0}\right) g^{\prime} \Leftrightarrow g\left(\Phi_{d, d^{\prime}}\left(\vec{u}^{+}\right)\right) \sqsubseteq \Phi_{d, d^{\prime}}(h)\right.$ $\left.g^{\prime}\left(\Phi_{d, d^{\prime}}\left(\vec{u}^{+}\right)\right)\right] ”$. Applying $\Phi_{d^{\prime}, *}, \quad \mathcal{L} \mathcal{U} \ell t(\mathbb{V} ; \mathbb{U}, \phi) \vDash “ \vec{u}^{+} \in X \wedge\left(g \sqsubseteq \mathbf{u}_{0} g^{\prime} \Leftrightarrow g\left(\vec{u}^{+}\right) \sqsubseteq_{h}\right.$ $\left.g^{\prime}\left(\vec{u}^{+}\right)\right)$.

The proof of Lemma 11.2 shows that $\Phi_{d, *}$ takes pedigrees in $\mathcal{U} \ell t\left(\mathbb{V} ; \mathcal{U}_{d}\right)$ into good pedigrees in $\mathcal{L U} \ell t(\mathbb{V} ; \mathbb{U}, \phi)$; ie, that $\Phi_{d, *}$ is a morphism.

Finally, let $\mathcal{L U} \ell t(\mathbb{V} ; \mathbb{U}, \phi) \vDash " f \mathbb{M}_{h} U \wedge U \in \boldsymbol{\beta}_{\infty}(A) \wedge f=F(g) \wedge A, F \sqsubseteq h$ ". We fix $d$ so that $f, g, h, A, F \in \mathbb{V}^{\Sigma \mathcal{U}_{d}}$. Let $\mathcal{U} \ell t\left(\mathbb{V} ; \mathcal{U}_{d}\right) \vDash$ " $\vec{u}$ is a good $h$-pedigree for $f$ over $A$ ". By Lemma 11.2, $\mathcal{L} \mathcal{U} \ell t(\mathbb{V} ; \mathbb{U}, \phi)$ satisfies this statement as well, and, by uniqueness of pedigrees [true in $\mathcal{L U} \ell t(\mathbb{V} ; \mathbb{U}, \phi) \vDash \mathbf{S S T}], \quad \mathcal{L U} \ell t(\mathbb{V} ; \mathbb{U}, \phi) \vDash U=u_{0}$, hence also $\mathcal{U} \ell t\left(\mathbb{V} ; \mathcal{U}_{d}\right) \vDash U=u_{0}$.

There exists $\vec{v}$ so that

(*) $\quad \mathcal{U} \ell t\left(\mathbb{V} ; \mathcal{U}_{d}\right) \vDash " v \vec{v}$ is a good $h$-pedigree for $g$ over $B$, where $B \sqsubseteq h$ ".

Theorem 10.10 holds in $\mathcal{U} \ell t\left(\mathbb{V} ; \mathcal{U}_{d}\right)$, so

$$
\mathcal{U} \ell t\left(\mathbb{V} ; \mathcal{U}_{d}\right) \vDash u_{0}=\bar{F}\left(v_{0}\right) .
$$

By Lemma 11.2 and $\Phi_{d, *}$ being $\in$-elementary, (*) and (**) hold in $\mathcal{L U} \ell t(\mathbb{V} ; \mathbb{U}, \phi)$. So, letting $V:=v_{0}$, we have $\mathcal{L U} \ell t(\mathbb{V} ; \mathbb{U}, \phi) \vDash “ V \sqsubseteq h \wedge U=\bar{F}(V) \wedge g \mathbb{M}_{h} V$." This shows validity of $(\forall \alpha)\left(\mathbf{B}_{\alpha}\right)$ in $\mathcal{L U} \ell t(\mathbb{V} ; \mathbb{U}, \phi)$.

Inner Standardization is a consequence of $(\forall \alpha)\left(\mathbf{B}_{\alpha}\right)$, as pointed out in the remark following Definition 10.13.

Theorem 11.3 Every realization of $\mathbf{S S T}^{b}$ is isomorphic to a stratified limit ultrapower of the universe. 
This theorem is not used anywhere in the paper, and we omit its proof, which follows the lines of the proof of Proposition 5.2, with $\mathbb{S}[[x]]$ in place of $\mathbb{S}[x]$.

\subsection{Internally iterated ultrapowers.}

The next subsection is devoted to the construction of a special kind of stratified limit ultrapower. The technique used in this construction was developed in [13] and called there internally iterated ultrapower.

We fix a linearly ordered set $\langle\Lambda, \leq\rangle$. For $\alpha \in \Lambda, \Lambda(>\alpha):=\{\beta \in \Lambda: \alpha<\beta\}$. Let $S e q_{\Lambda}^{I}:=\bigcup\left\{I^{F}: F \subseteq \Lambda, F\right.$ finite $\}$. For $s \in \operatorname{Seq}_{\Lambda}^{I}, \bar{s}_{\Lambda}^{I}:=\left\{f \in I^{\Lambda}: s \subseteq f\right\}$.

We say that $P \subseteq S e q_{\Lambda}^{I}$ is a partition if $\left(\forall f \in I^{\Lambda}\right)(\exists ! s \in P)(s \subseteq f)$, ie, $\bar{P}:=\left\{\bar{s}_{\Lambda}^{I}: s \in\right.$ $P\}$ is a partition of $I^{\Lambda}$ in the usual sense. For a partition $P, \operatorname{dom} P:=\bigcup_{s \in P} \operatorname{dom} s$; $\min P:=$ the least element of $\operatorname{dom} P$, if it exists. [By convention, the least (greatest, resp.) element of the empty set is $+\infty(-\infty$, resp.), so if $P=\{0\}, \min P=+\infty$ and $\max P=-\infty$.]

Let $P, Q$ be partitions; by definition, $P \unlhd Q$ iff $(\forall t \in Q)(\exists s \in P)(s \subseteq t)$; we denote this unique $s$ by $\pi_{P, Q}(t)$. We then say that $Q$ is a refinement of $P$ and $\pi_{P, Q}$ is the projection of $Q$ onto $P$. Clearly $\unlhd$ is an ordering, $\pi_{P, P}=I d_{P}, \pi_{P, Q} \circ \pi_{Q, R}=\pi_{P, R}$ for $P \unlhd Q \unlhd R$. For any $P, Q$ let $P \nabla Q:=\left\{s \cup t \in S e q_{\Lambda}^{I}: s \in P, t \in Q\right\}$. It is easy to check that $P \nabla Q$ is a partition, $P, Q \unlhd P \nabla Q$ and $P, Q \unlhd R \Rightarrow P \nabla Q \unlhd R$.

We define $\nu_{P}: P \rightarrow \Lambda$ by $\nu_{P}(s)=\max (\operatorname{dom} s)$. We say $\left\langle P_{s}: s \in P\right\rangle$ is summable if $P \subseteq S e q_{\Lambda}^{I}$ is a partition and for each $s \in P, P_{s} \subseteq S e q_{\Lambda\left(>\nu_{P}(s)\right)}^{I}$ is a partition. Then $Q:=\Sigma_{s \in P} P_{s}:=\left\{s \cup t: s \in P, t \in P_{s}\right\}$ is a partition, $\operatorname{dom} Q=\operatorname{dom} P \cup$ $\bigcup_{s \in P} \operatorname{dom} P_{s}, \min Q=\min P$.

Definition 11.4 $\mathcal{F}_{\Lambda}^{I}$ is the least collection of partitions of $S e q_{\Lambda}^{I}$ containing the trivial partition $\{0\}$ and all partitions $P^{\alpha}:=P^{\alpha, I}:=\{\{(\alpha, i)\}: i \in I\}$ for $\alpha \in \Lambda$, and closed under $\Sigma$. In detail, we construct by transfinite recursion:

$\mathcal{F}_{\Lambda}^{I}(0):=\{\{0\}\}$

$\mathcal{F}_{\Lambda}^{I}(\xi+1):=\mathcal{F}_{\Lambda}^{I}(\xi) \cup\left\{\Sigma_{a \in P^{\alpha}} P_{a}: \alpha \in \Lambda \wedge\left(\forall a \in P^{\alpha}\right)\left[P_{a} \in \mathcal{F}_{\Lambda(>\alpha)}^{I}(\xi)\right]\right\}$

$\mathcal{F}_{\Lambda}^{I}(\eta):=\bigcup_{\xi<\eta} \mathcal{F}_{\Lambda}^{I}(\xi)$ for $\eta$ limit

$\mathcal{F}_{\Lambda}^{I}:=\bigcup_{\xi \in \mathbb{O} n} \mathcal{F}_{\Lambda}^{I}(\xi)$.

We note that the construction always terminates; in fact, $\mathcal{F}_{\Lambda}^{I}(\xi+1)=\mathcal{F}_{\Lambda}^{I}(\xi)$ for $\xi=\kappa^{+}$, where $|I|=\kappa$. For $P \in \mathcal{F}_{\Lambda}^{I}$, rank $P$ is the least $\xi$ such that $P \in \mathcal{F}_{\Lambda}^{I}(\xi)$. 
Proposition 11.5 (i) $P^{\alpha, I} \in \mathcal{F}_{\Lambda}^{I}$ for all $\alpha \in \Lambda$.

(ii) If $P \in \mathcal{F}_{\Lambda}^{I}, P_{s} \in \mathcal{F}_{\Lambda}^{I}$ for all $s \in P$, and $\left\langle P_{s}: s \in P\right\rangle$ is summable, then $\Sigma_{s \in P} P_{s} \in \mathcal{F}_{\Lambda}^{I}$.

Proof (i) $P^{\alpha}=\Sigma_{a \in P^{\alpha}}\{0\} \in \mathcal{F}_{\Lambda}(1)$.

(ii) We proceed by induction on $\operatorname{rank} P$. Let $P=\Sigma_{a \in P^{\alpha}} P_{a}$; by inductive assumption, for each $a \in P^{\alpha}, \quad \Sigma_{t \in P_{a}} P_{a \cup t} \in \mathcal{F}_{\Lambda}$. Hence $\Sigma_{s \in P} P_{s}=\Sigma_{a \in P^{\alpha}}\left(\Sigma_{t \in P_{a}} P_{a \cup t}\right) \in \mathcal{F}_{\Lambda}$. [Note that every $s \in P$ uniquely decomposes as $a \cup t$ where $a \in P^{\alpha}, \alpha<\min t, t \in$ $P_{a}$.]

Let $U$ be an ultrafilter over $I . U_{\alpha}:=j_{\alpha}[U]$ where $j_{\alpha}: I \rightarrow P^{\alpha}$ is the isomorphism defined by $j_{\alpha}(i)=\{(\alpha, i)\}$. We have $A \in U_{\alpha} \Leftrightarrow\{i \in I:\{(\alpha, i)\} \in A\} \in U$, for all $A \subseteq P^{\alpha}$.

We define an ultrafilter $U_{P}$ over $P \in \mathcal{F}_{\Lambda}^{I}$ by recursion:

$U_{\{0\}}$ is the principal ultrafilter over $\{0\}$.

If $P=\Sigma_{a \in P^{\alpha}} P_{a}$, then, for $A \subseteq P, A \in U_{P} \Leftrightarrow\left\{a \in P^{\alpha}:\left\{s \in P_{a}: a \cup s \in A\right\} \in\right.$ $\left.U_{P_{a}}\right\} \in U_{\alpha}$. [Hence, $U_{P}$ is isomorphic to $\Sigma_{U_{\alpha}} U_{P_{a}}$ via the map $a \cup s \mapsto\langle a, s\rangle$.]

From associativity of the Rudin-Frolík sum we get by induction: if $Q=\Sigma_{s \in P} P_{s}$, then $U_{Q} \cong \Sigma_{U_{P}} U_{P_{s}}$.

Proposition 11.6 If $P, Q \in \mathcal{F}_{\Lambda}^{I}$, then $P \nabla Q \in \mathcal{F}_{\Lambda}^{I}$ and $U_{P}=\pi_{P, P \nabla Q}\left[U_{P \nabla Q}\right]$,

$U_{Q}=\pi_{Q, P \nabla Q}\left[U_{P \nabla Q}\right]$.

Proof By double induction on rank. We assume that the claim holds for all $P \in \mathcal{F}_{\Lambda}$ of rank less than $\xi$ and all $Q \in \mathcal{F}_{\Lambda}$. We prove that it then holds if $\operatorname{rank} P=\xi$ and $Q \in \mathcal{F}_{\Lambda}$, by induction on $\eta:=\operatorname{rank} Q$.

The case when either $P$ or $Q$ is $\{0\}$ is trivial, so let $P=\Sigma_{a \in P^{\alpha}} P_{a}, Q=\Sigma_{b \in P^{\beta}} Q_{b}$.

From the definition of $\nabla$ one sees that:

If $\alpha=\beta$, then $P \nabla Q=\Sigma_{a \in P^{\alpha}}\left(P_{a} \nabla Q_{a}\right)$.

If $\alpha<\beta$, then $P \nabla Q=\Sigma_{a \in P^{\alpha}}\left(P_{a} \nabla Q\right)$.

If $\alpha>\beta$, then $P \nabla Q=\Sigma_{b \in P^{\beta}}\left(P \nabla Q_{b}\right)$.

In each case the terms of the sum belong to $\mathcal{F}_{\Lambda}$ by inductive assumption, hence $P \nabla Q \in \mathcal{F}_{\Lambda}$. 
To prove the claim about ultrafilters, we work out the case $\alpha<\beta$ in detail. If $A \subseteq P$ or $A \subseteq P \nabla Q$, and $a \in P_{\alpha}$, we let $A_{a}:=\{s: a \cup s \in A\}$.

We now have: $A \in U_{P} \Rightarrow\left\{a \in P^{\alpha}: A_{a} \in U_{P_{a}}\right\} \in U_{\alpha} \Rightarrow$ [by the inductive assumption $]\left\{a \in P^{\alpha}: \pi_{P_{a}, P_{a} \nabla Q}^{-1}\left[A_{a}\right] \in U_{P_{a} \nabla Q}\right\} \in U_{\alpha} \Rightarrow\left\{a \in P^{\alpha}:\left(\pi_{P, P \nabla Q}^{-1}[A]\right)_{a} \in\right.$ $\left.U_{P_{a} \nabla Q}\right\} \in U_{\alpha} \Rightarrow \pi_{P, P \nabla Q}^{-1}[A] \in U_{P \nabla Q}$.

Similarly: $B \in U_{Q} \Rightarrow$ [by the inductive assumption] $\left(\forall a \in P^{\alpha}\right)\left(\pi_{Q, P_{a} \nabla Q}^{-1}[B] \in\right.$ $\left.U_{P_{a} \nabla Q}\right) \Rightarrow\left\{a \in P^{\alpha}: \pi_{Q, P_{a} \nabla Q}^{-1}[B] \in U_{P_{a} \nabla Q}\right\} \in U_{\alpha} \Rightarrow\left\{a \in P^{\alpha}:\left(\pi_{Q, P \nabla Q}^{-1}[B]\right)_{a} \in\right.$ $\left.U_{P_{a} \nabla Q}\right\} \in U_{\alpha} \Rightarrow \pi_{Q, P \nabla Q}^{-1}[B] \in U_{P \nabla Q}$.

Corollary 11.7 If $P \unlhd Q$, then $U_{P}=\pi_{P, Q}\left[U_{Q}\right]$.

Proof $P \unlhd Q$ implies $P \nabla Q=Q$.

With each partition $P \in \mathcal{F}_{\Lambda}^{I}$ there is associated a uniquely determined tree $T_{P}$ in the sense of Section 6.

Let $\Gamma: \operatorname{Seq}_{\Lambda}^{I} \rightarrow \mathbb{V}<\omega$ be the one-one mapping defined by $\Gamma(s)=t$, where $s=$ $\left\{\left(\alpha_{0}, i_{0}\right), \ldots,\left(\alpha_{k-1}, i_{k-1}\right)\right\} \in \operatorname{Seq}_{\Lambda}^{I}$ and $t=\left\langle\left(\alpha_{0}, i_{0}\right), \ldots,\left(\alpha_{k-1}, i_{k-1}\right)\right\rangle$, with $\alpha_{0}<$ $\ldots<\alpha_{k-1}$. [For our purposes, this is more suitable than the simpler mapping $\left.s \mapsto\left\langle i_{0}, \ldots, i_{k-1}\right\rangle.\right]$

The tree $T_{P}$ is obtained by replacing each $s \in P$ by $\Gamma(s)$, and closing under subsequences. A more formal definition is by recursion:

If $P=\{0\}$, then $T_{P}=\{0\}$.

If $P=\Sigma_{a \in P^{\alpha}} P_{a}$, and for each $a=\{(\alpha, i)\}, T_{i}:=T_{P_{a}}$ is the tree associated to $P_{a}$, then $T_{P}:=\{0\} \cup \bigcup_{i \in I}\langle(\alpha, i)\rangle \frown T_{i}$.

With some license, we use $a$ to denote $\{(\alpha, i)\},\langle(\alpha, i)\rangle$, or $(\alpha, i)$, whichever is appropriate.

The elements of the partition $P$ are in one-one correspondence with the leaves of $T_{P}$ : $\Gamma[P]=\Sigma T_{P}$ and $P=\Gamma^{-1}\left[\Sigma T_{P}\right]$. For $t \in T_{P}, P_{t}:=\Gamma^{-1}\left[\Sigma T_{t}\right] \unlhd P$ is in $\mathcal{F}_{\lambda}^{I}$.

An ultrafilter $U$ over $I$ and a partition $P \in \mathcal{F}_{\Lambda}^{I}$ determine a TOU $\mathcal{U}_{P}$ over $T_{P}$ : If $P=\{0\}$, then $\mathcal{U}_{P}=0$, and if $P=\Sigma_{a \in P^{\alpha}} P_{a}$, then $\mathcal{U}_{P}(0)=U_{\alpha}$ and $\mathcal{U}_{P}(a \frown t)=$ $\mathcal{U}_{P_{a}}(t)$ otherwise.

Proposition 11.8 $\Sigma \mathcal{U}_{P}=\Gamma\left[U_{P}\right]$ and $U_{P}=\Gamma^{-1}\left[\Sigma \mathcal{U}_{P}\right]$. If $\widetilde{T} \preccurlyeq \mathcal{U}_{P} T_{P}$, then there is $\widetilde{P} \in \mathcal{F}_{\Lambda}^{I}, \widetilde{P} \unlhd P$, such that $\Sigma \widetilde{T} \subseteq \Gamma[\widetilde{P}], \Sigma \widetilde{T} \in \Sigma \mathcal{U}_{\widetilde{P}}, \widetilde{T} \equiv_{\mathcal{U}_{P}} T_{\widetilde{P}}$ and $P=\Sigma{ }_{s \in \widetilde{P}} P_{\Gamma(s)}$. 
Proof Straightforward induction on the rank of $P$.

For $\widetilde{T} \preccurlyeq \mathcal{U}_{P} T_{P}$ we define $P / \widetilde{T}:=\left\langle P_{t}: t \in \Sigma \widetilde{T}\right\rangle=\Sigma \mathcal{U}_{\tilde{P}}\left\langle P_{\Gamma(s)}: s \in \widetilde{P}\right\rangle$. We have $\mathcal{U} \ell\left(\mathbb{V} ; \Sigma \mathcal{U}_{\widetilde{P}}\right) \vDash " P / \widetilde{T} \in \mathcal{F}_{\mathfrak{k}(\Lambda)}^{\mathfrak{k}(I)} \wedge \mathcal{U}_{P} / \widetilde{T}=\mathcal{U}_{P / \widetilde{T}} "$. [Here $\mathfrak{k}=\mathfrak{k}_{\Sigma \mathcal{U}_{\tilde{P}}}$, and we identify $U$ and $\mathfrak{k}(U)$ in the last term.]

Proposition 11.9 Let $P, Q \in \mathcal{F}_{\Lambda}^{I}$ and $P \unlhd Q$. The projection $\pi_{P, Q}: U_{Q} \rightarrow U_{P}$ naturally induces a morphism of TOUs $\pi_{P, Q}: \mathcal{U}_{Q} \rightarrow \mathcal{U}_{P}$ so that the following diagram commutes.

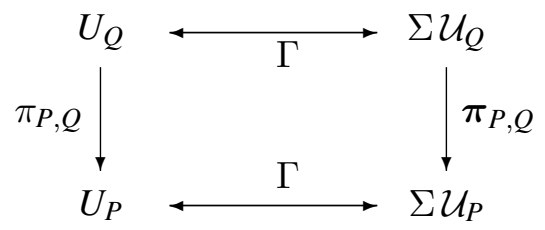

Proof By induction on the rank of $Q$. The case when either $P$ or $Q$ is $\{0\}$ is trivial, so let $P=\Sigma_{a \in P^{\alpha}} P_{a}, Q=\Sigma_{b \in P^{\beta}} Q_{b}$. As $P \unlhd Q$, we have to have $\beta \leq \alpha$.

Case 1: $\beta=\alpha$. This is a preserving case.

For each $a \in P^{\alpha}=Q^{\beta}$ we have $\pi_{P_{a}, Q_{a}}: \mathcal{U}_{Q_{a}} \rightarrow \mathcal{U}_{P_{a}}$. We define $\pi_{P, Q}(a)=a$ and $\pi_{P, Q}(a \frown t)=a \frown \pi_{P_{a}, Q_{a}}(t)$ for $t \in T_{Q_{a}}$. The verification of the claims is trivial.

Case 2: $\beta<\alpha$. This is a collapsing case.

It then follows from the definition of $\unlhd$ that $P \unlhd Q_{b}$ for all $b$, so we have $\pi_{P, Q_{b}}: \mathcal{U}_{Q_{b}} \rightarrow$ $\mathcal{U}_{P}$ for all $b \in Q^{\beta}$. We define $\pi_{P, Q}(b)=\{0\}$ and $\pi_{P, Q}(b \frown t)=\pi_{P, Q_{b}}(t)$ for $t \in T_{Q_{b}}$. The verification of the claims is again trivial.

It is also easy to see that $\pi_{P, R}=\pi_{P, Q} \circ \pi_{Q, R}$ if $P \unlhd Q \unlhd R$.

$\Gamma^{*}$ is an isomorphism of $\mathcal{U} \ell\left(\mathbb{V} ; \Sigma \mathcal{U}_{P}\right)$ and $\mathcal{U} \ell\left(\mathbb{V} ; U_{P}\right)$. In view of the preceding discussion, we henceforth informally identify these two interpretations. The isomorphism $\Gamma^{*}$ can be used to copy the stratification $\sqsubseteq_{\mathcal{U}_{P}}$ of $\mathcal{U} \ell\left(\mathbb{V} ; \Sigma \mathcal{U}_{P}\right)$ over to $\mathcal{U} \ell\left(\mathbb{V} ; U_{P}\right)$, and we may write simply $\mathcal{U} \ell t\left(\mathbb{V} ; U_{P}\right)$ in place of $\mathcal{U} \ell t\left(\mathbb{V} ; \Sigma \mathcal{U}_{P}\right)$.

From Propositions 11.6 and 11.9 it follows that $\left(\mathcal{F}_{\Lambda}^{I}, \unlhd\right), \mathbb{U}:=\left\langle\mathcal{U}_{P}: P \in \mathcal{F}_{\Lambda}^{I}\right\rangle$, $\phi:=\left\langle\left\{\varphi_{P, R}\right\}: P \unlhd Q\right\rangle$ specify a directed system of TOUs. The internally iterated ultrapower of the universe modulo $U$ along $\Lambda$ is the direct limit of this system, the stratified limit ultrapower $\mathcal{L U} \ell t(\mathbb{V} ; \mathbb{U}, \phi)$.

However, in order to construct an interpretation that satisfies SST ${ }^{\sharp}$, we need to internally iterate not a single ultrapower, but a limit ultrapower [such as the iterated ultrapower 
$\mathfrak{M}$ of the universe along $\left\langle U_{\delta}: \delta \in \bar{\Delta}\right\rangle$ constructed in Section 5 in order to obtain an interpretation for BST ]. Before describing this construction, we have to develop some further theory.

Let $I, J, \tau: J \rightarrow I$, and ultrafilters $U$ over $I, V$ over $J$ such that $U=\tau[V]$, be fixed. $\tau$ induces a mapping $\bar{\tau}: S e q_{\Lambda}^{J} \rightarrow \operatorname{Seq} q_{\Lambda}^{I}$ by $\bar{\tau}(s):=\tau \circ s$; we note that $\operatorname{dom} \bar{\tau}(s)=\operatorname{dom} s$. If $P \subseteq S e q_{\Lambda}^{I}$ is a partition, $\tau P:=\bar{\tau}^{-1}[P] \subseteq S e q_{\Lambda}^{J}$ is a partition, $\operatorname{dom} \tau P=\operatorname{dom} P$, and $\nu_{\tau P}=\nu_{P} \circ \bar{\tau}$. Let $\tau_{P}: \tau P \rightarrow P$ be defined as $\bar{\tau} \uparrow \tau P$.

It is easily seen that if $\left\langle P_{s}: s \in P\right\rangle$ is summable, then $\left\langle\tau\left(P_{\bar{\tau}(t)}\right): t \in \tau P\right\rangle$ is summable and $\tau\left(\Sigma_{s \in P} P_{s}\right)=\Sigma_{t \in \tau P} \tau\left(P_{\bar{\tau}(t)}\right)$.

If $P, Q \subseteq S e q_{\lambda}^{I}$ are partitions and $P \unlhd Q$, then $\tau P \unlhd \tau Q$ and the following diagram commutes.

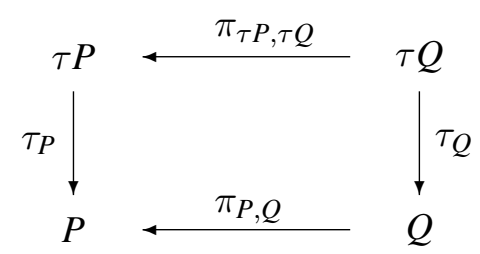

Proposition 11.10 If $P \in \mathcal{F}_{\lambda}^{I}$, then $\tau P \in \mathcal{F}_{\lambda}^{J}$, $\operatorname{rank} \tau P \leq \operatorname{rank} P, \tau_{P}: V_{\tau P} \rightarrow U_{P}$ is a morphism of ultrafilters, and $\tau_{P}$ naturally induces a morphism of TOUs $\tau_{P}: \mathcal{V}_{\tau P} \rightarrow \mathcal{U}_{P}$ so that the following diagram commutes.

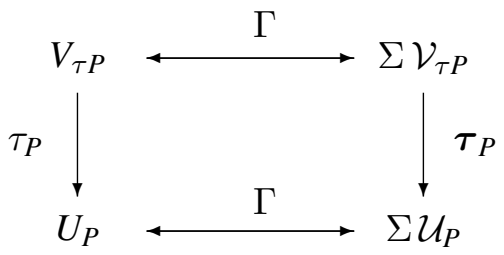

Also, for $P \unlhd Q \in \mathcal{F}_{\lambda}^{I}$, the following diagram commutes.

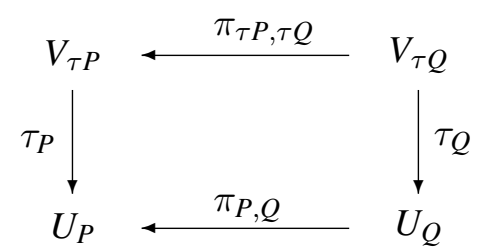

Proof By induction on $\operatorname{rank} P$. The nontrivial case is $P=\Sigma_{a \in P^{\alpha, I}} P_{a}$. We note that $\bar{\tau}(\langle(\alpha, i)\rangle)=\langle(\alpha, \tau(i))\rangle, \tau P^{\alpha, I}=P^{\alpha, J}$ and $\tau P=\Sigma_{b \in P^{\alpha, J}} \tau\left(P_{\bar{\tau}(b)}\right)$. As $\tau: V \rightarrow U$, clearly $\bar{\tau}\left\lceil P^{\alpha, J}: V_{\alpha} \rightarrow U_{\alpha}\right.$ and, by the inductive assumption, $\bar{\tau}\left\lceil\tau\left(P_{\bar{\tau}(b)}\right): V_{\tau\left(P_{\bar{\tau}(b)}\right)} \rightarrow\right.$ $U_{P_{\bar{\tau}(b)}}$. A straightforward computation shows that $\tau_{P}: V_{\tau P} \rightarrow U_{P}$. We omit the tedious verification of the remaining claims. 
Let now $(\mathbb{D}, \leq)$ be a directed preordering, $U_{d}$ an ultrafilter over $K_{d}$ for each $d \in \mathbb{D}$, and $\tau_{d, d^{\prime}}: U_{d^{\prime}} \rightarrow U_{d}$ a morphism such that $\tau_{d, d}=I d_{K_{d}}$ and $d \leq d^{\prime} \leq d^{\prime \prime} \Rightarrow \tau_{d, d^{\prime \prime}}=$ $\tau_{d, d^{\prime}} \circ \tau_{d^{\prime}, d^{\prime \prime}}$. [We are assuming that each $\phi_{d, d^{\prime}}$ had a unique representative $\tau_{d, d^{\prime}}$; this is done for simplicity, and is the case in the construction below of the interpretation for SST $^{\sharp}$. At the cost of further notational complications, the theory can easily be made to work for any directed system of ultrafilters.]

Definition 11.11 $\mathbb{D}_{\Lambda}:=\bigcup_{d \in \mathbb{D}}\{d\} \times \mathcal{F}_{\Lambda}^{K_{d}}$

$\langle d, P\rangle \leq\left\langle d^{\prime}, P^{\prime}\right\rangle$ iff $d \leq d^{\prime} \wedge \tau_{d, d^{\prime}} P \unlhd P^{\prime}$, for $\langle d, P\rangle,\left\langle d^{\prime}, P^{\prime}\right\rangle \in \mathbb{D}_{\Lambda}$

$\varphi_{d, P ; d^{\prime}, P^{\prime}}:=\left(\tau_{d, d^{\prime}}\right)_{P} \circ \pi_{\tau_{d, d^{\prime}} P, P^{\prime}}$

$U_{d, P}:=\left(U_{d}\right)_{P}$ is the ultrafilter over the partition $P$ associated with $U_{d}$.

It is easily seen that $\leq$ is a directed preordering of $\mathbb{D}_{\Lambda}$.

We point out in particular that

(a) $\langle d, P\rangle \leq\left\langle d^{\prime}, \tau_{d, d^{\prime}} P\right\rangle$ and $\varphi_{d, P ; d^{\prime}, \tau_{d, d^{\prime}} P}=\left(\tau_{d, d^{\prime}}\right)_{P}$, for $d \leq d^{\prime}, d, d^{\prime} \in \mathbb{D}$;

(b) $\langle d, P\rangle \leq\left\langle d, P^{\prime}\right\rangle$ and $\varphi_{d, P ; d^{\prime}, \tau_{d, d^{\prime}} P}=\pi_{P, P^{\prime}}$, for $P \unlhd P^{\prime}, P, P^{\prime} \in \mathcal{F}_{\Lambda}^{K_{d}}$.

Propositions 11.9 and 11.10 imply that $\varphi_{d, P ; d^{\prime}, P^{\prime}}:=\left(\boldsymbol{\tau}_{d, d^{\prime}}\right)_{P} \circ \boldsymbol{\pi}_{\tau_{d, d^{\prime}} P, P^{\prime}}: \mathcal{U}_{d^{\prime}, P^{\prime}} \rightarrow$ $\mathcal{U}_{d, P}$, that the diagram below commutes, that $\varphi_{d, P ; d, P}=I d_{P}$, and that $\varphi_{d, P ; d^{\prime \prime}, P^{\prime \prime}}=$ $\varphi_{d, P ; d^{\prime}, P^{\prime}} \circ \varphi_{d^{\prime}, P^{\prime} ; d^{\prime \prime}, P^{\prime \prime}}$ if $\langle d, P\rangle \leq\left\langle d^{\prime}, P^{\prime}\right\rangle \leq\left\langle d^{\prime \prime}, P^{\prime \prime}\right\rangle$, as well as boldface versions of these identities.

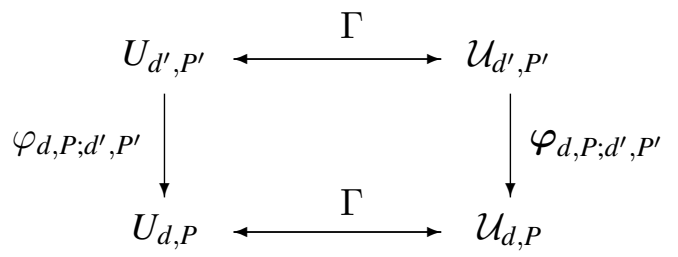

Thus $\left(\mathbb{D}_{\Lambda}, \leq\right), \mathbb{U}:=\left\langle\mathcal{U}_{d, P}:\langle d, P\rangle \in \mathbb{D}_{\Lambda}\right\rangle ; \phi:=\left\langle\left\{\varphi_{d, P ; d^{\prime}, P^{\prime}}\right\}:\langle d, P\rangle \leq\left\langle d^{\prime}, P^{\prime}\right\rangle\right\rangle$ specifies a directed system of TOUs. We call $\mathcal{L U} \ell t(\mathbb{V} ; \mathbb{U}, \phi)$ an internally iterated limit ultrapower of the universe along $\Lambda$ and $\left\langle U_{d}: d \in \mathbb{D}\right\rangle$. It is an interpretation for $\mathbf{S S T}^{b}$, of course.

\subsection{An interpretation for SST}

Finally, we fix a (nonempty) dense total ordering $\Lambda$ without endpoints [to be specific, the usual ordering of rational numbers], and let $\langle\bar{\Delta}, \leq\rangle$ and $\left\langle U_{\delta}: \delta \in \bar{\Delta}\right\rangle$ be as defined 
in Section 5. As in Section 5, $\overline{\mathbb{D}}=\mathcal{P}_{\text {fin }}(\bar{\Delta})$ is the class of all finite subsets of $\bar{\Delta}$, directed by inclusion $\subseteq$. To each $d=\left\{\delta_{1}, \ldots, \delta_{n}\right\} \in \overline{\mathbb{D}}$ where $\delta_{1}<\ldots<\delta_{n}$ we assign the ultrafilter $U_{d}:=U_{\delta_{1}} \otimes \ldots \otimes U_{\delta_{n}}$ over $K_{d}:=I_{\delta_{1}} \times \ldots \times I_{\delta_{n}} ; \quad U_{0}:=\{\{0\}\}$. For $d \subseteq d^{\prime}, \tau_{d, d^{\prime}}$ is the canonical projection of $K_{d^{\prime}}$ onto $K_{d}$, so $U_{d}=\tau_{d, d^{\prime}}\left[U_{d^{\prime}}\right]$.

The internally iterated limit ultrapower of the universe along $\Lambda$ and $\left\langle U_{d}: d \in \overline{\mathbb{D}}\right\rangle$ is denoted $\Im_{\Lambda}(\mathbb{V})=\left(\mathbb{V}^{*},=^{*}, \in^{*}, \sqsubseteq^{*}\right)$.

Our goal is to prove that $\Im_{\Lambda}(\mathbb{V})$ satisfies $\mathbf{S S T}^{\sharp}$. We need three technical lemmata.

Proposition 11.12 If $d=\left\{\delta_{1}<\ldots<\delta_{n}\right\}, U_{\delta_{1}}$ is $\kappa$-good, and $P \neq\{0\}$, then $U_{d, P}$ is $\kappa$-good.

Proof First, $P \neq\{0\}$ implies $P=\Sigma_{a \in P^{\alpha}} P_{a}$, and so $U_{d, P}=\left(U_{d}\right)_{P} \cong \Sigma_{U_{d}} U_{d, P_{\{\langle\alpha, i\rangle\}}}$ is $\kappa$-good, if $U_{d}$ is. Second, $U_{d}=U_{\delta_{1}} \otimes \ldots \otimes U_{\delta_{n}}$ is $\kappa$-good, if $U_{\delta_{1}}$ is.

Proposition 11.13 Let $\Lambda$ be a dense total ordering without endpoints. Let $\mathcal{V}$ be a TOU and $U$ an ultrafilter over $I$ such that $\mathcal{V}(t) \leq_{R K} U$ for all $t \in T_{\mathcal{V}} \backslash \Sigma T_{\mathcal{V}}$. Then there is $P \in \mathcal{F}_{\Lambda}^{I}$ with $\operatorname{rank} P=\operatorname{rank} \mathcal{V}$ and a morphism $\varphi: \mathcal{U}_{P} \rightarrow \mathcal{V}$. In particular, the assertion holds if $U$ is $\kappa$-good, with $\kappa>|\mathcal{V}(t)|$ for all $t$.

Proof By induction on rank of $\mathcal{V}$. If $\mathcal{V}=0$, all is clear.

Let $T_{\mathcal{V}}=\{0\} \cup \bigcup_{j \in J}\langle j\rangle \frown T_{\mathcal{V}_{j}}$. We fix $\alpha \in \Lambda$. The set $\Lambda(>\alpha)=\{\beta \in \Lambda: \alpha<\beta\}$ is dense with no endpoints. By the inductive assumption, there exist $P_{j} \in \mathcal{F}_{\Lambda(>\alpha)}^{I}$ with rank $P_{j}=\operatorname{rank} \mathcal{V}_{j}$ and morphisms $\varphi_{j}: \mathcal{U}_{P_{j}} \rightarrow \mathcal{V}_{j}$ for all $j \in J$. Let $f: I \rightarrow J$ be such that $\mathcal{V}(0)=f[U]$. We let $P:=\Sigma_{\{(\alpha, i)\} \in P^{\alpha}} P_{f(i)}$ and define $\varphi$ by $\varphi(0)=0$, $\varphi(\langle(\alpha, i)\rangle)=\langle f(i)\rangle, \varphi_{\langle i\rangle}=\varphi_{f(i)}$. It is easy to verify the required properties of $P$ and $\varphi$ [case (p) occurs].

Proposition 11.14 (Amalgamation Theorem) Let $\psi: \mathcal{W} \rightarrow \mathcal{V}$ be a morphism of TOUs. Let $\Lambda$ be a dense linear ordering without endpoints, $P \in \mathcal{F}_{\Lambda}^{I}$, and $\varphi: \mathcal{U}_{P} \rightarrow \mathcal{V}$ a morphism. Let $\widehat{U}$ be a $\kappa$-good ultrafilter over $\widehat{I}$, where $\kappa>|U|,|\mathcal{V}(t)|,|\mathcal{W}(t)|$ for all appropriate $t$, and $\tau: \widehat{I} \rightarrow I$ be such that $U=\tau[\widehat{U}]$. Then there is $\widehat{P} \in \mathcal{F}_{\Lambda}^{I}$ and a morphism $\widehat{\psi}: \widehat{\mathcal{U}}_{\hat{P}} \rightarrow \mathcal{W}$ such that $\tau P \unlhd \widehat{P}$ and, for $\widehat{\varphi}:=\tau_{P} \circ \pi_{\tau P, \hat{P}}, \quad \boldsymbol{\psi}(\widehat{\psi}(s))=$ $\varphi(\widehat{\varphi}(s))$ for $\Sigma \widehat{\mathcal{U}}_{\widehat{P}}$-almost all $s$; ie, the following diagram commutes $\Sigma \widehat{\mathcal{U}}_{\widehat{P}}$-almost everywhere.

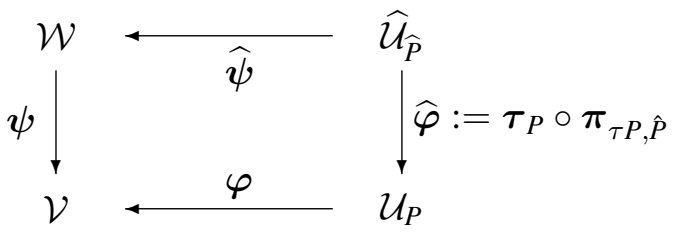


Proof By induction on rank of $\mathcal{W}$. If $\operatorname{rank} \mathcal{W}=0$, then $\mathcal{W}=\mathcal{V}=0$. We let $\widehat{P}:=\tau P$ [so $\left.\pi_{\tau P, \hat{P}}=I d_{\tau P}\right]$ and $\widehat{\psi}(t)=0$ for all $t \in T_{\hat{P}}$. From $\tau P: \widehat{\mathcal{U}}_{\tau P} \rightarrow \mathcal{U}_{P}$ the claims follow trivially.

If rank $\mathcal{W}>0$, then $T_{\mathcal{W}}=\{0\} \cup \bigcup_{k \in K}\langle k\rangle \frown T_{\mathcal{W}_{k}}$. We have to distinguish two cases.

Case (c): $\boldsymbol{\psi}$ is collapsing, ie, $\psi(\langle k\rangle)=0$ for all $k \in C:=[\operatorname{dom} \psi]_{0} \in \mathcal{W}(0)$.

We fix $\alpha \in \Lambda$ with $\alpha<\min P$.

For $k \in C$ then $\psi_{\langle k\rangle}: \mathcal{W}_{\langle k\rangle} \rightarrow \mathcal{V}$ and, by inductive assumption, there are $\widehat{P}_{k} \in \mathcal{F}_{\Lambda(>\alpha)}^{\hat{l}}$ and $\widehat{\boldsymbol{\psi}}_{k}: \widehat{\mathcal{U}}_{\hat{P}_{k}} \rightarrow \mathcal{W}_{\langle k\rangle}$ so that $\boldsymbol{\varphi} \circ \widehat{\boldsymbol{\varphi}}_{k}=\boldsymbol{\psi}_{\langle k\rangle} \circ \widehat{\boldsymbol{\psi}}_{k}\left[\right.$ for $\widehat{\boldsymbol{\varphi}}_{k}:=\boldsymbol{\tau}_{P} \circ \boldsymbol{\pi}_{\tau P, \hat{P}_{k}}$ ], ie, the diagram below commutes $\Sigma \widehat{\mathcal{U}}_{\widehat{P}_{k}}$-almost everywhere.

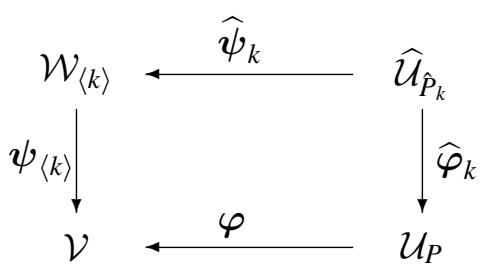

We fix $g: \widehat{I} \rightarrow K$ such that $g[\widehat{U}]=\mathcal{W}(0)$ and $\operatorname{ran} g \subseteq C$, and define $\widehat{P}:=$ $\Sigma_{\{(\alpha, \ell)\} \in \hat{P}^{\alpha}} \widehat{P}_{g(\ell)}$, and

$\widehat{\psi}(0)=0$

$\widehat{\psi}(\langle(\alpha, \ell)\rangle \frown t)=\langle g(\ell)\rangle \frown \widehat{\boldsymbol{\psi}}_{g(\ell)}(t)$ for $t \in T_{\hat{P}_{g(\ell)}}$.

Then $\boldsymbol{\psi}(\widehat{\boldsymbol{\psi}}(\langle(\alpha, \ell)\rangle \frown t))=\boldsymbol{\psi}\left(\langle g(\ell)\rangle \frown \widehat{\boldsymbol{\psi}}_{g(\ell)}(t)\right)=$ [because $\left.(\mathrm{c})\right]=\boldsymbol{\psi}_{\langle g(\ell)\rangle}\left(\widehat{\boldsymbol{\psi}}_{g(\ell)}(t)\right)=$ [inductive assumption] $=\varphi\left(\widehat{\varphi}_{g(\ell)}(t)\right)=\varphi(\widehat{\varphi}(\langle(\alpha, \ell)\rangle \frown t)) . \quad\left[\right.$ Note $\pi_{\tau P, \hat{P}_{g(\ell)}}(t)=$ $\left.\pi_{\tau P, \hat{P}}(\langle(\alpha, \ell)\rangle \frown t)\right]$.

Case (p): $\boldsymbol{\psi}$ is preserving, ie, $T_{\mathcal{V}}=\{0\} \cup \bigcup_{j \in J}\langle j\rangle \frown T_{\mathcal{V}_{j}}, \quad[\psi]_{0}: \subseteq K \rightarrow J$ and $\mathcal{V}(0)=[\boldsymbol{\psi}]_{0}[\mathcal{W}(0)], \quad \boldsymbol{\psi}_{\langle k\rangle}: \mathcal{W}_{\langle k\rangle} \rightarrow \mathcal{V}_{\boldsymbol{\psi}(\langle k\rangle)}$ for $k \in C=[\operatorname{dom} \boldsymbol{\psi}]_{0} \in \mathcal{W}(0)$.

Let $\widetilde{T}:=\varphi^{-1}(\{0\}) \preccurlyeq \mathcal{U}_{P} T_{P}$ and let $t \in \Sigma \widetilde{T}$. Then $[\varphi]_{t}: \subseteq\left[T_{P}\right]_{t} \rightarrow J, \mathcal{V}(0)=$ $[\varphi]_{t}[\mathcal{U}(t)]$, and $\varphi_{t \frown\langle a\rangle}: \mathcal{U}_{t \wedge\langle a\rangle} \rightarrow \mathcal{V}_{\left\langle[\varphi]_{t}(a)\right\rangle}$, where $[\varphi]_{t}(a) \in J$, for $a \in A_{t}:=$ $[\operatorname{dom} \varphi]_{t} \in \mathcal{U}(t)$. Note that $\left[T_{P}\right]_{t}=\{(\alpha, i): i \in I\}$ for some $\alpha \in \Lambda$ [dependent on $\left.t\right]$, and $\mathcal{U}(t)$ is $U_{\alpha}$, modulo the identification of $(\alpha, i)$ and $\{(\alpha, i)\}$.

By inductive assumption, for every pair $\langle a, k\rangle$ such that $\psi(\langle k\rangle)=\left\langle[\varphi]_{t}(a)\right\rangle=:\langle j\rangle \in J$, there exist $\widehat{P}_{t, a, k} \in \mathcal{F}_{\Lambda(>\alpha)}^{\hat{I}}$ and a morphism $\widehat{\psi}_{t, a, k}: \widehat{\mathcal{U}}_{\hat{P}_{t, a, k}} \rightarrow \mathcal{W}_{k}$ so that the following diagram commutes $\Sigma \widehat{\mathcal{U}}_{\hat{P}_{t, a, k}}$-almost everywhere, where $\widehat{\varphi}_{t, a, k}:=\boldsymbol{\tau}_{P_{t \sim\langle a\rangle}} \circ \pi_{\tau P_{t \frown\langle a\rangle}, \hat{P}_{t, a, k}}$. 


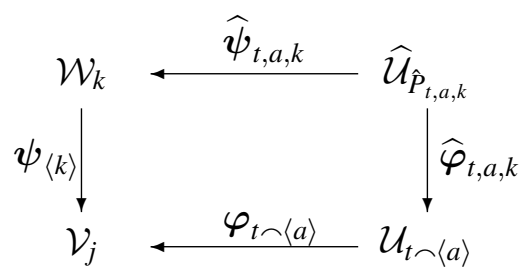

Letting $f_{t}=[\boldsymbol{\varphi}]_{t}, g_{t}=[\boldsymbol{\psi}]_{0}$, and $\widehat{f}_{t}(\ell)=(\alpha, \tau(\ell))$ for $\ell \in \widehat{I}$ [so that $\left.\mathcal{U}(t)=\widehat{f}_{t}[\widehat{U}]\right]$ and applying Proposition 3.13, we get $\widehat{g}_{t}: \subseteq \widehat{I} \rightarrow K$ so that $\widehat{g}_{t}[\widehat{U}]=\mathcal{W}(0)$ and $f_{t}\left(\widehat{f}_{t}(\ell)\right)=\widehat{U} g_{t}\left(\widehat{g}_{t}(\ell)\right)$ for $\ell \in D_{t} \in \widehat{U}$.

We define $\widehat{P}_{t}$ and $\widehat{\psi}_{t}: \widehat{\mathcal{U}}_{\widehat{P}_{t}} \rightarrow \mathcal{W}:$

$\widehat{P}_{t}:=\Sigma_{\{(\alpha, \ell)\} \in \hat{P}^{\alpha}} \widehat{P}_{t}^{\ell}$, where $\widehat{P}_{t}^{\ell}:=\widehat{P}_{t, \hat{f}_{t}(\ell), \hat{g}_{t}(\ell)}$ for $\ell \in D_{t}, \widehat{P}_{t}^{\ell}:=\tau P_{t \curvearrowleft\left\langle\hat{f}_{t}(\ell)\right\rangle}$ otherwise; $\widehat{\boldsymbol{\psi}}_{t}(\langle(\alpha, \ell)\rangle \frown s)=\left\langle\widehat{g}_{t}(\ell)\right\rangle \frown \widehat{\boldsymbol{\psi}}_{t, \hat{f}_{t}(\ell), \hat{g}_{t}(\ell)}(s)$, for $\ell \in D_{t} \in \widehat{U}$;

and show that the diagram

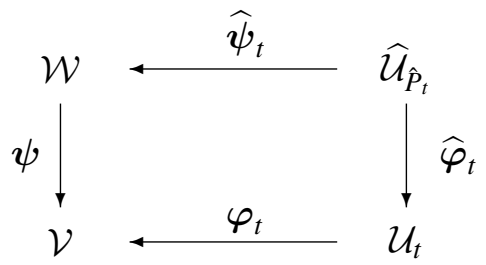

commutes $\Sigma \widehat{\mathcal{U}}_{\widehat{P}_{t}}$-almost everywhere, where $\widehat{\varphi}_{t}:=\tau_{P_{t}} \circ \pi_{\tau P_{t}, \hat{P}_{t}}$.

We note that $\min \widehat{P}_{t}=\alpha$ and $\widehat{\varphi}_{t}(\langle(\alpha, \ell)\rangle \frown s)=\left\langle\left(\alpha, \widehat{f}_{t}(\ell)\right)\right\rangle \frown \widehat{\varphi}_{t, \hat{f}_{t}(\ell), \hat{g}_{t}(\ell)}(s)$, and calculate:

$\boldsymbol{\varphi}_{t}\left(\widehat{\varphi}_{t}(\langle(\alpha, \ell)\rangle \frown s)\right)=\boldsymbol{\varphi}_{t}\left(\left\langle\left(\alpha, \widehat{f}_{t}(\ell)\right)\right\rangle \frown \widehat{\varphi}_{t, \hat{f}_{t}(\ell), \hat{g}_{t}(\ell)}(s)\right)=$ $\left\langle[\boldsymbol{\varphi}]_{t}\left(\widehat{f}_{t}(\ell)\right)\right\rangle \frown \boldsymbol{\varphi}_{t \frown\left\langle\widehat{f}_{t}(\ell)\right\rangle}\left(\widehat{\boldsymbol{\varphi}}_{t, \hat{f}_{t}(\ell), \hat{g}_{t}(\ell)}(s)\right)=\left\langle f_{t}\left(\widehat{f}_{t}(\ell)\right)\right\rangle \frown \boldsymbol{\varphi}_{t \frown\left\langle\widehat{f}_{t}(\ell)\right\rangle}\left(\widehat{\boldsymbol{\varphi}}_{t, \hat{f}_{t}(\ell), \hat{g}_{t}(\ell)}(s)\right)=$ $\left\langle g_{t}\left(\hat{g}_{t}(\ell)\right)\right\rangle \frown \boldsymbol{\psi}_{\left\langle\hat{g}_{t}(\ell)\right\rangle}\left(\widehat{\boldsymbol{\psi}}_{t, \hat{f}_{t}(\ell), \hat{g}_{t}(\ell)}(s)\right)=\left\langle[\psi]_{0}\left(\widehat{g}_{t}(\ell)\right)\right\rangle \frown \boldsymbol{\psi}_{\left\langle\hat{g}_{t}(\ell)\right\rangle}\left(\widehat{\boldsymbol{\psi}}_{t, \hat{f}_{t}(\ell), \hat{g}_{t}(\ell)}(s)\right)=$ $\boldsymbol{\psi}\left(\left\langle\widehat{g}_{t}(\ell)\right\rangle \frown \widehat{\boldsymbol{\psi}}_{t, \hat{f}_{t}(\ell), \hat{g}_{t}(\ell)}(s)\right)=\boldsymbol{\psi}\left(\boldsymbol{\psi}_{t}(\langle(\alpha, \ell)\rangle \frown s)\right)$,

for all $\ell \in D_{t}$ and all appropriate $s$.

Let $\widetilde{P} \in \mathcal{F}_{\Lambda}^{I}$ be such that $\Sigma \widetilde{T} \subseteq \widetilde{P}$ and $\Sigma \widetilde{T} \in \Sigma \mathcal{U}_{\widetilde{P}}$. We observe that $\tau_{\widetilde{P}}: \widehat{\mathcal{U}}_{\tau \widetilde{P}} \rightarrow \mathcal{U}_{\widetilde{P}}$, define $\widehat{T}:=\tau_{\widetilde{P}}^{-1}[\widetilde{T}] \subseteq \tau \widetilde{P}$, and note that $\Sigma \widehat{T} \in \Sigma \widehat{\mathcal{U}}_{\tau \widetilde{P}}$.

We finally define:

$\widehat{P}:=\Sigma_{s \in \tau \tilde{P}} \widehat{P}^{s}$, where $\widehat{P}^{s}=\widehat{P}_{\tau_{\tilde{P}}(s)}$ if $s \in \Sigma \widehat{T}, \widehat{P}^{s}=\tau P_{\tau_{\tilde{P}}(s)}$ otherwise $\widehat{\psi}(s)=0$ for $s \in \widehat{T}$ $\widehat{\psi}(s \frown t)=\widehat{\boldsymbol{\psi}}_{\tau_{\tilde{P}}(s)}(t)$ for $s \in \Sigma \widehat{T}$ and appropriate $t \in \widehat{P}_{\tau_{\tilde{P}}(s)}$. 
We omit the easy verification that $\widehat{\psi}$ is a morphism and check the commutativity. $\widehat{\varphi}(s)=\tau_{\tilde{P}}(s)$ for $s \in \widehat{T}$;

$\widehat{\varphi}(s \frown t)=\tau_{\tilde{P}}(s) \frown \widehat{\varphi}_{\tau_{\tilde{P}}(s)}(t)$ for $s \in \Sigma \widehat{T}$ and appropriate $t \in \widehat{P}_{\tau_{\tilde{P}}(s)}$.

For $s \in \widehat{T}, \varphi(\widehat{\varphi}(s))=\varphi\left(\tau_{\tilde{P}}(s)\right)=0$ because $\tau_{\tilde{P}}(s) \in \widetilde{T} ; \boldsymbol{\psi}(\widehat{\psi}(s))=\boldsymbol{\psi}(0)=0$ as well.

For $s \in \Sigma \widehat{T}$ and appropriate $t \in \widehat{P}_{\tau_{\tilde{P}}(s)}, \quad \varphi(\widehat{\varphi}(s \frown t))=\varphi\left(\tau_{\tilde{P}}(s) \frown \widehat{\varphi}_{\tau_{\tilde{P}}(s)}(t)\right)=$ $\varphi_{\tau_{\tilde{P}}(s)}\left(\widehat{\varphi}_{\tau_{\tilde{P}}(s)}(t)\right)=\psi\left(\widehat{\psi}_{\tau_{\tilde{P}}(s)}(t)\right)=\psi(\widehat{\psi}(s \frown t))$.

Main Theorem 1. $\Im_{\Lambda}(\mathbb{V})$ satisfies $\mathbf{S S T}^{\sharp}$.

Proof It remains to prove that $\Im_{\Lambda}(\mathbb{V}) \vDash(\forall \alpha)\left(\mathbf{F}_{\alpha}\right)$.

Claim. Let $\langle\widetilde{d}, \widetilde{P}\rangle \in \overline{\mathbb{D}}_{\Lambda}, f \in \mathbb{V} \widetilde{U}, U \in \boldsymbol{\beta}_{\infty} A, \quad V \in \boldsymbol{\beta}_{\infty}(A \times B)$, and $U=\bar{F}(V)$. If $\mathcal{U} \ell t(\mathbb{V} ; \widetilde{\mathcal{U}}) \vDash f \mathbf{M}_{0} \mathfrak{k}_{\Sigma \widetilde{\mathcal{U}}}(U), \widehat{d} \supseteq \widetilde{d}$, and $U_{\widehat{d}}$ is $\kappa$-good for sufficiently large $\kappa$, then there exist $\widehat{P}$ and $g \in \mathbb{V}^{U}$, such that $\langle\widetilde{d}, \widetilde{P}\rangle \leq\langle\widehat{d}, \widehat{P}\rangle \in \overline{\mathbb{D}}_{\Lambda}$ and $\mathcal{U} \ell t(\mathbb{V} ; \widehat{\mathcal{U}}) \vDash$ " $g \mathbf{M}_{0} \mathfrak{k}_{\Sigma \widehat{\mathcal{U}}}(V) \wedge \widehat{\varphi}^{*}(f)=\mathfrak{k}_{\Sigma \widehat{\mathcal{U}}}(F)(g)$ ", where $\widetilde{U}:=U_{\widetilde{d}, \widetilde{P}}, \quad \widetilde{\mathcal{U}}:=\mathcal{U}_{\tilde{d}, \widetilde{P}}, \quad \widehat{U}:=U_{\widehat{d}, \widehat{P}}$, $\widehat{\mathcal{U}}:=\mathcal{U}_{\widehat{d}, \widehat{P}}$, and $\widehat{\varphi}:=\varphi_{\widetilde{d}, \widetilde{P} ; \widehat{d}, \widehat{P}}$.

Proof of Claim. By Proposition 9.9, $F: B \rightarrow A$ induces a canonical morphism $\psi: \mathbf{V} \rightarrow \mathbf{U}$ of the TOUs associated with $U$ and $V$.

Let $\mathcal{U} \ell t(\mathbb{V} ; \widetilde{\mathcal{U}}) \vDash$ “ $\vec{u}$ is the pedigree for $f$ over $\mathfrak{k}_{\Sigma \widetilde{\mathcal{U}}}(A)$ "; note that then $\mathcal{U} \ell t(\mathbb{V} ; \widetilde{\mathcal{U}}) \vDash$ $u_{0}=\mathfrak{k}_{\Sigma \widetilde{\mathcal{U}}}(U)$. By Proposition 10.21, there is a morphism $\varphi: \widetilde{\mathcal{U}} \rightarrow \mathbf{U}$ such that $\mathcal{U} \ell t(\mathbb{V} ; \widetilde{\mathcal{U}}) \vDash " \varphi^{*}\left(\vec{d}_{U}\right)=\vec{u}^{+} \wedge \varphi^{*}\left(e_{U}\right)=f$ ". We fix $\widehat{d} \in \mathbb{D}$ so that $\widetilde{d} \subseteq \widehat{d}$ and $U_{\hat{d}}$ is $\kappa$-good, where $\kappa$ is sufficiently large for the Amalgamation Lemma to apply. There is then $\widehat{P}$ and $\widehat{\psi}: \widehat{\mathcal{U}}_{\hat{P}} \rightarrow \mathbf{V}$ so that $\langle\widetilde{d}, \widetilde{P}\rangle \leq\langle\widehat{d}, \widehat{P}\rangle$ and the following diagram commutes $\Sigma \widehat{\mathcal{U}}$-almost everywhere $\left[\widehat{\mathcal{U}}:=\widehat{\mathcal{U}}_{\hat{P}}\right.$ and $\left.\widehat{\varphi}:=\varphi_{\tilde{d}, \tilde{P} ; \hat{d}, \hat{P}}\right]$.

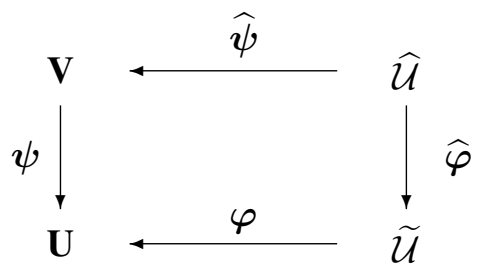


If we write $\vec{d}_{U}(t)=t$ and $e_{U}(t)=t_{n}$ for $t=\left\langle t_{0}, \ldots, t_{n}\right\rangle \in \Sigma T_{\mathbf{U}}$ [or $e_{U}(t)=U$ if $t=0]$ as in Proposition 10.20 and apply $\varphi^{*}$ to its assertion, we have $\mathcal{U} \ell t(\mathbb{V} ; \widetilde{\mathcal{U}}) \vDash$ “ $\left\langle\mathfrak{k}_{\Sigma \tilde{\mathcal{U}}}(U)\right\rangle \frown \varphi^{*}\left(\vec{d}_{U}\right)$ is the pedigree for $\varphi^{*}\left(e_{U}\right)$ over $\mathfrak{k}_{\Sigma \widetilde{\mathcal{U}}}(A)$ ". Hence $\mathcal{U} \ell t(\mathbb{V} ; \widehat{\mathcal{U}}) \vDash$ “ $\left\langle\mathfrak{k}_{\Sigma \widehat{\mathcal{U}}}(U)\right\rangle \frown \widehat{\varphi}^{*}\left(\varphi^{*}\left(\vec{d}_{U}\right)\right)$ is the pedigree for $\widehat{\varphi}^{*}(f)$ over $\mathfrak{k}_{\Sigma \widehat{\mathcal{U}}}(A)$ ". In particular, $\mathcal{U} \ell t(\mathbb{V} ; \widehat{\mathcal{U}}) \vDash \widehat{\varphi}^{*}(f) \mathbf{M}_{0} \mathfrak{k}_{\Sigma \widehat{\mathcal{U}}}(U)$.

By Proposition 10.20 we have $\mathcal{U} \ell t(\mathbb{V} ; \mathbf{V}) \vDash "\left\langle\mathfrak{k}_{\Sigma} \mathbf{V}(V)\right\rangle \frown \vec{d}_{V}$ is the pedigree for $e_{V}$ over $\mathfrak{k}_{\Sigma \mathbf{V}}(B)$ ", so $\mathcal{U} \ell t(\mathbb{V} ; \widehat{\mathcal{U}}) \vDash “\left\langle\mathfrak{k}_{\Sigma} \widehat{\mathcal{U}}^{*}(V)\right\rangle \frown \widehat{\boldsymbol{\psi}}^{*}\left(\vec{d}_{V}\right)$ is the pedigree for $\widehat{\boldsymbol{\psi}}^{*}\left(e_{V}\right)$ over $\mathfrak{k}_{\Sigma \widehat{\mathcal{U}}^{(B)}}$ ". In particular, $\mathcal{U} \ell t(\mathbb{V} ; \widehat{\mathcal{U}}) \vDash \widehat{\boldsymbol{\psi}}^{*}\left(e_{V}\right) \mathbf{M}_{0} \mathfrak{k}_{\Sigma \widehat{\mathcal{U}}^{(}}(V)$.

Subclaim. $\mathcal{U} \ell t(\mathbb{V} ; \mathbf{V}) \vDash \boldsymbol{\psi}^{*}\left(e_{U}\right)=\mathfrak{k}_{\Sigma \mathbf{V}}(F)\left(e_{V}\right)$.

Proof of Subclaim. Assume $U \notin \boldsymbol{\beta}_{0} A, \quad V \notin \boldsymbol{\beta}_{0} B$. We recall [Proposition 10.20] that $e_{V}\left(\left\langle v_{0}, \ldots, v_{n}\right\rangle\right)=v_{n}, e_{U}\left(\left\langle u_{0}, \ldots, u_{k}\right\rangle\right)=u_{k}$, and, as $\boldsymbol{\psi}$ is the canonical morphism induced by $F, \psi\left(\left\langle v_{0}, \ldots, v_{n}\right\rangle\right)=\left\langle\ldots, \bar{F}\left(v_{n}\right)\right\rangle \Sigma \mathbf{V}$-almost everywhere, and $\bar{F}\left(v_{n}\right)=F\left(e_{V}\left(\left\langle v_{0}, \ldots, v_{n}\right\rangle\right)\right)$ [see the proof of Proposition 9.9 and Definition 9.3]. Hence $\psi^{*}\left(e_{U}\right)\left(\left\langle v_{0}, \ldots, v_{n}\right\rangle\right)=e_{U}\left(\psi\left(\left\langle v_{0}, \ldots, v_{n}\right\rangle\right)\right)=e_{U}\left(\left\langle\ldots, \bar{F}\left(v_{n}\right)\right\rangle\right)$ and $\bar{F}\left(v_{n}\right)=$ $\boldsymbol{\psi}^{*}\left(e_{U}\right)\left(\left\langle v_{0}, \ldots, v_{n}\right\rangle\right) \Sigma \mathbf{V}$-almost everywhere.

It follows that $\boldsymbol{\psi}^{*}\left(e_{U}\right)\left(\left\langle v_{0}, \ldots, v_{n}\right\rangle\right)=F\left(e_{V}\left(\left\langle v_{0}, \ldots, v_{n}\right\rangle\right)\right) \Sigma \mathbf{V}$-almost everywhere, ie, $\mathcal{U} \ell t(\mathbb{V} ; \mathbf{V}) \vDash \boldsymbol{\psi}^{*}\left(e_{U}\right)=\mathfrak{k}_{\Sigma} \mathbf{V}(F)\left(e_{V}\right)$. The cases when $V \in \boldsymbol{\beta}_{0} B$ or $U \in \boldsymbol{\beta}_{0} A$ are similar.

From the Subclaim we get $\mathcal{U} \ell t(\mathbb{V} ; \widehat{\mathcal{U}}) \vDash \widehat{\psi}^{*}\left(\psi^{*}\left(e_{U}\right)\right)=\mathfrak{k}_{\Sigma \widehat{\mathcal{U}}}(F)\left(\widehat{\psi}^{*}\left(e_{V}\right)\right)$; but also $\widehat{\boldsymbol{\psi}}^{*}\left(\boldsymbol{\psi}^{*}\left(e_{U}\right)\right)=e_{U}(\boldsymbol{\psi} \circ \widehat{\boldsymbol{\psi}})=_{\Sigma \hat{\mathcal{U}}} e_{U}(\varphi \circ \widehat{\varphi})=\widehat{\varphi}^{*}\left(\varphi^{*}\left(e_{U}\right)\right)={ }_{\Sigma \hat{\mathcal{U}}} \widehat{\varphi}^{*}(f)$. We let $g:=\widehat{\boldsymbol{\psi}}^{*}\left(e_{V}\right)$ and conclude that $\mathcal{U} \ell t(\mathbb{V} ; \widehat{\mathcal{U}}) \vDash \widehat{\varphi}^{*}(f)=\mathfrak{k}_{\Sigma \widehat{\mathcal{U}}^{(}}(F)(g) \wedge g \mathbf{M}_{0} \mathfrak{k}_{\Sigma \widehat{\mathcal{U}}}(V)$.

The Claim immediately implies that $\Im_{\Lambda}(\mathbb{V})$ satisfies $\mathbf{F}_{0}$ :

If $\Im_{\Lambda}(\mathbb{V}) \vDash f \mathbf{M}_{0} \mathfrak{k}(U)$, we fix $\langle\widetilde{d}, \widetilde{P}\rangle \in \overline{\mathbb{D}}_{\Lambda}$ so that $f \in \mathbb{V}^{\Sigma \tilde{\mathcal{U}}}$. Then also $\mathcal{U} \ell t(\mathbb{V} ; \tilde{\mathcal{U}}) \vDash$

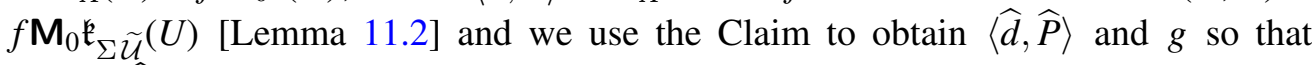
$\mathcal{U} \ell t(\mathbb{V} ; \widehat{\mathcal{U}}) \vDash " \widehat{\varphi}^{*}(f)=\mathfrak{k}_{\Sigma \widehat{\mathcal{U}}}(F)(g) \wedge g \mathbf{M}_{0} \mathfrak{k}_{\Sigma \widehat{\mathcal{U}}}(V) "$.

From this, $\Im_{\Lambda}(\mathbb{V}) \vDash " f=\mathfrak{k}(F)(g) \wedge g \mathbf{M}_{0} \mathfrak{k}(V)$ " follows, again by Lemma 11.2.

Before proceeding, we restate the Claim:

If $\langle\widetilde{d}, \widetilde{P}\rangle \in \overline{\mathbb{D}}_{\Lambda}$ and $\mathcal{U} \ell t\left(\mathbb{V} ; \mathcal{U}_{\tilde{d}, \widetilde{P}}\right) \vDash " u, v, a, b, F \sqsubseteq 0 \wedge u \in \boldsymbol{\beta}_{\infty} a \wedge v \in \boldsymbol{\beta}_{\infty} b \wedge u=$ $\bar{F}(v) \wedge f \mathbf{M}_{0} u$ ", then there exist $\widehat{d}, \widehat{P}$ and $g \in \mathbb{V} \widehat{P}$ such that $\langle\widetilde{d}, \widetilde{P}\rangle \leq\langle\widehat{d}, \widehat{P}\rangle \in \overline{\mathbb{D}}_{\Lambda}$ and $\mathcal{U} \ell t\left(\mathbb{V} ; \mathcal{U}_{\widehat{d}, \widehat{P}}\right) \vDash "$ " $\widehat{\varphi}^{*}(f)=\widehat{\varphi}^{*}(F)(g) \wedge g \mathbf{M}_{0} \widehat{\varphi}^{*}(v)$ ", where $\widehat{\varphi}:=\varphi_{\tilde{d}, \widetilde{P}} ; \widehat{d}, \widehat{P}$. [Note that $\widehat{\varphi}^{*}(v)=\mathfrak{k}_{\Sigma} \hat{\mathcal{U}}(V)$ for $v=\mathfrak{k}_{\Sigma \widetilde{U}}(V)$, and similarly for $F$; also, without loss of generality $\operatorname{dom} g=\widehat{P}$.] 
We use the Factoring Theorem to prove that $\Im_{\Lambda}(\mathbb{V})$ satisfies $\mathbf{F}_{\alpha}$ for arbitrary level $\alpha$. Let $\Im_{\Lambda}(\mathbb{V}) \vDash " f \mathbf{M}_{\alpha} U \wedge U \in \boldsymbol{\beta}_{\infty} A \wedge V \in \boldsymbol{\beta}_{\infty} B \wedge U=\bar{F}(V) \wedge A, B, U, V, F \sqsubseteq \alpha$ ". We fix $\langle d, P\rangle \in \overline{\mathbb{D}}_{\Lambda}$ so that $f, \alpha, A, B, U, V, F \in \mathbb{V}^{\Sigma \mathcal{U}_{P}}$; the preceding statement is then satisfied in $\mathcal{U} \ell t\left(\mathbb{V} ; \mathcal{U}_{P}\right)$. [By Theorem 10.7, $\mathcal{U} \ell t\left(\mathbb{V} ; \mathcal{U}_{P}\right) \vDash f \mathbf{M}_{\alpha} U^{\prime}$ for some $U^{\prime} \in \boldsymbol{\beta}_{\infty} A$, so $\Im_{\Lambda}(\mathbb{V}) \vDash f \mathbf{M}_{\alpha} U^{\prime}$ by Lemma 11.2, and $U={ }^{*} U^{\prime}$ by Proposition 10.3.] By Proposition 7.2 and Corollary 7.3(a) applied to $\alpha$, there is a tree $T(\alpha) \preccurlyeq \mathcal{U}_{P} T_{P}$ such that $h \sqsubseteq \mathcal{U}_{P} \alpha \Leftrightarrow T(h) \preccurlyeq \mathcal{U}_{P} T(\alpha)$.

By Proposition 11.8 and subsequent discussion, there is a $\widetilde{P} \in \mathcal{F}_{\Lambda}^{K_{d}}, \widetilde{P} \unlhd P$, such that $\Sigma T(\alpha) \subseteq \Gamma[\widetilde{P}], \quad \Sigma T(\alpha) \in \Sigma \mathcal{U}_{\widetilde{P}}$, and $\widetilde{T}:=T_{\widetilde{P}} \equiv_{\mathcal{U}_{P}} T(\alpha)$. Evidently, $\mathcal{U} \ell t\left(\mathbb{V} ; \mathcal{U}_{P}\right.$ $T(\alpha))$ is isomorphic to $\mathcal{U} \ell t\left(\mathbb{V} ; \mathcal{U}_{\widetilde{P}}\right)$. By the Factoring Theorem, we see that the ultrapower $\left(\mathcal{U} \ell\left(\mathbb{V} ; \Sigma \mathcal{U}_{P}\right), \sqsubseteq \mathcal{U}_{P} ; D_{\widetilde{T}}\right)$ is isomorphic to $\left[\mathcal{U} \ell t\left(\mathbb{V} ; \mathcal{U}_{P} / \widetilde{T}\right)\right]^{\mathcal{U} \ell\left(\mathbb{V} ; \Sigma \mathcal{U}_{\tilde{P}}\right)}$ via the isomorphism $\Omega_{\widetilde{T}, \mathcal{U}}$ defined by $\Omega_{\widetilde{T}, \mathcal{U}}(g)=g / \widetilde{T}$.

From $\mathcal{U} \ell t\left(\mathbb{V} ; \Sigma \mathcal{U}_{P}\right) \vDash f \mathbf{M}_{\alpha} U$ we get $\left(\mathcal{U} \ell\left(\mathbb{V} ; \Sigma \mathcal{U}_{P}\right)\right.$, $\left.\complement_{\mathcal{U}_{P} ; D_{\widetilde{T}}}\right) \vDash f \mathbf{M}_{0} U$. Applying the isomorphism $\Omega$ and writing $\mathfrak{k}$ for $\mathfrak{k}_{\Sigma \mathcal{U}_{\tilde{P}}}$,

$\mathcal{U} \ell\left(\mathbb{V} ; \Sigma \mathcal{U}_{\widetilde{P}}\right) \vDash “\langle k(d), P / \widetilde{T}\rangle \in \overline{\mathbb{D}}_{\mathfrak{k}(\Lambda)\left(>\nu_{\tilde{P}}\right)} \wedge \mathcal{U} \ell t\left(\mathbb{V} ; \mathcal{U}_{P} / \widetilde{T}\right) \vDash[U / \widetilde{T}, V / \widetilde{T}, A / \widetilde{T}, B / \widetilde{T}$, $\left.F / \widetilde{T} \sqsubseteq 0 \wedge U / \widetilde{T} \in \boldsymbol{\beta}_{\infty}(A / \widetilde{T}) \wedge V / \widetilde{T} \in \boldsymbol{\beta}_{\infty}(B / \widetilde{T}) \wedge U / \widetilde{T}=\overline{F / \widetilde{T}}(V / \widetilde{T}) \wedge f / \widetilde{T} \mathrm{M}_{0} U / \widetilde{T}\right] "$. $\mathcal{U} \ell\left(\mathbb{V} ; \Sigma \mathcal{U}_{\widetilde{P}}\right)$ satisfies $\mathbf{Z F C}$ and therefore the restated Claim. Hence $\mathcal{U} \ell\left(\mathbb{V} ; \Sigma \mathcal{U}_{\widetilde{P}}\right) \vDash$ $\left(\exists d^{\prime}, q, g^{\prime}\right)\left[\left\langle d^{\prime}, q\right\rangle \in \overline{\mathbb{D}}_{\mathfrak{k}(\Lambda)\left(>\nu_{\tilde{P}}\right)} \wedge\langle\mathfrak{k}(d), P / \widetilde{T}\rangle \leq\left\langle d^{\prime}, q\right\rangle \wedge \operatorname{dom} g^{\prime}=q \wedge \mathcal{U} \ell t\left(\mathbb{V} ; \mathcal{U}_{q}\right) \vDash\right.$ “ $\left.\varphi_{\mathfrak{k}(d), P / \widetilde{T} ; d^{\prime}, q}^{*}(f / \widetilde{T})=\varphi_{\mathfrak{k}(d), P / \widetilde{T} ; d^{\prime}, q}^{*}(F / \widetilde{T})\left(g^{\prime}\right) \wedge g^{\prime} \mathbf{M}_{0} \varphi_{\mathfrak{k}(d), P / \widetilde{T} ; d^{\prime}, q}^{*}(V / \widetilde{T}) ”\right]$.

We observe that, without loss of generality, $d^{\prime}=\Sigma \mathcal{U}_{\tilde{p}} \mathfrak{k}(\widehat{d})$ for $\widehat{d} \supseteq d$. This is true because there is $\widehat{d} \supseteq d$ such that $\mathcal{U} \ell\left(\mathbb{V} ; \Sigma \mathcal{U}_{\widetilde{P}}\right) \vDash$ “k $(\widehat{d})$ is $\mathfrak{k}(\kappa)$-good", for arbitrarily large $\kappa$. We write $\tau$ for $\tau_{d, \widehat{d}}$ and apply the morphism $\tau_{\widetilde{P}}$ to obtain [writing now $\mathfrak{k}$ for

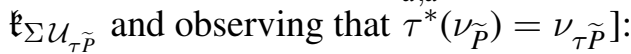

$$
\begin{gathered}
\mathcal{U} \ell\left(\mathbb{V} ; \Sigma \mathcal{U}_{\tau \widetilde{P}}\right) \vDash\left(\exists q, g^{\prime}\right)\left[\langle\mathfrak{k}(\widehat{d}), q\rangle \in \overline{\mathbb{D}}_{\mathfrak{k}(\Lambda)\left(>\nu_{\tau \widetilde{P}}\right)} \wedge\left\langle\mathfrak{k}(d), \tau^{*}(P / \widetilde{T})\right\rangle \leq\langle\mathfrak{k}(\widehat{d}), q\rangle \wedge\right. \\
\operatorname{dom} g^{\prime}=q \wedge \mathcal{U} \ell t\left(\mathbb{V} ; \Sigma \mathcal{U}_{q}\right) \vDash “ \varphi_{\mathfrak{k}(d), \tau^{*}(P / \widetilde{T}) ; \mathfrak{k}(\widehat{d}), q^{*}}^{*}\left(\tau^{*}(f / \widetilde{T})\right)= \\
\left.\varphi_{\mathfrak{k}(d), \tau^{*}(P / \widetilde{T}) ; \mathfrak{k}(\widehat{d}), q}^{*}\left(\tau^{*}(F / \widetilde{T})\right)\left(g^{\prime}\right) \wedge g^{\prime} \mathbf{M}_{0} \boldsymbol{\varphi}_{\mathfrak{k}(d), \tau^{*}(P / \widetilde{T}) ; \mathfrak{k}(\widehat{d}), q}^{*}\left(\tau^{*}(V / \widetilde{T})\right) "\right]
\end{gathered}
$$

Claim. $q=\Sigma_{\mathcal{U}_{\tau \widetilde{P}}} \widehat{P} / \tau \widetilde{T}$, where $\tau_{d, \hat{d}} P \unlhd \widehat{P} \in \mathcal{F}_{\Lambda}^{K_{\widehat{d}}}$.

\section{Proof of Claim.}

For almost all $t \in \tau \widetilde{P}$ we have $\left\langle d, \tau^{*}(P / \widetilde{T})(t)\right\rangle \leq\langle\widehat{d}, q(t)\rangle$ and $q(t) \in \mathcal{F}_{\Lambda\left(>\nu_{\tau}(t)\right)}^{K_{\widehat{P}}}$. Modifying $q$ if necessary, we can assume this is true for all $t \in \tau \widetilde{P}$. As $\tau^{*}(P / \widetilde{T})(t)=$ $(P / \widetilde{T})(\tau(t))=P_{\tau(t)}$, we have $\left\langle d, P_{\tau(t)}\right\rangle \leq\langle\widehat{d}, q(t)\rangle$, which means $\tau P_{\tau(t)} \unlhd q(t)$. We can thus define $\widehat{P}:=\Sigma_{t \in \tau \widetilde{P}} q(t)$. As $P=\Sigma_{s \in \widetilde{P}} P_{s}, \tau P=\Sigma_{t \in \tau \widetilde{P}} \tau P_{\tau(t)} \unlhd \widehat{P}$. 
Hence $\langle d, P\rangle \leq\langle\widehat{d}, \widehat{P}\rangle$; we let $\varphi:=\varphi_{d, P ; \widehat{d}, \widehat{P}}$. Also, $g^{\prime}=\Sigma \mathcal{U}_{\tilde{P}} g / \tau \widetilde{P}$ for some $g \in \mathbb{V} \widehat{P}$.

Claim. $\mathcal{U} \ell\left(\mathbb{V} ; \Sigma \mathcal{U}_{\tau \widetilde{P}}\right) \vDash \varphi_{\mathfrak{k}(d), \tau^{*}(P / \widetilde{T}) ; \mathfrak{k}(\widehat{d}), q}=\varphi / \tau \widetilde{P}$.

Proof of Claim. For $\Sigma \mathcal{U}_{\tau \widetilde{P}}$-almost all $t$ we have $\varphi_{d, \tau^{*}(P / \widetilde{T})(t) ; d^{\prime}, q(t)}(t)=\varphi_{d, P_{\tau(t)} ; d^{\prime}, \widehat{P}_{t}}$ $=\bar{\tau} \circ \pi_{P_{\tau(t)}, \widehat{P}_{t}}$. For all $s \in \widehat{P}_{t}$ now $\varphi(t) \frown \varphi_{t}(s)=\varphi(t \frown s)=\bar{\tau}\left(\pi_{\tau P, \widehat{P}}(t \frown s)\right)=$ $\bar{\tau}\left(t \frown \pi_{\tau P, \widehat{P}}(t \frown s)\right)=\bar{\tau}(t) \frown \bar{\tau}\left(\pi_{P_{\tau(t)}, \widehat{P}_{t}}(s)\right)=\varphi(t) \frown \varphi_{d, P_{\tau(t)} ; d^{\prime}, \widehat{P}_{t}}(s)$. We conclude that $(\varphi / \tau \widetilde{P})(t)=\varphi_{t}=\varphi_{d, P_{\tau(t)} ; d^{\prime}, \widehat{P}_{t}}=\varphi_{d, \tau^{*}(P / \widetilde{T})(t) ; d^{\prime}, q(t)}(t)$.

We now have

$\mathcal{U} \ell\left(\mathbb{V} ; \Sigma \mathcal{U}_{\tau \widetilde{P}}\right) \vDash “ \mathcal{U} \ell t\left(\mathbb{V} ; \mathcal{U}_{\widehat{P} / \tau \widetilde{T}}\right) \vDash\left[(\varphi / \tau \widetilde{P})^{*}\left(\tau^{*}(f / \widetilde{T})\right)=(\varphi / \tau \widetilde{P})^{*}\left(\tau^{*}(F / \widetilde{T})\right)(g / \tau \widetilde{T})\right.$ $\left.\wedge(g / \tau \widetilde{T}) \mathbf{M}_{0}(\varphi / \tau \widetilde{P})^{*}\left(\tau^{*}(V / \widetilde{T})\right)\right] "$

We use the Factoring Theorem for Morphisms $7.12(* * *)\left[\right.$ let $\mathcal{U}^{1}=\mathcal{U}_{P}, \widetilde{\mathcal{U}}^{1}=\mathcal{U}_{\tilde{P}}$, $\mathcal{U}^{2}=\mathcal{U}_{\widehat{P}}, \quad \widetilde{\mathcal{U}}^{2}=\mathcal{U}_{\tau \widetilde{P}}, \quad \mathcal{U}^{2} / \widetilde{T}^{2}=\mathcal{U}_{\widehat{P}} / \tau \widetilde{P}, \varphi=\varphi_{d, P, \widehat{d}, \widehat{P}}, \quad \widehat{\varphi}=\varphi\left\lceil\tau \widetilde{P}=\tau_{P}\right]$ to conclude that $\mathcal{U} \ell\left(\mathbb{V} ; \Sigma \mathcal{U}_{\tau \widetilde{P}}\right) \vDash " \mathcal{U} \ell t\left(\mathbb{V} ; \mathcal{U}_{\widehat{P} / \tau \widetilde{T}}\right) \vDash\left[\varphi^{*}(f) / \tau \widetilde{P}=\left(\varphi^{*}(F) / \tau \widetilde{P}\right)(g / \tau \widetilde{T}) \wedge\right.$ $\left.(g / \tau \widetilde{T}) \mathbf{M}_{0}\left(\varphi^{*}(V) / \tau \widetilde{P}\right)\right]$ ". By the Factoring Theorem this means that, finally,

$\left(\mathcal{U} \ell\left(\mathbb{V} ; \Sigma \mathcal{U}_{\widehat{P}}\right), \sqsubseteq \mathcal{U}_{\widehat{P}} ; D_{\tau \widetilde{T}}\right) \vDash “ \varphi^{*}(f)=\varphi^{*}(F)(g) \wedge g \mathbf{M}_{0} \varphi^{*}(V)$ ", ie, $\mathcal{U} \ell t\left(\mathbb{V} ; \mathcal{U}_{\widehat{P}}\right) \vDash$ " $\varphi^{*}(f)=\varphi^{*}(F)(g) \wedge g \mathrm{M}_{\varphi^{*}(\alpha)} \varphi^{*}(V)$ ".

By Lemma 11.2, $\Im_{\Lambda}(\mathbb{V}) \vDash " f=F(g) \wedge g \mathbf{M}_{\alpha} V "$.

We consider one additional auxiliary axiom.

\section{Block Idealization:}

For all $0 \sqsubset \beta, A, B \sqsubseteq 0$, and $R \sqsubseteq \beta$ such that $R \subseteq A \times B$,

$$
\begin{gathered}
\left(\forall a \in \mathcal{P}^{\mathrm{fin}} A\right)[a \sqsubset \beta \Rightarrow(\exists y)(\forall x \in a)(\langle x, y\rangle \in R)] \Leftrightarrow \\
(\exists y \sqsubseteq \beta)(\forall x \in A)(x \sqsubset \beta \Rightarrow\langle x, y\rangle \in R) .
\end{gathered}
$$

Theorem 11.15 $\Im_{\Lambda}(\mathbb{V})$ satisfies Block Idealization.

Proof Assume that $\Im_{\Lambda}(\mathbb{V}) \vDash " R \subseteq A^{*} \times B^{*} \wedge 0 \sqsubset \beta \wedge R \sqsubseteq \beta \wedge\left(\forall a \in \mathcal{P}^{\text {fin }}\left(A^{*}\right)\right)$ $[a \sqsubset \beta \Rightarrow(\exists y)(\forall x \in a)(\langle x, y\rangle \in R)]$ ". We fix $\langle d, P\rangle \in \overline{\mathbb{D}}_{\Lambda}$ so that $R, \beta \in \mathbb{V}^{U}$, where $U:=U_{d, P}$ and $U_{\delta_{1}}$ [hence, $\left.U_{d}\right]$ is $\kappa-\operatorname{good}\left[d=\left\{\delta_{1}<\ldots<\delta_{n}\right\}\right.$ ] for fixed $\kappa>|A|$. By Proposition 11.8 there is $\widetilde{P} \unlhd P$ such that $T(\beta) \equiv_{U} T_{\widetilde{P}}$; we let $\widetilde{U}:=U_{d, \widetilde{P}}$ and fix $\gamma$ such that $T(\gamma)^{+} \equiv_{U} T(\beta)$. Then $R \in \mathbb{V}^{\widetilde{U}}, y \in \mathbb{V}^{\widetilde{U}} \Rightarrow y \sqsubseteq^{*} \beta$, and $\mathcal{U} \ell t(\mathbb{V} ; \widetilde{U}) \vDash$ “ $\left(\forall a \in \mathcal{P}^{\text {fin }}\left(\mathfrak{k}_{\widetilde{U}}(A)\right)\right)[a \sqsubseteq \gamma \Rightarrow(\exists y)(\forall x \in a)(\langle x, y\rangle \in R)]$ ”. As $\gamma \sqsubset^{*} \beta$ and each $U(t) \cong U_{d}$ is $\kappa$-good, it follows by Proposition 8.1 that $\mathcal{U} \ell(\mathbb{V} ; \widetilde{U}) \vDash(\kappa$ Idealization $)^{\mathbb{S}_{\gamma}}$. Hence there is $y \in \mathbb{V} \widetilde{U}$ such that $\mathcal{U} \ell t(\mathbb{V} ; \widetilde{U}) \vDash "\left(\forall x \in \mathfrak{k}_{U}(A)\right)(x \sqsubset$ $\beta \Rightarrow\langle x, y\rangle \in R)$ " and so $\mathcal{U} \ell t(\mathbb{V} ; U) \vDash " y \sqsubseteq \beta \wedge\left(\forall x \in \mathfrak{k}_{\widetilde{U}}(A)\right)(x \sqsubset \beta \Rightarrow\langle x, y\rangle \in R)$ ". By Proposition 10.16 finally $\Im_{\Lambda}(\mathbb{V}) \vDash " y \sqsubseteq \beta \wedge\left(\forall x \in A^{*}\right)\left(x \sqsubset \beta \Rightarrow\langle x, y\rangle \in R^{*}\right)$ ". 


\section{GRIST.}

In this section we prove the equivalence of SST $^{\sharp}$ with the axiomatic system GRIST formulated in the Introduction, derive some of its basic metamathematical properties, and give a number of consequences that are useful for development of mathematics in GRIST.

\subsection{Metamathematics of SST ${ }^{\sharp}$.}

First we draw some model-theoretic consequences of Main Theorem 1. Most of the proofs are either obvious or entirely analogous to those given in Section 5, and are omitted.

Corollary 12.1 SST $^{\sharp}$ has a realization in $\mathbf{Z F C}$.

Corollary 12.2 SST $^{\sharp}$ is a conservative extension of $\mathbf{Z F C}$. In particular, SST ${ }^{\sharp}$ is consistent relative to $\mathbf{Z F C}$.

Corollary 12.3 Every model $\mathbf{M}$ of $\mathbf{Z F C}$ has an extension to a model $\mathbf{N}$ of $\mathbf{S S T}^{\sharp}$ with $\mathbf{N} \uparrow \mathbb{S}_{0}^{\mathbf{N}}=\mathbf{M}$.

Corollary 12.4 If $\mathbf{N}_{1}$ and $\mathbf{N}_{2}$ are models of $\mathbf{S S T} \mathbf{S T}^{\sharp}$, with $\mathbf{N}_{1} \uparrow \mathbb{S}_{0}^{\mathbf{N}_{1}}=\mathbf{N}_{2} \uparrow \mathbb{S}_{0}^{\mathbf{N}_{2}}=\mathbf{M}$, then they are $\mathcal{L}_{\infty, \omega}$-elementarily equivalent, where $\mathcal{L}$ is the $\in$ - - -language with a name for each $x \in \mathbf{M}$. If also $\left|\mathbf{N}_{1}\right|=\left|\mathbf{N}_{2}\right|=\aleph_{0}$, then $\mathbf{N}_{1}$ and $\mathbf{N}_{2}$ are isomorphic, by an isomorphism which is identity on $\mathbf{M}$.

Corollary 12.5 Every countable model of ZFC has a unique (up to isomorphism) extension to a countable model of SST $^{\sharp}$.

Corollary 12.6 If $\mathbf{N}_{1}$ is a model of $\mathbf{S S T}^{b}$ and $\mathbf{N}_{2}$ is a model of $\mathbf{S S T} \mathbf{T}^{\sharp}$, with $\mathbf{N}_{1}\left\lceil\mathbb{S}_{0}^{\mathbf{N}_{1}}=\right.$ $\mathbf{N}_{2}\left\lceil\mathbb{S}_{0}^{\mathbf{N}_{2}}=\mathbf{M}\right.$ and $\left|\mathbf{N}_{1}\right|=\left|\mathbf{N}_{2}\right|=\aleph_{0}$, then there is an $\in$-elementary embedding of $\mathbf{N}_{1}$ into $\mathbf{N}_{2}$ which is the identity on $\mathbf{M}$

In view of Theorem 11.1, this result implies that every stratified limit ultrapower of the universe, constructed internally in $\mathbf{M} \vDash \mathbf{Z F C}$, $\mathbf{M}$ countable, has an $\in$-elementary embedding into the unique extension of $\mathbf{M}$ to a countable model of SST ${ }^{\sharp}$. In this sense, SST $\sharp$ is the theory of the "universal" stratified limit ultrapower. 
Corollary 12.7 (Completeness over ZFC) If $\mathbf{T} \supseteq \mathbf{Z F C}$ is a complete consistent theory (in the $\in$-language), then $\mathbf{T}+\mathbf{S S T}^{\sharp}$ is a complete consistent theory (in the $\in-\sqsubseteq$-language).

Corollary 12.8 Let $\mathcal{P}$ be a statement in the $\in-\sqsubseteq$-language.

If $\mathbf{Z F C} \vdash$ “ $\Im_{\Lambda}(\mathbb{V}) \vDash \mathcal{P}$ ”, then $\mathbf{S S T}^{\sharp} \vdash \mathcal{P}$.

Proof Assuming the contrary, SST $\sharp \wedge \neg \mathcal{P}$ has a countable model $\mathbf{N}$ and $\mathbf{M}:=\mathbf{N}$ $\mathbb{S}_{0}^{\mathbf{N}} \vDash \mathbf{Z F C}$. By Corollary $12.4, \mathbf{N}$ has to be isomorphic to $\mathbf{N}^{\prime}:=\Im_{\Lambda}(\mathbb{V})^{\mathbf{M}}$, obtained by constructing $\Im_{\Lambda}(\mathbb{V})$ inside $\mathbf{M}$; but $\mathbf{N}^{\prime} \vDash \mathcal{P}$.

\section{Corollary 12.9 SST SS $^{\sharp}$ Block Idealization.}

We give this indirect argument via Theorem 11.15 in order to avoid technicalities attendant on a direct proof of this fact.

Corollary 12.10 SST $^{\sharp}$ is finitely axiomatizable over $\mathbf{Z F C}$.

Proof Proposition 1.2 shows that Transfer follows from a finite number of its instances. This result (see also [3]) shows that SST is finitely axiomatizable over ZFC. We get SST $^{\sharp}$ by adding the axioms $(\forall \alpha)\left(\mathbf{B}_{\alpha}\right)$ and $(\forall \alpha)\left(\mathbf{F}_{\alpha}\right)$.

The following Normal Form Theorem, an analog of Theorem 5.4 for BST, is a fundamental metamathematical result about SST ${ }^{\sharp}$.

Theorem 12.11 (SST $\left.{ }^{\sharp}\right)$ (Normal Form Theorem) Let $\mathcal{P}\left(x_{1}, \ldots, x_{k}\right)$ be any $\in-\sqsubseteq-$ formula. There is an $\in$-formula $\mathcal{Q}(U)$ (obtained effectively from $\mathcal{P}$ ) such that, for all $\alpha$ and all $\left\langle x_{1}, \ldots, x_{k}\right\rangle \mathbf{M}_{\alpha} U, \quad \mathcal{P}^{\alpha}\left(x_{1}, \ldots, x_{k}\right) \Leftrightarrow \mathcal{Q}(U)$.

In particular, $\mathcal{P}^{\alpha}\left(x_{1}, \ldots, x_{k}\right) \Leftrightarrow$ $(\exists U)\left(\left\langle x_{1}, \ldots, x_{k}\right\rangle \mathbf{M}_{\alpha} U \wedge \mathcal{Q}(U)\right) \Leftrightarrow(\forall U)\left(\left\langle x_{1}, \ldots, x_{k}\right\rangle \mathbf{M}_{\alpha} U \Rightarrow \mathcal{Q}(U)\right)$.

Proof We proceed by induction on the complexity of $\mathcal{P}$.

Let $\vec{u}$ be an $\alpha$-pedigree for $x=\left\langle x_{1}, \ldots, x_{k}\right\rangle$ with $u_{0}=U$. The function $E_{\mathbf{U}}$ is defined in Proposition 10.20. In the isomorphism $\mathfrak{j}_{\alpha}:[\mathcal{U} \ell t(\mathbb{V} ; \mathbf{U})]^{\mathbb{S}_{\alpha}} \rightarrow \mathbb{S}_{\alpha}\left[\vec{u}^{+}\right]$, $\mathfrak{j}_{\alpha}\left(E_{\mathbf{U}}\right)=E_{\mathbf{U}}\left(\vec{u}^{+}\right)=x=\left\langle x_{1}, \ldots, x_{k}\right\rangle$ and $j_{\alpha}\left(\pi_{i} \circ E_{\mathbf{U}}\right)=x_{i}$ for $i=1, \ldots, k$.

We have

$x_{i} \in x_{j} \Leftrightarrow \pi_{i} \circ E_{\mathbf{U}} \in_{\Sigma \mathbf{U}} \pi_{j} \circ E_{\mathbf{U}} \Leftrightarrow\left\{t \in \Sigma T_{\mathbf{U}}: \pi_{i}\left(E_{\mathbf{U}}(t)\right) \in \pi_{j}\left(E_{\mathbf{U}}(t)\right)\right\} \in \Sigma \mathbf{U} ;$ 
$x_{i} \sqsubseteq_{\alpha} x_{j} \Leftrightarrow \pi_{i} \circ E_{\mathbf{U}} \sqsubseteq_{\Sigma \mathbf{U}} \pi_{j} \circ E_{\mathbf{U}} \Leftrightarrow\left(\forall T^{\prime} \preccurlyeq \Sigma \mathbf{U} T_{\mathbf{U}}\right)\left(\pi_{j} \circ E_{\mathbf{U}} \in \mathbb{V}^{\Sigma \mathbf{U}^{\prime}} \Rightarrow \pi_{i} \circ E_{\mathbf{U}} \in\right.$ $\mathbb{V}^{\Sigma \mathbf{U}^{\prime}}$ ), where $\mathbf{U}^{\prime}:=\mathbf{U} \uparrow T^{\prime}$.

The induction step is clear for $\wedge$ and $\neg$.

We now consider $\mathcal{P}\left(x_{1}, \ldots, x_{k}\right)$ of the form $(\exists y) \mathcal{P}_{1}\left(x_{1}, \ldots, x_{k}, y\right)$. By the inductive assumption, there is $\mathcal{Q}_{1}$ such that, if $\left\langle x_{1}, \ldots, x_{k}, y\right\rangle \mathbf{M}_{\alpha} V$, then

$$
\mathcal{P}_{1}^{\alpha}\left(x_{1}, \ldots, x_{k}, y\right) \Leftrightarrow \mathcal{Q}_{1}(V) .
$$

Let $\mathcal{Q}(U)$ be the formula $(\exists V)\left(\overline{\pi_{1, \ldots, k}}(V)=U \wedge \mathcal{Q}_{1}(V)\right)$.

If $\mathcal{P}^{\alpha}\left(x_{1}, \ldots, x_{k}\right)$ holds, fix $y$ such that $\mathcal{P}_{1}^{\alpha}\left(x_{1}, \ldots, x_{k}, y\right)$ holds. By $\mathbf{B}_{\alpha}$ there exists $V \in \mathbb{S}_{\alpha}$ such that $U=\overline{\pi_{1, \ldots, k}}(V)$ and $\left\langle x_{1}, \ldots, x_{k}, y\right\rangle \mathbf{M}_{\alpha} V$. From (*) we get $\mathcal{Q}_{1}(V)$. Hence $\mathcal{Q}(U)$ holds.

Conversely, if $\mathcal{Q}(U)$ holds, then [by Transfer into $\mathbb{S}_{\alpha}$ ] there is $V \in \mathbb{S}_{\alpha}$ such that $\overline{\pi_{1, \ldots, k}}(V)=U$ and $\mathcal{Q}_{1}(V)$. By $\mathbf{F}_{\alpha}$ there exists $y$ with $\left\langle x_{1}, \ldots, x_{k}, y\right\rangle \mathbf{M}_{\alpha} V$. Hence $\mathcal{P}_{1}^{\alpha}\left(x_{1}, \ldots, x_{k}, y\right)$ holds, and $\mathcal{P}^{\alpha}\left(x_{1}, \ldots, x_{k}\right)$ holds.

Corollary 12.12 If $\left\langle x_{1}, \ldots, x_{k}\right\rangle$ and $\left\langle x_{1}^{\prime}, \ldots, x_{k}^{\prime}\right\rangle$ have the same $\alpha$-type, then they are $\alpha$-indiscernible, ie, $\mathcal{P}^{\alpha}\left(x_{1}, \ldots, x_{k}\right) \Leftrightarrow \mathcal{P}^{\alpha}\left(x_{1}^{\prime}, \ldots, x_{k}^{\prime}\right)$ for all $\in$ - - -formulas $\mathcal{P}$.

Corollary 12.13 (Boldface Normal Form Theorem) Let $\mathcal{P}\left(a, x_{1}, \ldots, x_{k}\right)$ be any $\in$ $\sqsubseteq$-formula. There is an $\in$-formula $\mathcal{Q}(a, U)$ (obtained effectively from $\mathcal{P}$ ) such that, for all $\alpha$ and all $U$ with $\left\langle x_{1}, \ldots, x_{k}\right\rangle \mathbf{M}_{\alpha} U$,

$$
\left(\forall a \in \mathbb{S}_{\alpha}\right)\left(\mathcal{P}^{\alpha}\left(a, x_{1}, \ldots, x_{k}\right) \Leftrightarrow \mathcal{Q}(a, U)\right)
$$

Proof Let $\mathcal{P}^{\prime}(a, x)$ be the formula $\left(\exists z_{1}, \ldots, z_{k}\right)\left(x=\left\langle z_{1}, \ldots, z_{k}\right\rangle \wedge \mathcal{P}\left(a, z_{1}, \ldots, z_{k}\right)\right)$ and $\mathcal{Q}^{\prime}(V)$ the $\in$-formula corresponding to $\mathcal{P}^{\prime}(a, x)$ by the Normal Form Theorem. By Proposition 9.7, for every $a \in \mathbb{S}_{\alpha}, A:=\{a\}$, and $U \in \boldsymbol{\beta}_{\infty} B$, there is a unique $U_{a} \in \boldsymbol{\beta}_{\infty}(A \times B)$ such that $\overline{\pi_{1}}\left(U_{a}\right)=a$ and $\overline{\pi_{2}}\left(U_{a}\right)=U$. Hence $\left\langle x_{1}, \ldots, x_{k}\right\rangle \mathbf{M}_{\alpha} U \Leftrightarrow$ $\left\langle a,\left\langle x_{1}, \ldots, x_{k}\right\rangle\right\rangle \mathbf{M}_{\alpha} U_{a}$. We let $\mathcal{Q}(a, U)$ be the formula $\mathcal{Q}^{\prime}\left(U_{a}\right)$.

\subsection{Equivalence of GRIST and SST ${ }^{\sharp}$.}

We need one further technical result.

Definition 12.14 (ZFC) We fix $V \in \boldsymbol{\beta}_{1} B \backslash \boldsymbol{\beta}_{0} B$ and define an ultrafilter $U^{\sim} V \in$ $\boldsymbol{\beta}_{\xi}(A \times B)$, for each $U \in \boldsymbol{\beta}_{\xi} A \backslash \boldsymbol{\beta}_{0} A$, by recursion. 
If $U \in \boldsymbol{\beta}_{1} A \backslash \boldsymbol{\beta}_{0} A, \quad U^{\sim} V:=U \otimes V$. We remark that $U \otimes V$ is used only for convenience; one could employ instead any $W$ such that $\pi_{1}[W]=U$ and $\pi_{2}[W]=V$.

At stage $\xi>1$ we assume that the one-one mapping $\sigma_{<\xi}: U^{\prime} \mapsto\left(U^{\prime}\right)^{\sim} V$ of $\boldsymbol{\beta}_{<\xi} A \backslash$ $\boldsymbol{\beta}_{0} A$ to $\boldsymbol{\beta}_{<\xi}(A \times B)$ has been defined, $\operatorname{rank}\left(U^{\prime}\right)^{\sim} V=\operatorname{rank} U^{\prime}, \overline{\pi_{1}}\left(\left(U^{\prime}\right)^{\sim} V\right)=U^{\prime}$, and $\overline{\pi_{2}}\left(\left(U^{\prime}\right)^{\sim} V\right)=V$ for all such $U^{\prime}$. For $U \in \boldsymbol{\beta}_{\xi} A \backslash \boldsymbol{\beta}_{<\xi} A$, the ultrafilter $\sigma_{<\xi}[U]$ is then nonprincipal and does not contain $\boldsymbol{\beta}_{<\eta}(A \times B)$ for any $\eta<\xi$. Hence $\sigma_{<\xi}[U] \in$ $\boldsymbol{\beta}_{\xi}(A \times B) \backslash \boldsymbol{\beta}_{<\xi}(A \times B)$, and we let $U^{\sim} V:=\sigma_{<\xi}[U]$. We observe that $\pi_{1}\left[U^{\sim} V\right]=U$ and $\overline{\pi_{2}}\left[U^{\sim} V\right]=W_{V, \boldsymbol{\beta}_{<\xi}(A \times B)}$; hence $\overline{\pi_{1}}\left(U^{\sim} V\right)=U$ and $\overline{\pi_{2}}\left(U^{\sim} V\right)=V$.

Proposition 12.15 (SST $\left.{ }^{\sharp}\right) \quad$ Let $B, V \in \mathbb{S}_{\alpha}, V \in \boldsymbol{\beta}_{1} B \backslash \boldsymbol{\beta}_{0} B$. For every $\beta \sqsupset \alpha$ there is $z \boxminus \beta$ such that $z \mathbf{M}_{\alpha} V$.

Proof Fix $x \boxminus \beta, x \in A \in \mathbb{S}_{\alpha}$, and an $\alpha$-pedigree $\vec{u}=\left\langle u_{0}, \ldots, u_{\nu}\right\rangle$ for $x$ over $A$ with $U:=u_{0}$, so $x \mathbf{M}_{\alpha} U$. As $\beta \sqsupset \alpha, \nu>0$ and $U \notin \boldsymbol{\beta}_{0} A$. $\mathbf{F}_{\alpha}$ implies that there exists $z$ such that $\langle x, z\rangle \mathbf{M}_{\alpha} U^{\sim} V$. As $\overline{\pi_{2}}\left(U^{\sim} V\right)=V, z \mathbf{M}_{\alpha} V$ [Proposition 10.14, Theorem 10.10]. It remains to prove that $z \boxminus x$.

Let $\vec{v}=\left\langle v_{0}, \ldots, v_{\mu}\right\rangle$ be an $\alpha$-pedigree for $\langle x, z\rangle$ over $A \times B$; in particular, $v_{0}=U^{\sim} V$. Let $\gamma \boxminus v_{\mu-1} ;\left\langle v_{\mu-1}, v_{\mu}\right\rangle$ is a $\gamma$-pedigree for $\langle x, z\rangle$ over $A \times B$.

Claim 1. Each $v_{m}, m<\mu$, is of the form $\left(U^{\prime}\right)^{\sim} V$ for some $U^{\prime} \in \boldsymbol{\beta}_{\infty} A \backslash \boldsymbol{\beta}_{0} A$.

\section{Proof of Claim 1.}

Consider the largest $m<\mu$ for which $v_{m}=\left(U^{\prime}\right)^{\sim} V$ for some $U^{\prime}$. Then either $\operatorname{rank} U^{\prime}>1$ and $v_{m+1} \in\left\{\left(U^{\prime \prime}\right)^{\sim} V: U^{\prime \prime} \in \operatorname{dom} U^{\prime}\right\} \in\left(U^{\prime}\right)^{\sim} V$, and we have a contradiction, or rank $U^{\prime}=1$, so $v_{m+1} \in \boldsymbol{\beta}_{0} A$ and $m+1=\mu$.

Hence $v_{\mu-1}=\left(U^{\prime}\right)^{\sim} V$. It follows that $\gamma \sqsubset z, x$; otherwise, either $U^{\prime}$ or $V$ would be principal. The conclusion follows from:

Claim 2. If $\gamma \sqsubset \bar{x} \sqsubset \bar{y}$, then every $\gamma$-pedigree for $\langle\bar{x}, \bar{y}\rangle$ has length at least 3 .

Proof of Claim 2.

Assume that $\left\langle v_{0}, v_{1}\right\rangle$ is a $\gamma$-pedigree for $\langle\bar{x}, \bar{y}\rangle$ of length 2 . Then $\left\langle\overline{\pi_{2}}\left(v_{0}\right), \overline{\pi_{2}}\left(v_{1}\right)\right\rangle$ is a $\gamma$-pedigree for $\bar{y}$, so $v_{1} \sqsupseteq \bar{y}$; also, $\left\langle\overline{\pi_{1}}\left(v_{0}\right), \overline{\pi_{1}}\left(v_{1}\right)\right\rangle$ is a $\gamma$-pedigree for $\bar{x}$. We note in particular that $\overline{\pi_{1}}\left(v_{0}\right), \overline{\pi_{2}}\left(v_{0}\right)$ are both nonprincipal. Let $\delta \boxminus \bar{x}$; then $v_{0} \sqsubset \delta \sqsubset v_{1}$ and $\left\langle v_{0}, v_{1}\right\rangle$ is also a $\delta$-pedigree for $\langle\bar{x}, \bar{y}\rangle$. By Corollary 10.14 [Theorem 10.10] it follows that $\left\{\overline{\pi_{1}}\left(v_{0}\right), \overline{\pi_{1}}\left(v_{1}\right)\right\}$ is the range of a $\delta$-pedigree for $\bar{x}$, a contradiction, because $\bar{x} \in \mathbb{S}_{\delta}$ while $\overline{\pi_{1}}\left(v_{0}\right)$ is nonprincipal. 
Main Theorem 2. The theories GRIST and $\mathbf{S S T}^{\sharp}$ are equivalent.

\section{Proof that SST $^{\sharp} \vdash$ GRIST:}

\section{Relativization.}

Let $x, A, V \in \mathbb{S}_{\alpha}, V \in \boldsymbol{\beta}_{1} A \backslash \boldsymbol{\beta}_{0} A$. By $\mathbf{F}_{\alpha}$, there exists $y \mathbf{M}_{\alpha} V$; then $x \sqsubset y$.

To prove density, let $x \sqsubset y$ be given. We let $\alpha \boxminus x, \beta \boxminus y$, and fix $A, U, B, V \in \mathbb{S}_{\alpha}$ such that $V \in \boldsymbol{\beta}_{1} B \backslash \boldsymbol{\beta}_{0} B, U \in \boldsymbol{\beta}_{2} A \backslash \boldsymbol{\beta}_{1} A$ and $z \boxminus \beta, z \mathbf{M}_{\alpha} V$. By $\mathbf{F}_{\alpha}$, there exists $w$ such that $\langle w, z\rangle \mathbf{M}_{\alpha} U^{\sim} V$; the $\alpha$-pedigree for $\langle w, z\rangle$ is $\left\langle u_{0}, u_{1}, u_{2}\right\rangle$ [because rank $U^{\sim} V=2$ ] and $\alpha \sqsubset u_{1} \sqsubset u_{2}$. The proof of 12.15 establishes that $w \boxminus z$, hence $u_{2}=\langle w, z\rangle \boxminus \beta$ and $x \sqsubset u_{1} \sqsubset y$.

\section{Transfer.}

Let $\alpha \sqsubseteq \beta$ and $x_{1}, \ldots, x_{k} \sqsubseteq \alpha$. We fix $A \in \mathbb{S}_{\alpha}$ such that $x:=\left\langle x_{1}, \ldots, x_{k}\right\rangle \in A$ and note that $x \mathbf{M}_{\beta} W_{x, A}$ for all $\beta \sqsupseteq \alpha$. By the Normal Form Theorem, $\mathcal{P}^{\alpha}\left(x_{1}, \ldots, x_{k}\right)$ $\Leftrightarrow \mathcal{Q}\left(W_{x, A}\right) \Leftrightarrow \mathcal{P}^{\beta}\left(x_{1}, \ldots, x_{k}\right)$.

\section{Granularity.}

The Normal Form Theorem provides a formula $\mathcal{Q}(U)$ such that $\left\langle x_{1}, \ldots, x_{k}\right\rangle \mathbf{M}_{\alpha} U$ implies $\mathcal{P}^{\alpha}\left(x_{1}, \ldots, x_{k}\right) \Leftrightarrow \mathcal{Q}(U)$. Let $\left\langle u_{i}: i \leq \nu\right\rangle$ be the 0 -pedigree for $\left\langle x_{1}, \ldots, x_{k}\right\rangle$. By Proposition 10.5, for $u_{i} \sqsubseteq \alpha \sqsubset u_{i+1},\left\langle x_{1}, \ldots, x_{k}\right\rangle \mathbf{M}_{\alpha} u_{i}$, and for $u_{\nu} \sqsubseteq \alpha$, $\left\langle x_{1}, \ldots, x_{k}\right\rangle \mathbf{M}_{\alpha} u_{\nu}$. If $\mathcal{P}^{\alpha}\left(x_{1}, \ldots, x_{k}\right)$ holds for some $\alpha$, then $\mathcal{Q}\left(u_{i}\right)$ holds for some $i$, and there is a least $i$ for which $\mathcal{Q}\left(u_{i}\right)$ holds. Then $\alpha \boxminus u_{i}$ for this $i$ has the required properties.

\section{Idealization.}

We prove the implication from left to right; the converse is an immediate consequence of the fact that SST $\vdash$ " $a \in \mathcal{P}^{\mathrm{fin}} A \cap \mathbb{S}_{\alpha} \Rightarrow a \subseteq A \cap \mathbb{S}_{\alpha}$ " [cf. 3.11].

Let $\mathcal{P}^{\prime}(x, z)$ be the formula $(\exists A, \bar{x}, y)(z=\langle\langle A, \bar{x}\rangle, y,\rangle \wedge \mathcal{P}(x, A, \bar{x}, y))$ and let $\mathcal{Q}(W)$ be the formula corresponding to $\mathcal{P}^{\prime}(x, z)$ by the Normal Form Theorem.

Let $\left\langle u_{n}: n \leq \nu\right\rangle$ be an $\alpha$-pedigree for $\langle A, \bar{x}\rangle$, and $U:=u_{n}$ where $u_{n} \sqsubseteq \beta \sqsubset u_{n+1}$ [ $U:=u_{\nu}$, if $\left.u_{\nu} \sqsubseteq \beta\right]$; then $\langle A, \bar{x}\rangle \mathbf{M}_{\beta} U$. Given $a \in \mathcal{P}^{\text {fin }} A, a \sqsubset \beta$, there is $y$ such that $(\forall x \in a) \mathcal{P}^{\beta}(x, A, \bar{x}, y)$; let $V$ be such that $\langle\langle A, \bar{x}\rangle, y,\rangle \mathbf{M}_{\beta} V$ and $U=\overline{\pi_{1}}(V)$. For all $x \in a, \quad\langle x,\langle\langle A, \bar{x}\rangle, y\rangle,\rangle \mathbf{M}_{\beta} V_{x}$, hence $\mathcal{Q}\left(V_{x}\right)$. So the following $\in$-statement holds:

$$
\left(\forall a \in \mathcal{P}^{\mathrm{fin}} A\right)\left[a \sqsubset \beta \Rightarrow(\exists V)(\forall x \in a)\left(U=\overline{\pi_{1}}(V) \wedge \mathcal{Q}\left(V_{x}\right)\right)\right] .
$$


By Collection (in $\mathbb{V}$ ) and Boundedness, there is a set $B \in \mathbb{S}_{\alpha}$ such that $(\exists V$ ) can be replaced by $(\exists V \in B)$. We now use Block Idealization, transferred from level 0 to level $\alpha$, [define $R(x, V)$ by $(\forall x \in a)\left(U=\overline{\pi_{1}}(V) \wedge \mathcal{Q}\left(V_{x}\right)\right)$ and note $R \sqsubseteq \beta$ ] to conclude that

$$
\left(\exists V \in B \cap \mathbb{S}_{\beta}\right)(\forall x \in A)\left[x \sqsubset \beta \Rightarrow\left(U=\overline{\pi_{1}}(V) \wedge \mathcal{Q}\left(V_{x}\right)\right)\right] .
$$

Fix such $V$ and use $\mathbf{F}_{\beta}$ to get $y$ such that $\langle\langle A, \bar{x}\rangle, y\rangle \mathbf{M}_{\beta} V$. Then for all $x \sqsubseteq \beta$ we have $\langle x,\langle\langle A, \bar{x}\rangle, y\rangle,\rangle \mathbf{M}_{\beta} V_{x}$. If $x \sqsubset \beta$ and $x \in A$, then also $\mathcal{Q}\left(V_{x}\right)$ holds; hence, $\mathcal{P}^{\beta}(x, A, \bar{x}, y)$ holds.

\section{Standardization.}

Let $\vec{u}=\left\langle u_{0}, \ldots, u_{\nu}\right\rangle$ be a 0 -pedigree for $\langle A, \bar{x}\rangle$. As $\alpha \sqsupset 0$, there is $n$ such that $u_{n} \sqsubset \alpha \sqsubseteq u_{n+1}$ [or $n=\nu$ and $\left.u_{\nu} \sqsubset \alpha\right]$. Let $\beta \boxminus u_{n}$ and let $\mathcal{Q}(W)$ be the $\in$-formula from the Normal Form Theorem such that $\langle y,\langle A, \bar{x}\rangle\rangle \mathbf{M}_{\gamma} W$ implies that $\left(y \in A \wedge \mathcal{P}^{\gamma}(y, A, \bar{x})\right) \Leftrightarrow \mathcal{Q}(W)$.

We note that for all $\beta \sqsubseteq \gamma \sqsubset \alpha$ the $\gamma$-type $V$ of $\langle A, \bar{x}\rangle$ can be taken as $u_{n}$ [the $\gamma$-pedigree is $\left.\left\langle u_{n}, \ldots, u_{\nu}\right\rangle\right]$. Then, for all $y \sqsubseteq \gamma$, the $\gamma$-type of $\langle y,\langle A, \bar{x}\rangle\rangle$ is $V_{y}$, and $\left(y \in A \wedge \mathcal{P}^{\gamma}(y, A, \bar{x})\right) \Leftrightarrow \mathcal{Q}\left(V_{y}\right)$.

We fix $\bar{A} \in \mathbb{S}_{\beta}$ such that $A \subseteq \bar{A}$ and let $B:=\left\{z \in \bar{A}: \mathcal{Q}\left(V_{z}\right)\right\} ; \quad B \in \mathbb{S}_{\beta}$ because $V \in \mathbb{S}_{\beta}$ and $\mathcal{Q}$ is an $\in$-formula.

For $y \sqsubseteq \gamma$ now $y \in B \Leftrightarrow y \in \bar{A} \wedge \mathcal{Q}\left(V_{y}\right) \Leftrightarrow y \in A \wedge \mathcal{P}^{\gamma}(y, A, \bar{x})$.

\section{Corollary 12.16 SST SS $^{\sharp}$ SST $^{*}$.}

Before establishing the converse, we deduce some consequences of GRIST.

First, an immediate consequence of Transfer is that all axioms (and hence, all theorems) of GRIST remain valid if $\sqsubseteq$ is replaced by $\sqsubseteq_{\alpha}$, for any $\alpha$. In other words, $\left(\mathbb{V}, \in, \sqsubseteq_{\alpha}\right) \vDash$ GRIST. In the terminology of [14], GRIST is fully relativized.

Several variations of the theory FRIST are introduced in [13]. Below are the axioms of FRIST as presented in [16]; this version is called FRBST $_{2}$ in [13]. ${ }^{4}$

ZFC (with Separation and Replacement for $\in$-formulas).

Relativization: $\sqsubseteq$ is a dense total pre-ordering with a least element 0 and no greatest element.

\footnotetext{
${ }^{4}$ Actually, FRIST Standardization is stated in [16] with $\left(\forall x \in \mathbb{S}_{0}\right)$ in place of $(\forall x)$. This is an oversight. As in [13], the axiom of Boundedness has to be added to the axioms of [16]. The present version implies Boundedness by the argument in Remark (2) before Proposition 3.1.
} 
Transfer: For all $\alpha,\left(\forall \bar{x} \in \mathbb{S}_{0}\right)\left(\mathcal{P}^{0}(\bar{x}) \Leftrightarrow \mathcal{P}^{\alpha}(\bar{x})\right)$.

Standardization: For all $\bar{x}$,

$$
(\forall x)\left(\exists y \in \mathbb{S}_{0}\right)\left(\forall z \in \mathbb{S}_{0}\right)\left(z \in y \Leftrightarrow z \in x \wedge \mathcal{P}^{0}(z, x, \bar{x})\right) .
$$

Idealization: $\quad$ For all $0 \sqsubset \beta, A, B \in \mathbb{S}_{0}$ and $\bar{x}$,

$$
\left(\forall a \in A^{\text {fin }} \cap \mathbb{S}_{0}\right)(\exists y \in B)(\forall x \in a) \mathcal{P}^{\beta}(x, y, \bar{x}) \Leftrightarrow(\exists y \in B)\left(\forall x \in A \cap \mathbb{S}_{0}\right) \mathcal{P}^{\beta}(x, y, \bar{x}) .
$$

\section{Proposition 12.17 GRIST $\vdash$ FRIST.}

\section{Proof FRIST Standardization:}

Let $\mathcal{R}(x, \bar{x})$ be the formula $(\exists y \sqsubseteq 0)(\forall z \sqsubseteq 0)(z \in y \Leftrightarrow z \in x \wedge \mathcal{P}(z, x, \bar{x}))$. GRIST Standardization implies that $(\forall \alpha \sqsupset 0)(\exists \beta \sqsubset \alpha) \mathcal{R}^{\beta}(x, \bar{x})$ (let $\left.\gamma=\beta\right)$. By Granularity, there is a $\sqsubseteq$-least $\alpha$ such that $\mathcal{R}^{\alpha}(x, \bar{x})$ holds. The two statements together imply that this $\alpha \boxminus 0$, ie, $\mathcal{R}(x, \bar{x})$ holds.

\section{FRIST Idealization:}

Let $\mathcal{Q}(x, y, \bar{x}, \beta)$ be the formula obtained from $\mathcal{P}(x, y, \bar{x})$ by replacing each occurrence of $z \sqsubseteq w$ with $z \sqsubseteq \beta \vee z \sqsubseteq w(z, w$ any variables). Note that, for all $\gamma \sqsubseteq \beta$, $z \sqsubseteq_{\beta} w \Leftrightarrow z \sqsubseteq_{\gamma} \beta \vee z \sqsubseteq_{\gamma} w$, ie, $\mathcal{Q}^{\gamma}(x, y, \bar{x}, \beta) \Leftrightarrow \mathcal{P}^{\beta}(x, y, \bar{x})$.

In this notation, the antecedent of $\Rightarrow$ in FRIST Idealization is $\left(\forall a \in \mathcal{P}^{\mathrm{fin}} A\right)[a \sqsubseteq$ $0 \Rightarrow(\exists y \in B)(\forall x \in a) \mathcal{Q}(x, y, \bar{x}, \beta)]$; let $\mathcal{R}(A, B, \bar{x}, \beta)$ denote this formula. If there is $\gamma \sqsubset \beta$ such that $\neg \mathcal{R}^{\gamma}(A, B, \bar{x}, \beta)$, let $\bar{\beta}$ be the least such $\gamma$ (Granularity); otherwise let $\bar{\beta} \boxminus \beta$. In either case $\bar{\beta} \sqsupset 0$ and $(\forall \gamma \sqsubset \bar{\beta}) \mathcal{R}^{\gamma}(A, B, \bar{x}, \beta)$ holds [this is a special case of Local Transfer; see 12.22]. Hence $(\forall \gamma \sqsubset \bar{\beta})\left(\forall a \in \mathcal{P}^{\text {fin }} A\right)(a \sqsubseteq \gamma \Rightarrow(\exists y \in$ $\left.B)(\forall x \in a) \mathcal{Q}^{\bar{\beta}}(x, y, \bar{x}, \beta)\right)$ [note $\mathcal{Q}^{\gamma} \Leftrightarrow \mathcal{P}^{\beta} \Leftrightarrow \mathcal{Q}^{\bar{\beta}}$ because $\gamma, \bar{\beta} \sqsubseteq \beta$ ]. By GRIST Idealization now there is $y$ such that $(\forall x \in A)\left(x \sqsubset \bar{\beta} \Rightarrow \mathcal{Q}^{\bar{\beta}}(x, y, \bar{x}, \beta)\right)$, and in particular, $(\forall x \in A)\left(x \sqsubseteq 0 \Rightarrow \mathcal{P}^{\beta}(x, y, \bar{x})\right)$.

Corollary 12.18 (GRIST) For every $\alpha,\left(\mathbb{V}, \in, \mathbb{S}_{\alpha}\right) \vDash$ BST.

Proposition 12.19 (GRIST) (External Induction) Let $\mathcal{P}(x, \bar{x})$ be any $\in-\sqsubseteq$-formula. If, for all standard ordinals $\xi, \quad\left(\forall^{\text {st }} \eta<\xi\right) \mathcal{P}(\eta, \bar{x}) \Rightarrow \mathcal{P}(\xi, \bar{x})$ holds, then $\left(\forall^{\text {st }} \xi\right) \mathcal{P}(\xi, \bar{x})$ holds.

In particular, if $\mathcal{P}(0, \bar{x})$ holds and $\mathcal{P}(n, \bar{x}) \Rightarrow \mathcal{P}(n+1, \bar{x})$ holds for all standard $n \in \omega$, then $\left(\forall^{\text {st }} n \in \omega\right) \mathcal{P}(n, \bar{x})$ holds. 
Proof Assume $\neg \mathcal{P}\left(\xi_{0}, \bar{x}\right)$ for some standard $\xi_{0}$. By FRIST Standardization, there is a standard set $X$ such that, for all standard $\eta, \eta \in X \Leftrightarrow \eta \in \xi_{0}+1 \wedge \neg \mathcal{P}(\eta, \bar{x})$. The set $X \neq 0$, because $\xi_{0} \in X$, so it has the least element $\xi_{1}$ and $\xi_{1}$ is standard. This gives a contradiction.

Proposition 12.20 (GRIST) ( $\alpha$-Finite Choice) Let $\mathcal{P}(x, y, \bar{x})$ be an $\in$ - $\sqsubseteq$-formula. If $a \in \mathbb{S}_{\alpha}$ is finite and $(\forall x \in a)(\exists y) \mathcal{P}^{\alpha}(x, y, \bar{x})$, then there exists a function $f$ with $\operatorname{dom} f=a$ such that $(\forall x \in a) \mathcal{P}^{\alpha}(x, f(x), \bar{x})$.

Proof It suffices to prove the assertion for the case when $\alpha \boxminus 0$ [then apply Transfer] and $a=n \in \omega \cap \mathbb{S}_{0}$. This is easily done by External Induction.

\section{Proof that GRIST $\vdash$ SST $^{\sharp}$ :}

GRIST Transfer immediately implies Transfer from/into $\mathbb{S}_{\alpha}$. Inner Standardization into $\mathbb{S}_{\alpha}$ follows from FRIST Standardization, and GRIST Idealization implies Block Idealization (let $\mathcal{P}$ be the formula $y \sqsubseteq 0 \wedge\langle x, y\rangle \in R$ ). The remaining axioms of SST $^{*}$ are included among those of GRIST. SST ${ }^{*}$ implies SST ${ }^{b}$ (Corollary 10.11). It remains to prove $(\forall \alpha)\left(\mathbf{F}_{\alpha}\right)$.

Proof It suffices to prove $\mathbf{F}_{0}$; the general case follows by Transfer. Given $U, V, F \in \mathbb{S}_{0}$ such that $U=\bar{F}(V)$ and $x \mathrm{M}_{0} U$, we need to show that for some $y, x=F(y) \wedge y \mathbf{M}_{0} V$.

We proceed by external induction on rank of $V$. The claim is clear when rank $V=0$. Let $V \in \boldsymbol{\beta}_{\xi} B \backslash \boldsymbol{\beta}_{<\xi} B, U \in \boldsymbol{\beta}_{\xi} A$, and $\left\langle u_{0}, \ldots, u_{\nu}\right\rangle$ be the 0-pedigree for $x$ over $A$ with $u_{0}=U=\bar{F}(V)$. There are two cases to consider.

Case 1. $\bar{F}[V]$ is principal, generated by $U$.

We fix $\beta$ such that $0 \sqsubset \beta \sqsubset u_{1}$ [just $0 \sqsubset \beta$, if $\nu=0$ ]; then also $x \mathbf{M}_{\beta} U$. From $(\forall X)[X \in V \Rightarrow U \in \bar{F}[X] \Rightarrow(\exists v \in X)(\bar{F}(v)=U)]$ it follows, by Block Idealization, that there is $v \in \mathbb{S}_{\beta}$ such that $(\forall X \in V)(X \sqsubset \beta \Rightarrow v \in X \wedge \bar{F}(v)=U)$. By the inductive assumption transferred to level $\beta$ [note that $\operatorname{rank} v<\operatorname{rank} V$ ], there is $y$ such that $x=F(y) \wedge y \mathbf{M}_{\beta} v$. Since $v \mathbb{M}_{\gamma} V$ for all $\gamma \sqsubset \beta$, it follows that $y \mathbf{M}_{0} V$.

Case 2. $\bar{F}[V]$ is nonprincipal.

We let $\beta \boxminus u_{1}[\nu>0]$, so $x \mathbf{M}_{\beta} u_{1}$. Then $\bar{F}[V]=U$ and from $(\forall X \in V)[X \sqsubset \beta \Rightarrow$ $\left.\bar{F}[X] \in U \Rightarrow u_{1} \in \bar{F}[X] \Rightarrow(\exists v \in X)\left(u_{1}=\bar{F}(v)\right)\right]$ it follows, by Block Idealization, that there is some $v \in \mathbb{S}_{\beta}$ such that $(\forall X \in V)\left[X \sqsubset \beta \Rightarrow v \in X \wedge \bar{F}(v)=u_{1}\right]$. By the inductive assumption transferred to level $\beta$ again, there is $y$ such that $x=F(y) \wedge y \mathbf{M}_{\beta} v$ and hence $y \mathbf{M}_{0} V$. 


\subsection{Consequences of GRIST.}

In this subsection we derive a number of further consequences of GRIST. Some of them throw additional light on the structure of the theory. Others have been found useful for development of analysis in the framework of GRIST [17]. Some of these consequences are generalizations of principles derived from RIST by Péraire and from BST by Kanovei and Reeken; in those cases, the original formulations can be found in the indicated references. The last few propositions give examples of principles valid in BST but whose analogs for $\epsilon-\sqsubseteq$-formulas fail in GRIST [of course, the original versions for $\in$-st-formulas hold, as all $\left(\mathbb{V}, \in, \mathbb{S}_{\alpha}\right)$ satisfy BST].

Proposition 12.21 (Support Principle) Given a formula $\mathcal{P}\left(x_{1}, \ldots, x_{k}\right)$ in the $\in-\sqsubseteq$ language and sets $x_{1}, \ldots, x_{k}$, there is a level set $\left\{\alpha_{0}, \alpha_{1}, \ldots, \alpha_{n}\right\}$ such that $\alpha_{0} \boxminus 0 \sqsubset$ $\alpha_{1} \sqsubset \ldots \sqsubset \alpha_{n}$ and for all $i \leq n$ and all $\beta$ with $\alpha_{i} \sqsubseteq \beta \sqsubset \alpha_{i+1}$

$$
\mathcal{P}^{\alpha_{i}}(\bar{x}) \Leftrightarrow \mathcal{P}^{\beta}(\bar{x}) \Leftrightarrow \neg \mathcal{P}^{\alpha_{i+1}}(\bar{x}) .
$$

Proof Let $\mathcal{Q}$ be the formula corresponding to $\mathcal{P}$ by the Normal Form Theorem and let $\vec{u}=\left\langle u_{0}, \ldots, u_{\nu}\right\rangle$ be the 0 -pedigree for $\langle\bar{x}\rangle$. Recall that $\langle\bar{x}\rangle \mathbf{M}_{\beta} u_{n}$ for all $u_{n} \sqsubseteq \beta \sqsubset u_{n+1}$ and hence $\mathcal{P}^{u_{n}}(\bar{x}) \Leftrightarrow \mathcal{Q}\left(u_{n}\right) \Leftrightarrow \mathcal{P}^{\beta}(\bar{x})$. Define recursively: $\alpha_{0}:=u_{0} \boxminus 0$; if $\alpha_{i-1} \boxminus u_{m}$, let $\alpha_{i}=u_{n}$ for the least $n>m$ (if any) such that $\mathcal{Q}\left(u_{m}\right) \Leftrightarrow \neg \mathcal{Q}\left(u_{n}\right)$.

Proposition 12.22 (Local Transfer) Let $\mathcal{P}\left(x_{1}, \ldots, x_{k}, x_{k+1}, \ldots, x_{n}\right)$ be any $\in-\sqsubseteq$ formula. For any sets $x_{k+1}, \ldots, x_{n}$ and any $\alpha$ there is $\gamma \sqsupset \alpha$ such that, for all $\alpha \sqsubseteq \beta \sqsubset \gamma$ and all $x_{1}, \ldots, x_{k} \sqsubseteq \alpha, \quad \mathcal{P}^{\alpha}\left(x_{1}, \ldots, x_{n}\right) \Leftrightarrow \mathcal{P}^{\beta}\left(x_{1}, \ldots, x_{n}\right)$.

Proof Without loss of generality we can assume $k=1, n=2$, and write $a$ for $x_{1}, x$ for $x_{2}$. If $x \sqsubseteq \alpha, \mathcal{P}^{\alpha}(a, x) \Leftrightarrow \mathcal{P}^{\beta}(a, x)$ holds for all $\beta \sqsupseteq \alpha$ and all $a \sqsubseteq \alpha$ by Transfer. Otherwise, let $\mathcal{Q}(a, U)$ be the $\in$-formula corresponding to $\mathcal{P}(a, x)$ by the Boldface Normal Form Theorem. Let $\vec{u}=\left\langle u_{0}, \ldots, u_{\nu}\right\rangle$ be an $\alpha$-pedigree for $x$ and let $U:=u_{0}$, so that $x \mathbf{M}_{\alpha} U$. Note that $\nu>0$ and let $\gamma \boxminus u_{1} \sqsupset \alpha$. If $\alpha \sqsubseteq \beta \sqsubset \gamma$, then $\vec{u}$ is also a $\beta$-pedigree for $x$, so $x \mathbf{M}_{\beta} U$ holds, and $\mathcal{P}^{\alpha}(a, x) \Leftrightarrow \mathcal{Q}(a, U) \Leftrightarrow \mathcal{P}^{\beta}(a, x)$, for all $a \sqsubseteq \alpha$.

Here is another version of Standardization.

Proposition 12.23 For any $\alpha \sqsupset 0$ and any $A, x_{1}, \ldots, x_{k}$, there exists $B \sqsubset \alpha$ such that $(\forall y \sqsubset \alpha)\left(y \in B \Leftrightarrow y \in A \wedge \mathcal{P}^{\alpha}\left(y, A, x_{1}, \ldots, x_{k}\right)\right)$. 
Proof As in the proof of FRIST Idealization in 12.17 [with $\alpha$ in place of $\beta$ ], let $\mathcal{Q}$ be the formula obtained from $\mathcal{P}$ by replacing each occurrence of $z \sqsubseteq w$ with $z \sqsubseteq \alpha \vee z \sqsubseteq w$ Applying Standardization to $\mathcal{Q}$, we get $B \sqsubseteq \beta \sqsubset \alpha$ such that, for all $\beta \sqsubseteq \gamma \sqsubset \alpha, \quad(\forall y \sqsubseteq \gamma)\left(y \in B \Leftrightarrow y \in A \wedge \mathcal{Q}^{\gamma}(y, A, \bar{x})\right)$, and consequently $(\forall y \sqsubset \alpha)\left(y \in B \Leftrightarrow y \in A \wedge \mathcal{P}^{\alpha}(y, A, \bar{x})\right)$.

Proposition 12.24 Let $V \in \boldsymbol{\beta}_{n} B \backslash \boldsymbol{\beta}_{n-1} B$ for $n \in \mathbb{S}_{0}, n>0, \quad V \in \mathbb{S}_{0}$. For every level set $\left\{\alpha_{0}, \alpha_{1}, \ldots, \alpha_{n}\right\}$ such that $\alpha_{0} \boxminus 0 \sqsubset \alpha_{1} \sqsubset \ldots \sqsubset \alpha_{n}$ there is $z \mathbf{M}_{0} V$ such that every pedigree $\left\langle u_{0}, \ldots, u_{\nu}\right\rangle$ for $z$ has $\nu=n$ and $u_{i} \boxminus \alpha_{i}$, for all $i \leq n$.

Proof By External Induction.

The statement is true for $n=1$ by Proposition 12.15 .

Assume it is true for $n$ and let $V \in \boldsymbol{\beta}_{n+1} B \backslash \boldsymbol{\beta}_{n} B$. Then there is $v_{1} \mathbb{M}_{0} V$ such that $v_{1} \boxminus \alpha_{1}$ and $\left\langle V, v_{1}\right\rangle$ is a pedigree for $v_{1}$ over $\boldsymbol{\beta}_{n} B$, by Proposition 12.15 where we regard $V$ as an element of $\boldsymbol{\beta}\left(\boldsymbol{\beta}_{n} B\right) \backslash \boldsymbol{\beta}_{n} B$. We have $v_{1} \in \boldsymbol{\beta}_{n} B \backslash \boldsymbol{\beta}_{n-1} B$, as $\boldsymbol{\beta}_{n} B \backslash \boldsymbol{\beta}_{n-1} B \in V$. The statement for $n$ transfers to level $\alpha_{1} \boxminus v_{1}$, where $\left\{\alpha_{1} \sqsubset \ldots \sqsubset \alpha_{n}\right\}$ is an $\alpha_{1}$-level set of cardinality $n$. Hence, by inductive assumption, there is $z \mathbf{M}_{\alpha_{1}} v_{1}$ such that an $\alpha_{1}$-pedigree $\vec{u}=\left\langle u_{1}, \ldots u_{n}\right\rangle$ for $z$ over $B$ has $u_{1}=v_{1}$ and $u_{2} \boxminus \alpha_{2}, \ldots, u_{n} \boxminus \alpha_{n}$. Clearly $\left\langle u_{0}:=V, u_{1}, \ldots, u_{n}\right\rangle$ is a pedigree for $z$ over $B$ and $u_{i} \boxminus \alpha_{i}$, for all $i \leq n$.

Definition 12.25 $\mathcal{P}\left(x_{1}, \ldots, x_{k} ; y_{1}, \ldots, y_{n}\right)$ denotes a formula of the $\in$ - $\sqsubseteq$-language where the variables $y_{1}, \ldots, y_{n}$ appear only in the scope of $\sqsubseteq$ [not $\in$ or $\left.=\right]$.

Proposition 12.26 (Polytransfer; Péraire [29])

Let $\alpha \sqsubset \alpha_{1} \sqsubset \ldots \sqsubset \alpha_{n}$ and $\beta \sqsubset \beta_{1} \sqsubset \ldots \sqsubset \beta_{n}$. Then

$\left(\forall x_{1}, \ldots, x_{k} \in \mathbb{S}_{\alpha} \cap \mathbb{S}_{\beta}\right)\left(\mathcal{P}^{\alpha}\left(x_{1}, \ldots, x_{k} ; \alpha_{1}, \ldots, \alpha_{n}\right) \Leftrightarrow \mathcal{P}^{\beta}\left(x_{1}, \ldots, x_{k} ; \beta_{1}, \ldots, \beta_{n}\right)\right)$.

Proof Without loss of generality we assume $k=1$ and write $a$ for $x_{1}$. Fix $V \in$ $\mathbb{S}_{0} \cap\left(\boldsymbol{\beta}_{n} B \backslash \boldsymbol{\beta}_{n-1} B\right)$. By Proposition 12.24 there are $z \mathbf{M}_{0} V, z^{\prime} \mathbf{M}_{0} V$ and their pedigrees $\vec{u}=\left\langle u_{0}, \ldots, u_{n}\right\rangle$ and $\vec{u}^{\prime}=\left\langle u_{0}^{\prime}, \ldots, u_{n}^{\prime}\right\rangle$, resp., such that $u_{1} \boxminus \alpha_{1}, \ldots, u_{n} \boxminus \alpha_{n}$, $u_{1}^{\prime} \boxminus \beta_{1}, \ldots, u_{n}^{\prime} \boxminus \beta_{n}, u_{0}=V=u_{0}^{\prime}$. Note that $\vec{u}$ is an $\alpha$-pedigree for $z$ and $\vec{u}^{\prime}$ is a $\beta$-pedigree for $z$ as well. Then clearly

$$
\begin{gathered}
\mathcal{P}^{\alpha}\left(a ; \alpha_{1}, \ldots, \alpha_{n}\right) \Leftrightarrow \mathcal{P}^{\alpha}\left(a ; u_{1}, \ldots, u_{n}\right) \Leftrightarrow \mathcal{P}^{\alpha}(a, z) \quad \text { and } \\
\mathcal{P}^{\beta}\left(a ; \beta_{1}, \ldots, \beta_{n}\right) \Leftrightarrow \mathcal{P}^{\beta}\left(a ; u_{1}^{\prime}, \ldots, u_{n}^{\prime}\right) \Leftrightarrow \mathcal{P}^{\beta}\left(a, z^{\prime}\right)
\end{gathered}
$$

where $\mathcal{P}(x, z)$ is the formula expressing $(\exists \vec{u})\left[\vec{u}\right.$ is a pedigree for $z \wedge u=\left\langle u_{0}, \ldots, u_{n}\right\rangle$ $\left.\wedge \mathcal{P}\left(x ; u_{1}, \ldots, u_{n}\right)\right]$. Let $\mathcal{Q}(a, U)$ be the formula corresponding to $\mathcal{P}(x, z)$ by the Boldface Normal Form Theorem. As $z \mathbf{M}_{\alpha} V, z \mathbf{M}_{\beta} V$, and $a \in \mathbb{S}_{\alpha} \cap \mathbb{S}_{\beta}, \mathcal{P}^{\alpha}(a, z) \Leftrightarrow$ $\mathcal{Q}(a, V) \Leftrightarrow \mathcal{P}^{\beta}\left(a, z^{\prime}\right)$. 
Proposition 12.27 (Partial Transfer; Péraire [29]) For any $\alpha \sqsubset \beta$, $\left(\forall \bar{x} \in \mathbb{S}_{\alpha}\right)\left[(\exists x)\left(\mathcal{P}^{\alpha}(x, \bar{x} ; \alpha) \wedge \mathcal{Q}^{\alpha}(x, \bar{x} ; \alpha)\right) \Rightarrow(\exists x)\left(\mathcal{P}^{\alpha}(x, \bar{x} ; \alpha) \wedge \mathcal{Q}^{\beta}(x, \bar{x} ; \beta)\right)\right]$.

Proof Fix $\alpha, \bar{x} \in \mathbb{S}_{\alpha}$, and $x$ such that $\mathcal{P}^{\alpha}(x, \bar{x} ; \alpha) \wedge \mathcal{Q}^{\alpha}(x, \bar{x} ; \alpha)$. By Local Transfer there is some $\beta^{\prime} \sqsupset \alpha$ such that $\mathcal{Q}^{\beta^{\prime}}\left(x, \bar{x} ; \beta^{\prime}\right)$ holds, ie, $(\exists x)\left(\mathcal{P}^{\alpha}(x, \bar{x} ; \alpha) \wedge \mathcal{Q}^{\beta^{\prime}}\left(x, \bar{x} ; \beta^{\prime}\right)\right)$ holds. Given $\beta \sqsupset \alpha$, apply Polytransfer to the sequences of levels $\alpha \sqsubset \beta^{\prime}$ and $\alpha \sqsubset \beta$ to obtain $(\exists x)\left(\mathcal{P}^{\alpha}(x, \bar{x} ; \alpha) \wedge \mathcal{Q}^{\beta}(x, \bar{x} ; \beta)\right)$.

Proposition 12.28 ( $\alpha$-Standard Size Choice; Kanovei and Reeken [22])

Let $\mathcal{P}\left(x, y, x_{1}, \ldots, x_{k}\right)$ be an $\in-\sqsubseteq$-formula. For every $\alpha$ and every $A \in \mathbb{S}_{\alpha}$ such that $\left(\forall x \in A \cap \mathbb{S}_{\alpha}\right)(\exists y) \mathcal{P}^{\alpha}\left(x, y, x_{1}, \ldots, x_{k}\right)$ there exists a function $f$ with $\operatorname{dom} f=A$ such that $\left(\forall x \in A \cap \mathbb{S}_{\alpha}\right) \mathcal{P}^{\alpha}\left(x, f(x), x_{1}, \ldots, x_{k}\right)$.

Proof We fix $\bar{x}=x_{1}, \ldots, x_{k}, \alpha$, and $A$ satisfying the assumptions. Let $\mathcal{P}^{\prime}(z, y)$ be the formula $(\exists x, \bar{x})[z=\langle x,\langle\bar{x}\rangle\rangle \wedge \mathcal{P}(x, y, \bar{x})]$, and let $\mathcal{Q}(V)$ be the $\in$-formula corresponding to $\mathcal{P}^{\prime}$ by the Normal Form Theorem. If $\langle\bar{x}\rangle \mathbf{M}_{\alpha} U$ and $x \in \mathbb{S}_{\alpha}$, we then have $(\exists y) \mathcal{P}^{\alpha}(x, y, \bar{x}) \Leftrightarrow(\exists V)\left[\bar{\pi}_{1}(V)=U_{x} \wedge Q(V)\right]$ [see Proposition 9.7 and the proof of $\exists$ step of the Normal Form Theorem]. By ZFC Selection, which holds in $\left(\mathbb{S}_{\alpha}, \in\right)$, there are functions $V, B \in \mathbb{S}_{\alpha}$, defined on $A$, such that $V(x) \in \boldsymbol{\beta}_{\infty} B(x)$ and $(\forall x \in A)\left[\pi_{1}(V(x))=U_{x} \wedge \mathcal{Q}(V(x))\right]$. It remains to prove the following.

Claim. There is a function $\vec{v}$ with $\operatorname{dom} \vec{v}=A$ such that $\left(\forall x \in A \cap \mathbb{S}_{\alpha}\right)[\vec{v}(x)=$ $\left\langle v(x)_{0}, \ldots, v(x)_{\nu(x)}\right\rangle$ is an $\alpha$-pedigree over $B(x)$ with $v(x)_{0}=V(x)$ and $v(x)_{\nu(x)}=$ $\langle\langle x,\langle\bar{x}\rangle\rangle, y(x)\rangle$ for some (uniquely determined) $y(x)$.

The function $f$ on $A$ defined by $x \mapsto y(x)$ then has the property that, for all $x \in A \cap \mathbb{S}_{\alpha}$, $\langle\langle x,\langle\bar{x}\rangle\rangle, f(x)\rangle \mathbf{M}_{\alpha} V(x) \wedge \mathcal{Q}(V(x))$, so $\left(\mathcal{P}^{\prime}\right)^{\alpha}(\langle x,\langle\bar{x}\rangle\rangle, f(x))$ holds, ie, $\mathcal{P}^{\alpha}(x, f(x), \bar{x})$ holds.

Proof of Claim.

For every $x \in A \cap \mathbb{S}_{\alpha}$ there is some $\alpha$-pedigree $\vec{v}$ over $B(x)$ with $v_{0}=V(x)$ and $v_{\nu(x)}=\langle\langle x,\langle\bar{x}\rangle\rangle, y\rangle$ for some $y$. By Local Transfer 12.22, there is $\beta \sqsupset \alpha$ such that for every $x \in A \cap \mathbb{S}_{\alpha}$ there is some $\beta$-pedigree $\vec{v}$ over $B(x)$ with $v_{0}=V(x)$ and $v_{\nu(x)}=\langle\langle x,\langle\bar{x}\rangle\rangle, y\rangle$ for some $y$. By $\alpha$-Finite Choice, for every $a \in \mathcal{P}^{\mathrm{fin}} A \cap \mathbb{S}_{\alpha}$ there is a function $\vec{v}$ such that, for every $x \in a, \vec{v}(x)$ is a $\beta$-pedigree over $B(x)$ with $v(x)_{0}=V(x)$ and $v(x)_{\nu(x)}=\langle\langle x,\langle\bar{x}\rangle\rangle, y(x)\rangle$. Using Idealization we obtain a function $\vec{v}$ with $\operatorname{dom} \vec{v}=A$ such that, for every $x \in a, \vec{v}(x)$ is a $\beta$-pedigree over $B(x)$ with $v(x)_{0}=V(x)$ and $v(x)_{\nu(x)}=\langle\langle x,\langle\bar{x}\rangle\rangle, y(x)\rangle$. Finally, as $v(x)_{0}=V(x) \sqsubseteq \alpha$ and $v(x)_{1} \sqsupset \beta \sqsupset \alpha$ [if $\nu(x)>0$ ], $\vec{v}(x)$ is an $\alpha$-pedigree. 
Proposition 12.29 (Map Standardization; Kanovei and Reeken [22]) For every $A \in \mathbb{S}_{\alpha}$ there exists $f \in \mathbb{S}_{\alpha}$ such that $\operatorname{dom} f=A$ and

$$
\left(\forall x \in A \cap \mathbb{S}_{\alpha}\right)\left[\left(\exists y \in \mathbb{S}_{\alpha}\right) \mathcal{P}^{\alpha}(x, y, \bar{x}) \Rightarrow \mathcal{P}^{\alpha}(x, f(x), \bar{x})\right] .
$$

Proof It suffices to give a proof for $\alpha \boxminus 0$ and then use Transfer. Let $\mathcal{R}(x, z, \bar{x})$ be the formula " $z \in \mathbb{O} n \wedge\left[\left(\exists y \in \mathbb{S}_{0}\right)\left(y \in \mathbb{V}_{z} \wedge \mathcal{P}(x, y, \bar{x})\right) \vee\left(\forall y \in \mathbb{S}_{0}\right) \neg \mathcal{P}(x, y, \bar{x})\right]$ " [ $\mathbb{V}_{z}$ is the $z$-th level of the cumulative hierarchy]. By 0-Standard Size Choice, there exists a function $g$ with $\operatorname{dom} g=A$ such that $\left(\forall^{\text {st }} x \in A\right) \mathcal{R}(x, g(x))$. Let $\xi \in \mathbb{O} n \cap \mathbb{S}_{0}$ be such that $\operatorname{ran} g \subseteq \xi$. Then $\left(\forall^{\text {st }} x \in A\right)\left[\left(\exists^{\text {st }} y\right) \mathcal{P}(x, y, \bar{x}) \Rightarrow\left(\exists^{\text {st }} y \in \mathbb{V}_{\xi}\right) \mathcal{P}(x, y, \bar{x})\right]$. By FRIST Standardization we obtain $C \in \mathbb{S}_{0}$ such that, for all $x, y \in \mathbb{S}_{0},\langle x, y\rangle \in C \Leftrightarrow$ $x \in A \wedge y \in \mathbb{V}_{\xi} \wedge \mathcal{P}(x, y, \bar{x})$. Using Axiom of Choice in $(\mathbb{S}, \in)$, we obtain a function $f \in \mathbb{S}_{0}$ with $\operatorname{dom} f=A$ such that $(\forall x \in A)[(\exists y)(\langle x, y\rangle \in C) \Rightarrow\langle x, f(x)\rangle \in C]$. For this function, $\left(\forall^{\text {st }} x \in A\right)\left[\left(\exists^{\text {st }} y\right) \mathcal{P}(x, y, \bar{x}) \Rightarrow \mathcal{P}(x, f(x), \bar{x})\right]$ then holds.

Proposition 12.30 (Unique Definability; Kanovei and Reeken [22])

If $x$ is uniquely definable in GRIST from parameters in $\mathbb{S}_{0}$, then $x \in \mathbb{S}_{0}$. If $x \notin \mathbb{S}_{0}$, then for each $\alpha \sqsupset 0$ there exist $y \boxminus \alpha$ such that $x$ and $y$ are $\in-\sqsubseteq$-indiscernible.

Proof Let $x \mathbf{M}_{0} U$. If $\operatorname{rank} U=0$, then $U=x$ and $x \in \mathbb{S}_{0}$.

If rank $U>0$, we prove that for every $\gamma \sqsupset 0$ there is $y \boxminus \gamma$ such that $y \mathbf{M}_{0} U$, and hence all such $y$ are $\in$ - $\sqsubseteq$-indiscernible.

We fix $V \in \boldsymbol{\beta}_{1} B \backslash \boldsymbol{\beta}_{0} B, V \in \mathbb{S}_{0}$, and use Proposition 12.15 to obtain $z \boxminus \gamma$ such that ${ }_{z} \mathbf{M}_{0} V$. As $\overline{\pi_{2}}\left(U^{\sim} V\right)=V$, by $\mathbf{F}_{0}$ there is $y$ such that $\langle y, z\rangle \mathbf{M}_{0} U^{\sim} V$. As $\overline{\pi_{1}}\left(U^{\sim} V\right)=U$, $y \mathbf{M}_{0} U$ holds. Let $y \boxminus \beta ; \beta \sqsupset 0$. In the course of proof of Proposition 12.15 [let $\alpha=0$ there] we established:

If $x \boxminus \beta, \beta \sqsupset 0, x \mathbf{M}_{0} U$ and $\langle x, z\rangle \mathbf{M}_{0} U^{\sim} V$, then $z \boxminus x$.

Applying this to $y$ in place of $x$, we get $y \boxminus z \boxminus \gamma$.

Proposition 12.31 If $A \neq 0$, then there is $x \in A$ such that $x \boxminus A$.

Proof If $A \sqsubseteq 0$, the assertion is true by Transfer into $\mathbb{S}_{0}$, so we assume $A \sqsupset 0$. Let $\beta \boxminus A$ and fix $\widetilde{A} \in \mathbb{S}_{0}$ such that $A \subseteq \widetilde{A}$. If there is $a \in \mathcal{P}^{\text {fin }} \widetilde{A}$ such that $a \sqsubset \beta$ and $A \subseteq a$, then $A \sqsubseteq a \sqsubset \beta$, a contradiction. Therefore $(\forall a \in \mathcal{P}$ fin $\widetilde{A})[a \sqsubset \beta \Rightarrow(\exists y)(\forall x \in$ $a)\left(y \neq x \wedge y \in A \wedge y \sqsubseteq_{\beta} 0\right)$ ]. [The $y \sqsubseteq \beta$ clause follows by Transfer into $\mathbb{S}_{\beta}$.] By Idealization there is $y$ such that $x \sqsubset \beta \Rightarrow y \neq x \wedge y \in A \wedge y \sqsubseteq \beta 0$ for all $x \in A$, ie, $y \in A$ and $y \boxminus \beta$. 
Proposition 12.32 (Levels of Elements of Sets )

(a) If $A$ is infinite, then $(\forall \beta \sqsupseteq A)(\exists x)(x \in A \wedge x \boxminus \beta)$.

(b) $(\forall x \in A)(x \sqsubseteq \alpha)$ if and only if $A$ is finite and $A \sqsubseteq \alpha$.

Proof (a) If $A$ is infinite and $A \boxminus \alpha$, then there is a one-one mapping $f \in \mathbb{S}_{\alpha}$ of $\omega$ onto $A$. By Proposition 12.15, for every $\beta$ there is $\nu \in \omega$ such that $\nu \boxminus \beta$. For $\beta \sqsupset \alpha$ then $\beta \boxminus \nu \boxminus f(\nu) \in A$. For $\beta \boxminus \alpha$ the existence of $a \in A, a \boxminus \beta$, follows from the preceding proposition.

(b) is a consequence of (a), the preceding proposition, and Proposition 3.11.

Proposition 12.33 Collection Principle for $\epsilon-\sqsubseteq$-formulas fails in GRIST.

Proof Let $\mathcal{P}(n, U)$ be the formula $n \mathbf{M}_{0} U$. By $\mathbf{B}_{0},(\forall n \in \omega)\left(\exists ! U \in \boldsymbol{\beta}_{\infty} \omega\right) \mathcal{P}(n, U)$. By $\mathbf{F}_{0},\left(\forall U \in \boldsymbol{\beta}_{\infty} \omega\right)(\exists n \in \omega) \mathcal{P}(n, U)$. But $\boldsymbol{\beta}_{\infty} \omega$ is a proper class.

This corollary illustrates the one principal metamathematical difference between BST and GRIST. In BST, the collection of all types of elements of a set $A$ is a set $\boldsymbol{\beta} A$. Because of this, BST can be extended to a theory of external sets, HST, that satisfies Collection and other useful axioms. In GRIST it is a proper class $\boldsymbol{\beta}_{\infty} A$. The failure of Collection implies the impossibility of extending GRIST to an HST-like theory. The issue of external sets is discussed further in the Conclusion.

Kanovei and Reeken [22] formulated a second version of Idealization, which they call Local Idealization, and showed that it holds in BST. An analogous principle for the $\in$ - $\sqsubseteq$-language is false in GRIST.

Local Idealization: For all $\alpha \sqsubset \beta, B \sqsubseteq \alpha$, and all $x_{1}, \ldots, x_{k}$,

$$
\begin{gathered}
(\forall \text { finite } a)\left[a \sqsubseteq \alpha \Rightarrow(\exists y \in B)(\forall x \in a) \mathcal{P}^{\beta}\left(x, y, B, x_{1}, \ldots, x_{k}\right)\right] \\
\Leftrightarrow(\exists y \in B)(\forall x)\left[x \sqsubseteq \alpha \Rightarrow \mathcal{P}^{\beta}\left(x, y, B, x_{1}, \ldots, x_{k}\right)\right] .
\end{gathered}
$$

Proposition 12.34 Local Idealization fails in GRIST.

Proof Fix $\beta \sqsupset 0$. For every finite $\left\{U_{1}, \ldots, U_{n}\right\} \subseteq \boldsymbol{\beta}_{\infty} \omega$ in $\mathbb{S}_{0}$ there is $x \in \omega$ such that each $U_{i}=\bar{f}(V)$ for some $f \in \mathbb{S}_{\beta}$, where $V:=\operatorname{tp}_{\beta}(x ; \omega)$. [Indeed: fix $x_{i}$ such that $U_{i}=\operatorname{tp}_{\beta}\left(x_{i} ; \omega\right)$, let $y:=\left\langle x_{1}, \ldots, x_{n}\right\rangle$, and $U:=\operatorname{tp}_{\beta}\left(y ; \omega^{n}\right)$; then $U_{i}=\overline{\pi_{i}}(U)$. Then let $V:=\bar{\varphi}(U)$ and $x=\varphi(y)$, for some one-one mapping $\varphi \in \mathbb{S}_{0}$ of $\omega^{n}$ onto $\omega$.] Local Idealization implies existence of $x \in \omega$ such that every $U \in \boldsymbol{\beta}_{\infty} \omega \cap \mathbb{S}_{0}$ is of the form $U=\bar{f}(V)$ for some $f \in \mathbb{S}_{\beta}$, where $V:=\operatorname{tp}_{\beta}(x ; \omega)$. However, this means that every $U \in \boldsymbol{\beta}_{\infty} \omega \cap \mathbb{S}_{0}$ has rank less than or equal to rank $V$, a contradiction. 
Remarks It is shown in [13], Theorem 5.1, that FRIST where Idealization is replaced by Local Idealization (the theory called FRBST $_{1}$ in [13]) is a conservative extension of $\mathbf{Z F C}$; in the interpretation given there Granularity holds as well.

Proposition 12.35 Dependent Choice fails in GRIST.

Proof Let $\mathcal{R}(x, y)$ be the formula $x \sqsubset y$. Dependent Choice would imply that:

(*) There is a sequence $\vec{a}=\left\langle a_{n}: n \in \omega\right\rangle$ such that $a_{n} \sqsubset a_{n+1}$

holds for all $n \in \omega$. We show that this is not possible.

Let $\vec{a}$ be such a sequence; then (*) holds in $\left(\mathbb{S}_{0}[[\vec{a}]], \in, \sqsubseteq\right)$, which is isomorphic to $\mathcal{U} \ell t(\mathbb{V} ; \mathbf{U})^{\mathbb{S}_{0}}$ for $\vec{a} \mathbf{M}_{0} U$. We get a contradiction by proving [in $\mathbf{Z F C}$ ] that (*) fails in every $\mathcal{U} \ell t(\mathbb{V} ; \mathcal{U})$

Let $\mathcal{U}$ be of the smallest rank for which $\left(^{*}\right)$ holds; clearly $\operatorname{rank} \mathcal{U}>0$. Note that $0 \sqsubset a_{n}$ for $n>0\left[a_{n-1} \sqsubset a_{n} \boxminus 0\right.$ is impossible]; hence, with $D_{1}:=D_{T^{(1)}}$, we have $\left(\mathcal{U} \ell(\mathbb{V} ; \mathcal{U}), \sqsubseteq_{D_{1}}\right) \vDash(\forall n \geq 1)\left(a_{n} \sqsubset a_{n+1}\right)$, ie, $\left(\mathcal{U} \ell(\mathbb{V} ; \mathcal{U}), \sqsubseteq_{D_{1}}\right) \vDash(*)$. By the Factoring Theorem, $\mathcal{U} \ell\left(\left\langle\mathcal{U} \ell t\left(\mathbb{V} ; \mathcal{U}_{\langle i\rangle}\right): i \in I\right\rangle ; \mathcal{U}(0)\right) \vDash(*)$, ie, $\left\{i \in I: \mathcal{U} \ell t\left(\mathbb{V} ; \mathcal{U}_{\langle i\rangle}\right) \vDash\left(^{*}\right)\right\} \in$ $\mathcal{U}(0)$. This is a contradiction with the inductive assumption.

\subsection{Variations of GRIST.}

\section{(1) Unbounded GRIST.}

Unlike BST or GRIST, Nelson's IST allows Idealization without any bounds on the variables $x$ and $y$; this feature contradicts Boundedness. An extensive discussion of the comparative advantages of IST and BST can be found in [22]; in particular, Kanovei and Reeken showed that IST does not have a realization in ZFC.

It is possible to formulate a GRIST-like theory that extends IST; we call it Unbounded GRIST. Its axioms are obtained from those of GRIST by

(1) replacing Idealization with Unbounded Idealization:

For all $A \sqsubset \beta$ and all $\bar{x}$,

$(\forall$ finite $a \sqsubset \beta)(\exists y)(\forall x \in a) \mathcal{P}^{\beta}(x, y, \bar{x}) \Leftrightarrow(\exists y)(\forall x \sqsubset \beta) \mathcal{P}^{\beta}(x, y, \bar{x})$, and

(2) weakening Standardization [to allow for unbounded sets, without shadows] by replacing "any $A$ " with "any $A \sqsubseteq \alpha$ ".

Unbounded GRIST is an extension of the theory called FRIST in [13]. 
Theorem 12.36 Unbounded GRIST is a conservative extension of ZFC.

Proof As in [13], Proof of Theorem 4.7, we work in the theory $\mathbf{T}$ whose primitives are $\in$ and a constant symbol $v$, and whose axioms are:

ZFC (Separation and Replacement for formulas in the language of $\mathbf{T}$ )

$(\exists \theta)\left(v=\mathbb{V}_{\theta}\right)$

$\left(\forall x_{1}, \ldots, x_{n} \in v\right)\left(\mathcal{P}\left(x_{1}, \ldots, x_{n}\right) \Leftrightarrow \mathcal{P}^{v}\left(x_{1}, \ldots, x_{n}\right)\right)$

where $\mathcal{P}$ is any $\in$-formula, and $\mathcal{P}^{v}$ is the formula obtained from $\mathcal{P}$ by restricting all quantifiers to $v$.

It is well-known (and an immediate consequence of Reflection Principle in ZFC) that $\mathbf{T}$ is a conservative extension of $\mathbf{Z F C}$. The interpretation $\Im_{\Lambda}(\mathbb{V})=\left(\mathbb{V}^{*},=^{*}, \in^{*}, \sqsubseteq^{*}\right)$ for GRIST can thus be constructed in T. We let $\mathbf{v}:=\left\{f \in \mathbb{V}^{*}: f \in^{*} \mathfrak{k}(v)\right\}$. It is straightforward to verify that the restriction of $\Im_{\Lambda}(\mathbb{V})$ to $\mathbf{v}$ is an interpretation of Unbounded GRIST in T.

Remarks The Back and Forth Property is of course incompatible with Unbounded GRIST. Specifically, $\mathbf{B}_{0}$ fails in this interpretation.

\section{(2) Discrete GRIST.}

This is the theory obtained from GRIST by replacing

Density: $(\forall x, y)(x \sqsubset y \Rightarrow(\exists z)(x \sqsubset z \sqsubset y))$ with

\section{Discreteness:}

$(\forall x)(\exists y)(x \sqsubset y \wedge \neg(\exists z)(x \sqsubset z \sqsubset y)) \wedge(\forall x \sqsupset 0)(\exists y)(y \sqsubset x \wedge \neg(\exists z)(y \sqsubset z \sqsubset x))$.

Theorem 12.37 Discrete GRIST has a realization in ZFC.

We do not give a proof of this theorem. The key idea is to reformulate the Back and Forth Property in a form valid for discrete preorderings of levels. This requires, first of all, a re-definition of types. An $\alpha$-type over $A$ is now a pair $(U, L)$ where $U$ is a stratified ultrafilter over $A$ and $L$ is a function defined on $\Sigma T_{\mathbf{U}}$ (see Definition 9.8) such that $L\left(\left\langle U_{1}, \ldots, U_{n}\right\rangle\right)=\left\langle\ell_{1}, \ldots, \ell_{n}\right\rangle$ where each $\ell_{i} \in \omega \cup\{\infty\}$. The idea is that $\ell_{i}$ fixes the number of levels between $U_{i-1}$ and $U_{i}$ (with $U_{0}:=U$ ).

One can suitably modify the developments of Sections 9 - 12 for this notion of type. In particular, an interpretation for Discrete GRIST is given by $\Im_{\Lambda}(\mathbb{V})$ where $\Lambda$ is the usual ordering $(\omega,<)$ of natural numbers. 


\section{Conclusion: GRIST and External Sets.}

Nonstandard analysis traditionally distinguishes between internal and external sets. GRIST is a theory of internal sets only, just like BST and IST. Nelson and his followers demonstrated that extensive work in nonstandard mathematics can be carried out within IST. Many such "internal" arguments become easier and/or more natural in GRIST. Yet, most practitioners consider constructions that fundamentally depend on external sets, such as nonstandard hulls and Loeb measures, to be an essential part of nonstandard analysis. This is one reason why we need to address the issue of extendibility of GRIST to a theory of external sets.

There are also foundational reasons. Theories like BST and IST exhibit a curious lack of symmetry: among their three major principles, Standardization alone admits arbitrary formulas (of the $\in$-st-language); Transfer and Idealization require $\in$-formulas. The desire to make all of these principles applicable to arbitrary formulas was one of the original motivations for FRIST. An immediate consequence of Transfer for all formulas is "Full Relativization": A valid statement of FRIST (or GRIST) remains valid upon replacing $\sqsubseteq$ by $\sqsubseteq_{\alpha}$, for any $\alpha$. We paraphrase this by saying that the universe of GRIST is symmetric under "translations" from $\mathbb{S}$ to $\mathbb{S}_{\alpha}$; every set $\alpha$ has its own "picture of the internal cosmos", given by $\sqsubseteq_{\alpha}$, but these pictures are invariant under the choice of $\alpha$. However, one exception remains: Separation and Replacement schemata of ZFC apply in GRIST to $\in$-formulas only. There seems to be no foundational justification for this. The $\epsilon$ - $\sqsubseteq$-formulas are just as definite (ie, true or false, for given values of their free variables) as $\in$-formulas, and should thus define sets. But if collections like $\Omega:=\{n \in \omega: 0 \sqsubset n\}$ are sets, they can only be external to the universe of GRIST. We face again the question of extending GRIST to a theory that also allows external sets.

The analogous problem for BST is discussed extensively in Kanovei and Reeken's monograph [22]; see also [14]. Here we briefly consider how the solutions that work for BST fare for GRIST. It has to be noted up front that the axiom of Regularity has to fail in any reasonable universe containing external sets (consider the set $\Omega$ above), so the best we can hope for is to satisfy $\mathbf{Z F C}^{-}$(ZFC without Regularity, but with Replacement strengthened to Collection).

The theory HST is an extension of BST to a theory of external sets "with the same ordinals" (ie, the ordinals of the universe of all sets are isomorphic to the ordinals of the standard universe) satisfying all of $\mathbf{Z F C}^{-}$except Power Set and Choice. In particular, Collection holds in HST for all $\in$-st-formulas. It follows from Proposition 12.33 that 
an extension of GRIST to an HST-like theory, where Collection would hold for all $\in$ - -formulas, is impossible.

Another way to extend BST to a theory of external sets "with the same ordinals" is NST $\left(+\mathbb{W F}^{\text {feas }}=\mathbb{W}\right.$ ) $)$, where all axioms of $\mathbf{Z F C}^{-}$except Collection hold. It is easy to see that GRIST can be consistently extended to an NST-like theory, where in particular the schema of Separation holds for all $\in-\sqsubseteq$-formulas. Such theory would seem to provide all the external sets needed in practice of nonstandard analysis. It allows work with monads and galaxies, and the construction of Loeb measures [12] and nonstandard hulls.

The third possibility is to imitate the approach taken by Kawaï [24, 25] in order to extend the universe of IST by external sets (his theory is called KST in [22]). The universe of KST satisfies all of $\mathbf{Z F C}{ }^{-}$; however, it does not "have the same ordinals" as the universe $\mathbb{S}$ of standard sets, which is itself an external set in KST. KST also enables the practice of nonstandard analysis to the full extent. It is not difficult to modify KST to make its internal universe be that of GRIST, rather than IST.

Both of the last two possibilities seem to answer the practical needs of those who might like to be able to combine internal methods of GRIST with the external methods of traditional nonstandard analysis in one theoretical framework. From the foundational viewpoint, however, they appear ad-hoc. Principled comprehensive set-theoretic foundations for nonstandard methods seem to call for an extension of the "Full Relativization" idea from internal sets to all sets. This view was strongly advocated by Ballard in [4]. In the present context, it would mean that every set $\alpha$ has its own "picture of the cosmos", including its own standard universe $\mathbb{S}_{\alpha}$, internal universe $\mathbb{I}_{\alpha}$ with its $\sqsubseteq_{\alpha}$, and a corresponding KST-like external universe $\mathbb{E}_{\alpha}$, and these pictures are invariant under the choice of $\alpha$. An analogous "three-universes" paradigm occurs in mainstream set theory, in the study of both elementary embeddings and Boolean-valued models. Some promising steps towards a formulation of a general theory that would cover all of these situations have been taken, in particular by Ballard in an unpublished paper [5]. However, development of a satisfactory system for full relative set theory is quite a challenge, on both technical and philosophical grounds. We hope to report on the progress towards such a system in a future publication.

\section{References}

[1] P V Andreev, The notion of relative standardness in axiomatic systems of nonstandard analysis, thesis, Dept. Math. Mech., State University of N I Lobachevskiy in Nizhniy Novgorod, 2002, 96 pages (in Russian). 
[2] PV Andreev and EI Gordon, An axiomatics for nonstandard set theory, based on von Neumann-Bernays-Gödel theory, J. Symbolic Logic 66 (2001), 1321-1341; doi: $10.2307 / 2695109$

[3] P V Andreev and K Hrbacek, Standard sets in nonstandard set theory, J. Symbolic Logic 69 (2004), 165-182; doi:10.2178/jsl/1080938835

[4] D Ballard, Foundational Aspects of "Non" standard Mathematics, Contemporary Mathematics, vol. 176, American Mathematical Society, Providence, RI, 1994.

[5] D Ballard, A set theoretic cosmology for mathematics, unpublished paper, January 2002, 22 pages.

[6] C C Chang and H J Keisler, Model Theory, 3rd Edition, North-Holland Publ. Co., 1990, $\mathrm{xii}+550$ pages.

[7] W W Comfort and S Negrepontis, The Theory of Ultrafilters, Grundl. math. Wiss., Vol. 211, Springer-Verlag, New York - Heidelberg, 1974, x + 482 pages.

[8] M A Dickmann, Large Infinitary Languages, Model Theory, Studies in Logic and Found. Math., Vol. 83, North-Holland/American Elsevier, Amsterdam and Oxford/New York, $\mathrm{xv}+464$ pages.

[9] H Gaifman, Elementary embeddings of models of set theory, in Axiomatic Set Theory, Proceedings of Symposia in Pure Math 13, Part II, ed. by T Jech, Amer. Math. Soc., Providence, RI, 1974, 33-101.

[10] E I Gordon, Relatively nonstandard elements in the theory of internal sets of E. Nelson, Siberian Math. J. 30 (1989), 89-95 (in Russian).

[11] K Hrbacek, Axiomatic foundations for nonstandard analysis, Fund. Math. 98 (1978), 1-19; abstract in J. Symbolic Logic 41 (1976), 285.

[12] K Hrbacek, Nonstandard set theory, Amer. Math. Monthly 86 (1979), 1-19; doi:10.2307/2321294

[13] K Hrbacek, Internally iterated ultrapowers, in Nonstandard Models of Arithmetic and Set Theory, ed. by A Enayat and R Kossak, Contemporary Math. 361, American Mathematical Society, Providence, RI, 2004, 87-120.

[14] K Hrbacek, Nonstandard objects in set theory, in Nonstandard Methods and Applications in Mathematics, ed. by N J Cutland, M Di Nasso and D A Ross, Lecture Notes in Logic 25, Association for Symbolic Logic, 2006, 80-120.

[15] K Hrbacek, Some remarks on nonstandard theory of classes, Fundamentalnaya i Prikladnaya Matematika, 11, 5 (2005), 233-255 (in Russian). [English transl. Journal of Math. Sciences, 146, 1 (2007), 5608-5621]; doi:10.1007/s10958-007-0374-0

[16] K Hrbacek, Stratified analysis?, in The Strength of Nonstandard Analysis, ed. by I van den Berg and V Neves, Springer Wien New York, 2007, 47-63.

[17] K Hrbacek, O Lessmann and R O’Donovan, Analysis with ultrasmall numbers, 16 pages, March 17, 2009. 
[18] K Hrbacek, O Lessmann and R O'Donovan, Analysis using Relative Infinitesimals, 267 pages, March 19, 2008.

[19] T Jech, Set Theory, Academic Press, New York 1978, xi + 621 pages.

[20] V Kanovei, Undecidable hypotheses in Edward Nelson's Internal Set Theory, Russian Mathematical Surveys 46 (1991), 1-54; doi:10.1070/RM1991v046n06ABEH002870

[21] V Kanovei and M Reeken, Internal approach to external sets and universes, Studia Logica, Part I, 55 (1995), 227-235; Part II, 55 (1995), 347-376; Part III, 56 (1996), 293-322; doi:10.1007/BF01061236; 10.1007/BF01057803; 10.1007/BF00372770

[22] V Kanovei and M Reeken, Nonstandard Analysis: Axiomatically, Springer-Verlag Berlin Heidelberg New York, 2004, xvi +408 pages.

[23] V Kanovei and S Shelah, A definable nonstandard model of the reals, J. Symbolic Logic 69 (2004), 160-164; doi:10.2178/js1/1080938834

[24] T Kawaï, Axiom systems of nonstandard set theory, in G Müller at al., (Eds.), Logic Symposia, Hakone 1979, 1980, Lecture Notes in Math. Vol. 891, Springer, Berlin, 1981, 57-65.

[25] T Kawaï, Nonstandard analysis by axiomatic method, in C T Chong, M J Wicks, (Eds.), Southeast Asean Conference on Logic, Proc. Singapore 1981, Studies in Logic and the Foundations of Math., Vol. 111, North-Holland, Amsterdam, 1983, 55-76.

[26] K Kunen, Some applications of iterated ultrapowers in set theory, Ann. Math. Logic 1 (1970), 179-227; doi:10.1016/0003-4843(70)90013-6

[27] E Nelson, Internal set theory: a new approach to Nonstandard Analysis, Bull. Amer. Math. Soc. 83 (1977), 1165-1198; doi:10.1090/S0002-9904-1977-14398-X

[28] Y Péraire, Théorie relative des ensembles intérnes, Osaka J. Math. 29 (1992), 267-297.

[29] Y Péraire, Formules absolues dans la théorie relative des ensembles intérnes, Rivista di Matematica Pura ed Applicata 19 (1996), 27-56.

[30] Y Péraire, Infinitesimal approach of almost-automorphic functions, Ann. Pure Appl. Logic 63 (1993), 283-297; doi:10.1016/0168-0072(93)90152-4

[31] A Robinson and E Zakon, A set-theoretical characterization of enlargements, in W A J Luxemburg, ed., Applications of Model Theory to Algebra, Analysis and Probability, Holt, Rinehart and Winston 1969, pp. 109-122.

[32] D Scott, Measurable cardinals and constructible sets, Bull. Acad. Polon. Sci. 9 (1961), $521-524$.

[33] J R Shoenfield, Mathematical Logic, Addison-Wesley Publ. Co., Inc., 1967, viii + 344 pages.

[34] P Vopěnka, Construction of models of set theory by the method of ultraproducts, Zeitschrift für Math. Logik 8 (1962), 293-304 (in Russian).

The City College of New York

khrbacek@sci.ccny. cuny. edu

Received: 21 November 2008 Revised: 25 May 2009 